\title{
Photochemical Route to the Synthesis of Thiolane 1-Oxides
}

\author{
Jeffrey D. Winkler* and Esther C. Y. Lee \\ Department of Chemistry, University of Pennsylvania, Philadelphia, PA 19104 \\ winkler@sas.upenn.edu
}

\section{Supplementary Information}

Table of Contents

Experimental Section

S5-S19

${ }^{1} \mathrm{H}$ NMR Spectrum of $\mathbf{1}$

S20

${ }^{13} \mathrm{C}$ NMR Spectrum of 1

S21

IR Spectrum of 1

$\mathrm{S} 22$

${ }^{1} \mathrm{H}$ NMR Spectrum of $\mathbf{3}$

S23

${ }^{13} \mathrm{C}$ NMR Spectrum of $\mathbf{3}$

S24

IR Spectrum of $\mathbf{3}$

$\mathrm{S} 25$

${ }^{1}$ H NMR Spectrum of 2,2-Dimethyl-6-vinyl-[1,3]dioxen-4-one

S26

${ }^{13} \mathrm{C}$ NMR Spectrum of 2,2-Dimethyl-6-vinyl-[1,3]dioxen-4-one

S27

IR Spectrum of 2,2-Dimethyl-6-vinyl-[1,3]dioxen-4-one S28

${ }^{1} \mathrm{H}$ NMR Spectrum of $\mathbf{4 a}$

S29

${ }^{13} \mathrm{C}$ NMR Spectrum of $\mathbf{4 a}$

S30

IR Spectrum of $\mathbf{4 a}$

S31

${ }^{1} \mathrm{H}$ NMR Spectrum of $\mathbf{4 b}$

S32

${ }^{13} \mathrm{C}$ NMR Spectrum of $\mathbf{4 b}$

S33

IR Spectrum of $\mathbf{4 b}$

S34

${ }^{1} \mathrm{H}$ NMR Spectrum of $\mathbf{4 c}$

S35

${ }^{13} \mathrm{C}$ NMR Spectrum of $\mathbf{4 c}$

S36

IR Spectrum of $\mathbf{4 c}$

S37

${ }^{1} \mathrm{H}$ NMR Spectrum of $\mathbf{5 a}$

S38

${ }^{13} \mathrm{C}$ NMR Spectrum of $\mathbf{5 a}$

S39 
IR Spectrum of 5a $\quad$ S40

${ }^{1} \mathrm{H}$ NMR Spectrum of $\mathbf{5 b} \quad$ S41

${ }^{13} \mathrm{C}$ NMR Spectrum of $\mathbf{5 b} \quad \mathrm{S} 42$

IR Spectrum of $\mathbf{5 b} \quad \mathrm{S} 43$

${ }^{1} \mathrm{H}$ NMR Spectrum of $\mathbf{5 b}, \quad$ S44

${ }^{13}$ C NMR Spectrum of $\mathbf{5 b}, \quad$ S45

IR Spectrum of $\mathbf{5 b}, \quad$ S46

${ }^{1} \mathrm{H}$ NMR Spectrum of $\mathbf{5 c} \quad$ S47

${ }^{13} \mathrm{C}$ NMR Spectrum of $\mathbf{5 c} \quad \mathrm{S} 48$

IR Spectrum of $\mathbf{5 c} \quad$ S49

${ }^{1} \mathrm{H}$ NMR of 3-[2-(4-Methoxy-phenylsulfanyl)-ethyl]-cyclohex-2-enone S50

${ }^{13}$ C NMR of 3-[2-(4-Methoxy-phenylsulfanyl)-ethyl]-cyclohex-2-enone S51

IR of 3-[2-(4-Methoxy-phenylsulfanyl)-ethyl]-cyclohex-2-enone S52

${ }^{1} \mathrm{H}$ NMR Spectrum of 9

${ }^{13} \mathrm{C}$ NMR Spectrum of $9 \quad \mathrm{~S} 54$

IR Spectrum of 9

${ }^{1} \mathrm{H}$ NMR Spectrum of $\mathbf{1 0}\left(\mathbf{R}_{\mathrm{f}}=\mathbf{0 . 3}\right.$ in $\mathbf{9 0 \%}$ ethyl acetate/hexane $) \quad$ S56

${ }^{13} \mathrm{C}$ NMR Spectrum of $\mathbf{1 0}\left(\mathbf{R}_{\mathrm{f}}=\mathbf{0 . 3}\right.$ in $\mathbf{9 0 \%}$ ethyl acetate/hexane $) \quad$ S57

IR Spectrum of $\mathbf{1 0}\left(\mathbf{R}_{\mathrm{f}}=\mathbf{0 . 3}\right.$ in $\mathbf{9 0 \%}$ ethyl acetate/hexane $) \quad$ S58

${ }^{1} \mathrm{H}$ NMR Spectrum of $\mathbf{1 0}\left(\mathbf{R}_{\mathrm{f}}=\mathbf{0 . 2}\right.$ in $\mathbf{9 0 \%}$ ethyl acetate/hexane $) \quad$ S59

${ }^{13} \mathrm{C}$ NMR Spectrum of $\mathbf{1 0}\left(\mathbf{R}_{\mathrm{f}}=\mathbf{0 . 2}\right.$ in $\mathbf{9 0 \%}$ ethyl acetate/hexane $) \quad$ S60

IR Spectrum of $\mathbf{1 0}\left(\mathbf{R}_{\mathrm{f}}=\mathbf{0 . 2}\right.$ in $\mathbf{9 0 \%}$ ethyl acetate/hexane $) \quad$ S61

${ }^{1} \mathrm{H}$ NMR of 3-(2-Phenylsulfanyl-ethyl)-cyclohex-2-enone S62

${ }^{13} \mathrm{C}$ NMR of 3-(2-Phenylsulfanyl-ethyl)-cyclohex-2-enone S63

IR of 3-(2-Phenylsulfanyl-ethyl)-cyclohex-2-enone S64

${ }^{1} \mathrm{H}$ NMR Spectrum of $\mathbf{1 1} \quad$ S65

${ }^{13}$ C NMR Spectrum of $\mathbf{1 1}$ S66

$\begin{array}{ll}\text { IR Spectrum of } \mathbf{1 1} & \text { S67 }\end{array}$

${ }^{1} \mathrm{H}$ NMR Spectrum of $\mathbf{1 2}\left(\mathbf{R}_{\mathrm{f}}=\mathbf{= 0 . 2}\right.$ in $\mathbf{8 0 \%}$ ethyl acetate/hexane $) \quad$ S68

${ }^{13} \mathrm{C}$ NMR Spectrum of $\mathbf{1 2}\left(\mathbf{R}_{\mathrm{f}}=\mathbf{0 . 2}\right.$ in $\mathbf{8 0 \%}$ ethyl acetate/hexane $) \quad \mathrm{S} 69$

IR Spectrum of $12\left(\mathbf{R}_{\mathrm{f}}=\mathbf{0 . 2}\right.$ in $80 \%$ ethyl acetate/hexane $) \quad S 70$ 
${ }^{1} \mathrm{H}$ NMR Spectrum of $\mathbf{1 2}\left(\mathbf{R}_{\mathrm{f}}=\mathbf{= 0 . 1}\right.$ in $\mathbf{8 0 \%}$ ethyl acetate/hexane $) \quad$ S71

${ }^{13}$ C NMR Spectrum of $\mathbf{1 2}\left(\mathbf{R}_{\mathbf{f}}=\mathbf{0 . 1}\right.$ in $\mathbf{8 0 \%}$ ethyl acetate/hexane $) \quad$ S72

IR Spectrum of $12\left(\mathbf{R}_{\mathbf{f}}=\mathbf{0 . 1}\right.$ in $\mathbf{8 0 \%}$ ethyl acetate/hexane $) \quad$ S73

${ }^{1} \mathrm{H}$ NMR of 3-[2-(2-Hydroxy-phenylsulfanyl)-ethyl]-cyclohex-2-enone $\mathrm{S} 74$

${ }^{13} \mathrm{C}$ NMR of 3-[2-(2-Hydroxy-phenylsulfanyl)-ethyl]-cyclohex-2-enone S75

IR of 3-[2-(2-Hydroxy-phenylsulfanyl)-ethyl]-cyclohex-2-enone $\quad$ S76

$\begin{array}{ll}{ }^{1} \mathrm{H} \text { NMR Spectrum of } \mathbf{1 3} & \text { S77 }\end{array}$

${ }^{13} \mathrm{C}$ NMR Spectrum of $\mathbf{1 3} \quad$ S78

$\begin{array}{ll}\text { IR Spectrum of } \mathbf{1 3} & \text { S79 }\end{array}$

${ }^{1} \mathrm{H}$ NMR Spectrum of $\mathbf{1 4} \quad \mathrm{S} 80$

${ }^{13} \mathrm{C}$ NMR Spectrum of $\mathbf{1 4} \quad$ S81

IR Spectrum of $\mathbf{1 4} \quad$ S82

${ }^{1} \mathrm{H}$ NMR Spectrum of $\mathbf{1 5} \quad$ S83

${ }^{13} \mathrm{C}$ NMR Spectrum of $\mathbf{1 5} \quad$ S84

IR Spectrum of 15

${ }^{1} \mathrm{H}$ NMR Spectrum of $\mathbf{1 6} \quad$ S86

$\begin{array}{ll}{ }^{13} \mathrm{C} \text { NMR Spectrum of } \mathbf{1 6} & \text { S87 }\end{array}$

IR Spectrum of $16 \quad$ S88

${ }^{1} \mathrm{H}$ NMR of 3-[2-(2-Amino-phenylsulfanyl)-ethyl]-cyclohex-2-enone $\quad$ S89

${ }^{13} \mathrm{C}$ NMR of 3-[2-(2-Amino-phenylsulfanyl)-ethyl]-cyclohex-2-enone $\quad \mathrm{S} 90$

IR of 3-[2-(2-Amino-phenylsulfanyl)-ethyl]-cyclohex-2-enone $\quad$ S91

$\begin{array}{ll}{ }^{1} \mathrm{H} \text { NMR Spectrum of } \mathbf{1 7} & \mathrm{S} 92\end{array}$

${ }^{13}$ C NMR Spectrum of $\mathbf{1 7} \quad$ S93

$\begin{array}{ll}\text { IR Spectrum of } \mathbf{1 7} & \text { S94 }\end{array}$

$\begin{array}{ll}{ }^{1} \mathrm{H} \text { NMR Spectrum of } \mathbf{1 8} & \mathrm{S} 95\end{array}$

${ }^{13}$ C NMR Spectrum of $\mathbf{1 8} \quad$ S96

$\begin{array}{ll}\text { IR Spectrum of } \mathbf{1 8} & \text { S97 }\end{array}$

$\begin{array}{ll}{ }^{1} \mathrm{H} \text { NMR Spectrum of } \mathbf{1 9} & \mathrm{S} 98\end{array}$

$\begin{array}{ll}{ }^{13} \mathrm{C} \text { NMR Spectrum of } \mathbf{1 9} & \text { S99 }\end{array}$

IR Spectrum of 19

$\begin{array}{ll}{ }^{1} H \text { NMR Spectrum of } 20 & \text { S101 }\end{array}$ 
$\begin{array}{lr}{ }^{13} \mathrm{C} \text { NMR Spectrum of } \mathbf{2 0} & \text { S102 }\end{array}$

$\begin{array}{lr}\text { IR Spectrum of } \mathbf{2 0} & \text { S103 }\end{array}$

$\begin{array}{lr}{ }^{1} \mathrm{H} \text { NMR Spectrum of } \mathbf{2 1} & \text { S104 }\end{array}$

$\begin{array}{ll}{ }^{13} \mathrm{C} \text { NMR Spectrum of } 21 & \text { S105 }\end{array}$

$\begin{array}{ll}\text { IR Spectrum of } 21 & \text { S106 }\end{array}$

$\begin{array}{ll}{ }^{1} \mathrm{H} \text { NMR Spectrum of } \mathbf{2 2} & \text { S107 }\end{array}$

$\begin{array}{lr}{ }^{13} \mathrm{C} \text { NMR Spectrum of } 22 & \text { S108 }\end{array}$

$\begin{array}{lr}\text { IR Spectrum of } 22 & \text { S109 }\end{array}$

$\begin{array}{lr}{ }^{1} \mathrm{H} \text { NMR Spectrum of 22'} & \text { S110 }\end{array}$

$\begin{array}{ll}{ }^{13} \mathrm{C} \text { NMR Spectrum of 22'} & \text { S111 }\end{array}$

$\begin{array}{lr}\text { IR Spectrum of 22' } & \text { S112 }\end{array}$

$\begin{array}{lr}\text { X-ray Structure Determination of } \mathbf{5 b} & \text { S113-120 }\end{array}$

$\begin{array}{lr}\text { X-ray Structure Determination of 5b' } & \text { S121-127 }\end{array}$ 


\title{
Supporting Information
}

\section{Photochemical Route to the Synthesis of Thiolane 1-Oxides}

\author{
Jeffrey D. Winkler* and Esther C. Y. Lee \\ Department of Chemistry, University of Pennsylvania, Philadelphia, PA 19104
}

\section{EXPERIMENTAL DETAILS}

\section{General Methods}

Solvents used for extraction and purification were HPLC grade from Fisher. Unless otherwise indicated, all reactions were run under an inert atmosphere of Argon. Anhyrous tetrahydrofuran, methylene chloride, benzene, and acetonitrile were obtained via passage through an activated alumina column ${ }^{1}$. Acetonitrile used for photoreactions was taken from the Grubbs system and pre-irradiated for 4 hours with a 450 Watt Hanovia mercury lamp at $0{ }^{\circ} \mathrm{C}$ followed by distillation. Acetone used for photoreactions was freshly distilled from HPLC grade Fisher each time prior to use. A triple-jacketed Pyrex ${ }^{\mathrm{TM}}$ photoreactor was used for photochemical reactions. Commercial reagents were used as received. Deuterated solvents were obtained from Cambridge Isotope Labs. Merck pre-coated silica gel plates $\left(250 \mu \mathrm{M}, 60 \mathrm{~F}_{254}\right)$ were used for analytical TLC. Spots were visualized using $254 \mathrm{~nm}$ ultraviolet light, with either anisaldehyde or potassium permangenate stains as visualizing agents. Chromatographic purifications were performed on Sorbent Technologies silica gel (particle size 32-63 microns). ${ }^{1} \mathrm{H}$ and ${ }^{13} \mathrm{C}$ NMR spectra were recorded on $500 \mathrm{MHz}$ and $125 \mathrm{MHz}$, respectively, in $\mathrm{CDCl}_{3}$ on a Bruker AM-500 or DRX-500 spectrometer. Chemical shifts are reported relative to internal chloroform $\left(\delta 7.26\right.$ for ${ }^{1} \mathrm{H}, \delta 77.0$ for $\left.{ }^{13} \mathrm{C}\right)$. Infrared spectra were recorded on a $\mathrm{NaCl}$ plate using a Perkin-Elmer 1600 series Fourier transform spectrometer. High resolution mass spectra were obtained by Dr. Rakesh Kohli at the University of Pennsylvania Mass Spectrometry Service Center on an Autospec high resolution doublefocusing electrospray ionization/chemical ionization spectrometer with either DEC 11/73 or OPUS software data system. Single-crystal X-ray diffraction structure determination was performed by Dr. Pat Caroll at the University of Pennsylvania. Melting points were obtained on a Thomas Hoover capillary melting point apparatus and are uncorrected.

\footnotetext{
${ }^{1}$ Pangborn, A. B.; Giardello, M. A.; Grubbs, R. H.; Rosen, R. K.; Timmers, F. J. Organometallics 1996, 15, 1518.
} 

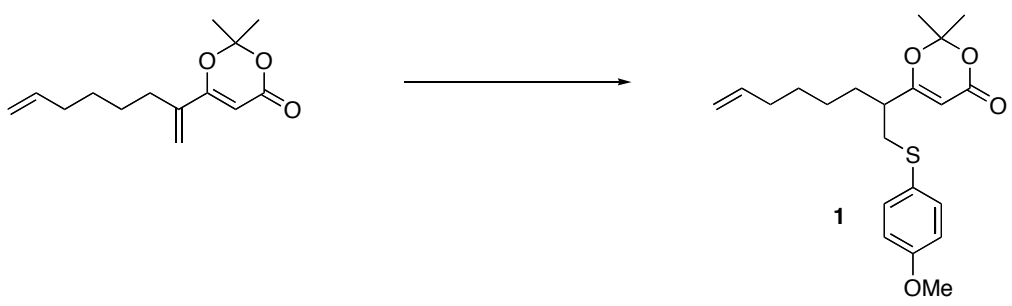

6-[1-(4-Methoxy-phenylsulfanylmethyl)-hept-6-enyl]-2,2-dimethyl-[1,3]dioxen-4-one 1. In a flame dried flask was added 2,2-Dimethyl-6-(1-methylene-hept-6-enyl)[1,3]dioxen-4-one $(81 \mathrm{mg}, 0.34 \mathrm{mmol})$ and dry methylene choride $(6.8 \mathrm{~mL}, 0.05 \mathrm{M})$ at 25 ${ }^{\circ} \mathrm{C}$. Trietheylamine $(0.133 \mathrm{~mL}, 0.95 \mathrm{mmol})$ was next added dropwise followed by 4methoxybenzene thiol $(0.048 \mathrm{~mL}, 0.39 \mathrm{mmol})$. The reaction was stirred at $25^{\circ} \mathrm{C}$ for $18 \mathrm{~h}$. The reaction was diluted with methylene chloride/pH 7 buffer and extracted with methylene chloride. The combined organics were washed with $\mathrm{pH} 7$ buffer, dried, filtered, and concentrated. Purification by silica gel chromatography (10\%-50\% ether/hexane) afforded 6-[1-(4-Methoxy-phenylsulfanylmethyl)-hept-6-enyl]-2,2dimethyl-[1,3]dioxen-4-one 1 (108 mg, 84\%) as an oil: ${ }^{1} \mathrm{H}$ NMR (500 MHz) $\delta 7.32$ (d, $J$ $=8.7 \mathrm{~Hz}, 2 \mathrm{H}), 6.84(\mathrm{~d}, J=8.7 \mathrm{~Hz}, 2 \mathrm{H}), 5.74(\mathrm{dddd}, J=16.9,10.2,6.7,6.7 \mathrm{~Hz}, 1 \mathrm{H}), 5.20$ (s, 1H), 4.97 (dd, $J=17.1,1.7 \mathrm{~Hz}, 1 \mathrm{H}), 4.93(\mathrm{dd}, J=10.2,0.9 \mathrm{~Hz}, 1 \mathrm{H}), 3.79(\mathrm{~s}, 3 \mathrm{H}), 2.92$ (dd, $J=13.3,8.7 \mathrm{~Hz}, 1 \mathrm{H}), 2.87$ (dd, $J=13.4,5.8 \mathrm{~Hz}, 1 \mathrm{H}), 2.31$ (dddd (dt), $J=7.2,7.2$, 7.2, 7.2 Hz, 1H), 2.0 (ddd, (dt), $J=6.9,6.9,6.9 \mathrm{~Hz}, 2 \mathrm{H}), 1.68(\mathrm{~s}, 3 \mathrm{H}), 1.66(\mathrm{~s}, 3 \mathrm{H}), 1.54$ (ddd, (dt), $J=7.8,7.8,7.8 \mathrm{~Hz}, 2 \mathrm{H}), 1.35-1.24(\mathrm{~m}, 4 \mathrm{H}) ;{ }^{13} \mathrm{C}$ NMR $(125 \mathrm{MHz}) \delta 171.8$, 161.0, 159.3, 138.4, 133.8, 125.3, 114.8, 114.6, 106.5, 94.9, 55.3, 44.2, 37.9, 33.3, 31.3, 28.5, 26.4, 25.4, 24.8; FTIR (thin film) 2996, 2933, 2856, 1732, 1632, 1592, 1570, 1494, $1381,1376,1272,1246,1202,1180,1027,901,828 \mathrm{~cm}^{-1}$. Exact mass calculated for $\mathrm{C}_{21} \mathrm{H}_{28} \mathrm{O}_{4} \mathrm{~S}+\mathrm{Na}^{+} 399.1408$, found 399.1598.
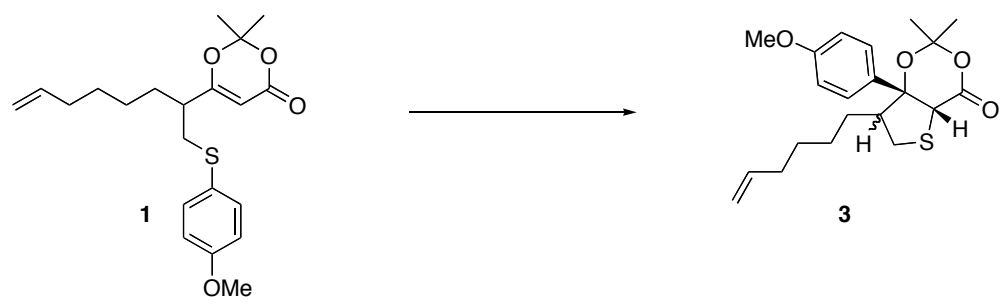

Thiolane Dioxanone 3. In an oven-dried $250 \mathrm{~mL}$ photoreactor with Pyrex $^{\mathrm{TM}}$ insert was added 6-[1-(4-Methoxy-phenylsulfanylmethyl)-hept-6-enyl]-2,2-dimethyl-[1,3]dioxen-4one $1(100 \mathrm{mg}, 0.27 \mathrm{mmol})$ with a 9:1 mixture of pre-irradiated and distilled acetonitrile and freshly distilled acetone $(120.0 \mathrm{~mL} / 13.0 \mathrm{~mL}, 2.0 \mathrm{mM})$. The mixture was purged at 0 ${ }^{\circ} \mathrm{C}$ under argon for $1 \mathrm{~h}$. The reaction was irradiated for $50 \mathrm{~min}$ and concentrated. Purification by silica gel chromatography (50\% methylene chloride/hexane w $0.2 \%$ Ether) afforded thiolane dioxanone $3(26 \mathrm{mg}, 26 \%)$ as an oil: ${ }^{1} \mathrm{H}$ NMR (500 MHz) $\delta 7.46$ $(\mathrm{d}, J=8.8 \mathrm{~Hz}, 2 \mathrm{H}), 7.43(\mathrm{~d}, J=8.8 \mathrm{~Hz}, 2 \mathrm{H}), 6.90(\mathrm{~d}, J=8.8 \mathrm{~Hz}, 4 \mathrm{H}), 5.73(\mathrm{~m}, 2 \mathrm{H}), 4.93$ $(\mathrm{m}, 4 \mathrm{H}), 4.39(\mathrm{~s}, 1 \mathrm{H}), 4.32(\mathrm{~s}, 1 \mathrm{H}), 3.82(\mathrm{~s}, 3 \mathrm{H}), 3.81(\mathrm{~s}, 3 \mathrm{H}), 3.09$ (dd, $J=9.9,5.3 \mathrm{~Hz}$, $1 \mathrm{H}), 2.76(\mathrm{t}, J=4.8 \mathrm{~Hz}, 2 \mathrm{H}), 2.44(\mathrm{~m}, 1 \mathrm{H}), 2.39(\mathrm{t}, J=9.9 \mathrm{~Hz}, 1 \mathrm{H}), 2.15(\mathrm{~m}, 1 \mathrm{H}), 2.00$ $(\mathrm{m}, 2 \mathrm{H}), 1.96(\mathrm{dd}, J=13.1,6.5 \mathrm{~Hz}, 2 \mathrm{H}), 1.70(\mathrm{~s}, 3 \mathrm{H}), 1.66(\mathrm{~s}, 3 \mathrm{H}), 1.35(\mathrm{~m}, 3 \mathrm{H}), 1.30(\mathrm{~s}$, 
$3 \mathrm{H}), 1.24(\mathrm{~m}, 7 \mathrm{H}), 1.17(\mathrm{~s}, 3 \mathrm{H}), 0.87(\mathrm{~m}, 1 \mathrm{H}), 0.72(\mathrm{~m}, 1 \mathrm{H}) ;{ }^{13} \mathrm{C} \mathrm{NMR}(125 \mathrm{MHz})$

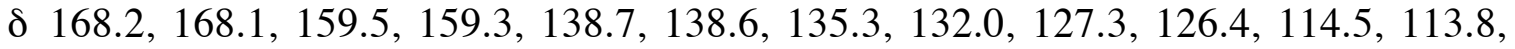
$113.7,106.9,106.8,88.5,88.1,57.5,55.7,55.2,50.2,48.6,33.6,33.4,32.5,32.2,29.9$, 29.4, 28.8, 28.7, 28.1, 27.6, 27.5, 27.3, 26.9, 26.8; FTIR (thin film) 2998, 2933, 2857, $1747,1610,1512,1463,1442,1387,1298,1253,1182,1112,1035,994,835 \mathrm{~cm}^{-1}$. Exact mass calculated for $\mathrm{C}_{21} \mathrm{H}_{28} \mathrm{O}_{4} \mathrm{~S}+\mathrm{H}^{+}$(ES) 377.1786, found 377.1772.
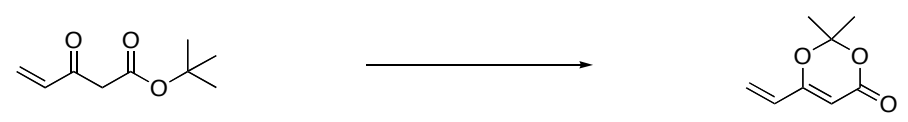

2,2-Dimethyl-6-vinyl-[1,3]dioxen-4-one. To a flask containing, unsaturated 3-oxo-pent4-enoic acid tert-butyl ester $(7.0 \mathrm{~g}, 41.2 \mathrm{mmol})$ was added acetone $(343.0 \mathrm{~mL}, 0.12 \mathrm{M})$ and the resulting solution was cooled to $-78^{\circ} \mathrm{C}$. Trifluoroacetic anhydride $(69.0 \mathrm{~mL}, 0.6$ M) followed by trifluoroacetic acid $(231.0 \mathrm{~mL}, 0.18 \mathrm{M})$ and acetic anhydride $(56.0 \mathrm{~mL}$, $0.74 \mathrm{M}$ ) were added dropwise. The reaction was gradually warmed to $25{ }^{\circ} \mathrm{C}$ and stirred for $18 \mathrm{~h}$. The reaction was poured into a Erlenmeyer containing cold satuarated $\mathrm{NaHCO}_{3}$ and extracted with ethyl acetate $(5 x)$. The combined organic layers were dried, filtered, and concentrated. Purification by silica gel chromatography (15\% ethyl acetate/hexane) afforded 2,2-Dimethyl-6-vinyl-[1,3]dioxen-4-one (3.2 g, 50\%) as an oil: ${ }^{1} \mathrm{H}$ NMR (500 MHz) $\delta 6.20(\mathrm{dd}, J=17.2,10.7 \mathrm{~Hz}, 1 \mathrm{H}), 6.00(\mathrm{dd}, J=17.2,0.7 \mathrm{~Hz}, 1 \mathrm{H}), 5.59(\mathrm{dd}, J=$ 10.7, 0.5 Hz, 1H), $5.34(\mathrm{~s}, 1 \mathrm{H}), 1.69(\mathrm{~s}, 6 \mathrm{H}) ;{ }^{13} \mathrm{C}$ NMR $(125 \mathrm{MHz}) \delta 162.8,161.6,129.3$, 123.8, 106.5, 95.2, 25.0; FTIR (thin film) 1731, 1645, 1582, 1391, 1274, 1252, 1205, $1099,1045,999,903,816,668 \mathrm{~cm}^{-1}$. Exact mass calculated for $\mathrm{C}_{8} \mathrm{H}_{10} \mathrm{O}_{3}{ }^{+}$(CI) 155.0708, found 155.0712.
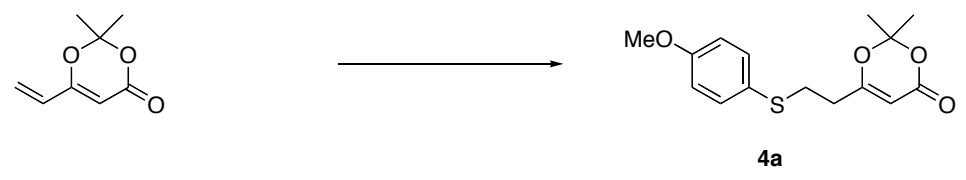

6-[2-(4-Methoxy-phenylsulfanyl)-ethyl]-2,2-dimethyl-[1,3]dioxen-4-one 4a. In a flame dried flask, was added 2,2-Dimethyl-6-vinyl-[1,3]dioxen-4-one (200 mg, 1.3 $\mathrm{mmol})$ and dry methylene chloride $(26.0 \mathrm{~mL}, 0.05 \mathrm{M})$. Triethylamine $(0.545 \mathrm{~mL}, 3.9$ $\mathrm{mmol})$ was next added followed by 4-methoxybenzene thiol $(0.221 \mathrm{~mL}, 1.8 \mathrm{mmol})$. The reaction was stirred overnight at $25{ }^{\circ} \mathrm{C}$. The reaction was diluted with methylene chloride/pH 7 buffer and extracted with methylene chloride. The combined organics were washed with $\mathrm{pH} 7$ buffer, dried, filtered, and concentrated. Purification by silica gel chromatography (10\% ethyl acetate/hexane) afforded 6-[2-(4-Methoxy-phenylsulfanyl)ethyl]-2,2-dimethyl-[1,3]dioxen-4-one $\mathbf{4 a}(302 \mathrm{mg}, 79 \%)$ as an oil: ${ }^{1} \mathrm{H}$ NMR $(500 \mathrm{MHz})$ $\delta 7.36(\mathrm{~d}, J=8.7 \mathrm{~Hz}, 2 \mathrm{H}), 6.86(\mathrm{~d}, J=8.7 \mathrm{~Hz}, 2 \mathrm{H}), 5.23(\mathrm{~s}, 1 \mathrm{H}), 3.80(\mathrm{~s}, 3 \mathrm{H}), 2.96(\mathrm{t}, J=$ $7.2 \mathrm{~Hz}, 2 \mathrm{H}), 2.45$ (t, $J=7.2 \mathrm{~Hz}, 2 \mathrm{H}), 1.67(\mathrm{~s}, 6 \mathrm{H}) ;{ }^{13} \mathrm{C}$ NMR $(125 \mathrm{MHz}) \delta 169.2,160.9$, 159.5, 134.2, 124.6, 114.8, 106.6, 94.6, 55.3, 33.5, 31.9, 25.1; FTIR (thin film) 2997, 2942, 2835, 1727, 1634, 1591, 1494, 1390, 1374, 1275, 1246, 1203, 1175, 1016, 960, $901,828 \mathrm{~cm}^{-1}$. Exact mass calculated for $\mathrm{C}_{15} \mathrm{H}_{18} \mathrm{O}_{4} \mathrm{~S}+\mathrm{H}^{+}(\mathrm{CI}) 295.1003$, found 295.0993 . 


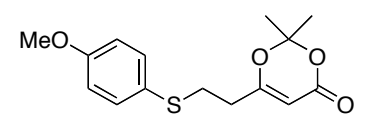

4a

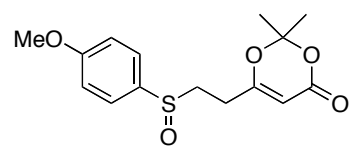

4b

6-[2-(4-Methoxy-benzenesulfinyl)-ethyl]-2,2-dimethyl-[1,3]dioxen-4-one $4 \mathrm{~b}$. To a flask containing 6-[2-(4-Methoxy-phenylsulfanyl)-ethyl]-2,2-dimethyl-[1,3]dioxen-4-one 4a (228 mg, $0.78 \mathrm{mmol})$, was added anhydrous methylene chloride $(39 \mathrm{~mL}, 0.02 \mathrm{M})$ and the soluton was cooled to $-78{ }^{\circ} \mathrm{C}$. A solution of $m$-CPBA $(216 \mathrm{mg}, 1.1 \mathrm{mmol})$ in methylene chloride was added to the cooled solution. The reaction was quenched in 30 min. with saturated $\mathrm{NaHCO}_{3}$ and extracted with methylene chloride $(3 \mathrm{x})$. The combined organic layers were washed with saturated $\mathrm{NaHCO}_{3}$ and dried, filtered, and concentrated. Purification by silica gel chromatography (20\% hexane/ethyl acetate) afforded 6-[2-(4Methoxy-benzenesulfinyl)-ethyl]-2,2-dimethyl-[1,3]dioxen-4-one $\mathbf{4 b}$ (229 $\mathrm{mg}, 95 \%)$ as a foam: ${ }^{1} \mathrm{H}$ NMR $(500 \mathrm{MHz}) \delta 7.50(\mathrm{~d}, J=8.8 \mathrm{~Hz}, 2 \mathrm{H}), 7.0(\mathrm{~d}, J=8.8 \mathrm{~Hz}, 2 \mathrm{H}), 5.20$ (s, $1 \mathrm{H}), 3.81(\mathrm{~s}, 3 \mathrm{H}), 2.98(\mathrm{ddd}, J=13.2,9.6,6.0 \mathrm{~Hz}, 1 \mathrm{H}), 2.85(\mathrm{ddd}, J=13.2,9.5,5.7 \mathrm{~Hz}$, $1 \mathrm{H}), 2.65$ (ddd, $J=15.4,9.4,6.0 \mathrm{~Hz}, 1 \mathrm{H}), 2.39$ (ddd, $J=15.3,9.6,5.6 \mathrm{~Hz}, 1 \mathrm{H}), 1.61$ (s, $6 \mathrm{H}) ;{ }^{13} \mathrm{C}$ NMR $(125 \mathrm{MHz}) \delta 168.2,162.2,160.4,133.2,125.8,114.9,106.7,94.3,55.4$, 51.8, 25.8, 24.9, 24.8; FTIR (thin film) 3460, 3094, 2997, 2943, 2839, 1726, 1634, 1594, 1578, 1496, 1462, 1441, 1391, 1376, 1305, 1275, 1253, 1204, 1180, 1088, 1044, 1017, $903,832 \mathrm{~cm}^{-1}$. Exact mass calculated for $\mathrm{C}_{15} \mathrm{H}_{18} \mathrm{O}_{5} \mathrm{~S}+\mathrm{Na}^{+}$(ES) 333.0800, found 333.0776 .

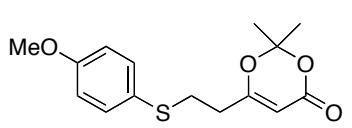

$4 a$

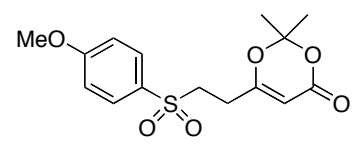

4c

6-[2-(4-Methoxy-benzenesulfonyl)-ethyl]-2,2-dimethyl-[1,3]dioxen-4-one 4c. To a flask containing 6-[2-(4-Methoxy-phenylsulfanyl)-ethyl]-2,2-dimethyl-[1,3]dioxen-4-one 4a (197 mg, $0.67 \mathrm{mmol})$ was added anhydrous methylene chloride $(33.5 \mathrm{~mL}, 0.02 \mathrm{M})$. The resulting solution was cooled to $0^{\circ} \mathrm{C}$. A solution of $m$-CPBA $(510 \mathrm{mg}, 3.0 \mathrm{mmol})$ in methylene chloride was added to the cooled solution. The reaction was quenched in $1 \mathrm{~h}$ with saturated $\mathrm{NaHCO}_{3}$ and extracted with methylene chloride (3x). The combined organic layers were washed with saturated $\mathrm{NaHCO}_{3}$ and dried, filtered, and concentrated. Purification by silica gel chromatography (50\% ethyl acetate/ heaxanes) afforded 6-[2-(4Methoxy-benzenesulfonyl)-ethyl]-2,2-dimethyl-[1,3]dioxen-4-one 4c (218 $\mathrm{mg}, 100 \%)$ as a white solid, mp 81-86 ${ }^{\circ} \mathrm{C}:{ }^{1} \mathrm{H}$ NMR $(500 \mathrm{MHz}) \delta 7.80(\mathrm{~d}, J=8.8 \mathrm{~Hz}, 2 \mathrm{H}), 7.02(\mathrm{~d}, J=$ $8.8 \mathrm{~Hz}, 2 \mathrm{H}), 5.22(\mathrm{~s}, 1 \mathrm{H}), 3.87(\mathrm{~s}, 3 \mathrm{H}), 3.25(\mathrm{t}, J=7.7 \mathrm{~Hz}, 2 \mathrm{H}), 2.64(\mathrm{t}, J=8.0 \mathrm{~Hz}, 2 \mathrm{H})$, $1.61(\mathrm{~s}, 6 \mathrm{H}) ;{ }^{13} \mathrm{C}$ NMR $(125 \mathrm{MHz}) \delta 166.7,164.1,160.3,130.3,129.6,114.7,106.9$, 94.7, 55.7, 52.0, 27.4, 24.9; FTIR (thin film) 3102, 2998, 2944, 2842, 2360, 1726, 1637 , 1595, 1578, 1498, 1461, 1441, 1391, 1377, 1319, 1299, 1263, 1204, 1146, 1135, 1089, $1017,903 \mathrm{~cm}^{-1}$. Exact mass calculated for $\mathrm{C}_{15} \mathrm{H}_{18} \mathrm{O}_{6} \mathrm{~S}+\mathrm{Na}^{+}$(ES) 349.0700, found 349.0708 . 

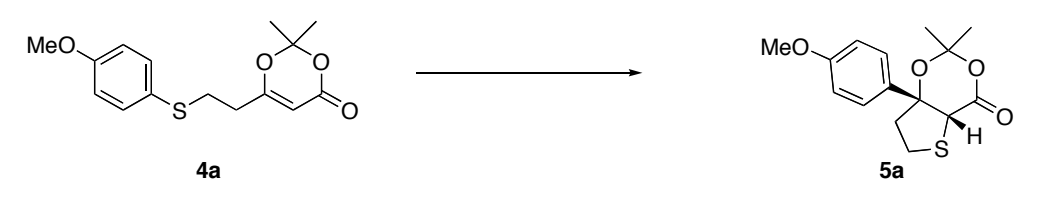

Thiolane photoadduct 5a. In a oven-dried $250 \mathrm{~mL}$ photoreactor with a Pyrex ${ }^{\mathrm{TM}}$ insert was added 6-[2-(4-Methoxy-phenylsulfanyl)-ethyl]-2,2-dimethyl-[1,3]dioxen-4-one 4a (200 $\mathrm{mg}, 0.68 \mathrm{mmol}$ ) with a 9:1 mixture of pre-irradiated and distilled acetonitrile and freshly distilled acetone $(225.0 \mathrm{~mL} / 25.0 \mathrm{~mL}, 2.7 \mathrm{mM})$. The mixture was purged at $0{ }^{\circ} \mathrm{C}$ under argon for $1 \mathrm{~h}$. The reaction was irradiated for $2 \mathrm{~h}$ and concentrated. Purification by silica gel chromatography (15\% ether/hexane) provided thiolane photoadduct $\mathbf{5 a}$ (105 $\mathrm{mg}, 53 \%)$ as a white solid, mp $108-111^{\circ} \mathrm{C}$ : ${ }^{1} \mathrm{H}$ NMR $(500 \mathrm{MHz}) \delta 7.42(\mathrm{~d}, J=9.0 \mathrm{~Hz}$, $2 \mathrm{H}), 6.89(\mathrm{~d}, J=8.8 \mathrm{~Hz}, 2 \mathrm{H}), 4.45(\mathrm{~s}, 1 \mathrm{H}), 3.80(\mathrm{~s}, 3 \mathrm{H}), 3.02(\mathrm{ddd}, J=10.3,6.3,6.3 \mathrm{~Hz}$, 1H), 2.68 (ddd, $J=10.3,7.1,7.1 \mathrm{~Hz}, 1 \mathrm{H}), 2.56$ (ddd, $J=12.9,7.2,6.0 \mathrm{~Hz}, 1 \mathrm{H}), 2.18$ (ddd, $J=12.6,6.2,6.2 \mathrm{~Hz}, 1 \mathrm{H}), 1.63(\mathrm{~s}, 3 \mathrm{H}), 1.10(\mathrm{~s}, 3 \mathrm{H}) ;{ }^{13} \mathrm{C} \mathrm{NMR}(125 \mathrm{MHz}) \delta 167.7$, 159.6, 134.0, 126.5, 114.1, 107.1, 87.0, 55.2, 48.0, 47.8, 29.8, 28.1, 27.1; FTIR (thin film) 2997, 2938, 2837, 2360, 2340, 2049, 1736, 1610, 1582, 1513, 1461, 1441, 1386, $1299,1280,1256,1233,1183,1111,1033,990,833 \mathrm{~cm}^{-1}$. Exact mass calculated for $\mathrm{C}_{15} \mathrm{H}_{18} \mathrm{O}_{4} \mathrm{~S}+\mathrm{Na}^{+}$(ES) 317.0826, found 317.0833.
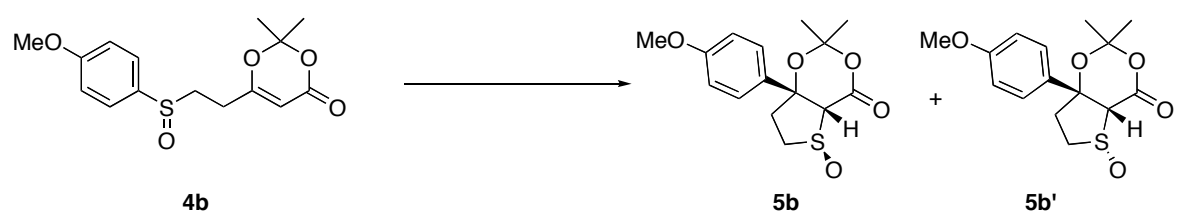

Thiolane 1-oxide 5b (Diast. 1) and Thiolane 1-oxide 5b' (Diast. 2). In a oven-dried 60 $\mathrm{mL}$ Pyrex $^{\mathrm{TM}}$ photoreactor was added 6-[2-(4-Methoxy-benzenesulfinyl)-ethyl]-2,2dimethyl-[1,3]dioxen-4-one $\mathbf{4 b}(41 \mathrm{mg}, 0.13 \mathrm{mmol})$ with a 9:1 mixture of pre-irradiated and distilled acetonitrile and freshly distilled acetone $(54.0 \mathrm{~mL} / 6.0 \mathrm{~mL}, 2.16 \mathrm{mM})$. The mixture was purged at $0{ }^{\circ} \mathrm{C}$ under argon for $1 \mathrm{~h}$. The reaction was irradiated for $15 \mathrm{~min}$ and concentrated. Purification by silica gel chromatography (33.3\% ethyl acetate/hexane to $66.7 \%$ ethyl acetate) provided two diastereomers, thiolane 1-oxide $\mathbf{5 b}$ diast. 1 (28 $\mathrm{mg}$, $54 \%)$ as a foam: ${ }^{1} \mathrm{H}$ NMR $(500 \mathrm{MHz}) \delta 7.39(\mathrm{~d}, J=9.0 \mathrm{~Hz}, 2 \mathrm{H}), 6.91(\mathrm{~d}, J=8.8 \mathrm{~Hz}$, $2 \mathrm{H}), 4.42(\mathrm{~s}, 1 \mathrm{H}), 3.81(\mathrm{~s}, 3 \mathrm{H}), 3.19(\mathrm{ddd}, J=13.2,11.8,6.3 \mathrm{~Hz}, 1 \mathrm{H}), 3.00(\mathrm{~m}, 1 \mathrm{H}), 2.90$ (ddd, $J=14.1,11.8,5.6 \mathrm{~Hz}, 1 \mathrm{H}), 2.84(\mathrm{ddd}, J=14.1,6.2,3.1 \mathrm{~Hz}, 1 \mathrm{H}), 1.60$ (s, 3H), 1.11 (s, 3H); ${ }^{13} \mathrm{C}$ NMR (125 MHz) $\delta 162.6,159.7,134.1,126.2,114.2,106.8,85.9,74.0,55.3$, 51.1, 45.5, 29.6, 27.9; FTIR (thin film) 2990, 2940, 2837, 2358, 1731, 1610, 1514, 1462, $1414,1379,1288,1255,1216,1184,1111,1041,969,867,833,809,732 \mathrm{~cm}^{-1}$. Exact mass calculated for $\mathrm{C}_{15} \mathrm{H}_{18} \mathrm{O}_{5} \mathrm{~S}+\mathrm{Na}^{+}$(ES) 333.0775, found 333.0764.

Thiolane 1-oxide 5b' diast. 2 (24 mg, 44\%) as a foam: ${ }^{1} \mathrm{H}$ NMR $(500 \mathrm{MHz}) \delta 7.29(\mathrm{~d}, J$ $=8.8 \mathrm{~Hz}, 2 \mathrm{H}), 6.90(\mathrm{~d}, J=8.8 \mathrm{~Hz}, 2 \mathrm{H}), 4.48(\mathrm{~s}, 1 \mathrm{H}), 3.81(\mathrm{~s}, 3 \mathrm{H}), 3.73(\mathrm{ddd}, J=12.6$, 6.9, $2.7 \mathrm{~Hz}, 1 \mathrm{H}), 3.25(\mathrm{ddd}, J=12.4,12.4,7.0 \mathrm{~Hz}, 1 \mathrm{H}), 3.08(\mathrm{ddd}, J=14.4,6.9,2.7 \mathrm{~Hz}$, $1 \mathrm{H}), 2.04(\mathrm{ddd}, J=14.4,12.1,6.9 \mathrm{~Hz}, 1 \mathrm{H}), 1.66(\mathrm{~s}, 3 \mathrm{H}), 0.97(\mathrm{~s}, 3 \mathrm{H}) ;{ }^{13} \mathrm{C} \mathrm{NMR}(125$ MHz) $\delta 160.3,160.2$, 132.1, 127.1, 114.5, 108.8, 84.7, 65.0, 55.4, 53.7, 42.7, 30.4, 28.1; FTIR (thin film) 2982, 2938, 2841, 1727, 1610, 1514, 1462, 1386, 1303, 1259, 1185, 
1124, 1092, 1047, 977, 834, 816, $728 \mathrm{~cm}^{-1}$. Exact mass calculated for $\mathrm{C}_{15} \mathrm{H}_{18} \mathrm{O}_{5} \mathrm{~S}+\mathrm{Na}^{+}$ (ES) 333.0775 , found 333.0758 .
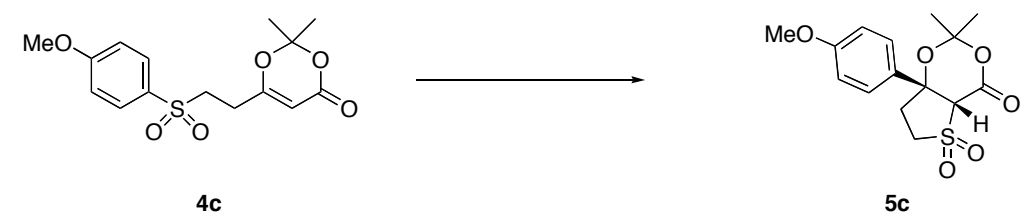

Thiolane 1,1-dioxide 5c. In a oven-dried $60 \mathrm{~mL} \mathrm{Pyrex}^{\mathrm{TM}}$ photoreactor was added 6-[2(4-Methoxy-benzenesulfonyl)-ethyl]-2,2-dimethyl-[1,3]dioxen-4-one 4c (45 mg, 0.14 mmol) with a 9:1 mixture of pre-irradiated and distilled acetonitrile and freshly distilled acetone $(54.0 \mathrm{~mL} / 6.0 \mathrm{~mL}, 2.30 \mathrm{mM})$. The mixture was purged at $0{ }^{\circ} \mathrm{C}$ under argon for 1 $\mathrm{h}$. The reaction was irradiated for $15 \mathrm{~min}$ and concentrated. Purification by silica gel chromatography (25\% ethyl acetate/hexane) provided thiolane 1,1-dioxide 5c $(10 \mathrm{mg}$, $22 \%)$ as a foam: ${ }^{1} \mathrm{H}$ NMR $(500 \mathrm{MHz}) \delta 7.27(\mathrm{~d}, J=9.0 \mathrm{~Hz}, 2 \mathrm{H}), 6.93(\mathrm{~d}, J=8.9 \mathrm{~Hz}$, $2 \mathrm{H}), 4.64(\mathrm{~s}, 1 \mathrm{H}), 3.64(\mathrm{~s}, 3 \mathrm{H}), 3.63(\mathrm{ddd}, J=12.6,12.6,7.1 \mathrm{~Hz}, 1 \mathrm{H}), 3.45$ (ddd, $J=$ $12.3,7.1,0.8 \mathrm{~Hz}, 1 \mathrm{H}$ ), 2.91 (ddd, $J=14.3,7.1,1.0 \mathrm{~Hz}, 1 \mathrm{H}$ ), 2.25 (ddd, $J=13.3,13.3,7.5$ $\mathrm{Hz}, 1 \mathrm{H}), 1.64$ (s, 3H), 0.94 (s, 3H); ${ }^{13} \mathrm{C}$ NMR $(125 \mathrm{MHz}) \delta 160.6,158.4,130.8,126.7$, 114.7, 108.8, 82.0, 66.3, 55.4, 51.8, 37.1, 29.9, 27.8; FTIR (thin film) 2938, 2849, 1735, $1611,1516,1463,1388,1333,1319,1259,1180,1154,1121,1088,976,834,732 \mathrm{~cm}^{-1}$. Exact mass calculated for $\mathrm{C}_{15} \mathrm{H}_{18} \mathrm{O}_{6} \mathrm{~S}+\mathrm{Na}^{+}$(ES) 349.0700, found 349.0714.
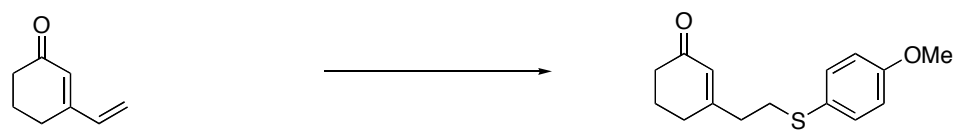

3-[2-(4-Methoxy-phenylsulfanyl)-ethyl]-cyclohex-2-enone. To a flask containing 3vinyl-cyclohex-2-enone $(200 \mathrm{mg}, 1.6 \mathrm{mmol})$ was added anhydrous benzene $(3.2 \mathrm{~mL}, 0.5$ M) and cooled to $0{ }^{\circ} \mathrm{C}$. 4-methoxybenzene thiol $(0.200 \mathrm{~mL}, 1.6 \mathrm{mmol})$ was next added followed by triethylamine $(0.070 \mathrm{~mL}, 0.3 \mathrm{mmol})$. The reaction was gradually warmed to $25{ }^{\circ} \mathrm{C}$ and stirred for $18 \mathrm{~h}$. The reaction was then quenched with $\mathrm{pH} 7$ buffer and extracted with ethyl acetate, dried, filtered, and concentrated. Purification by silica gel chromatography (25\% ethyl acetate/ hexanes) afforded 3-[2-(4-Methoxyphenylsulfanyl)-ethyl]-cyclohex-2-enone (312 mg, $74 \%)$ as an oil: ${ }^{1} \mathrm{H}$ NMR $(500 \mathrm{MHz})$ $\delta 7.34(\mathrm{~d}, J=7.9 \mathrm{~Hz}, 2 \mathrm{H}), 6.84(\mathrm{~d}, J=7.9 \mathrm{~Hz}, 2 \mathrm{H}), 5.85(\mathrm{~s}, 1 \mathrm{H}), 3.78$ (s, 3H), 2.94 (t, $J=$ $7.4 \mathrm{~Hz}, 2 \mathrm{H}), 2.45(\mathrm{t}, J=7.4 \mathrm{~Hz}, 2 \mathrm{H}), 2.33(\mathrm{t}, J=5.8 \mathrm{~Hz}, 2 \mathrm{H}), 2.24(\mathrm{t}, J=5.8 \mathrm{~Hz}, 2 \mathrm{H})$, 1.95 (dddd, $J=5.4,5.4,5.4,5.4 \mathrm{~Hz}, 2 \mathrm{H}) ;{ }^{13} \mathrm{C}$ NMR $(125 \mathrm{MHz}) \delta 199.5,163.5 .159 .2$, 133.8, 126.6, 125.4, 114.7, 55.3, 37.6, 37.2, 33.1, 29.4, 22.5; FTIR (thin film) 2939, $2834,1667,1625,1591,1493,1458,1284,1245,1178,1029,965,886,828 \mathrm{~cm}^{-1}$. Exact mass calculated for $\mathrm{C}_{15} \mathrm{H}_{18} \mathrm{O}_{2} \mathrm{~S}+\mathrm{Na}^{+}$(ES) 285.0926, found 285.0928. 


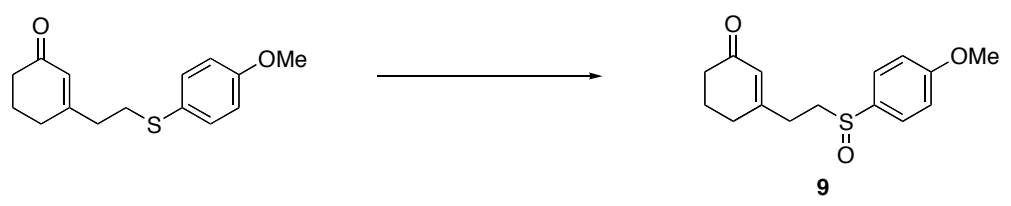

3-[2-(4-Methoxy-benzenesulfinyl)-ethyl]-cyclohex-2-enone 9. To a flask containing 3[2-(4-Methoxy-phenylsulfanyl)-ethyl]-cyclohex-2-enone (312 mg, $1.2 \mathrm{mmol})$ was added anhydrous methylene chloride $(60 \mathrm{~mL}, 0.02 \mathrm{M})$ and cooled to $-78{ }^{\circ} \mathrm{C}$. A solution of $\mathrm{m}$ CPBA (293 mg, $1.2 \mathrm{mmol}$ ) in anhydrous methylene chloride was added dropwise. The reaction was quenched in 10 min. with saturated $\mathrm{NaHCO}_{3}$ and extracted with methylene chloride $(3 x)$. The combined organic layers were washed with saturated $\mathrm{NaHCO}_{3}$ and dried, filtered, and concentrated. Purification by silica gel chromatography $(90 \%$ ethyl acetate/ heaxanes) afforded 3-[2-(4-Methoxy-benzenesulfinyl)-ethyl]-cyclohex-2-enone 9 (327 mg, 98\%) as an oil: ${ }^{1} \mathrm{H}$ NMR $(500 \mathrm{MHz}) \delta 7.50(\mathrm{~d}, J=8.9 \mathrm{~Hz}, 2 \mathrm{H}), 6.99(\mathrm{~d}, J=8.9$ $\mathrm{Hz}, 2 \mathrm{H}), 5.78(\mathrm{~s}, 1 \mathrm{H}), 3.81(\mathrm{~s}, 3 \mathrm{H}), 2.92(\mathrm{ddd}, J=13.1,10.3,5.8 \mathrm{~Hz}, 1 \mathrm{H}), 2.83$ (ddd, $J=$ $13.1,10.2,5.4 \mathrm{~Hz}, 1 \mathrm{H}), 2.59$ (ddd, $J=15.7,10.0,5.7 \mathrm{~Hz}, 1 \mathrm{H}), 2.40(\mathrm{~m}, 1 \mathrm{H}), 2.29$ (t, $J=$ $6.1 \mathrm{~Hz}, 2 \mathrm{H}$ ), 2.23 (ddd, $J=5.0,5.0,5.0 \mathrm{~Hz}, 2 \mathrm{H}), 1.92$ (dddd, $J=6.0,6.0,6.0,6.0 \mathrm{~Hz}$, $2 \mathrm{H}) ;{ }^{13} \mathrm{C}$ NMR $(125 \mathrm{MHz}) \delta 199.0,162.1,162.0,133.7,126.3,125.8,114.8,55.3,53.4$, 37.0, 29.6, 29.4, 22.4; FTIR (thin film) 3472, 2944, 1666, 1593, 1496, 1459, 1426, 1303, $1253,1186,1088,1031,833 \mathrm{~cm}^{-1}$. Exact mass calculated for $\mathrm{C}_{15} \mathrm{H}_{18} \mathrm{O}_{3} \mathrm{~S}+\mathrm{Na}^{+}$(ES) 301.0877, found 301.0885 .
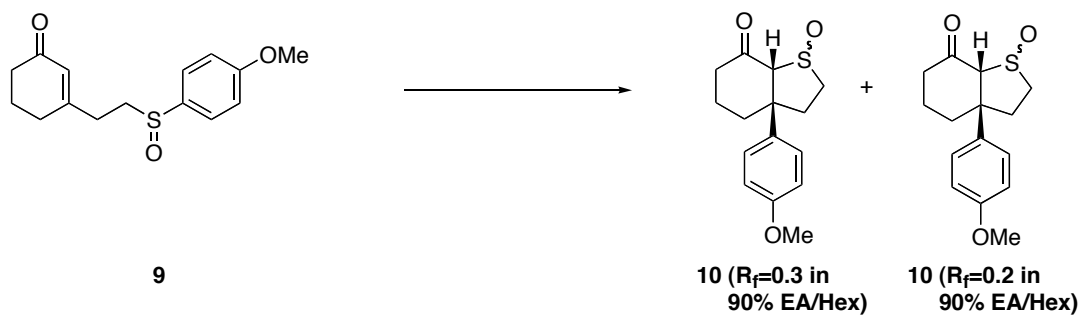

Thiolane 1-oxide photoadduct $10\left(R_{\mathrm{f}}=0.3\right.$ in $90 \%$ EA/Hex $)$ and thiolane 1-oxide photoadduct $10\left(\mathbf{R}_{\mathrm{f}}=\mathbf{0 . 2}\right.$ in $\left.\mathbf{9 0 \%} \mathbf{E A} / \mathbf{H e x}\right)$. In a oven-dried $60 \mathrm{~mL} \mathrm{Pyrex}^{\mathrm{TM}}$ photoreactor was added 3-[2-(4-Methoxy-benzenesulfinyl)-ethyl]-cyclohex-2-enone 9 (96 mg, 0.36 $\mathrm{mmol})$ with pre-irradiated and distilled acetonitrile $(60.0 \mathrm{~mL})$. The mixture was purged at $0{ }^{\circ} \mathrm{C}$ under argon for $1 \mathrm{~h}$. The reaction was irradiated for $20 \mathrm{~min}$ and concentrated. Purification by silica gel chromatography (66.6\% ethyl acetate/hexane to $90 \%$ ethyl acetate) provided two diastereomers, thiolane 1-oxide photoadduct $\mathbf{1 0}\left(\mathbf{R}_{\mathrm{f}}=\mathbf{0 . 3}\right.$ in $\mathbf{9 0 \%}$ ethyl acetate/hexane) $(40 \mathrm{mg}, 41 \%)$ as a foam: ${ }^{1} \mathrm{H}$ NMR $(500 \mathrm{MHz}) \delta 7.11(\mathrm{~d}, J=8.9$ $\mathrm{Hz}, 2 \mathrm{H}), 6.88(\mathrm{~d}, J=8.9 \mathrm{~Hz}, 2 \mathrm{H}), 3.82(\mathrm{~s}, 3 \mathrm{H}), 3.79(\mathrm{~m}, 1 \mathrm{H}), 3.77(\mathrm{~s}, 1 \mathrm{H}), 3.18$ (ddd, $J=$ 13.9, 10.9, 8.0 Hz, 1H), 2.96 (ddd, $J=13.8,8.0,3.0 \mathrm{~Hz}, 1 \mathrm{H}), 2.61$ (m, 2H), 2.44 (ddd, $J$ $=16.0,13.3,6.6 \mathrm{~Hz}, 1 \mathrm{H}), 2.12(\mathrm{~m}, 1 \mathrm{H}), 1.99(\mathrm{~m}, 1 \mathrm{H}), 1.87$ (ddddd, $J=13.3,6.2,3.3,3.3$, $3.3 \mathrm{~Hz}, 1 \mathrm{H}), 1.47$ (ddddd, $J=13.4,13.4,13.4,3.7,3.7 \mathrm{~Hz}, 1 \mathrm{H}) ;{ }^{13} \mathrm{C}$ NMR $(125 \mathrm{MHz})$ $\delta 204.6,158.4,136.7,127.1,114.1,76.4,56.4,55.4,55.3,43.6,41.6,33.9,20.8$; FTIR (thin film) 3375, 2939, 2873, 1698, 1609, 1513, 1456, 1411, 1300, 1254, 1222, 1187 , $1122,1085,1030,830 \mathrm{~cm}^{-1}$. Exact mass calculated for $\mathrm{C}_{15} \mathrm{H}_{18} \mathrm{O}_{3} \mathrm{~S}^{+}$(CI) 278.0977, found 278.0967. 
Thiolane 1-oxide photoadduct $\mathbf{1 0}\left(\mathbf{R}_{\mathbf{f}}=\mathbf{0 . 2}\right.$ in $\mathbf{9 0 \%}$ ethyl acetate/hexane) (38 $\left.\mathrm{mg}, 40 \%\right)$ as a foam: ${ }^{1} \mathrm{H}$ NMR $(500 \mathrm{MHz}) \delta 7.12(\mathrm{~d}, J=8.8 \mathrm{~Hz}, 2 \mathrm{H}), 6.87(\mathrm{~d}, J=8.8 \mathrm{~Hz}, 2 \mathrm{H}), 4.05$ $(\mathrm{s}, 1 \mathrm{H}), 3.82(\mathrm{~s}, 3 \mathrm{H}), 3.69(\mathrm{ddd}, J=14.0,10.9,7.7 \mathrm{~Hz}, 1 \mathrm{H}), 3.00(\mathrm{ddd}, J=14.0,8.8,2.5$ $\mathrm{Hz}, 1 \mathrm{H}), 2.73$ (ddd, $J=13.5,10.9,8.9 \mathrm{~Hz}, 1 \mathrm{H}), 2.65$ (ddd, $J=13.4,7.6,2.5 \mathrm{~Hz}, 1 \mathrm{H})$, $2.54(\mathrm{~m}, 1 \mathrm{H}), 2.45$ (ddd, $J=12.9,9.4,6.2 \mathrm{~Hz}, 1 \mathrm{H}), 1.97(\mathrm{~m}, 1 \mathrm{H}), 1.84$ (ddddd, $J=12.9$, 6.2, 3.1, 3.1, $3.1 \mathrm{~Hz}, 1 \mathrm{H}$ ), 1.74 (ddd, $J=13.0,13.0,3.3 \mathrm{~Hz}, 1 \mathrm{H}$ ), 1.49 (ddddd, $J=12.7$,

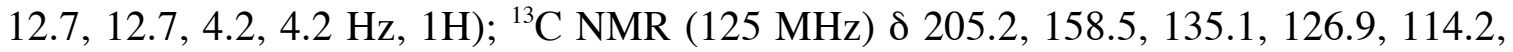
87.0, 55.9, 55.3, 52.4, 41.1, 39.5, 33.9, 21.2; FTIR (thin film) 3407, 2935, 2871, 1703, $1610,1514,1454,1417,1254,1188,1119,1031,947,831 \mathrm{~cm}^{-1}$. Exact mass calculated for $\mathrm{C}_{15} \mathrm{H}_{18} \mathrm{O}_{3} \mathrm{~S}^{+}(\mathrm{CI}) 278.0977$, found 278.0987.
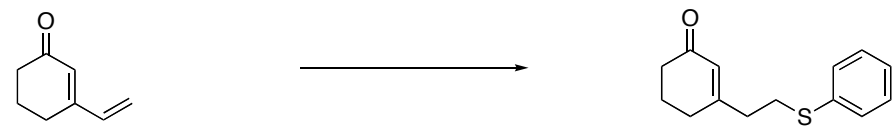

3-(2-Phenylsulfanyl-ethyl)-cyclohex-2-enone. To a flask containing 3-vinyl-cyclohex2-enone $(200 \mathrm{mg}, 1.6 \mathrm{mmol})$ was added anhydrous benzene $(3.2 \mathrm{~mL}, 0.5 \mathrm{M})$. The solution was cooled to $0{ }^{\circ} \mathrm{C}$ followed by the addition of benzenethiol $(0.168 \mathrm{~mL}, 1.6$ $\mathrm{mmol})$ and triethylamine $(0.067 \mathrm{~mL}, 0.48 \mathrm{mmol})$. The reaction was gradually warmed to $25{ }^{\circ} \mathrm{C}$ and quenched with $\mathrm{pH} 7$ buffer after $1.5 \mathrm{~h}$. The mixture was extracted with ethyl acetate $(3 \mathrm{x})$ and dried, filtered, and concentrated. Purification by silica gel chromatography (20\% ethyl acetate/ hexane) afforded 3-(2-Phenylsulfanyl-ethyl)cyclohex-2-enone $\left(315 \mathrm{mg}, 85 \%\right.$ ) as a white solid, 40-43 ${ }^{\circ} \mathrm{C}:{ }^{1} \mathrm{H}$ NMR (500 MHz) $\delta 7.34$ $(\mathrm{m}, 2 \mathrm{H}), 7.29(\mathrm{~m}, 2 \mathrm{H}), 7.21(\mathrm{~m}, 1 \mathrm{H}), 5.89(\mathrm{~s}, 1 \mathrm{H}), 3.07(\mathrm{t}, J=5.2 \mathrm{~Hz}, 2 \mathrm{H}), 2.52(\mathrm{t}, J=7.3$ $\mathrm{Hz}, 2 \mathrm{H}), 2.35(\mathrm{t}, J=5.3 \mathrm{~Hz}, 2 \mathrm{H}), 2.28(\mathrm{t}, J=5.5 \mathrm{~Hz}, 2 \mathrm{H}), 1.98$ (dddd, $J=4.0,4.0,4.0$, $4.0 \mathrm{~Hz}, 2 \mathrm{H}) ;{ }^{13} \mathrm{C}$ NMR $(125 \mathrm{MHz}) \delta 199.4,163.2,135.5,129.9,129.0,126.7,126.5,37.4$, 37.2, 31.2, 29.5, 22.5; FTIR (thin film) 3053, 2931, 2871, 1667, 1626, 1582, 1479, 1433, 1374, 1347, 1324, 1250, 1190, 1126, 1087, 1024, 965, 886, 741, $693 \mathrm{~cm}^{-1}$. Exact mass calculated for $\mathrm{C}_{14} \mathrm{H}_{16} \mathrm{OS}^{+}$(CI) 232.0922, found 232.0926.
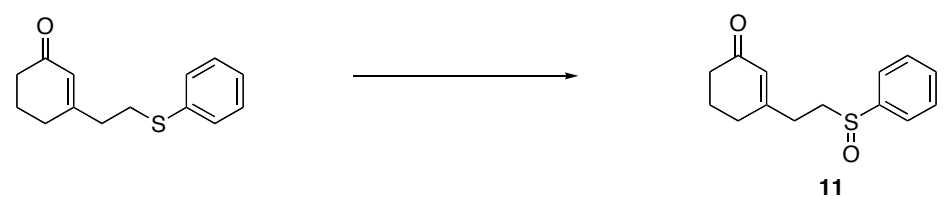

3-(2-Benzenesulfinyl-ethyl)-cyclohex-2-enone 11. To a flask containing enone 3-(2Phenylsulfanyl-ethyl)-cyclohex-2-enone $(280 \mathrm{mg}, 1.2 \mathrm{mmol})$ was added anhydrous methylene chloride $(60.0 \mathrm{~mL}, 0.02 \mathrm{M})$. The resulting solution was cooled to $-78{ }^{\circ} \mathrm{C}$. A solution of $m$-CPBA ( $297 \mathrm{mg}, 1.2 \mathrm{mmol}$ ) in methylene chloride was added to the cooled solution. The reaction was quenched in 10 min. with saturated $\mathrm{NaHCO}_{3}$ and extracted with methylene chloride $(3 \mathrm{x})$. The combined organic layers were washed with saturated $\mathrm{NaHCO}_{3}$ and dried, filtered, and concentrated. Purification by silica gel chromatography (90\% ethyl acetate/ heaxanes) afforded 3-(2-Benzenesulfinyl-ethyl)-cyclohex-2-enone 11 (266 mg, 89\%) as an oil: ${ }^{1} \mathrm{H}$ NMR $(500 \mathrm{MHz}) \delta 7.61(\mathrm{~m}, 2 \mathrm{H}), 7.52(\mathrm{~m}, 3 \mathrm{H}), 5.83(\mathrm{~s}, 1 \mathrm{H})$, 
3.02 (ddd, $J=13.1,9.5,5.7 \mathrm{~Hz}, 1 \mathrm{H}), 2.86$ (ddd, $J=13.1,10.3,5.2 \mathrm{~Hz}, 1 \mathrm{H}), 2.68$ (ddd, $J$ $=15.8,10.3,5.6 \mathrm{~Hz}, 1 \mathrm{H}), 2.44(\mathrm{ddd}, J=15.7,10.4,5.0 \mathrm{~Hz}, 1 \mathrm{H}), 2.33(\mathrm{t}, J=7.0 \mathrm{~Hz}, 2 \mathrm{H})$, 2.27 (ddd, $J=5.3,5.3,5.3 \mathrm{~Hz}, 2 \mathrm{H}$ ), 1.97 (dddd, $J=6.4,6.4,6.4,6.4 \mathrm{~Hz}, 2 \mathrm{H}$ ); ${ }^{13} \mathrm{C} \mathrm{NMR}$ (125 MHz) $\delta$ 199.0, 162.1, 143.0, 131.2, 129.4, 126.5, 123.9, 53.4, 37.1, 29.7, 29.3, 22.5; FTIR (thin film) 3457, 3056, 2941, 2876, 2360, 1665, 1441, 1377, 1319, 1250, 1187 , $1085,1041,750 \mathrm{~cm}^{-1}$. Exact mass calculated for $\mathrm{C}_{14} \mathrm{H}_{16} \mathrm{O}_{2} \mathrm{~S}+\mathrm{Na}^{+}$(ES) 271.0771, found 271.0759 .
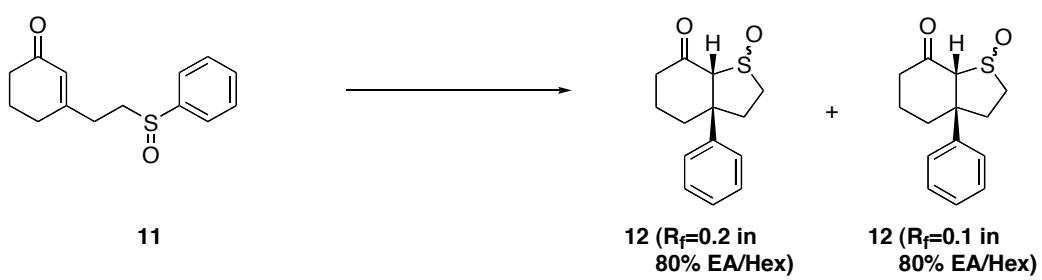

Thiolane 1-oxide photoadduct $12\left(R_{\mathrm{f}}=0.2\right.$ in $80 \%$ EA/Hex $)$ and thiolane 1-oxide photoadduct $12\left(\mathbf{R}_{\mathrm{f}}=\mathbf{0 . 1}\right.$ in $\left.\mathbf{8 0} \% \mathbf{E A} / \mathbf{H e x}\right)$. In a oven-dried $60 \mathrm{~mL} \mathrm{Pyrex}^{\mathrm{TM}}$ photoreactor was added 3-(2-Benzenesulfinyl-ethyl)-cyclohex-2-enone 11 (96 mg, $0.36 \mathrm{mmol})$ with pre-irradiated and distilled acetonitrile $(60.0 \mathrm{~mL})$. The mixture was purged at $0{ }^{\circ} \mathrm{C}$ under argon for $1 \mathrm{~h}$. The reaction was irradiated for $20 \mathrm{~min}$ and concentrated. Purification by silica gel chromatography ( $80 \%$ ethyl acetate/hexane to $100 \%$ ethyl acetate) provided two diastereomers thiolane 1 -oxide $12\left(\mathbf{R}_{\mathrm{f}}=\mathbf{0 . 2}\right.$ in $\mathbf{8 0 \%}$ ethyl acetate/hexane) (40 $\left.\mathrm{mg}, 41 \%\right)$ as a foam: ${ }^{1} \mathrm{H}$ NMR $(500 \mathrm{MHz}) \delta 7.35(\mathrm{~m}, 2 \mathrm{H}), 7.25(\mathrm{~m}, 1 \mathrm{H}), 7.17(\mathrm{~m}, 2 \mathrm{H}), 3.78(\mathrm{~s}, 1 \mathrm{H})$, $3.75(\mathrm{~m}, 1 \mathrm{H}), 3.16(\mathrm{ddd}, J=13.9,10.7,8.0 \mathrm{~Hz}, 1 \mathrm{H}), 2.98(\mathrm{ddd}, J=13.8,8.0,3.1 \mathrm{~Hz}$, $1 \mathrm{H}), 2.64(\mathrm{~m}, 2 \mathrm{H}), 2.45$ (ddd, $J=16.1,13.3,6.6 \mathrm{~Hz}, 1 \mathrm{H}), 2.16(\mathrm{~m}, 1 \mathrm{H}), 2.05(\mathrm{~m}, 1 \mathrm{H})$, 1.88 (ddddd, $J=13.4,6.2,3.4,3.4,3.4 \mathrm{~Hz}, 1 \mathrm{H}$ ), 1.46 (ddddd, $J=13.5,13.5,13.5,3.7$, $3.7 \mathrm{~Hz}, 1 \mathrm{H}) ;{ }^{13} \mathrm{C}$ NMR $(125 \mathrm{MHz}) \delta 204.5,144.7,128.9,127.1,126.0,76.2,57.0,55.2$, 43.6, 41.4, 34.0, 20.8; FTIR (thin film) 2942, 2866, 1698, 1596, 1491, 1445, 1408, 1320, $1272,1222,1123,1030 \mathrm{~cm}^{-1}$. Exact mass calculated for $\mathrm{C}_{14} \mathrm{H}_{16} \mathrm{O}_{2} \mathrm{~S}^{+}$(CI) 248.0871, found 248.0875 .

Thiolane 1-oxide $12\left(\mathbf{R}_{\mathbf{f}}=\mathbf{0 . 1}\right.$ in $\mathbf{8 0 \%}$ ethyl acetate/hexane) $(38 \mathrm{mg}, 40 \%)$ as a foam: ${ }^{1} \mathrm{H}$ NMR (500 MHz) $\delta 7.34(\mathrm{~m}, 2 \mathrm{H}), 7.27(\mathrm{~m}, 1 \mathrm{H}), 7.20(\mathrm{~m}, 2 \mathrm{H}), 4.05(\mathrm{~s}, 1 \mathrm{H}), 3.70$ (ddd, $J=$ 14.0, 10.8, 7.7 Hz, 1H), 3.01 (ddd, $J=14.0,8.6,2.7 \mathrm{~Hz}, 1 \mathrm{H}), 2.78$ (ddd, $J=13.5,10.8$, $8.7 \mathrm{~Hz}, 1 \mathrm{H}), 2.70(\mathrm{ddd}, J=13.5,7.6,2.6 \mathrm{~Hz}, 1 \mathrm{H}), 2.55(\mathrm{~m}, 1 \mathrm{H}), 2.46(\mathrm{ddd}, J=15.8$, 13.0, 6.1 Hz, 1H), 2.02 (m, 1H), 1.85 (ddddd, $J=13.0,6.3,3.3,3.3,3.3 \mathrm{~Hz}, 1 \mathrm{H}), 1.77$ (ddd, $J=12.9,12.9,3.3 \mathrm{~Hz}, 1 \mathrm{H}), 1.48$ (dddd, $J=13.3,13.3,13.3,4.3,4.3 \mathrm{~Hz}, 1 \mathrm{H}) ;{ }^{13} \mathrm{C}$ NMR $(125 \mathrm{MHz}) \delta 205.1,143.2,128.9,127.3,125.8,86.9,56.3,52.3,40.9,39.5,34.0$, 21.1; FTIR (thin film) 2939, 2872, 1702, 1597, 1495, 1446, 1417, 1315, 1270, 1221, $1160,1119,1044 \mathrm{~cm}^{-1}$. Exact mass calculated for $\mathrm{C}_{14} \mathrm{H}_{16} \mathrm{O}_{2} \mathrm{~S}-\mathrm{H}_{2} \mathrm{O}^{+}(\mathrm{CI}) 230.0747$, found 230.0758 . 

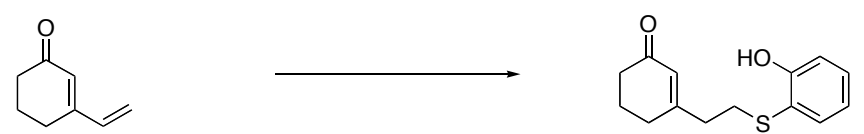

3-[2-(2-Hydroxy-phenylsulfanyl)-ethyl]-cyclohex-2-enone. To a flask containing 3vinyl-cyclohex-2-enone (100 mg, $0.82 \mathrm{mmol}$ ) was added anhydrous benzene (1.6 mL, 0.5 $\mathrm{M})$. The solution cooled to $0{ }^{\circ} \mathrm{C}$ followed by addition of 2-mercaptophenol $(0.082 \mathrm{~mL}$, $0.82 \mathrm{mmol})$ and triethylamine $(0.034 \mathrm{~mL}, 0.25 \mathrm{mmol})$. The reaction was quenched in 15 min. with $\mathrm{pH} 7$ buffer and extracted with ethyl acetate. The solution was dried and concentrated. Purification by silica gel chromatography (25\% hexane/ ethyl acetate) afforded 3-[2-(2-Hydroxy-phenylsulfanyl)-ethyl]-cyclohex-2-enone (180 $\mathrm{mg}, 88 \%)$ as a white solid, mp 120-121 ${ }^{\circ} \mathrm{C}$ : ${ }^{1} \mathrm{H}$ NMR $(500 \mathrm{MHz}) \delta 7.43(\mathrm{~d}, J=7.7 \mathrm{~Hz}, 1 \mathrm{H}), 7.26(\mathrm{t}, J$ $=8.3 \mathrm{~Hz}, 1 \mathrm{H}), 6.98(\mathrm{~d}, J=8.2 \mathrm{~Hz}, 1 \mathrm{H}), 6.87(\mathrm{t}, J=7.5 \mathrm{~Hz}, 1 \mathrm{H}), 6.71(\mathrm{~s}, 1 \mathrm{H}), 5.86(\mathrm{~s}$, $1 \mathrm{H}), 2.86(\mathrm{t}, J=7.3 \mathrm{~Hz}, 2 \mathrm{H}), 2.44(\mathrm{t}, J=7.5 \mathrm{~Hz}, 2 \mathrm{H}), 2.33(\mathrm{t}, J=6.2 \mathrm{~Hz}, 2 \mathrm{H}), 2.21(\mathrm{t}, J=$ $5.9 \mathrm{~Hz}, 2 \mathrm{H}), 1.95$ (dddd, $J=6.2,6.2,6.2,6.2 \mathrm{~Hz}, 2 \mathrm{H}) ;{ }^{13} \mathrm{C}$ NMR $(125 \mathrm{MHz}) \delta 199.4$, $162.8,157.0,135.8,131.3,126.7,120.9,118.1,115.0,37.6,37.2,33.2$, 29.4, 22.5; FTIR (thin film) 3135, 2945, 1632, 1576, 1448, 1382, 1260, 1224, 1126, 1070, 877, $748 \mathrm{~cm}^{-1}$. Exact mass calculated for $\mathrm{C}_{14} \mathrm{H}_{16} \mathrm{O}_{2} \mathrm{~S}+\mathrm{H}^{+}(\mathrm{CI}) 219.0888$, found 249.0940.
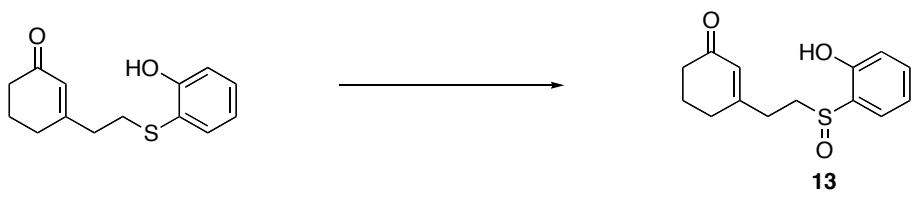

3-[2-(2-Hydroxy-benzenesulfinyl)-ethyl]-cyclohex-2-enone 13. To a flask containing 3-[2-(2-Hydroxy-phenylsulfanyl)-ethyl]-cyclohex-2-enone (133 mg, $0.54 \mathrm{mmol})$, was added anhydrous methylene chloride $(26.8 \mathrm{~mL}, 0.02 \mathrm{M})$ and the soluton was cooled to $-78{ }^{\circ} \mathrm{C}$. A solution of $m$-CPBA $(132 \mathrm{mg}, 1.0 \mathrm{mmol})$ in methylene chloride was added to the cooled solution. The reaction was quenched in $5 \mathrm{~min}$. with saturated $\mathrm{NaHCO}_{3}$ and extracted with methylene chloride $(3 \mathrm{x})$. The combined organic layers were washed with saturated $\mathrm{NaHCO}_{3}$ and dried, filtered, and concentrated. Purification by silica gel chromatography (10\% hexane/ethyl acetate) afforded 3-[2-(2-Hydroxy-benzenesulfinyl)ethyl]-cyclohex-2-enone 13 (103 mg, 72\%) as an oil: ${ }^{1} \mathrm{H}$ NMR (500 MHz) $\delta 10.03$ (s, $1 \mathrm{H}), 7.35(\mathrm{~m}, 1 \mathrm{H}), 7.25(\mathrm{dd}, J=7.7,1.5 \mathrm{~Hz}, 1 \mathrm{H}), 6.95(\mathrm{~m}, 2 \mathrm{H}), 5.88(\mathrm{~s}, 1 \mathrm{H}), 3.22(\mathrm{t}, J=$ $7.6 \mathrm{~Hz}, 2 \mathrm{H}), 2.73$ (ddd, $J=15.9,7.9,7.9 \mathrm{~Hz}, 1 \mathrm{H}), 2.58$ (ddd, $J=16.0,7.5,7.5 \mathrm{~Hz}, 1 \mathrm{H})$, $2.34(\mathrm{~m}, 2 \mathrm{H}), 2.29(\mathrm{~m}, 2 \mathrm{H}), 1.97$ (dddd, $J=6.1,6.1,6.1,6.1 \mathrm{~Hz}, 2 \mathrm{H}) ;{ }^{13} \mathrm{C}$ NMR $(125$ MHz) $\delta 199.5,162.3,157.8,133.0,126.4,125.3,122.6,120.1,118.4,50.9,37.1,29.7$, 29.7, 22.4; FTIR (thin film) 3063, 2935, 2698, 2572, 1668, 1586, 1451, 1373, 1295 , $1194,1127,1064,1002,885,832,757 \mathrm{~cm}^{-1}$. Exact mass calculated for $\mathrm{C}_{14} \mathrm{H}_{16} \mathrm{O}_{3} \mathrm{~S}+\mathrm{Na}^{+}$ (ES) 287.0720, found 287.0713. 


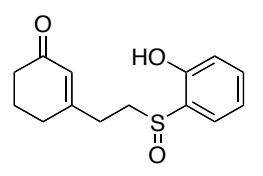

13

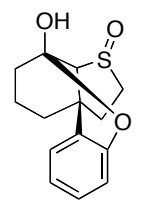

14

Hemiketal thiolane 1-oxide photoadduct 14. In an oven-dried $260 \mathrm{~mL}$ photoreactor with Pyrex ${ }^{\mathrm{TM}}$ insert was added 3-[2-(2-Hydroxy-benzenesulfinyl)-ethyl]-cyclohex-2enone $13(680 \mathrm{mg}, 2.57 \mathrm{mmol})$ with pre-irradiated and distilled acetonitrile $(260.0 \mathrm{~mL}$, $9.9 \mathrm{mM}$ ). The mixture was purged at $0{ }^{\circ} \mathrm{C}$ under argon for $1 \mathrm{~h}$. The reaction was irradiated for $85 \mathrm{~min}$ and concentrated to provide a clean crude mixture of hemiketal thiolane 1-oxide photoadducts $\mathbf{1 4}(665 \mathrm{mg}, 98 \%)$ as an oil: ${ }^{1} \mathrm{H} \mathrm{NMR}(500 \mathrm{MHz}) \delta 7.13$ (t, $J=7.7 \mathrm{~Hz}, 1 \mathrm{H}), 7.09(\mathrm{t}, J=7.6 \mathrm{~Hz}, 1 \mathrm{H}), 6.95(\mathrm{~d}, J=7.5 \mathrm{~Hz}, 1 \mathrm{H}), 6.92(\mathrm{~d}, J=7.5 \mathrm{~Hz}$, $1 \mathrm{H}), 6.85(\mathrm{~m}, 3 \mathrm{H}), 6.77(\mathrm{~d}, J=8.2 \mathrm{~Hz}, 1 \mathrm{H}), 6.36(\mathrm{~s}, 1 \mathrm{H}), 5.96(\mathrm{~s}, 1 \mathrm{H}), 3.87(\mathrm{~m}, 2 \mathrm{H}), 3.58$ (s, $1 \mathrm{H}), 3.14$ (ddd, $J=14.2,9.5,9.5 \mathrm{~Hz}, 1 \mathrm{H}), 2.99(\mathrm{t}, J=10.2 \mathrm{~Hz}, 1 \mathrm{H}), 2.80(\mathrm{dd}, J=$ 12.6, $8.9 \mathrm{~Hz}, 1 \mathrm{H}), 2.69(\mathrm{t}, J=10.4 \mathrm{~Hz}, 1 \mathrm{H}), 2.64(\mathrm{~s}, 1 \mathrm{H}), 2.51(\mathrm{~m}, 2 \mathrm{H}), 2.42$ (ddd, $J=$ 12.9, 12.9, $5.2 \mathrm{~Hz}, 1 \mathrm{H}), 2.16(\mathrm{~d}, J=13.2 \mathrm{~Hz}, 1 \mathrm{H}), 2.01(\mathrm{~m}, 3 \mathrm{H}), 1.63(\mathrm{~m}, 2 \mathrm{H}), 1.42(\mathrm{~m}$, $4 \mathrm{H}), 1.33(\mathrm{dd}, J=13.4,4.2 \mathrm{~Hz}, 1 \mathrm{H}) ;{ }^{13} \mathrm{C}$ NMR $(125 \mathrm{MHz}) \delta 155.1,155.0,128.7,128.5$, 128.4, 127.1, 125.0, 124.4, 120.6, 120.5, 115.3, 115.0, 100.2, 99.7, 77.0, 68.3, 56.9, 52.5, 51.1, 48.7, 36.4, 35.5, 34.8, 34.3, 33.3, 32.5, 19.5, 19.2; FTIR (thin film) 3209, 2941, 2874, 1578, 1483, 1451, 1352, 1296, 1233, 1178, 1162, 1103, 1034, 1015, 968, 912, 754, $732 \mathrm{~cm}^{-1}$. Exact mass calculated for $\mathrm{C}_{14} \mathrm{H}_{16} \mathrm{O}_{3} \mathrm{~S}^{+}$(CI) 264.0820, found 264.0818 .

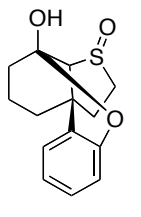

14

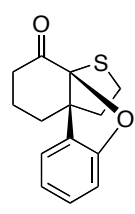

15

S, O ketal 15. The crude hemiketal thiolane 1-oxide photoadduct 14 (665 mg, $2.5 \mathrm{mmol})$ was then dissolved in anhydrous dichloromethane $(12.5 \mathrm{~mL}, 0.2 \mathrm{M})$ and cooled to $0{ }^{\circ} \mathrm{C}$. TFAA $(0.36 \mathrm{~mL}, 2.5 \mathrm{mmol})$ was added to the solution followed by TFA $(0.19 \mathrm{~mL}, 2.5$ $\mathrm{mmol})$. The reaction was stirred for $1 \mathrm{~h}$ and then concentrated. Purification by silica gel chromatography (50\% ethyl acetate/ hexanes) afforded S, O ketal $15(548 \mathrm{mg}, 89 \%)$ as an oil: ${ }^{1} \mathrm{H}$ NMR $(500 \mathrm{MHz}) \delta 7.20(\mathrm{dd}, J=7.2,7.2 \mathrm{~Hz}, 1 \mathrm{H}), 7.07(\mathrm{~d}, J=7.4 \mathrm{~Hz}, 1 \mathrm{H})$, $6.96(\mathrm{dd}, J=7.4,7.4 \mathrm{~Hz}, 1 \mathrm{H}), 6.92(\mathrm{~d}, J=7.9 \mathrm{~Hz}, 1 \mathrm{H}), 2.98(\mathrm{~m}, 2 \mathrm{H}), 2.72$ (ddd, $J=16.6$, 9.6, $6.3 \mathrm{~Hz}, 1 \mathrm{H}), 2.54(\mathrm{~m}, 2 \mathrm{H}), 2.42(\mathrm{ddd}, J=14.3,8.1,6.3 \mathrm{~Hz}, 1 \mathrm{H}), 2.10(\mathrm{~m}, 1 \mathrm{H}), 1.98$ (m, 2H), $1.70(\mathrm{~m}, 1 \mathrm{H}) ;{ }^{13} \mathrm{C}$ NMR $(125 \mathrm{MHz}) \delta 202.5,158.0,131.3,129.3,122.3,121.9$, 110.9, 109.2, 64.3, 45.1, 36.1, 32.4, 32.3, 19.6; FTIR (thin film) 2927, 1718, 1594, 1475, $1459,1223,986,912,856,752 \mathrm{~cm}^{-1}$. Exact mass calculated for $\mathrm{C}_{14} \mathrm{H}_{14} \mathrm{O}_{2} \mathrm{~S}^{+}(\mathrm{CI})$ 246.0715, found 246.0719. 


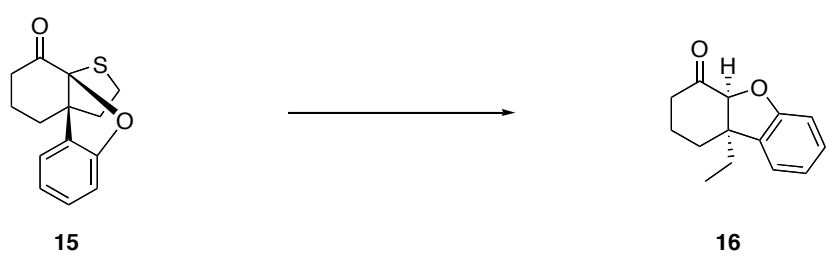

Dibenzofuran 16. A mixture of ethanol and methanol $(2 \mathrm{~mL}: 1 \mathrm{~mL}, 0.13 \mathrm{M})$ was added to $\mathrm{S}$, O ketal 15 (95 mg, $0.39 \mathrm{mmol})$. Raney nickel $(0.5 \mathrm{~mL}$, solution in water) was added to the solution and stirred for $24 \mathrm{~h}$. The mixture was put through celite and purified by silica gel chromatography ( $10 \%$ ether/ hexanes) to provide dibenzofuran 16 (49 $\mathrm{mg}$, $59 \%)$ as a solid, mp $70-72{ }^{\circ} \mathrm{C}:{ }^{1} \mathrm{H}$ NMR $\left(500 \mathrm{MHz}, \mathrm{CDCl}_{3}\right) \delta 7.17(\mathrm{~m}, 1 \mathrm{H}), 7.03(\mathrm{~m}, 1 \mathrm{H})$, $6.93(\mathrm{~m}, 2 \mathrm{H}), 4.54(\mathrm{~s}, 1 \mathrm{H}), 2.55$ (ddd, $J=15.1,4.8,4.8 \mathrm{~Hz}, 1 \mathrm{H}), 2.34(\mathrm{~m}, 1 \mathrm{H}), 2.02(\mathrm{~m}$, $1 \mathrm{H}), 1.93(\mathrm{~m}, 1 \mathrm{H}), 1.85(\mathrm{~m}, 1 \mathrm{H}), 1.76(\mathrm{~m}, 2 \mathrm{H}), 1.65(\mathrm{~m}, 1 \mathrm{H}), 0.85(\mathrm{t}, J=7.4 \mathrm{~Hz}, 3 \mathrm{H}) ;{ }^{13} \mathrm{C}$ NMR $\left(125 \mathrm{MHz}, \mathrm{CDCl}_{3}\right) \delta 208.7,159.5,131.4,128.7,122.8,121.3,110.2,89.9,53.9$, 38.3, 33.7, 32.0, 20.4, 8.4; FTIR (thin film) 2934, 1724, 1595, 1473, 1458, 1177, 997, $830,755 \mathrm{~cm}^{-1}$. Exact mass calculated for $\mathrm{C}_{14} \mathrm{H}_{16} \mathrm{O}_{2}{ }^{+}(\mathrm{CI}) 216.1150$, found 216.1140 .
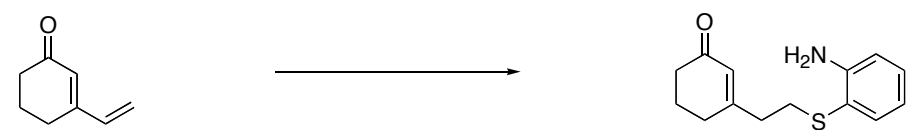

3-[2-(2-Amino-phenylsulfanyl)-ethyl]-cyclohex-2-enone. To a flask containing 3vinyl-cyclohex-2-enone $(417 \mathrm{mg}, 3.4 \mathrm{mmol})$ was added anhydrous benzene $(17 \mathrm{~mL}, 0.2$ $\mathrm{M})$ and the solution was cooled to $0{ }^{\circ} \mathrm{C}$. 2 -aminothiophenol $(0.37 \mathrm{~mL}, 3.4 \mathrm{mmol})$ was added followed by triethylamine $(0.142 \mathrm{~mL}, 1.0 \mathrm{mmol})$. The reaction was gradually warmed to $25^{\circ} \mathrm{C}$ and quenched with ph 7 buffer after $7 \mathrm{~h}$. The mixture was extracted with ethyl acetate (3x), dried, filtered, and concentrated to afford 3-[2-(2-Aminophenylsulfanyl)-ethyl]-cyclohex-2-enone ( $894 \mathrm{mg}$, quantitative) as an oil: ${ }^{1} \mathrm{H}$ NMR $\left(500 \mathrm{MHz}, \mathrm{CDCl}_{3}\right) \delta 7.34(\mathrm{dd}, J=7.6,1.1 \mathrm{~Hz}, 1 \mathrm{H}), 7.11$ (ddd, $\left.J=8.1,8.1,1.3 \mathrm{~Hz}, 1 \mathrm{H}\right)$, $6.71(\mathrm{~m}, 1 \mathrm{H}), 6.67(\mathrm{ddd}, J=7.6,7.6,1.1 \mathrm{~Hz}, 1 \mathrm{H}), 5.87(\mathrm{~s}, 1 \mathrm{H}), 4.34(\mathrm{~s}, 2 \mathrm{H}), 2.89(\mathrm{t}, J=$ $7.3 \mathrm{~Hz}, 2 \mathrm{H}), 2.44(\mathrm{t}, J=7.2 \mathrm{~Hz}, 2 \mathrm{H}), 2.31(\mathrm{t}, J=5.5 \mathrm{~Hz}, 2 \mathrm{H}), 1.93$ (dddd, $J=6.2,6.2$, $6.2,6.2 \mathrm{~Hz}, 2 \mathrm{H}) ;{ }^{13} \mathrm{C}$ NMR $\left(125 \mathrm{MHz}, \mathrm{CDCl}_{3}\right) \delta$ 199.5, 163.5, 148.4, 136.0, 130.0, 126.6, 118.4, 116.7, 114.9, 37.9, 37.2, 31.4, 29.3, 22.5; FTIR (thin film) 3442, 3343, 2924, $2882,1665,1609,1479,1447,1308,1254,1192,750 \mathrm{~cm}^{-1}$. Exact mass calculated for $\mathrm{C}_{14} \mathrm{H}_{17} \mathrm{ONS}^{+}(\mathrm{CI})$ 247.1031, found 247.1021.
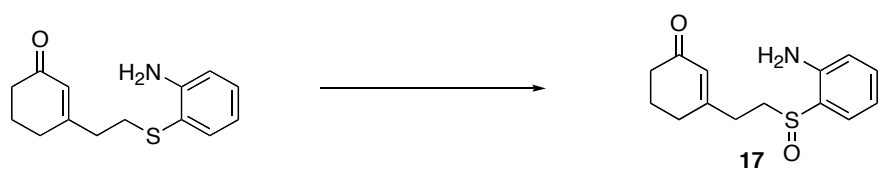

3-[2-(2-Amino-benzenesulfinyl)-ethyl]-cyclohex-2-enone 17. To a flask containing 3[2-(2-Amino-phenylsulfanyl)-ethyl]-cyclohex-2-enone $(99 \mathrm{mg}, 0.4 \mathrm{mmol})$ was added anhydrous methylene chloride $(2.0 \mathrm{~mL}, 0.2 \mathrm{M})$. The resulting solution was cooled to -78 ${ }^{\circ} \mathrm{C}$. A solution of $m$-CPBA ( $99 \mathrm{mg}, 0.4 \mathrm{mmol}$ ) in methylene chloride was added to the 
cooled solution. The reaction was quenched in 35 min. with saturated $\mathrm{NaHCO}_{3}$ and extracted with methylene chloride $(3 \mathrm{x})$. The combined organic layers were washed with saturated $\mathrm{NaHCO}_{3}$ and dried, filtered, and concentrated. Purification by silica gel chromatography (90\% ethyl acetate/ heaxanes) afforded 3-[2-(2-Amino-benzenesulfinyl)ethyl]-cyclohex-2-enone 17 (73 mg, 69\%) as a foam: ${ }^{1} \mathrm{H} \mathrm{NMR}\left(500 \mathrm{MHz}, \mathrm{CDCl}_{3}\right) \delta 7.23$ $(\mathrm{m}, 1 \mathrm{H}), 7.18(\mathrm{dd}, J=7.7,1.4 \mathrm{~Hz}, 1 \mathrm{H}), 6.73(\mathrm{ddd}, J=7.7,7.7,1.0 \mathrm{~Hz}, 1 \mathrm{H}), 6.67$ (dd, $J=$ $8.1,0.8 \mathrm{~Hz}, 1 \mathrm{H}), 5.86(\mathrm{~s}, 1 \mathrm{H}), 5.02(\mathrm{~s}, 2 \mathrm{H}), 3.42$ (ddd, $J=12.9,9.4,5.9 \mathrm{~Hz}, 1 \mathrm{H}), 3.17$ $(\mathrm{ddd}, J=12.8,9.4,6.6 \mathrm{~Hz}, 1 \mathrm{H}), 2.55(\mathrm{~m}, 2 \mathrm{H}), 2.32(\mathrm{dd}, J=7.6,6.0 \mathrm{~Hz}, 2 \mathrm{H}), 2.26(\mathrm{t}, J=$ $5.8 \mathrm{~Hz}, 2 \mathrm{H}), 1.96(\mathrm{~m}, 2 \mathrm{H}) ;{ }^{13} \mathrm{C} \mathrm{NMR}\left(125 \mathrm{MHz}, \mathrm{CDCl}_{3}\right) \delta$ 199.1, 161.9, 147.7, 132.7, 127.0, 126.4, 121.4, 117.6, 117.5, 47.9, 37.1, 30.9, 29.6, 22.4; FTIR (thin film) 3410, 3336, 3231, 2949, 2869, 1666, 1626, 1595, 1482, 1452, 1323, 1256, 1030, 1016, $755 \mathrm{~cm}^{-}$

${ }^{1}$. Exact mass calculated for $\mathrm{C}_{14} \mathrm{H}_{17} \mathrm{O}_{2} \mathrm{NS}^{+}(\mathrm{CI})$ 263.0980, found 263.0973.
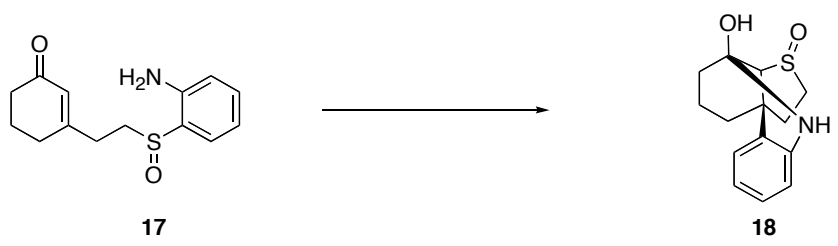

Hemi-aminal Thiolane 1-oxide Photoadduct 18. In an oven-dried $140 \mathrm{~mL}$ photoreactor with Pyrex ${ }^{\mathrm{TM}}$ insert was added 3-[2-(2-Amino-benzenesulfinyl)-ethyl]-cyclohex-2-enone $17(347 \mathrm{mg}, 1.24 \mathrm{mmol})$ with pre-irradiated and distilled acetonitrile $(130.0 \mathrm{~mL}, 9.6$ $\mathrm{mM})$. The mixture was purged at $0{ }^{\circ} \mathrm{C}$ under argon for $1 \mathrm{~h}$. The reaction was irradiated for $20 \mathrm{~min}$ and concentrated to afford a crude mixture of hemi-aminal thiolane 1-oxide photoadducts $18(345 \mathrm{mg}, 100 \%)$ as an oil: ${ }^{1} \mathrm{H} \mathrm{NMR}\left(500 \mathrm{MHz}, \mathrm{CDCl}_{3}\right) \delta 7.00(\mathrm{~m}, 2 \mathrm{H})$, $6.87(\mathrm{dd}, J=7.6,1.0 \mathrm{~Hz}, 1 \mathrm{H}), 6.81(\mathrm{dd}, J=7.6,1.1 \mathrm{~Hz}, 1 \mathrm{H}), 6.74(\mathrm{t}, J=7.4 \mathrm{~Hz}, 1 \mathrm{H})$, $6.64(\mathrm{~m}, 2 \mathrm{H}), 6.55(\mathrm{~d}, J=8.0 \mathrm{~Hz}, 1 \mathrm{H}), 5.90(\mathrm{~s}, 4 \mathrm{H}), 3.81(\mathrm{~m}, 2 \mathrm{H}), 3.55(\mathrm{~s}, 1 \mathrm{H}), 3.04$ (ddd, $J=14.3,9.0,9.0 \mathrm{~Hz}, 1 \mathrm{H}), 2.90(\mathrm{dd}, J=13.0,9.8 \mathrm{~Hz}, 1 \mathrm{H}), 2.71(\mathrm{dd}, J=12.2,9.2$ $\mathrm{Hz}, 1 \mathrm{H}), 2.62(\mathrm{~s}, 1 \mathrm{H}), 2.57(\mathrm{~m}, 2 \mathrm{H}), 2.44(\mathrm{~m}, 2 \mathrm{H}), 2.16(\mathrm{ddd}, J=13.5,13.5,4.9 \mathrm{~Hz}, 1 \mathrm{H})$, $1.97(\mathrm{~m}, 1 \mathrm{H}), 1.90(\mathrm{~d}, J=12.2 \mathrm{~Hz}, 1 \mathrm{H}), 1.82(\mathrm{~m}, 1 \mathrm{H}), 1.63(\mathrm{~m}, 2 \mathrm{H}), 1.53(\mathrm{~m}, 2 \mathrm{H}), 1.39$ $(\mathrm{d}, J=11.6 \mathrm{~Hz}, 1 \mathrm{H}), 1.33(\mathrm{~d}, J=12.5 \mathrm{~Hz}, 1 \mathrm{H}), 1.27(\mathrm{ddd}, J=12.8,12.8,4.6 \mathrm{~Hz}, 1 \mathrm{H}) ;{ }^{13} \mathrm{C}$ NMR $\left(125 \mathrm{MHz}, \mathrm{CDCl}_{3}\right) \delta 143.8,142.3,128.8,128.0,127.8,126.1,125.4,124.7,120.0$, $118.1,114.9,113.4,84.4,82.4,79.0,69.2$, 56.7, 52.4, 52.1, 49.6, 36.5, 36.4, 36.4, 35.0, 34.3, 33.3, 19.8, 1.8; FTIR (thin film) 3295, 2939, 2871, 2241, 1661, 1604, 1484, 1418, $1341,1301,1182,1145,1117,1091,1034,910,734 \mathrm{~cm}^{-1}$. Exact mass calculated for $\mathrm{C}_{14} \mathrm{H}_{17} \mathrm{O}_{2} \mathrm{NS}^{+}$(CI) 263.0980, found 263.0969.
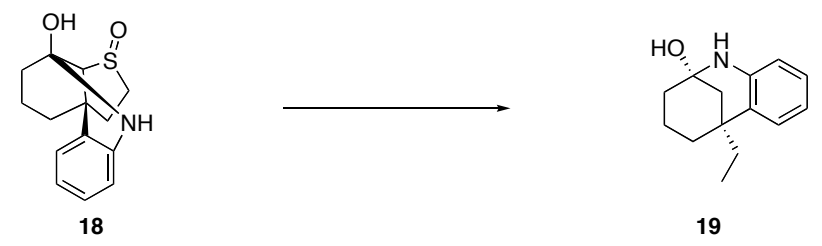

Hemiaminal Tricycle 19. To a flask containing hemi-aminal thiolane 1-oxide photoadduct 18 (126 mg, $0.45 \mathrm{mmol})$ was added a 3:1 mixture of 95\% methanol and 
anhydrous tetrahydrofuran $(11.3 \mathrm{~mL}, 3.75 \mathrm{~mL}, 0.03 \mathrm{M})$ at $0{ }^{\circ} \mathrm{C}$. Nickel chloride hexahydrate $(1.5 \mathrm{~g}, 6.3 \mathrm{mmol})$ was added as a solid. Sodium borohydride $(715 \mathrm{mg}, 18.9$ $\mathrm{mmol}$ ) was then added portionwise over $15 \mathrm{~min}$. The resulting black suspension was put through a plug of celite after an additional $15 \mathrm{~min}$. of stirring at $25{ }^{\circ} \mathrm{C}$. Purification by silica gel chromatography (5\% diethyl ether/ methylene chloride) afforded hemiaminal tricycle 19 (55 mg, 56\%) as a white solid, mp 85-90 ${ }^{\circ} \mathrm{C}:{ }^{1} \mathrm{H}$ NMR (500 $\mathrm{MHz}, \mathrm{CDCl}_{3}$ ) $\delta 6.99(\mathrm{~m}, 2 \mathrm{H}), 6.68(\mathrm{ddd}, J=7.5,7.5,1.1 \mathrm{~Hz}, 1 \mathrm{H}), 6.50(\mathrm{~m}, 1 \mathrm{H}), 2.01(\mathrm{~m}, 2 \mathrm{H}), 1.83(\mathrm{~m}$, $2 \mathrm{H}), 1.62(\mathrm{~m}, 3 \mathrm{H}), 1.45(\mathrm{~m}, 3 \mathrm{H}), 0.86(\mathrm{t}, J=7.4 \mathrm{~Hz}, 3 \mathrm{H}) ;{ }^{13} \mathrm{C}$ NMR $\left(125 \mathrm{MHz}, \mathrm{CDCl}_{3}\right)$ $\delta 145.5,126.9,126.6,125.3,117.9,113.5,80.8,42.5,41.0,40.9,40.4,31.5,20.4,7.9$; FTIR (thin film) 3357, 2934, 2865, 1606, 1478, 1451, 1298, 1139, 1085, 1058, 1044, 744 $\mathrm{cm}^{-1}$. Exact mass calculated for $\mathrm{C}_{14} \mathrm{H}_{19} \mathrm{ON}^{+}$(CI) 217.1467, found 217.1464.
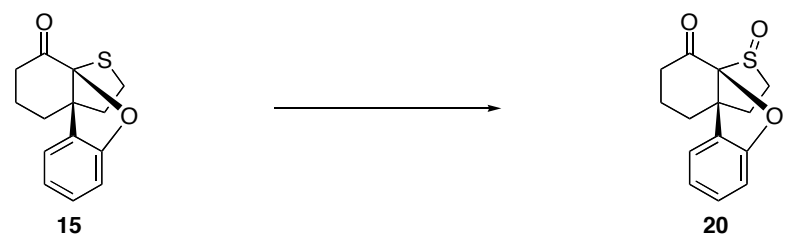

Thiolane 1-oxide $\mathbf{O}, \mathbf{S}$ Ketal 20. To a stirred solution of O, S ketal 15 (30 mg, 0.12 $\mathrm{mmol})$ in dichloromethane $(0.6 \mathrm{~mL}, 0.2 \mathrm{M}$ ) was added $m$-CPBA (30 $\mathrm{mg}$ of $70 \%, 0.12$ mmol) at $-78{ }^{\circ} \mathrm{C}$. The reaction was quenched after $10 \mathrm{~min}$. with saturated $\mathrm{NaHCO}_{3}$. The mixture was extracted $3 \mathrm{x}$ with dichloromethane. The combined organics were washed several times with saturated $\mathrm{NaHCO}_{3}$, dried, filtered, and concentrated. Purification by silica gel chromatography (33\% ethyl acetate/ hexanes) provided thiolane 1-oxide O, S ketal $20(26 \mathrm{mg}, 82 \%)$ as a foam: ${ }^{1} \mathrm{H}$ NMR (500 MHz) $\delta 7.19(\mathrm{~m}, 2 \mathrm{H}), 7.03$ (ddd, $J=$ $7.5,7.5,0.8 \mathrm{~Hz}, 1 \mathrm{H}), 6.88(\mathrm{~d}, J=8.1 \mathrm{~Hz}, 1 \mathrm{H}), 3.51$ (ddd, $J=13.8,13.8,4.9 \mathrm{~Hz}, 1 \mathrm{H})$, $3.07(\mathrm{dd}, J=13.3,4.2 \mathrm{~Hz}, 1 \mathrm{H}), 2.67(\mathrm{dd}, J=5.6,0.7 \mathrm{~Hz}, 1 \mathrm{H}), 2.56(\mathrm{~m}, 2 \mathrm{H}), 2.40$ (ddd, $J$ $=14.1,14.1,5.7 \mathrm{~Hz}, 1 \mathrm{H}), 2.32(\mathrm{ddd}, J=13.7,7.0,2.4 \mathrm{~Hz}, 1 \mathrm{H}), 2.23(\mathrm{~m}, 1 \mathrm{H}), 2.04$ (ddd, $J=13.7,13.7,2.8 \mathrm{~Hz}, 1 \mathrm{H}), 1.81(\mathrm{~m}, 1 \mathrm{H}) ;{ }^{13} \mathrm{C} \mathrm{NMR}(125 \mathrm{MHz}) \delta 203.5,156.1,131.6$, 129.6, 123.1, 122.8, 110.3, 109.5, 59.1, 49.1, 43.0, 40.1, 33.4, 18.6; FTIR (thin film) 2933, 1716, 1594, 1477, 1460, 1217, 1051, 1018, $754 \mathrm{~cm}^{-1}$. Exact mass calculated for $\mathrm{C}_{14} \mathrm{H}_{14} \mathrm{O}_{3} \mathrm{~S}^{+}(\mathrm{CI})$ 262.0664, found 262.0661.

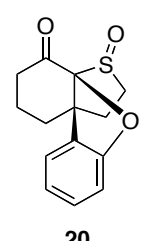

20

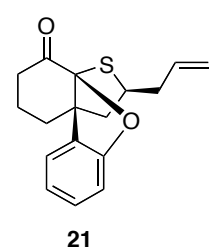

21

Allylated Thiolane 21. Anhydrous dichloromethane $(1.0 \mathrm{~mL}, 0.2 \mathrm{M})$ was added to a flask containing thiolane 1-oxide $\mathrm{O}, \mathrm{S}$ ketal $20(50 \mathrm{mg}, 0.19 \mathrm{mmol})$ and the resulting solution was cooled to $0{ }^{\circ} \mathrm{C}$. TFAA $(0.067 \mathrm{~mL}, 0.48 \mathrm{mmol})$ was added to the solution. The reaction was stirred for $10 \mathrm{~h}$ and then concentrated. Anhydrous dichloromethane $(1.0 \mathrm{~mL}, 0.2 \mathrm{M})$ was added to the crude material and the resulting solution was cooled to 
$-78{ }^{\circ} \mathrm{C}$. Allyltrimethylsilane $(0.067 \mathrm{~mL}, 0.42 \mathrm{mmol})$ was then added followed by $\mathrm{BF}_{3} \mathrm{OEt}_{2}(0.027 \mathrm{~mL}, 0.21 \mathrm{mmol})$. The reaction was warmed to $0{ }^{\circ} \mathrm{C}$ and then quenched after 30 min. with saturated $\mathrm{NaHPO}_{4}$. The mixture was extracted with dichloromethane, dried, filtered, and concentrated. Purification by silica gel chromatography (50\% dichloromethane/ pentanes) provided allylated thiolane $21(28 \mathrm{mg}, 51 \%)$ as an oil: ${ }^{1} \mathrm{H}$ NMR (500 MHz, CDCl $) \delta 7.19$ (ddd, $J=7.8,7.8,1.4 \mathrm{~Hz}, 1 \mathrm{H}), 7.03$ (dd, $J=7.7,1.4 \mathrm{~Hz}$, $1 \mathrm{H}), 6.93(\mathrm{~m}, 2 \mathrm{H}), 5.72$ (dddd, $J=17.1,10.3,6.9,6.9 \mathrm{~Hz}, 1 \mathrm{H}), 5.02(\mathrm{~m}, 2 \mathrm{H}), 3.87(\mathrm{~m}$, $1 \mathrm{H}), 2.83(\mathrm{ddd}, J=18.4,12.8,5.7 \mathrm{~Hz}, 1 \mathrm{H}), 2.42(\mathrm{~m}, 2 \mathrm{H}), 2.35(\mathrm{~m}, 2 \mathrm{H}), 2.23(\mathrm{~m}, 1 \mathrm{H})$, $2.08(\mathrm{~m}, 2 \mathrm{H}), 1.99(\mathrm{~m}, 1 \mathrm{H}), 1.62(\mathrm{~m}, 1 \mathrm{H}) ;{ }^{13} \mathrm{C} \mathrm{NMR}\left(125 \mathrm{MHz}, \mathrm{CDCl}_{3}\right) \delta 203.0,157.5$, 135.4, 131.5, 129.4, 121.6, 121.5, 117.4, 111.0, 108.2, 65.2, 50.9, 50.7, 40.1, 36.4, 30.0, 21.0; FTIR (thin film) 2923, 1718, 1594, 1474, 1459, 1222, 992, $917 \mathrm{~cm}^{-1}$. Exact mass calculated for $\mathrm{C}_{17} \mathrm{H}_{18} \mathrm{O}_{2} \mathrm{~S}+\mathrm{H}^{+}(\mathrm{CI})$ 287.1106, found 287.1099.
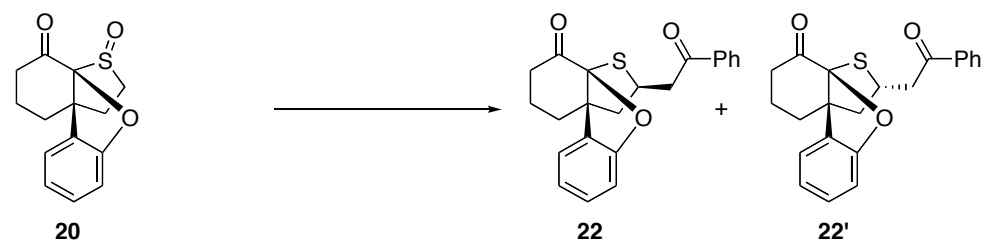

Diketone 22 (Diast. 1) and Diketone 22' (Diast. 2). To a flame-dried flask, was added thiolane 1-oxide $\mathrm{O}, \mathrm{S}$ ketal 20 (30 mg, $0.11 \mathrm{mmol})$. Anhydrous dichloromethane (1.1 $\mathrm{mL}, 0.2 \mathrm{M}$ ) was added to the solution and was cooled to $0{ }^{\circ} \mathrm{C}$. The silyl enol ether of acetophenone $(110 \mathrm{mg}, 0.57 \mathrm{mmol})$ was added followed by 2,6-lutidine $(0.066 \mathrm{~mL}, 0.57$ mmol). A slow addition of TMSOTf $(0.112 \mathrm{~mL}, 0.57 \mathrm{mmol})$ was next added. The reaction was stirred for $6 \mathrm{~h}$ and quenched saturated $\mathrm{NaHCO}_{3}$ and extracted with dichloromethane $(3 \mathrm{x})$, dried, filtered, and concentrated. Purification by silica gel chromatography (20\% ether/ hexanes) to afford a mixture of diastereomers (26 mg, 65\%) which was furthered choratographed (100\% dichloromethane) to provide Diketone 22, Dias. $1(20 \mathrm{mg}, 50 \%)$ as an oil: ${ }^{1} \mathrm{H}$ NMR $\left(500 \mathrm{MHz}, \mathrm{CDCl}_{3}\right) \delta 7.81(\mathrm{~d}, J=7.6 \mathrm{~Hz}, 2 \mathrm{H})$, $7.55(\mathrm{t}, J=7.2 \mathrm{~Hz}, 1 \mathrm{H}), 7.42(\mathrm{t}, J=7.6 \mathrm{~Hz}, 2 \mathrm{H}), 7.21(\mathrm{t}, J=7.9 \mathrm{~Hz}, 1 \mathrm{H}), 7.04(\mathrm{~d}, J=7.3$ $\mathrm{Hz}, 1 \mathrm{H}), 6.93(\mathrm{~m}, 2 \mathrm{H}), 4.35$ (dddd, $J=6.9,6.9,6.9,6.9 \mathrm{~Hz}, 1 \mathrm{H}), 3.31(\mathrm{~d}, J=6.8 \mathrm{~Hz}$, $2 \mathrm{H}), 2.90(\mathrm{~m}, 1 \mathrm{H}), 2.63(\mathrm{dd}, J=13.2,5.9 \mathrm{~Hz}, 1 \mathrm{H}), 2.42(\mathrm{~d}, J=15.7 \mathrm{~Hz}, 1 \mathrm{H}), 2.25(\mathrm{~d}, J=$ $14.3 \mathrm{~Hz}, 1 \mathrm{H}), 2.14(\mathrm{~m}, 2 \mathrm{H}), 2.02(\mathrm{dd}, J=8.8,4.5 \mathrm{~Hz}, 1 \mathrm{H}), 1.68(\mathrm{~m}, 1 \mathrm{H}) ;{ }^{13} \mathrm{C}$ NMR $(125$ $\mathrm{MHz}, \mathrm{CDCl}_{3}$ ) $\delta$ 202.9, 197.6, 157.4, 136.1, 133.5, 131.8, 129.4, 128.7, 128.0, 121.7, 121.7, 111.1, 108.6, 65.2, 50.7, 45.7, 45.3, 36.4, 30.1, 21.0; FTIR (thin film) 2923, 1718, $1684,1474,1458,1448,1218,991,916,856,754,690 \mathrm{~cm}^{-1}$. Exact mass calculated for $\mathrm{C}_{22} \mathrm{H}_{20} \mathrm{O}_{3} \mathrm{~S}+\mathrm{Na}^{+}$(ES) 387.1033, found 387.1027.

Diketone 22', Diast. $2(4 \mathrm{mg}, 10 \%)$ as an oil: ${ }^{1} \mathrm{H}$ NMR $\left(500 \mathrm{MHz}, \mathrm{CDCl}_{3}\right) \delta 7.90(\mathrm{~d}, J=$ $8.2 \mathrm{~Hz}, 2 \mathrm{H}), 7.57$ (t, $J=7.6 \mathrm{~Hz}, 1 \mathrm{H}), 7.45(\mathrm{t}, J=7.2 \mathrm{~Hz}, 2 \mathrm{H}), 7.23(\mathrm{t}, J=7.6 \mathrm{~Hz}, 1 \mathrm{H})$, $7.14(\mathrm{~d}, J=7.5 \mathrm{~Hz}, 1 \mathrm{H}), 7.02(\mathrm{t}, J=7.5 \mathrm{~Hz}, 1 \mathrm{H}), 6.95(\mathrm{~d}, J=8.1 \mathrm{~Hz}, 1 \mathrm{H}), 3.84(\mathrm{~m}, 1 \mathrm{H})$, $3.35(\mathrm{dd}, J=17.3,6.0 \mathrm{~Hz}, 1 \mathrm{H}), 3.23(\mathrm{dd}, J=17.3,7.8 \mathrm{~Hz}, 1 \mathrm{H}), 3.02(\mathrm{dd}, J=12.8,4.4$ $\mathrm{Hz}, 1 \mathrm{H}), 2.66(\mathrm{~m}, 2 \mathrm{H}), 2.12(\mathrm{t}, J=11.7 \mathrm{~Hz}, 1 \mathrm{H}), 1.95(\mathrm{~m}, 2 \mathrm{H}), 1.87(\mathrm{t}, J=10.7 \mathrm{~Hz}, 1 \mathrm{H})$, $1.76(\mathrm{~m}, 1 \mathrm{H}) ;{ }^{13} \mathrm{C}$ NMR $\left(125 \mathrm{MHz}, \mathrm{CDCl}_{3}\right) \delta$ 201.9, 197.0, 157.9, 136.3, 133.5, 131.9, 129.4, 128.7, 128.0, 122.7, 122.4, 111.1, 109.6, 64.0, 50.2, 43.0, 42.8, 35.9, 34.8, 18.5; FTIR (thin film) 2939, 1718, 1685, 1596, 1476, 1458, 1448, 1219, 913, $742 \mathrm{~cm}^{-1}$. Exact mass calculated for $\mathrm{C}_{22} \mathrm{H}_{20} \mathrm{O}_{3} \mathrm{~S}+\mathrm{Na}^{+}$(ES) 387.1033, found 387.1019. 


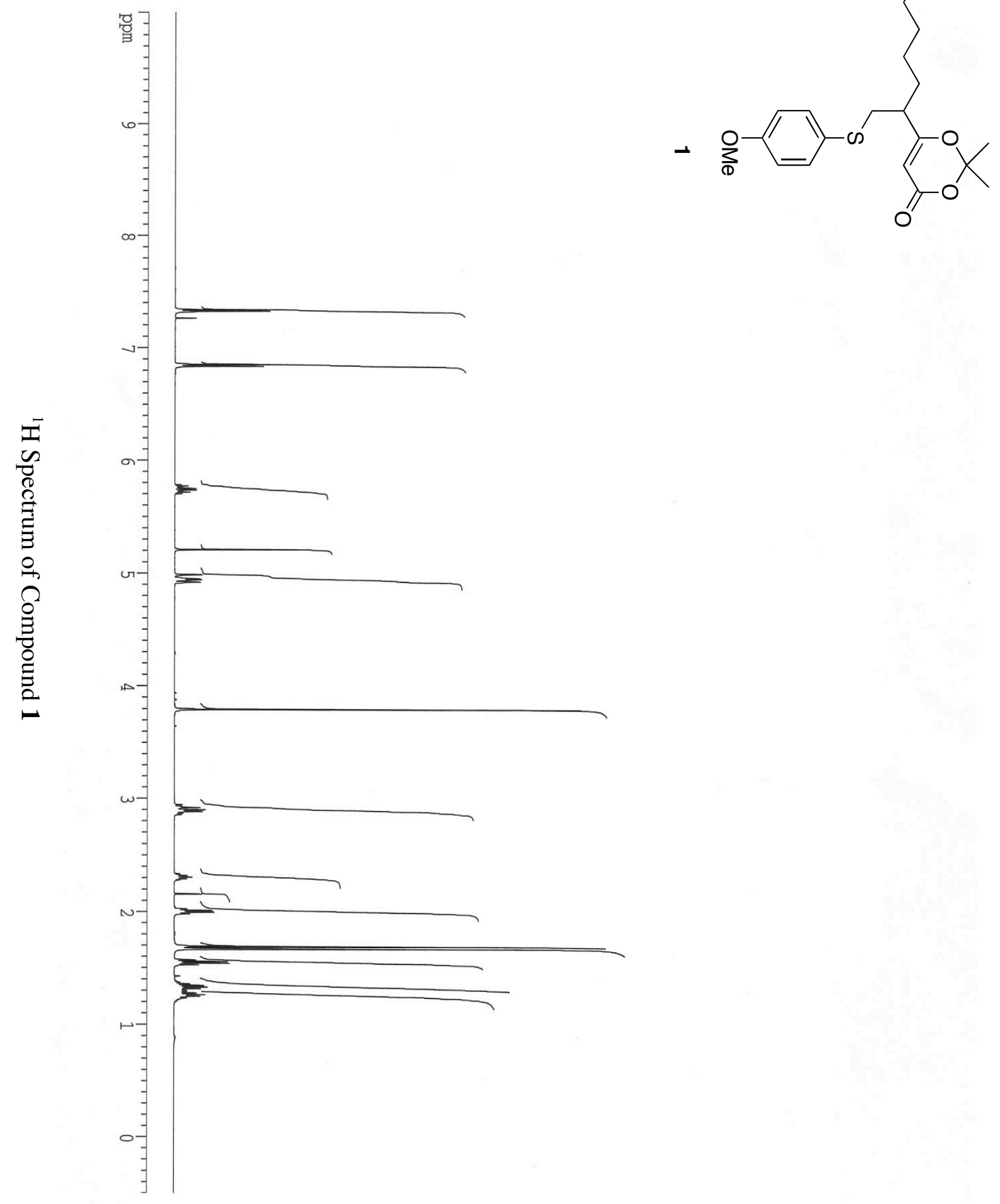




$$
k^{*}
$$




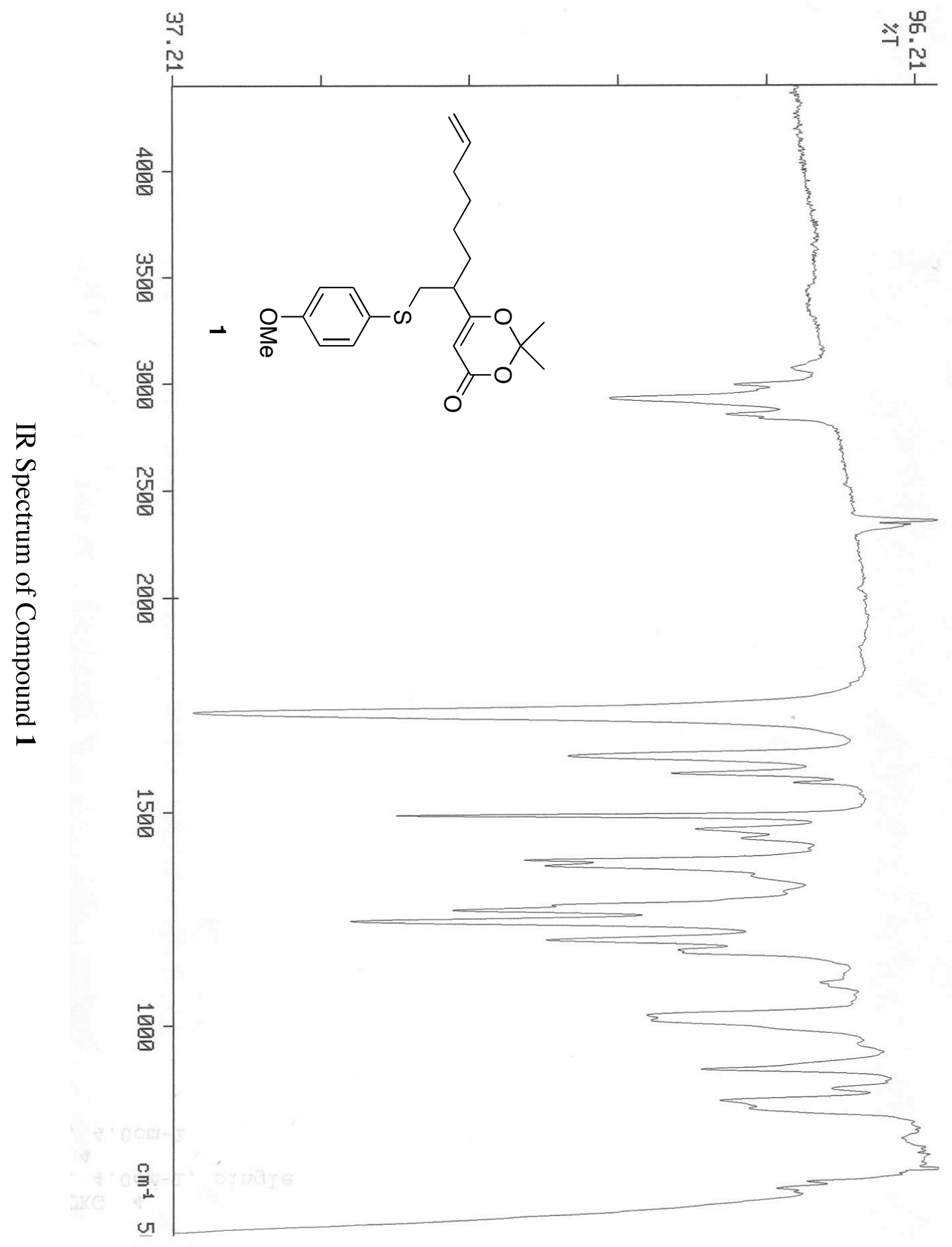




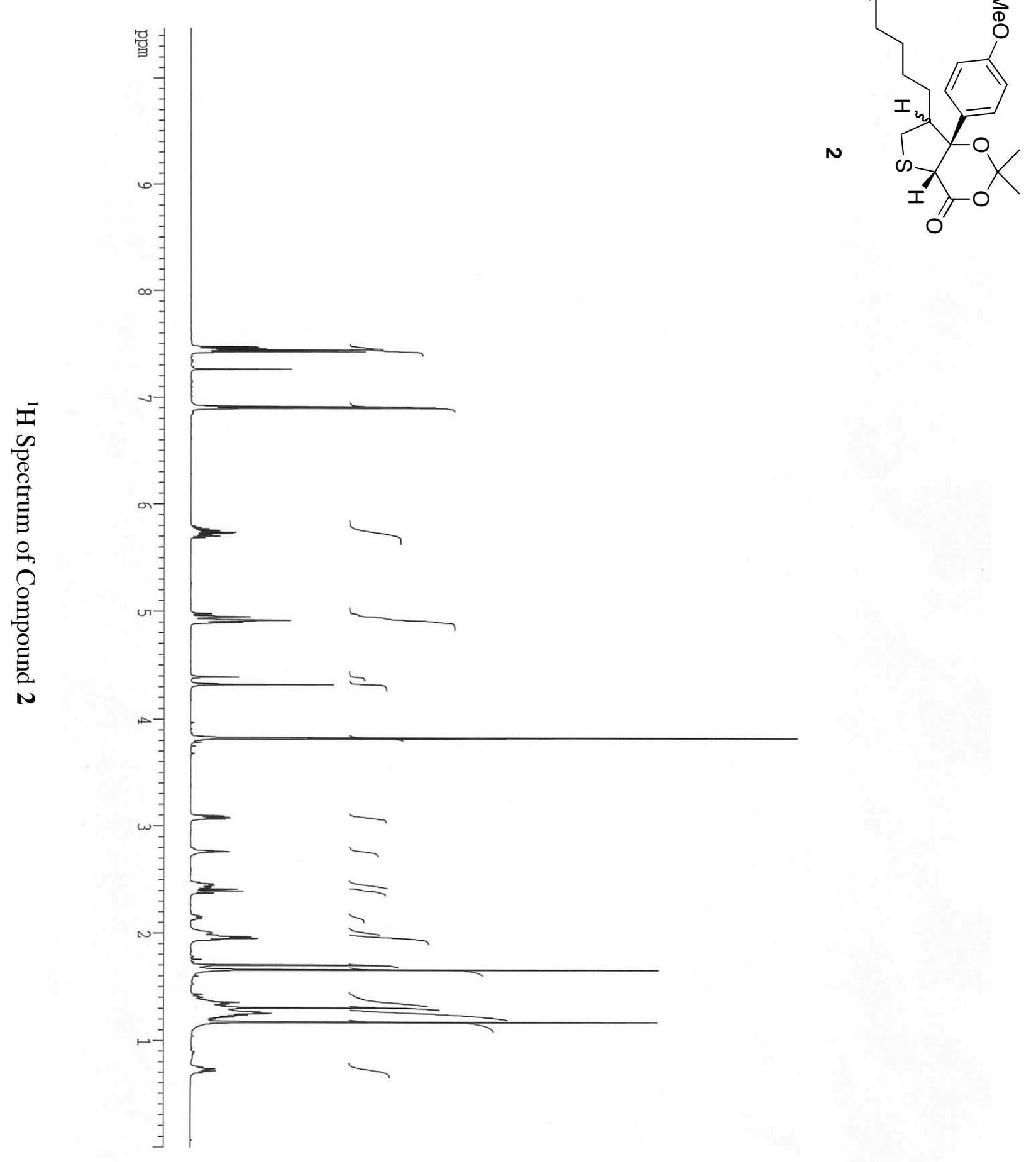




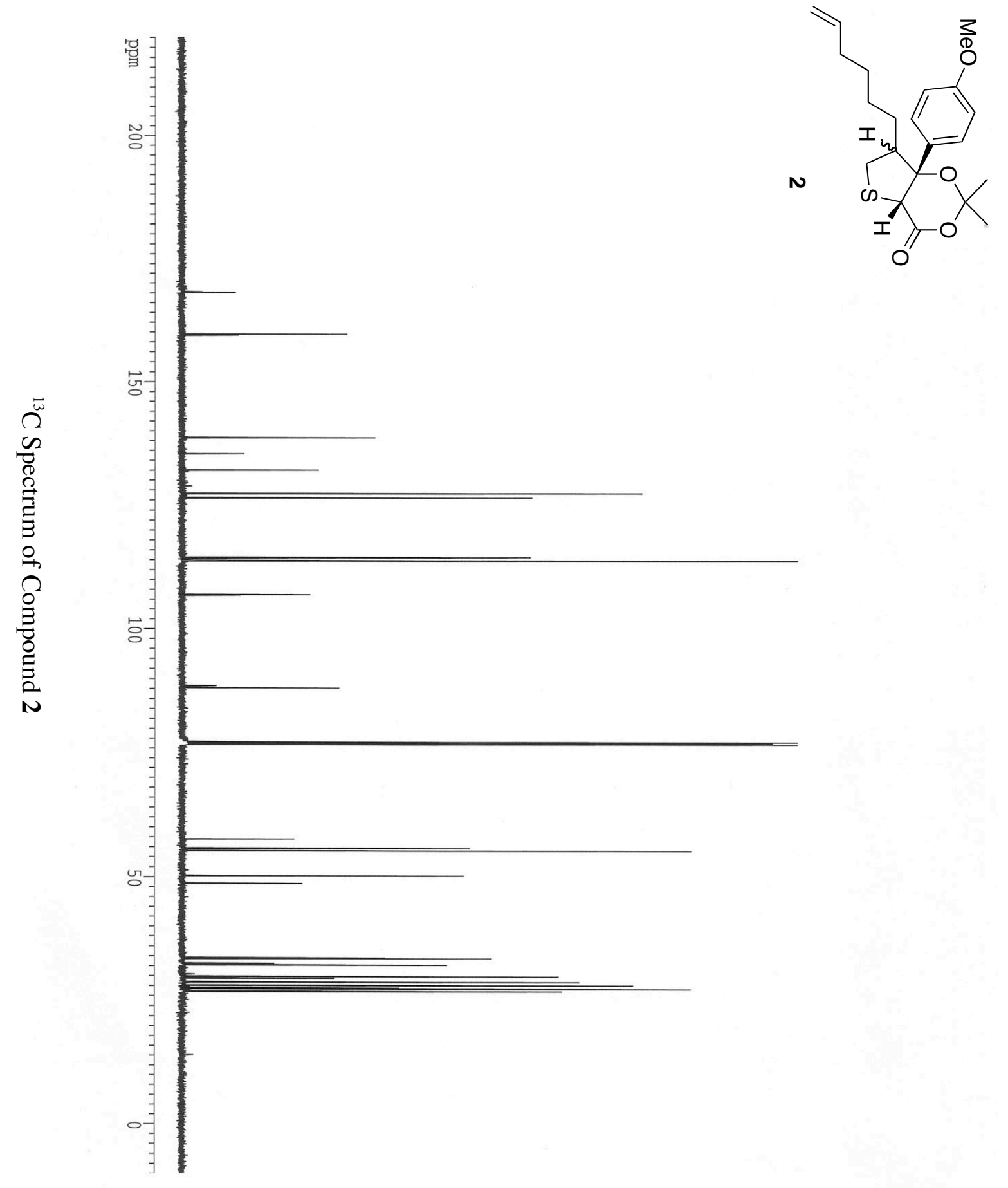




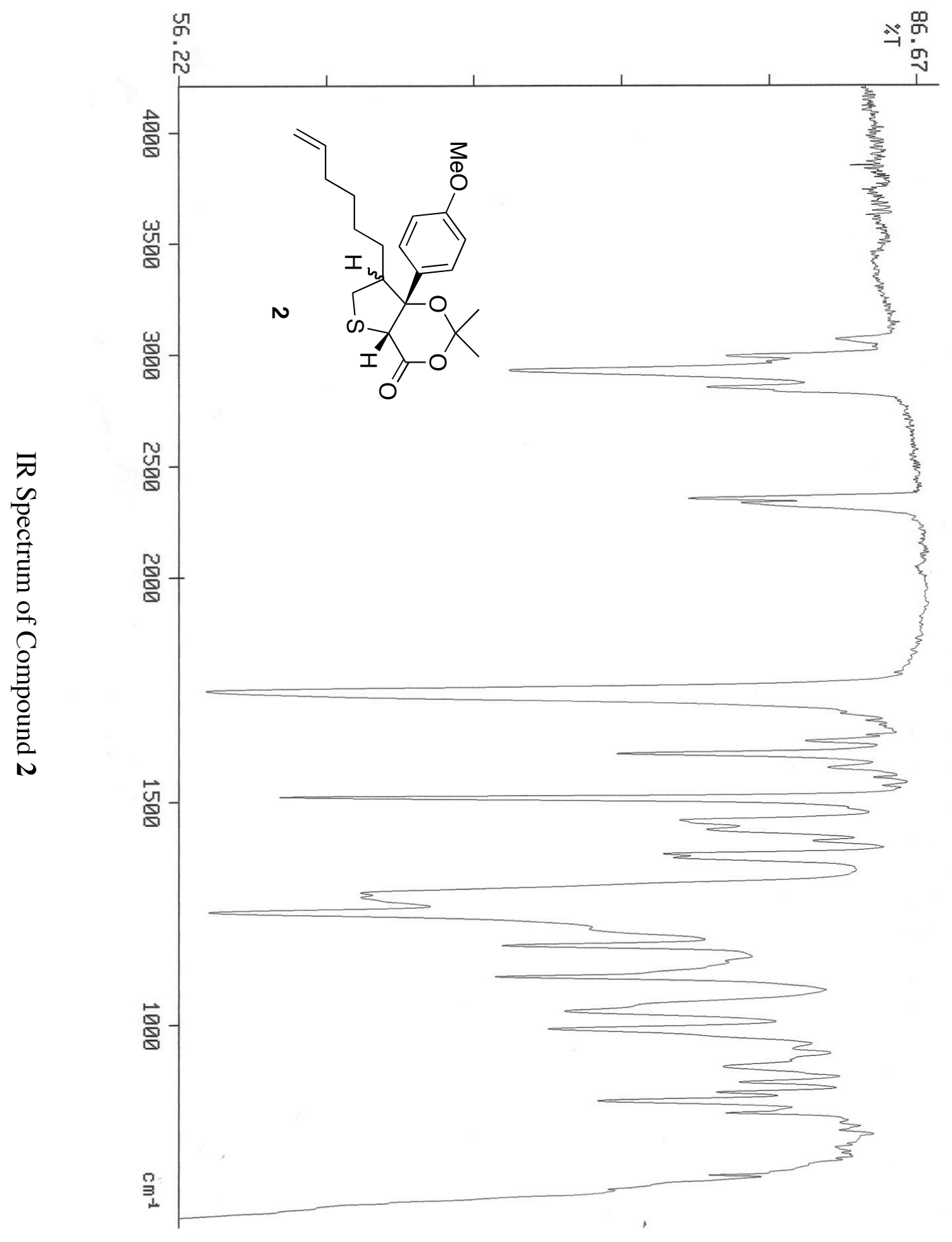

S-25 

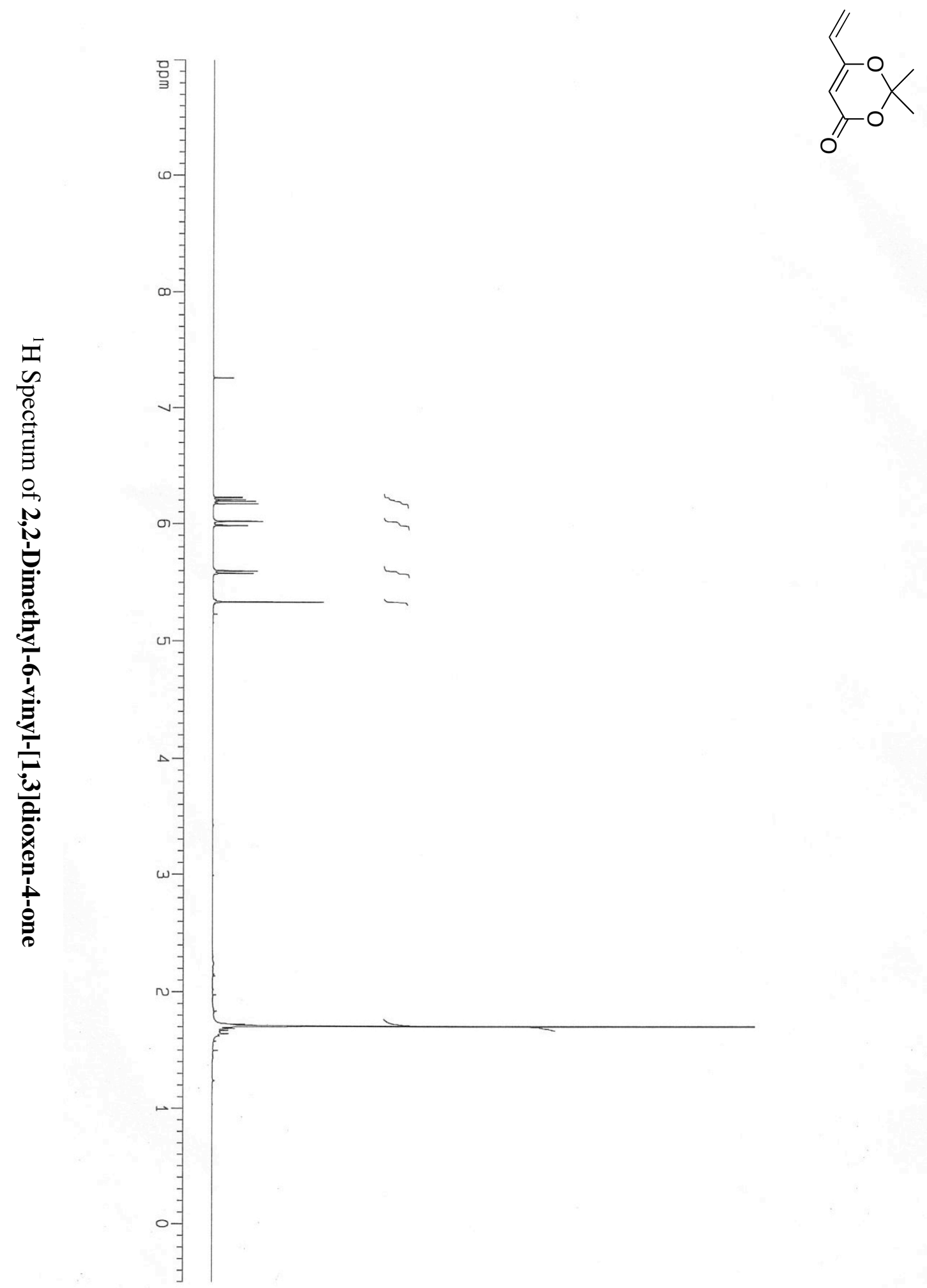

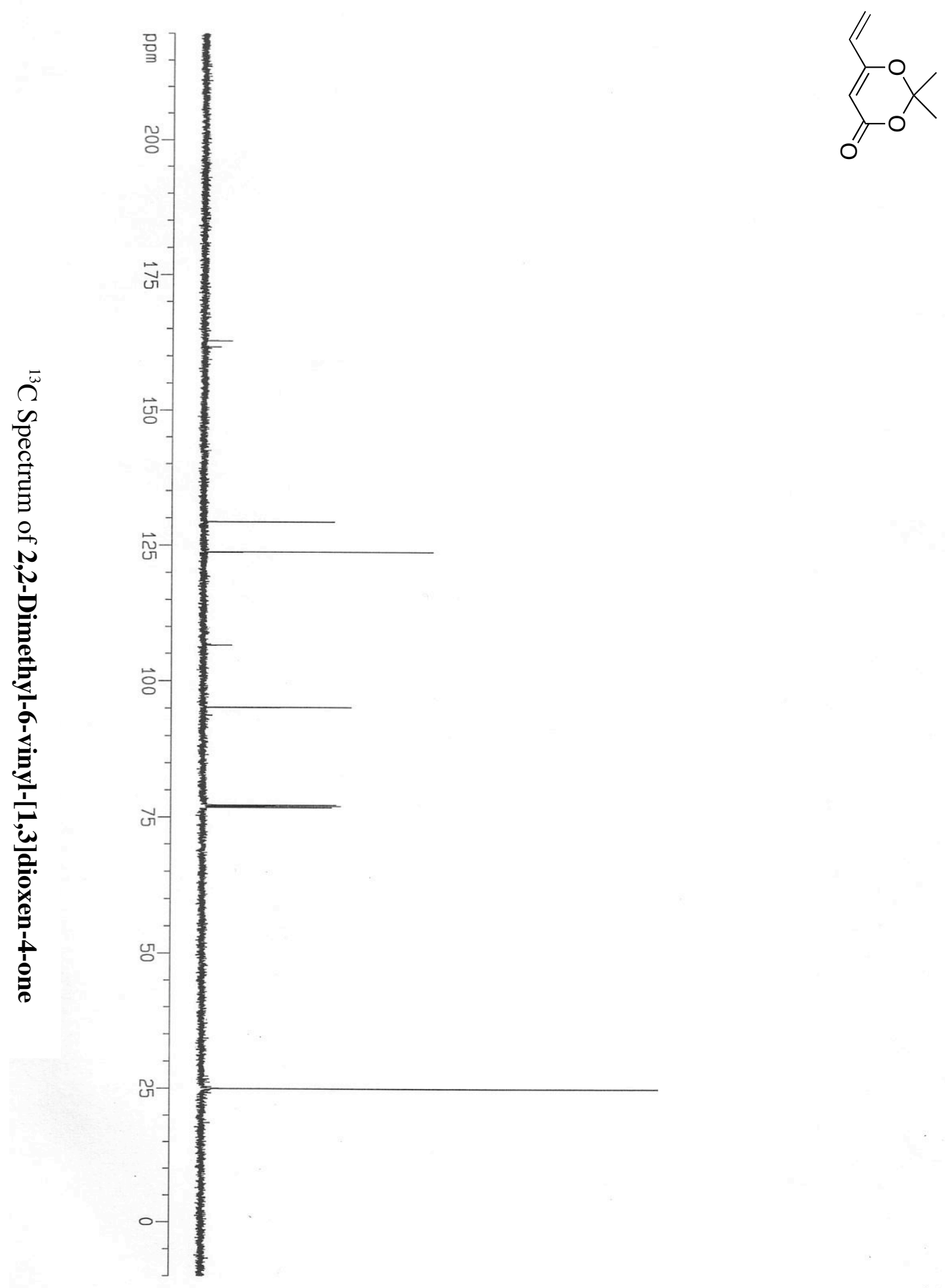


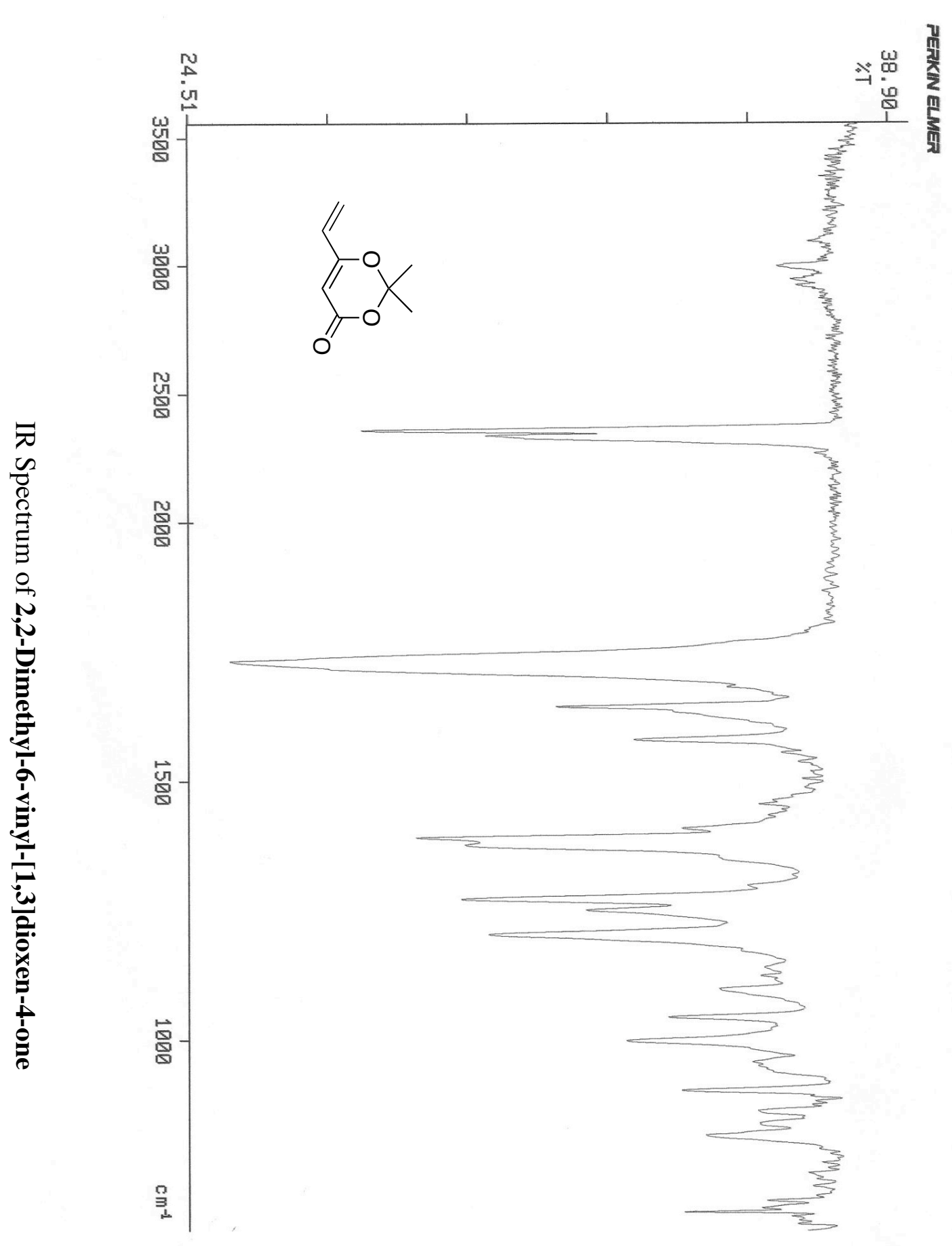



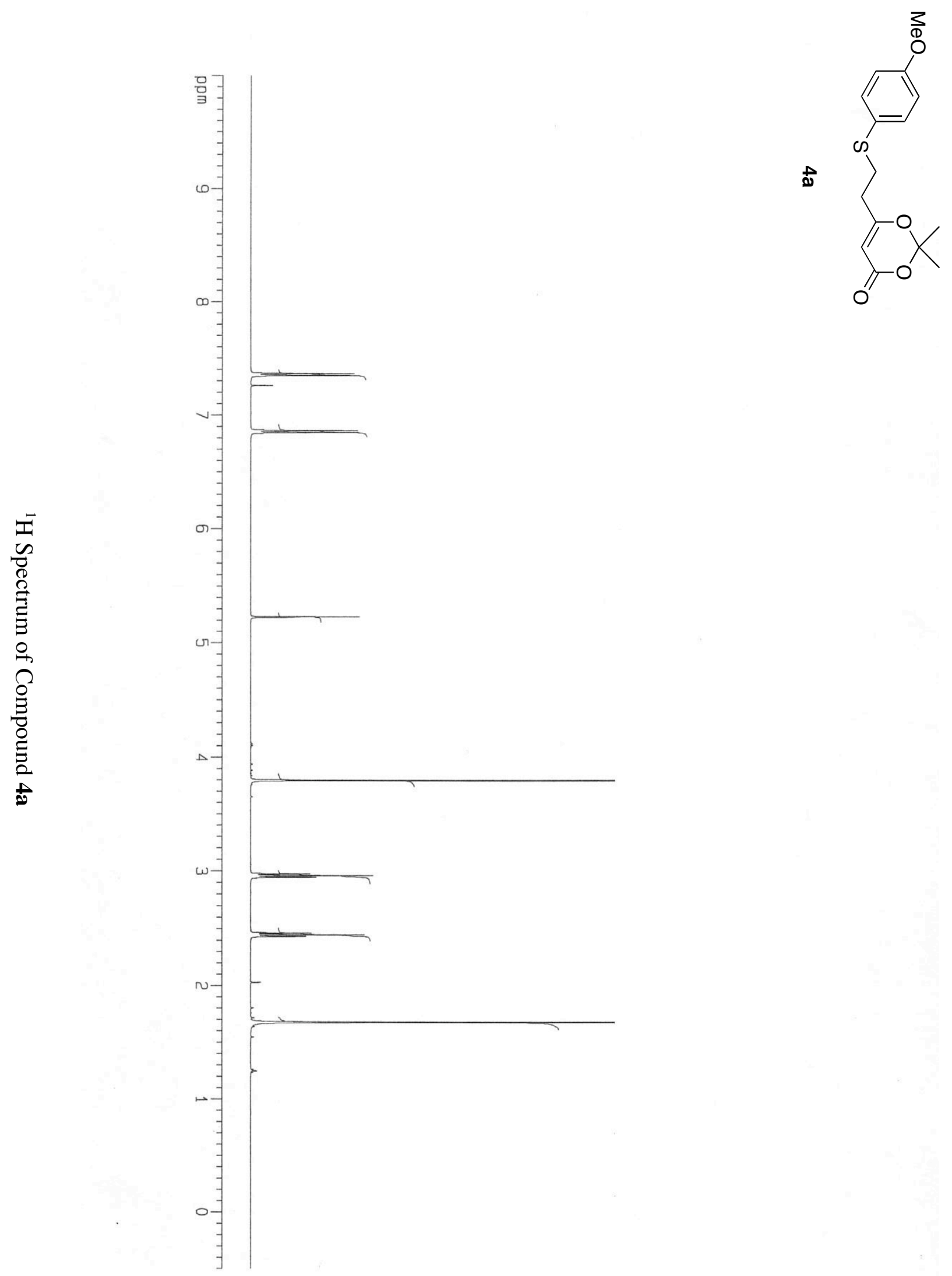

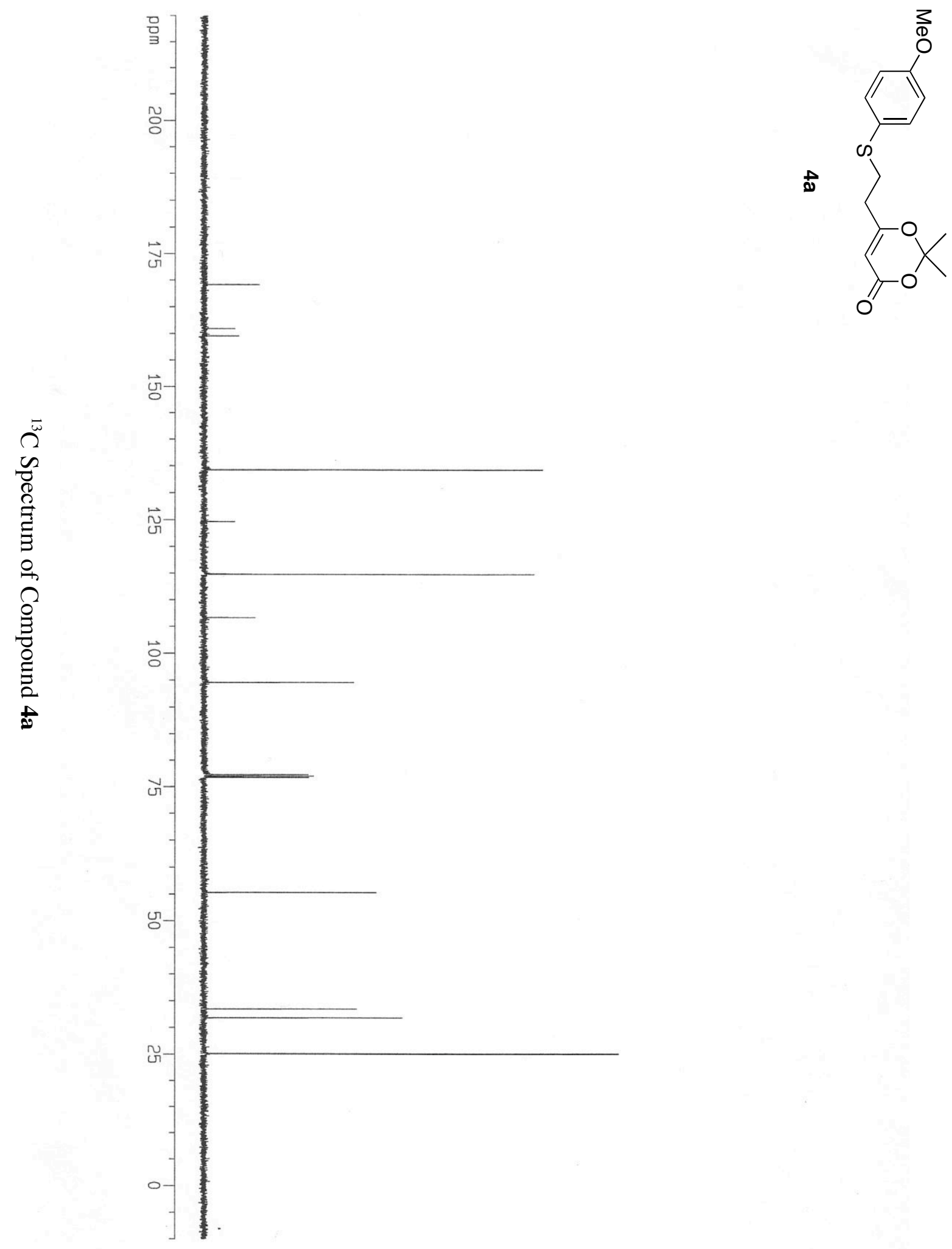


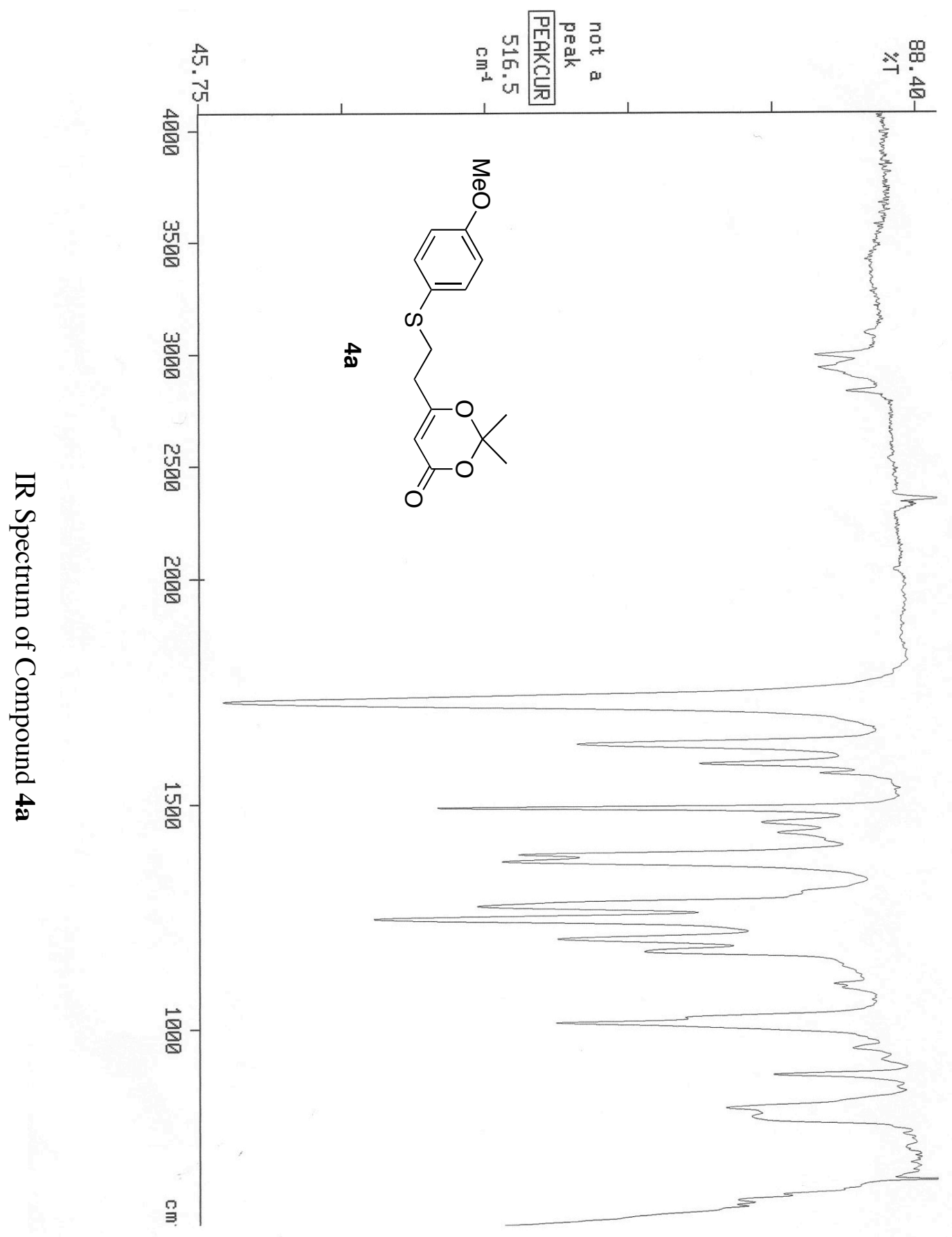

S-31 

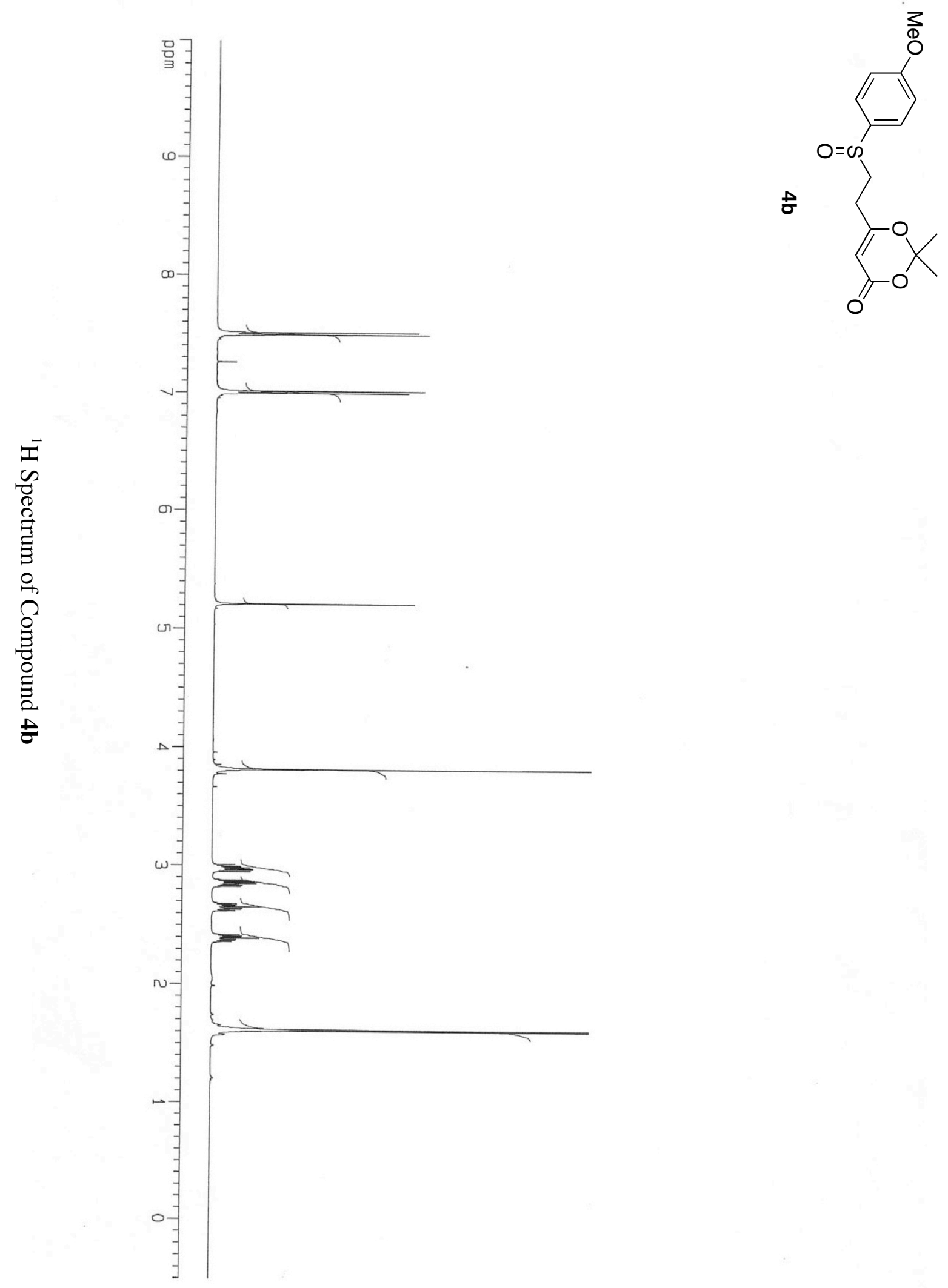

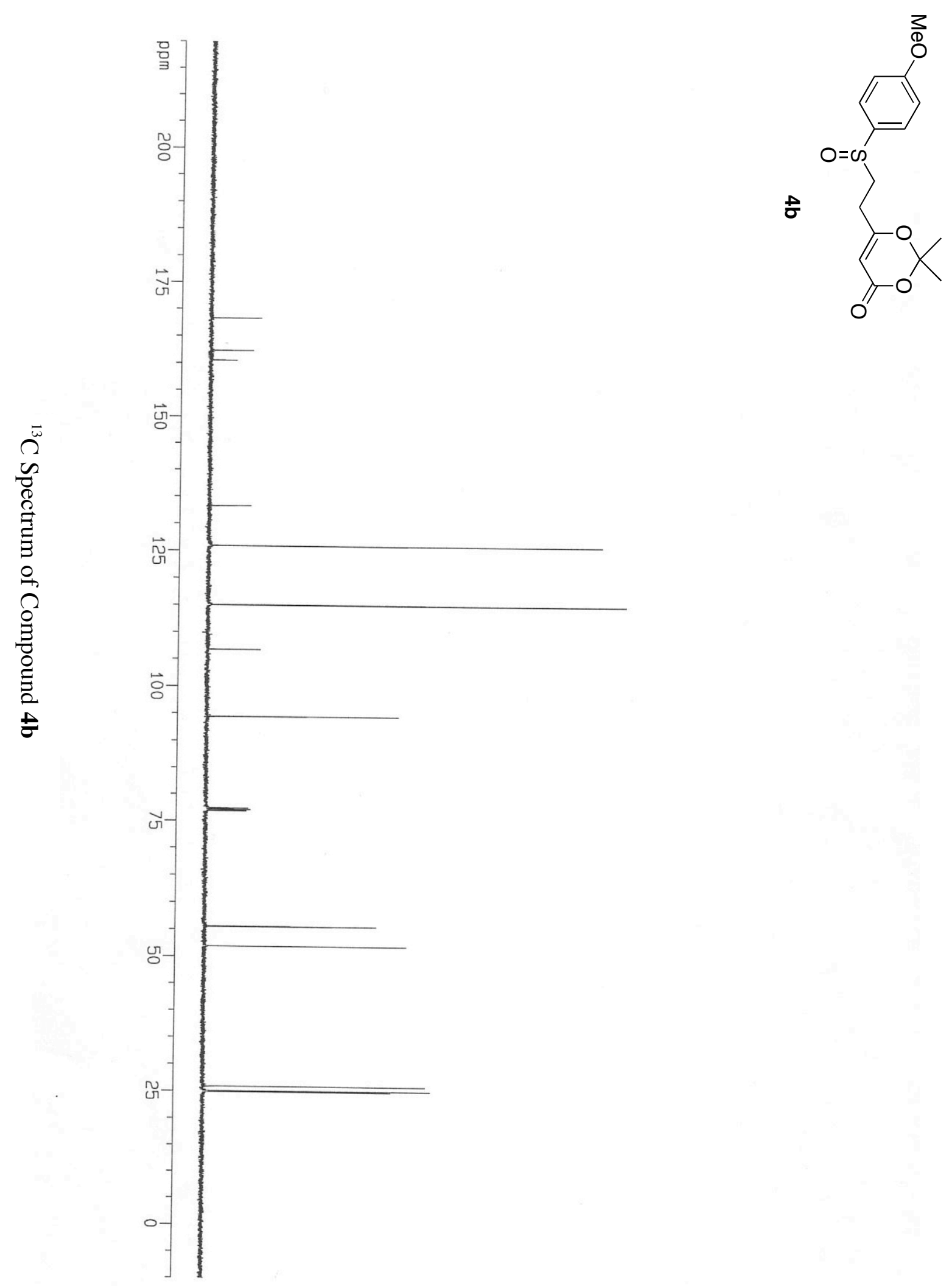

S-33 


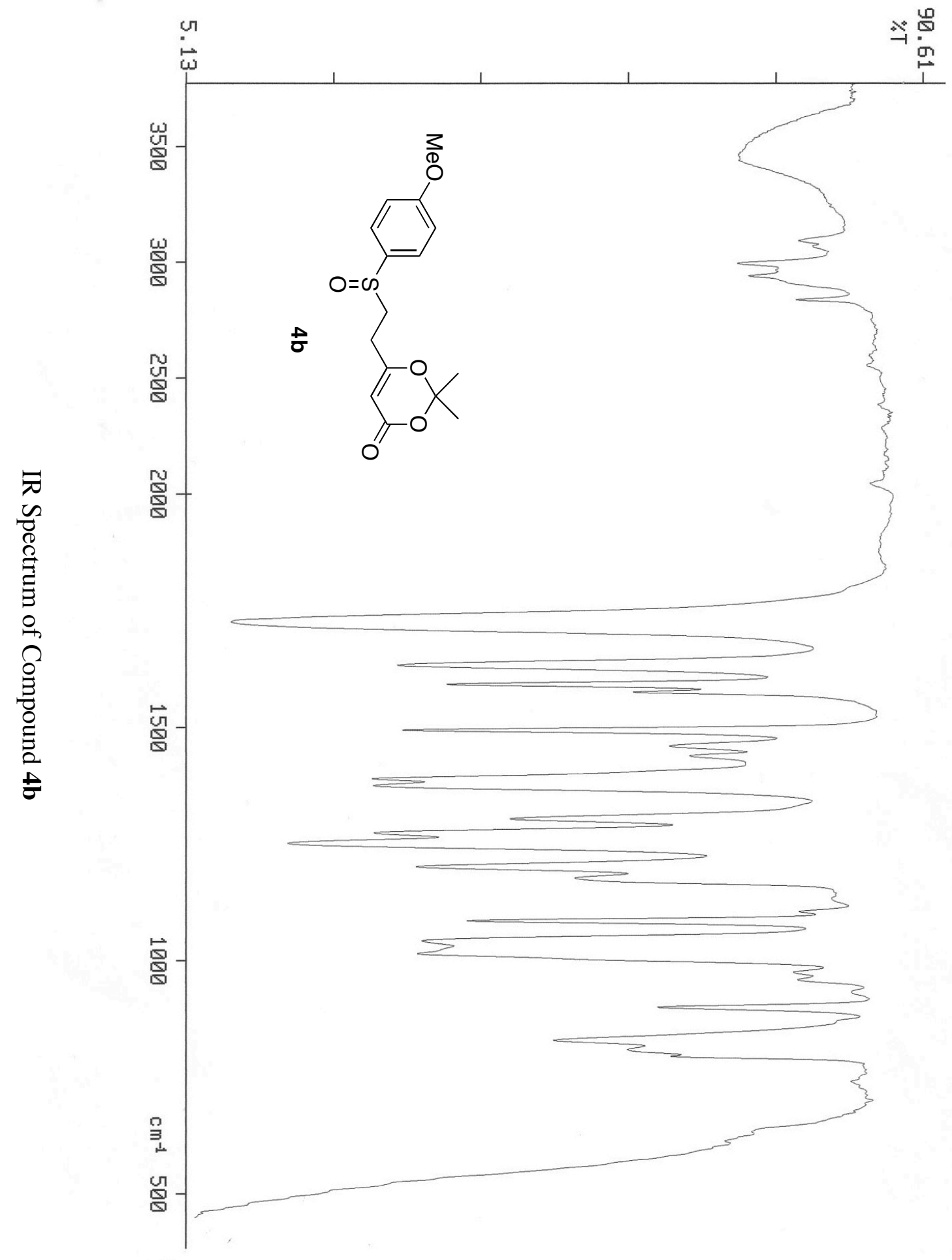



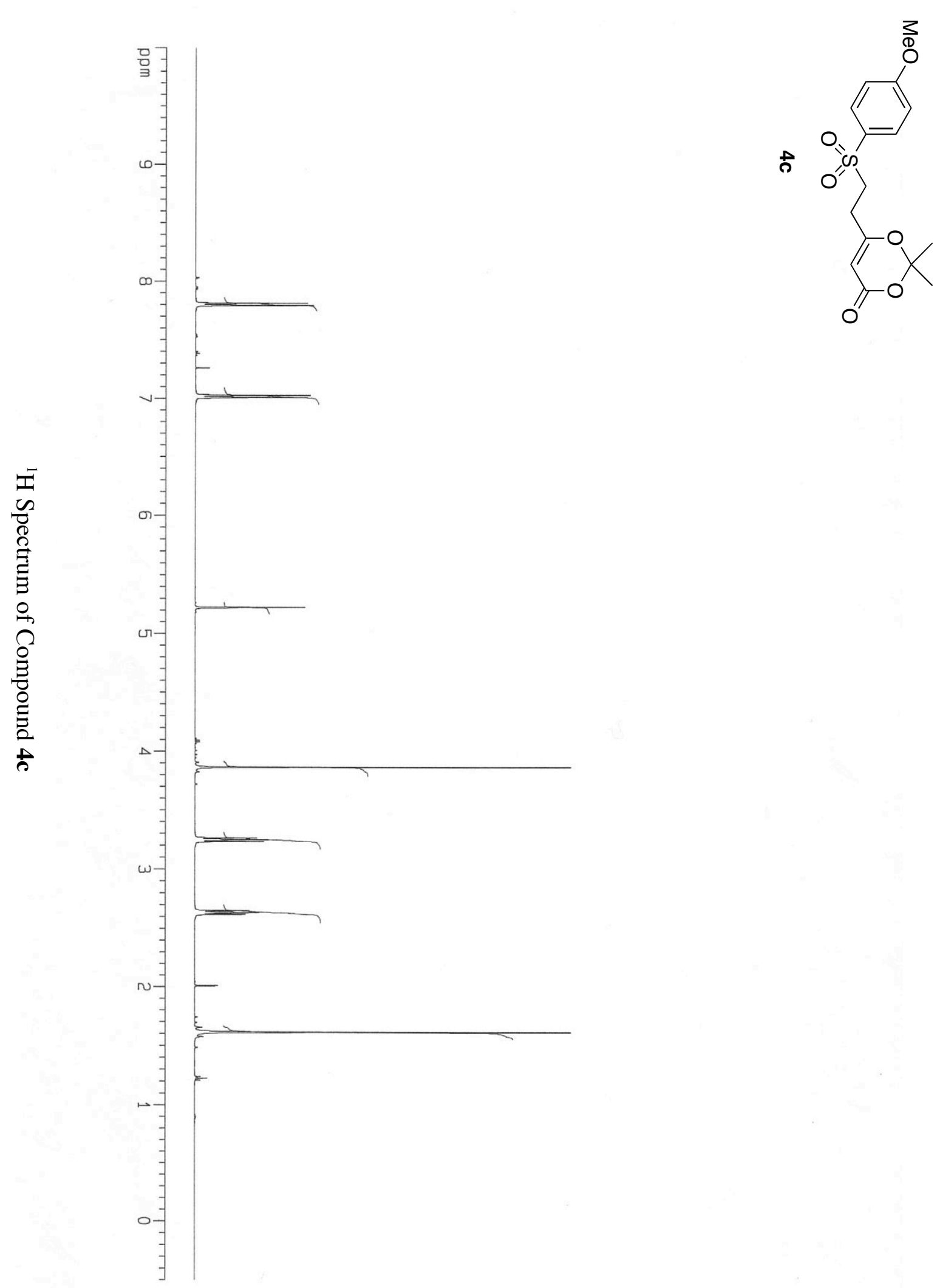

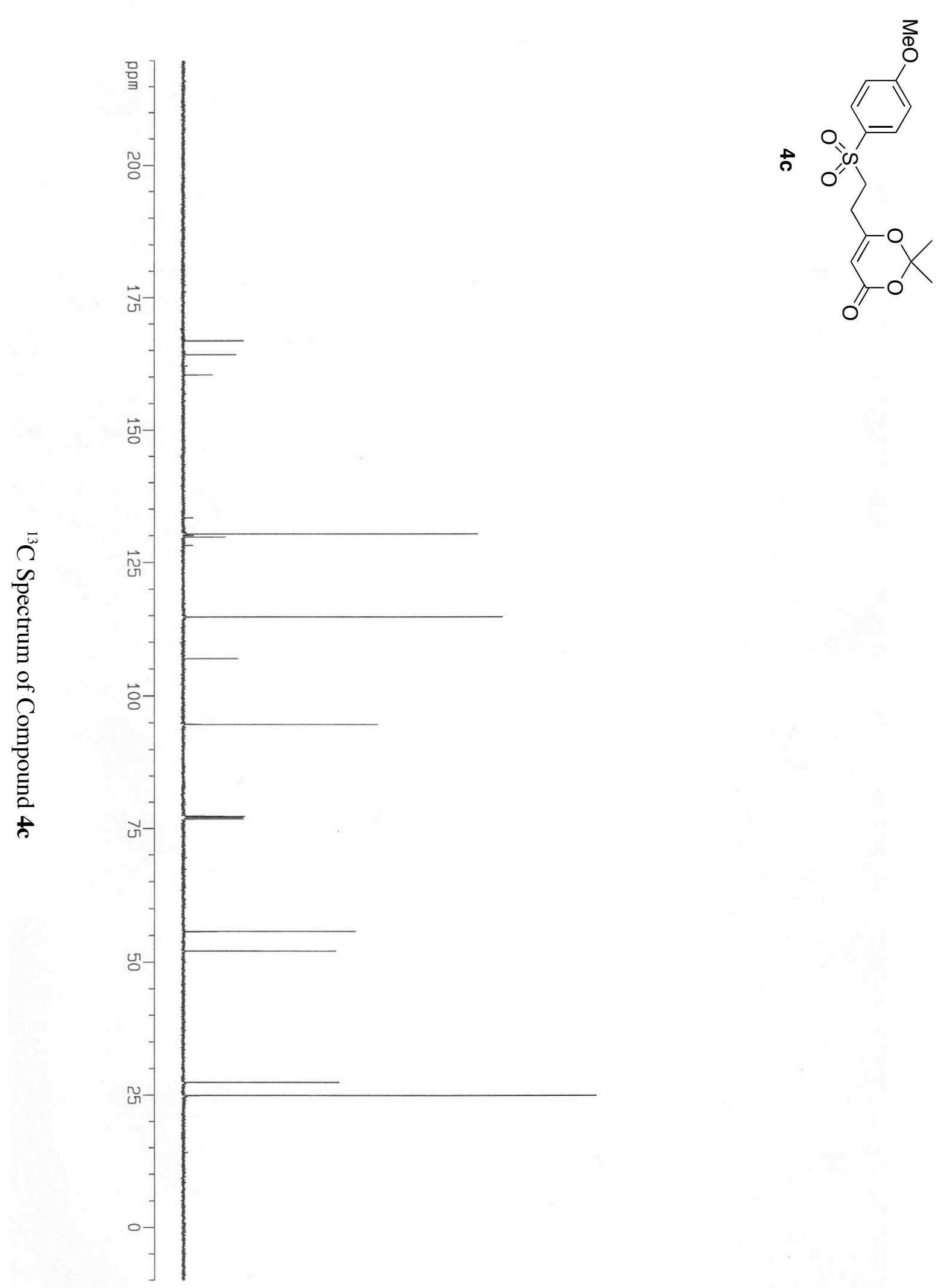


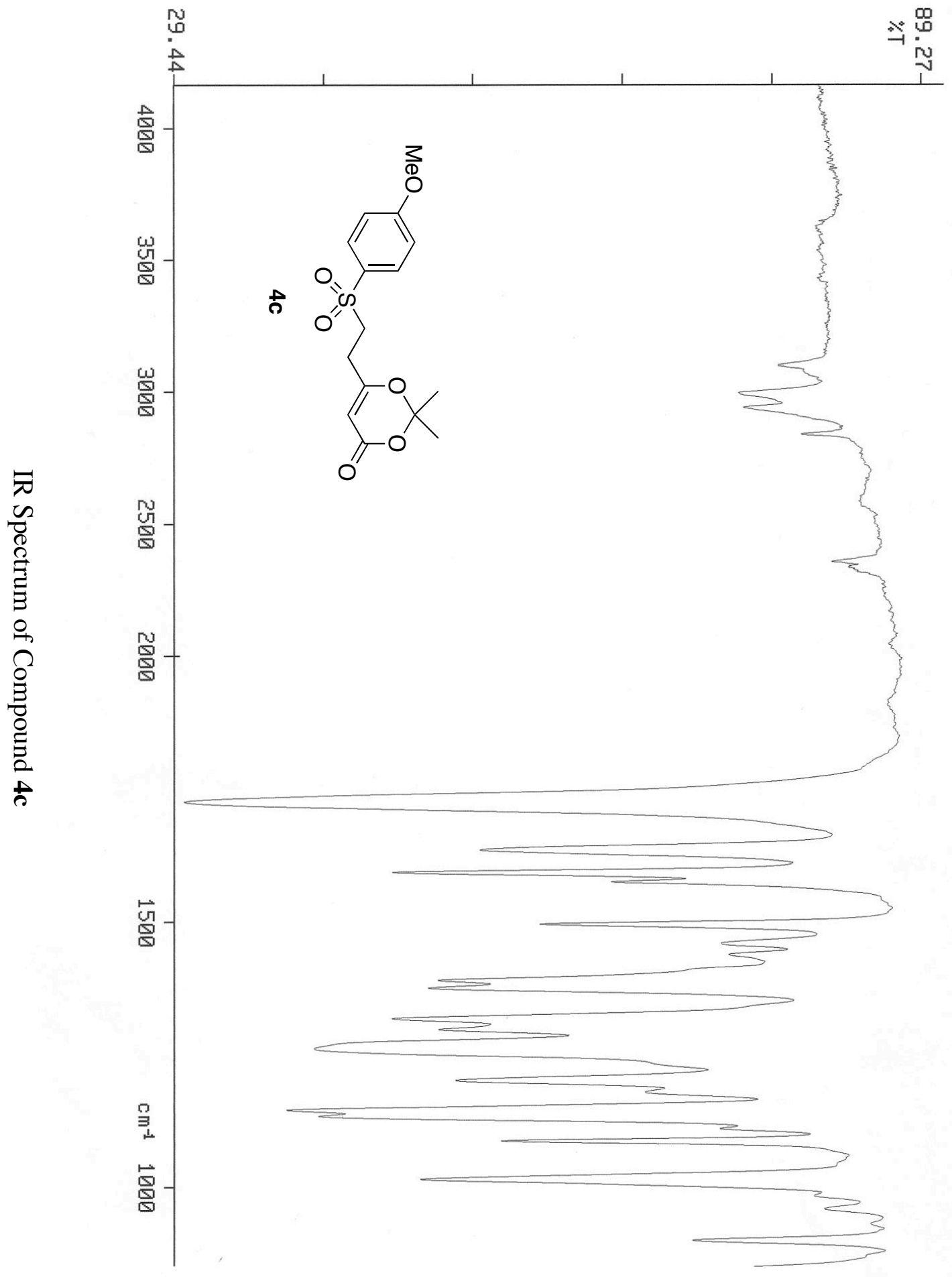



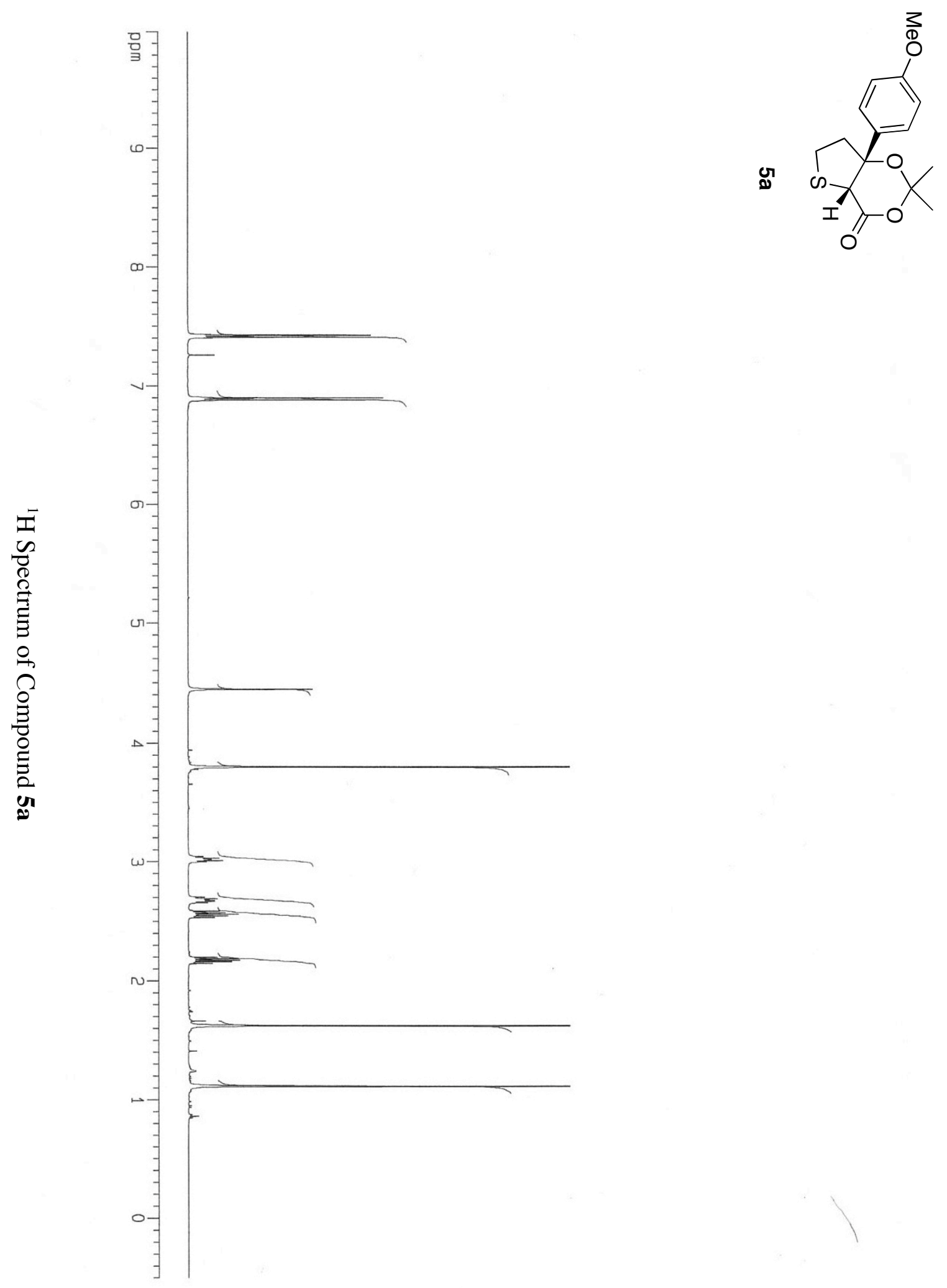

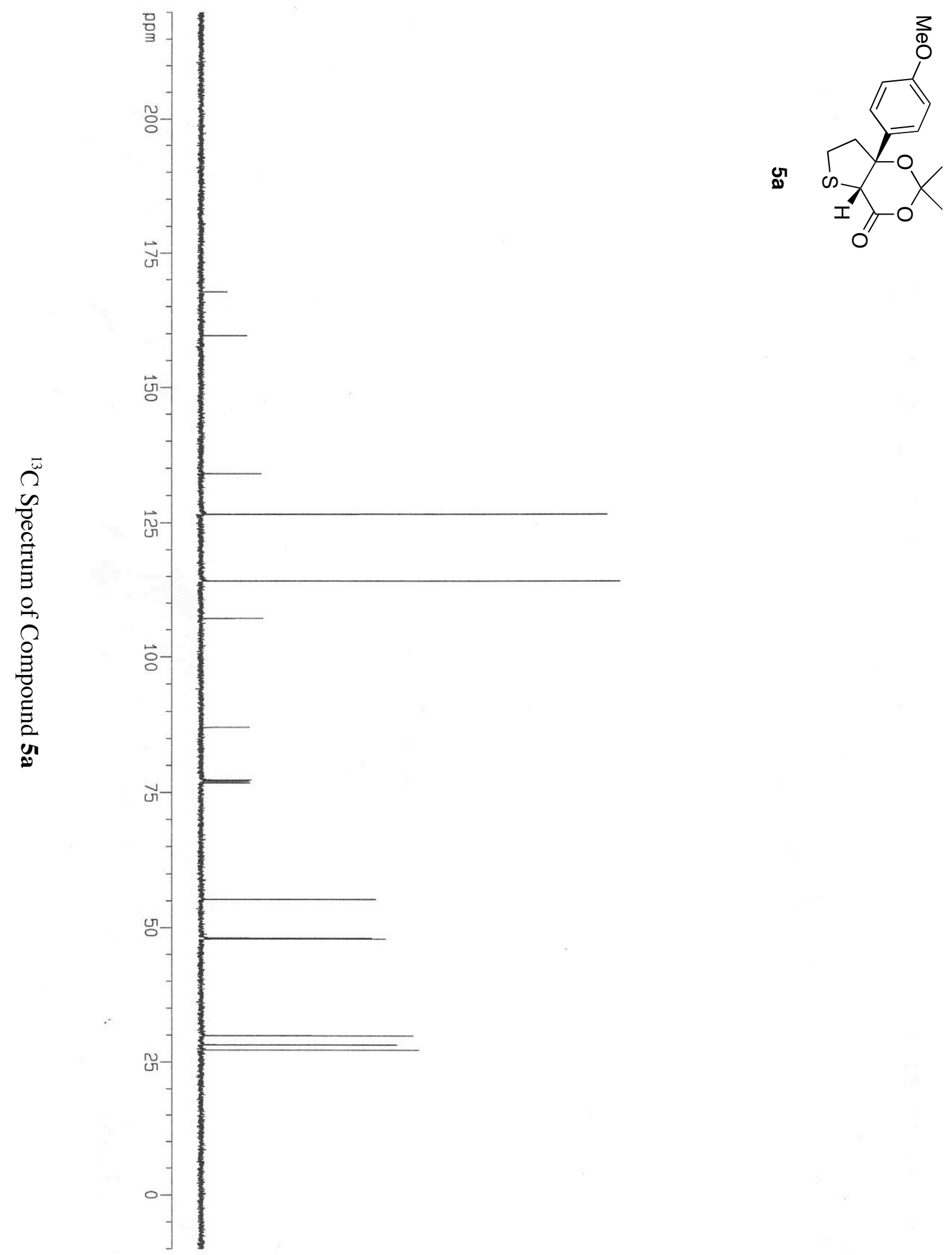


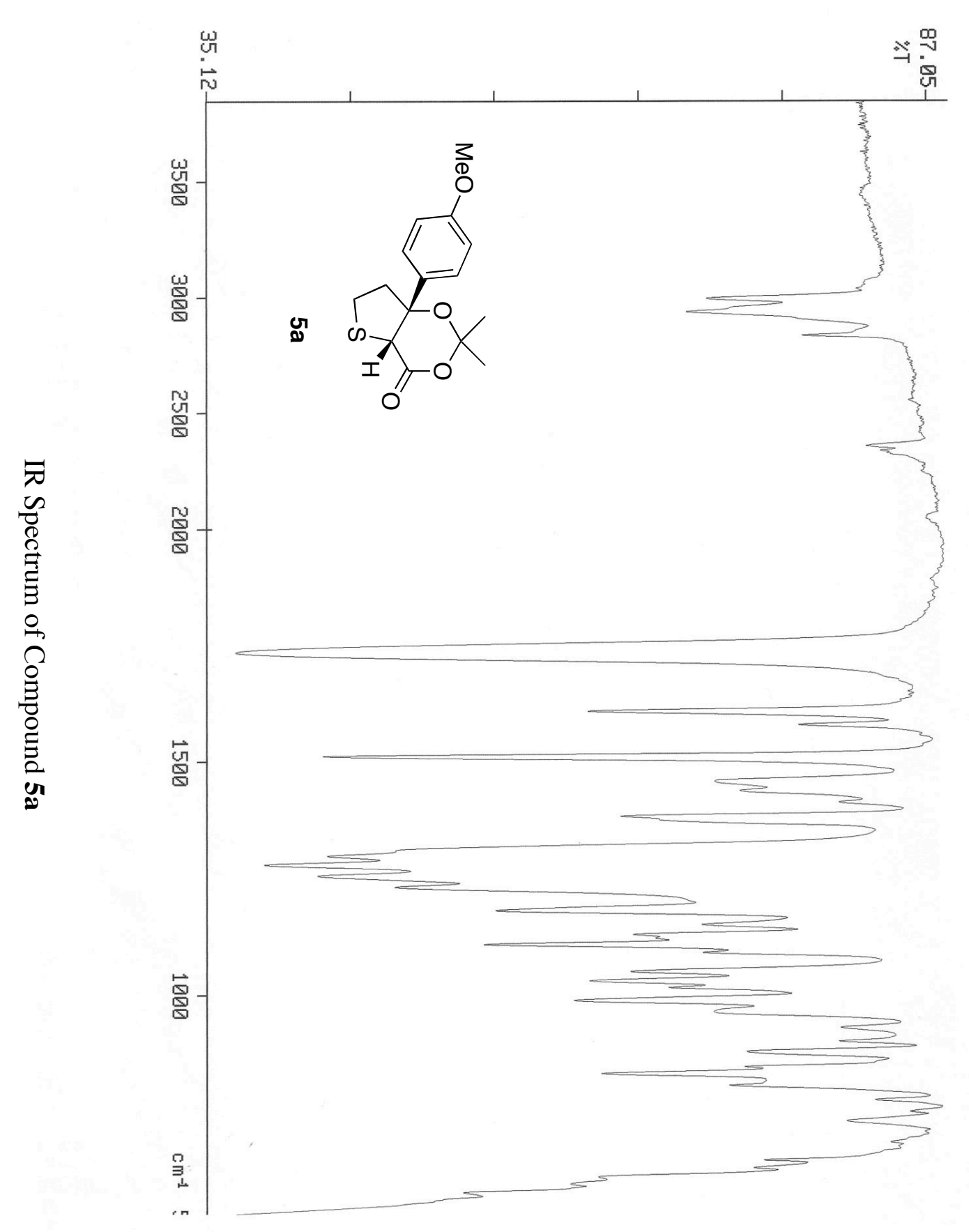



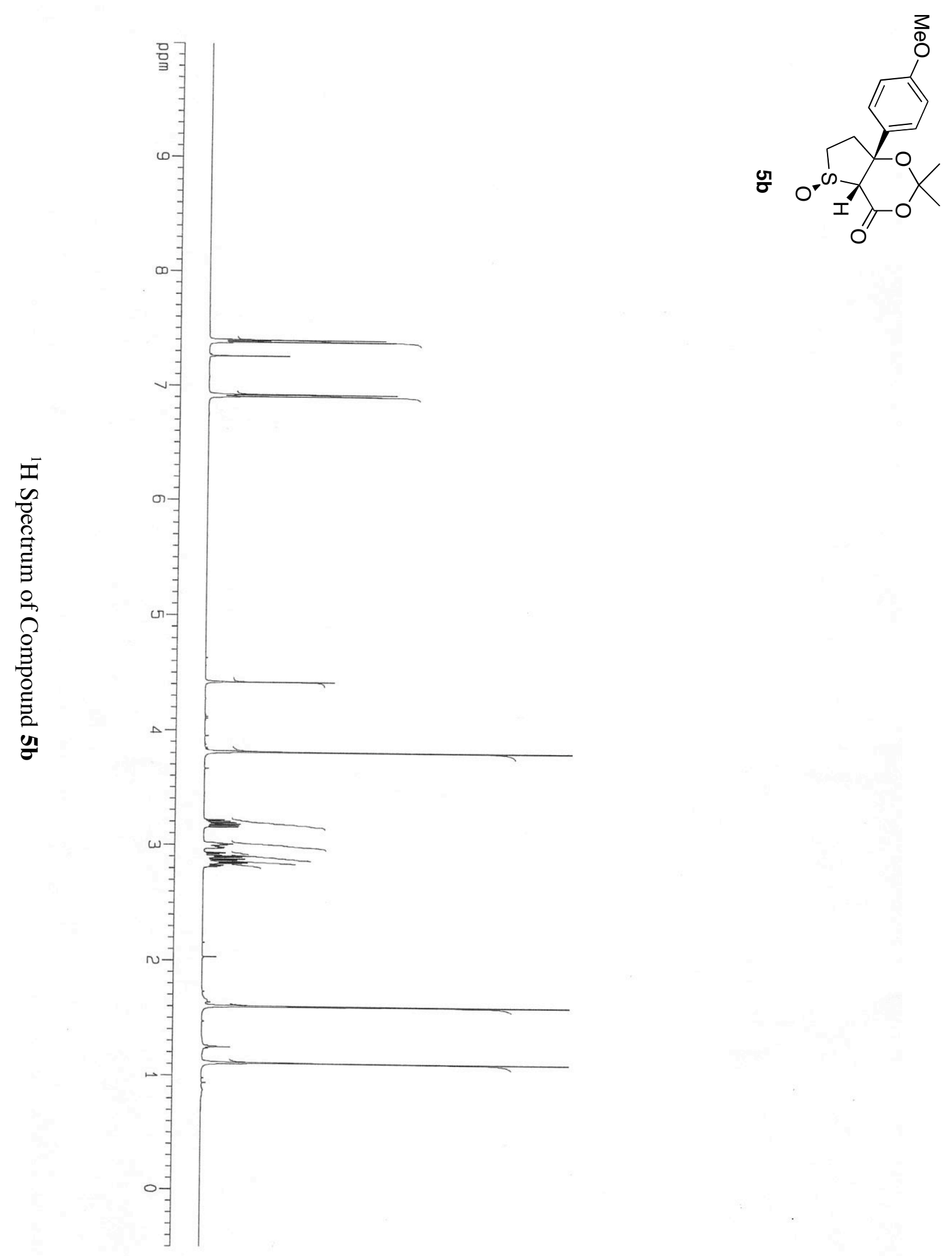

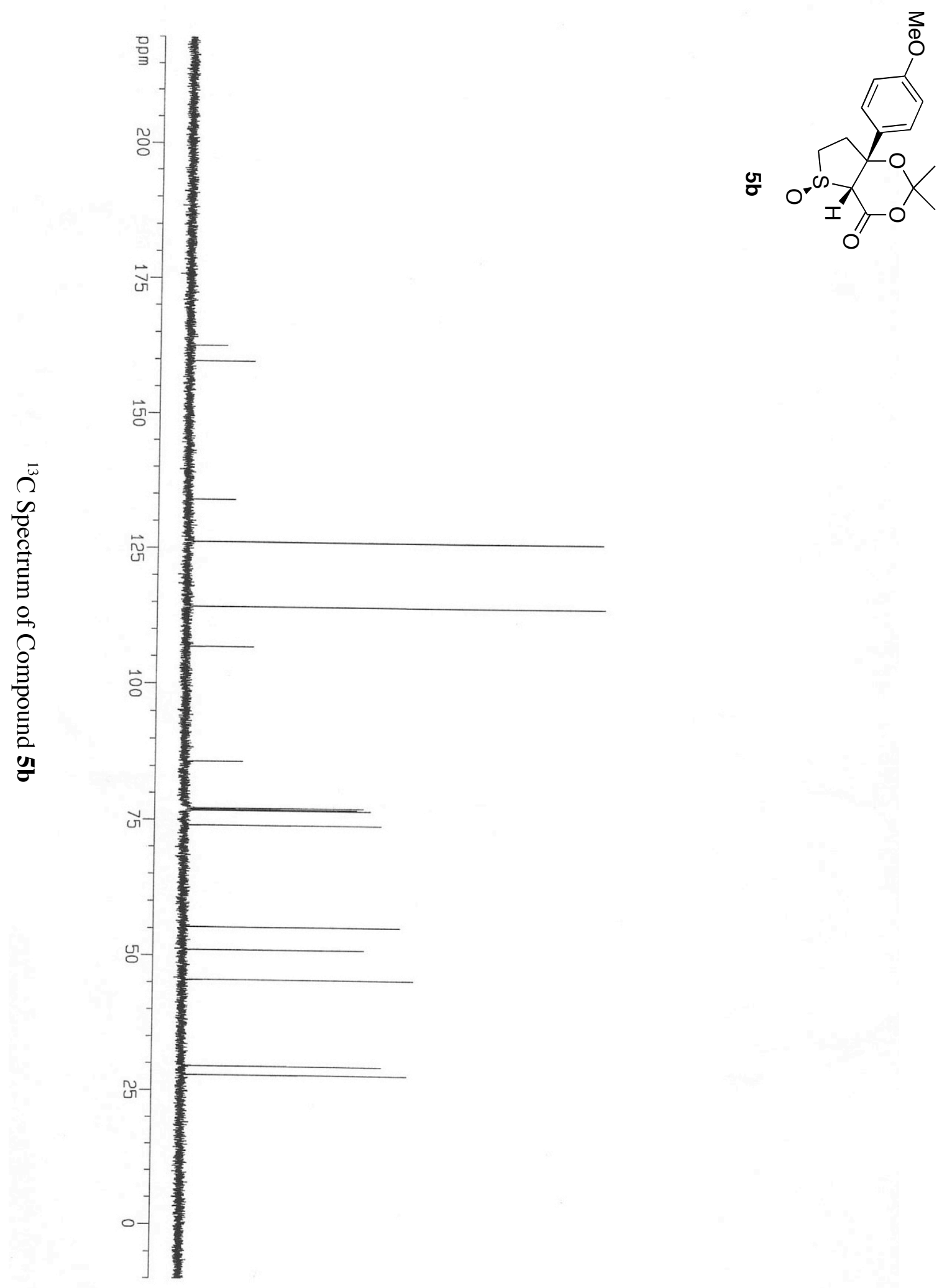


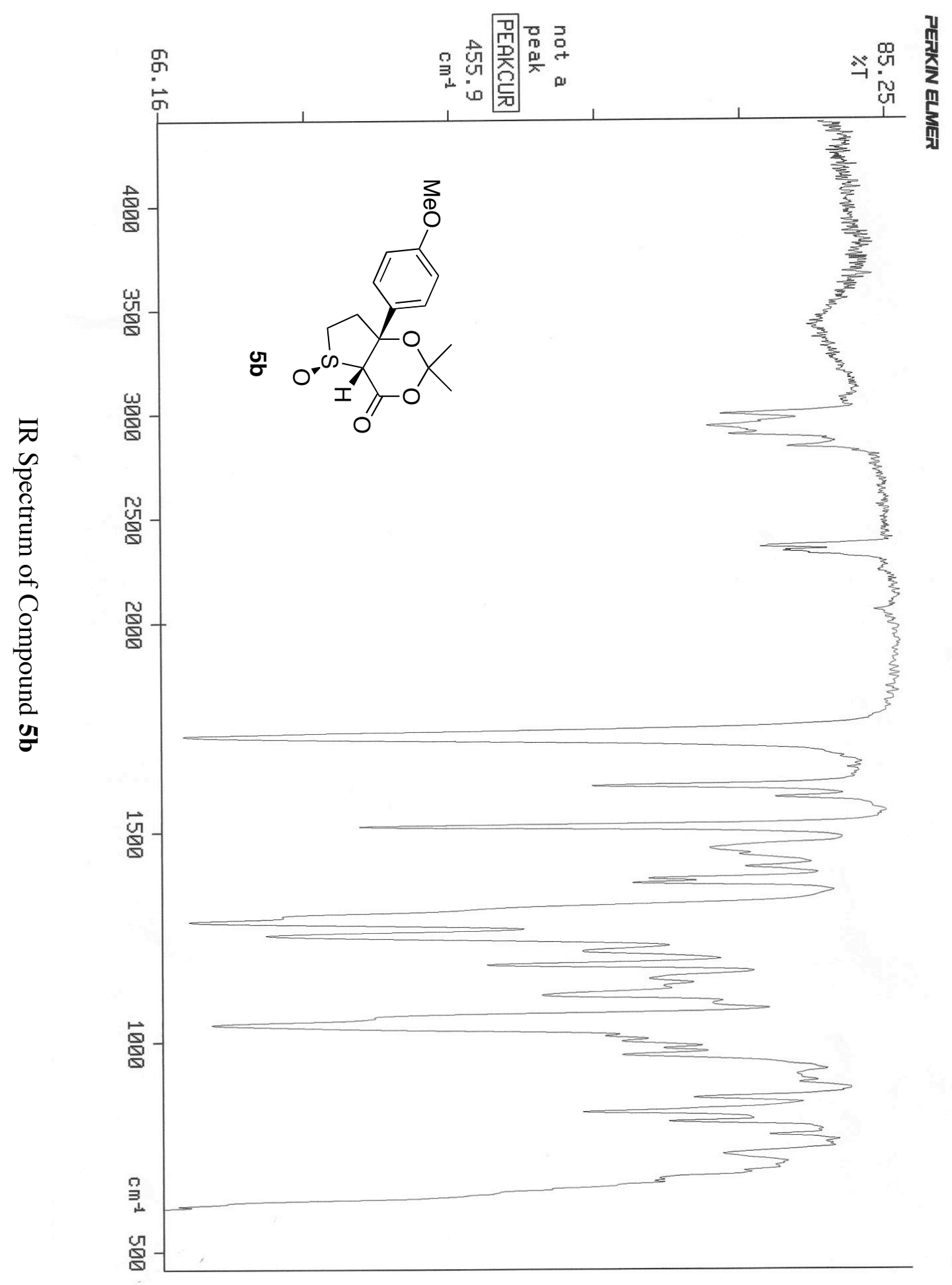



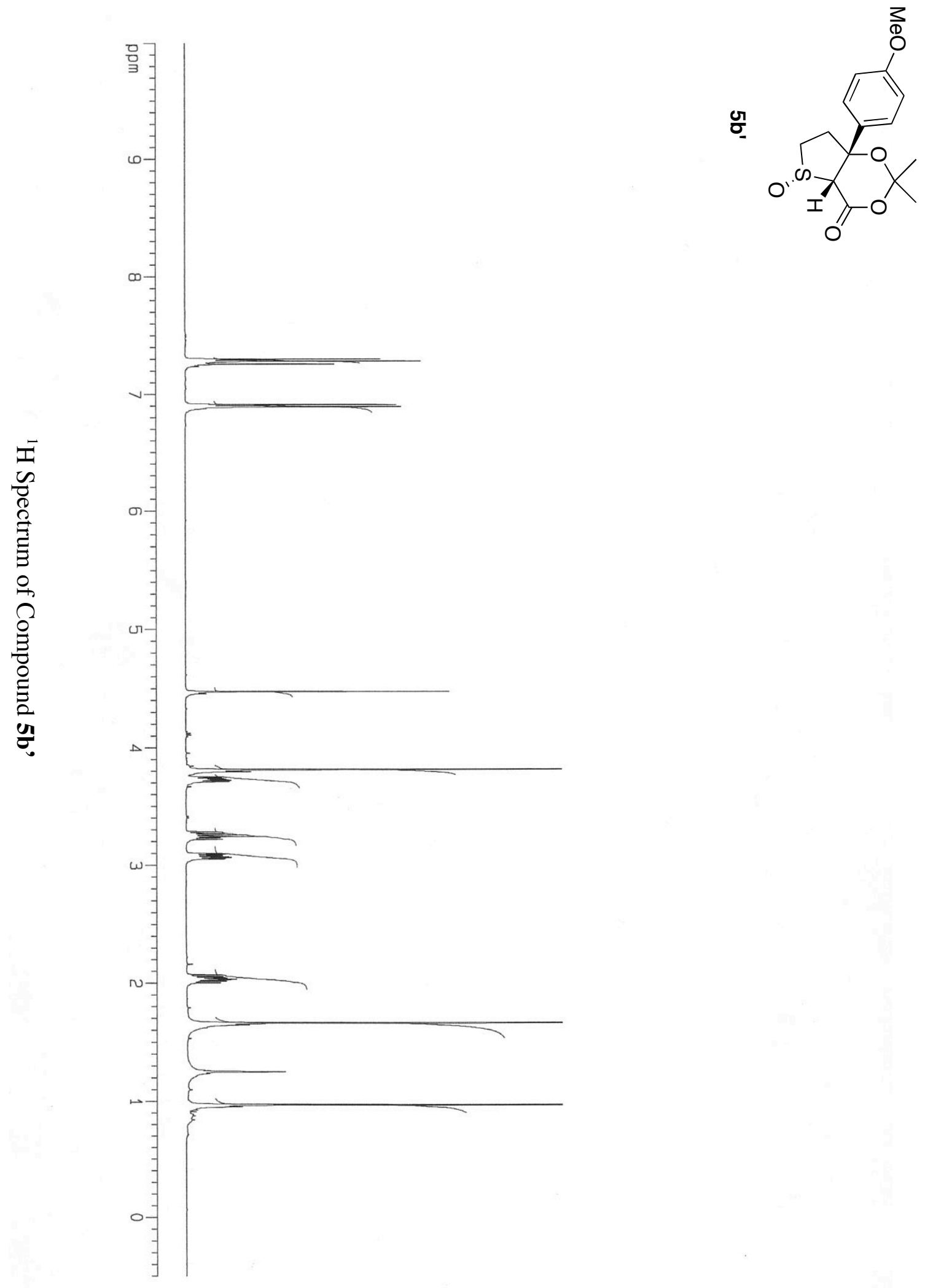

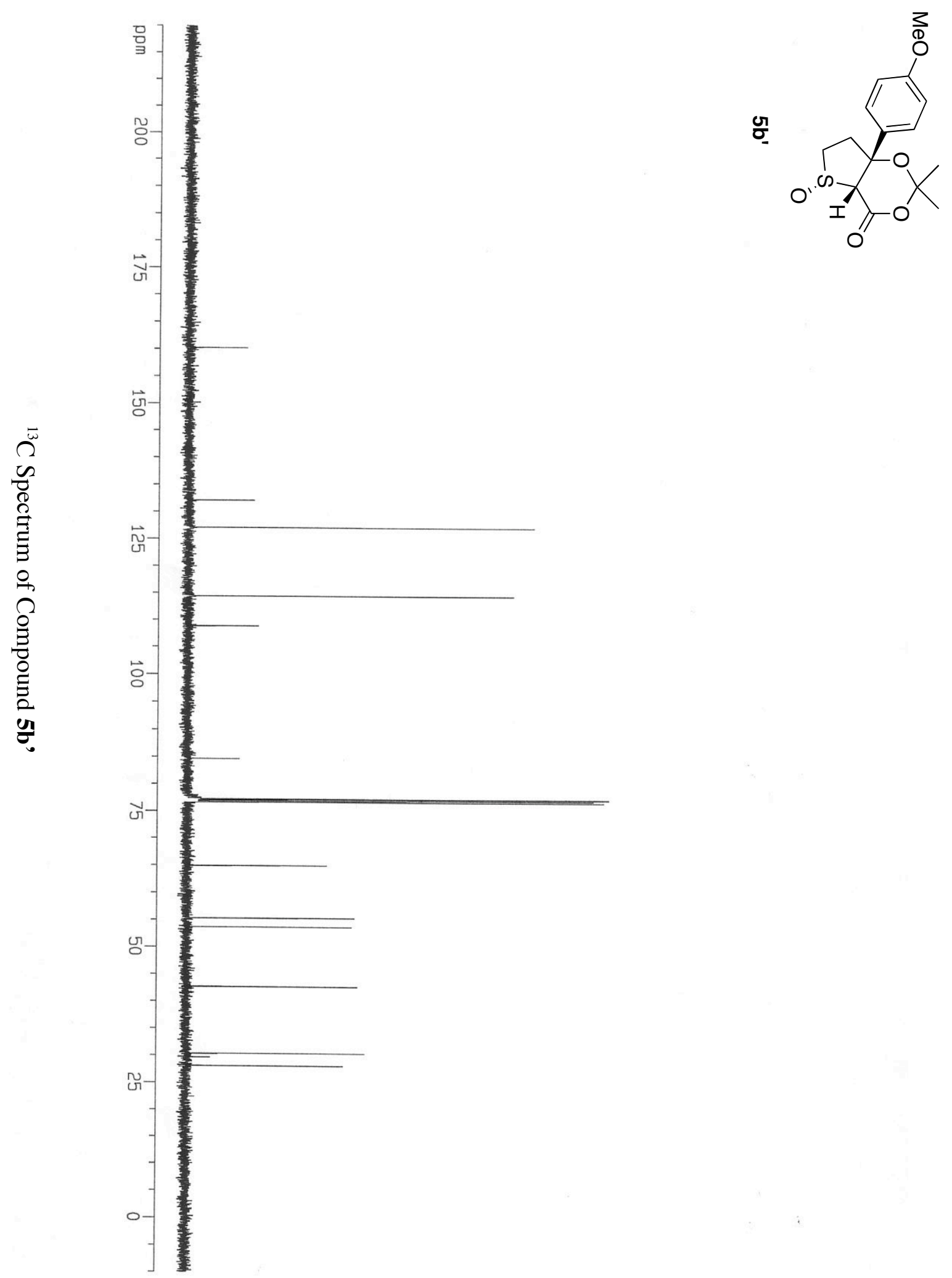


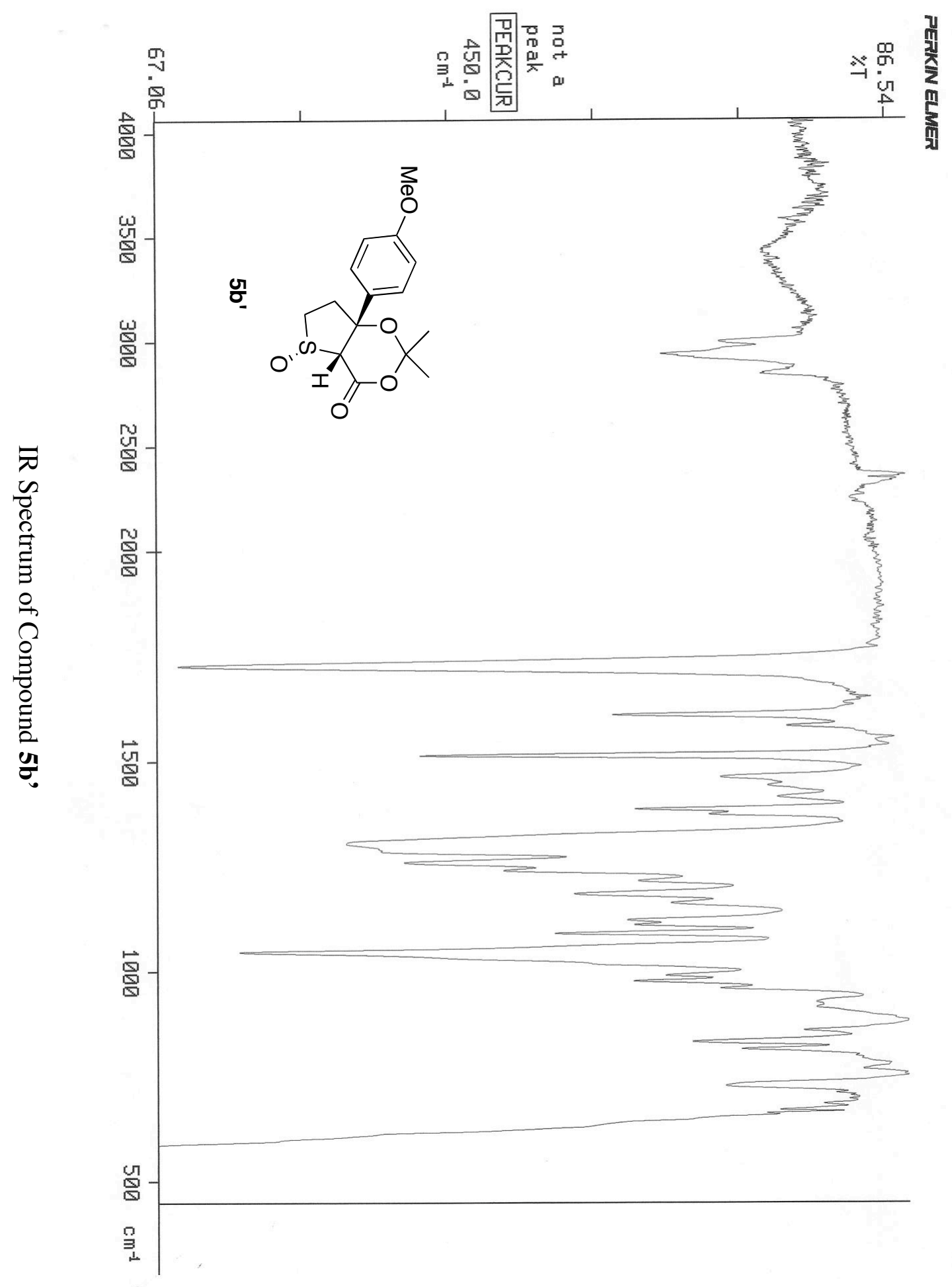



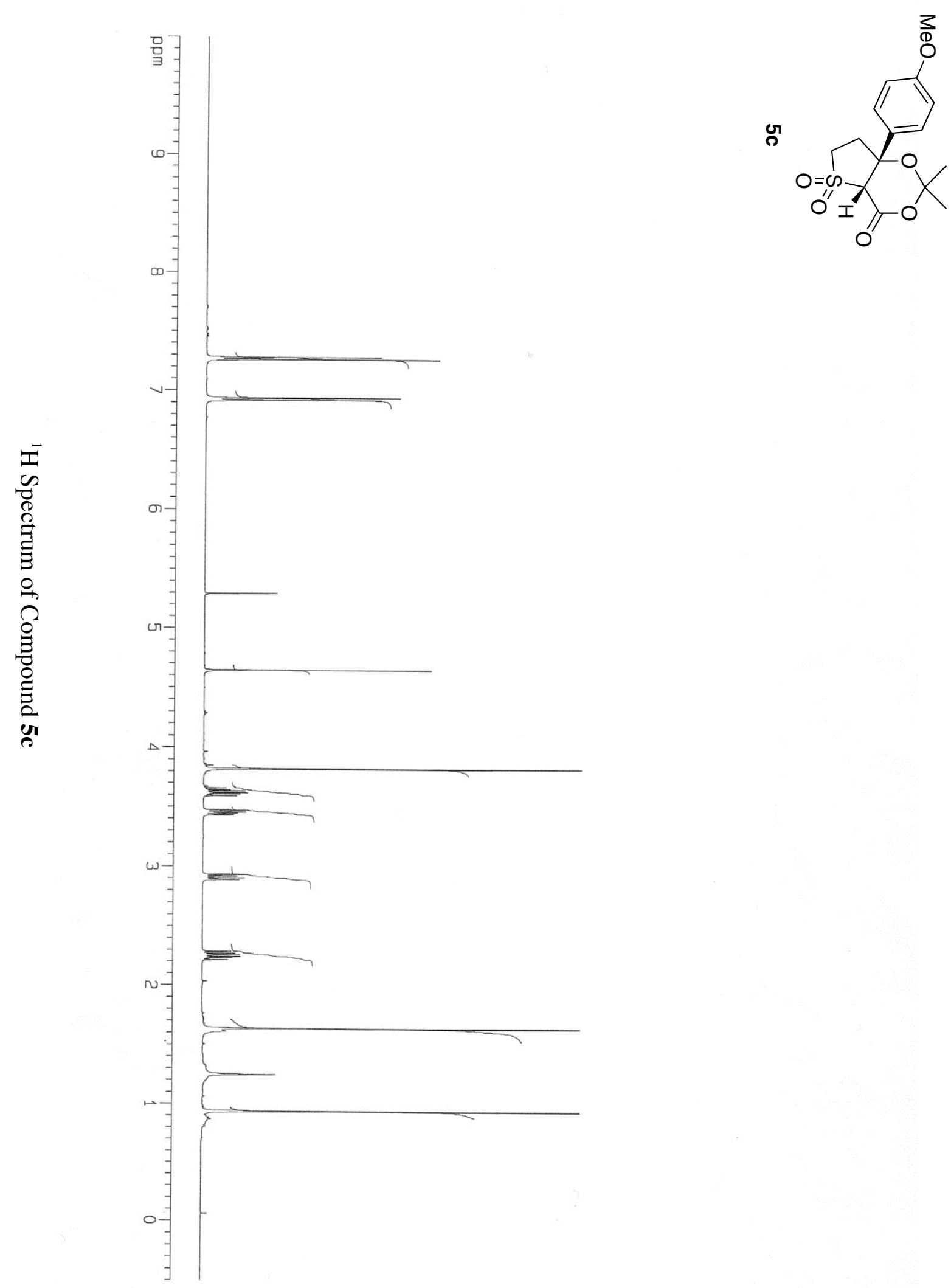

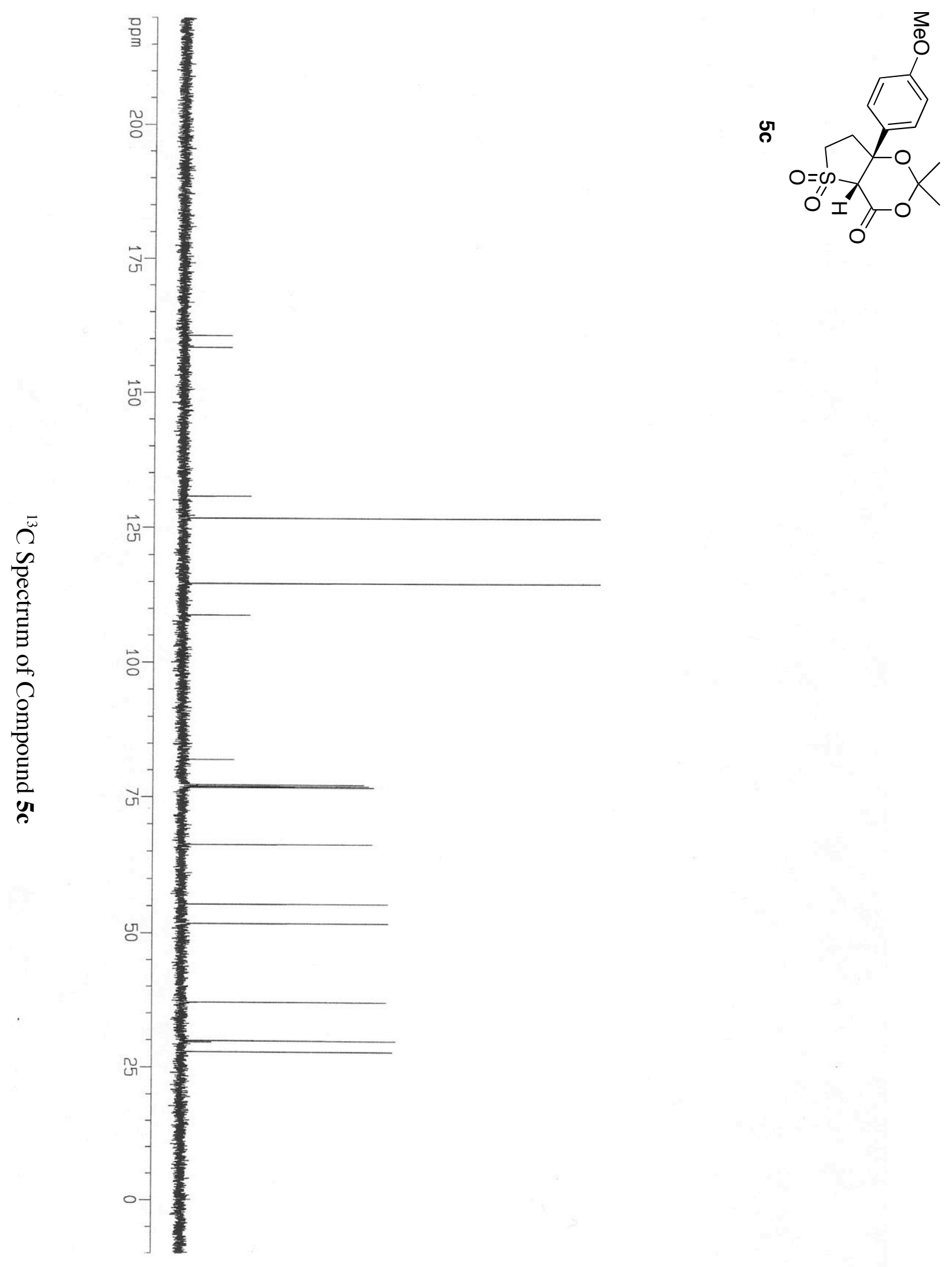


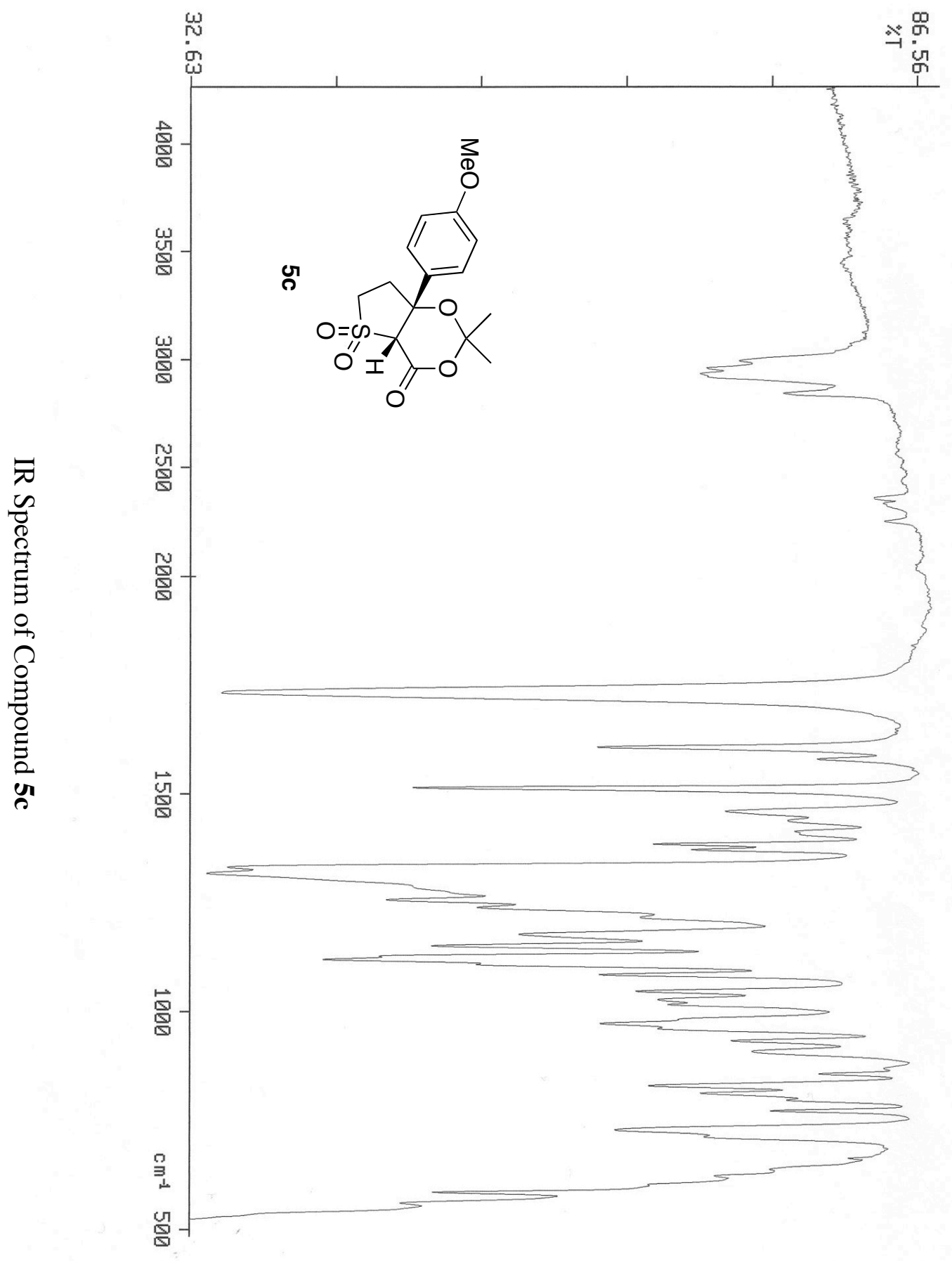



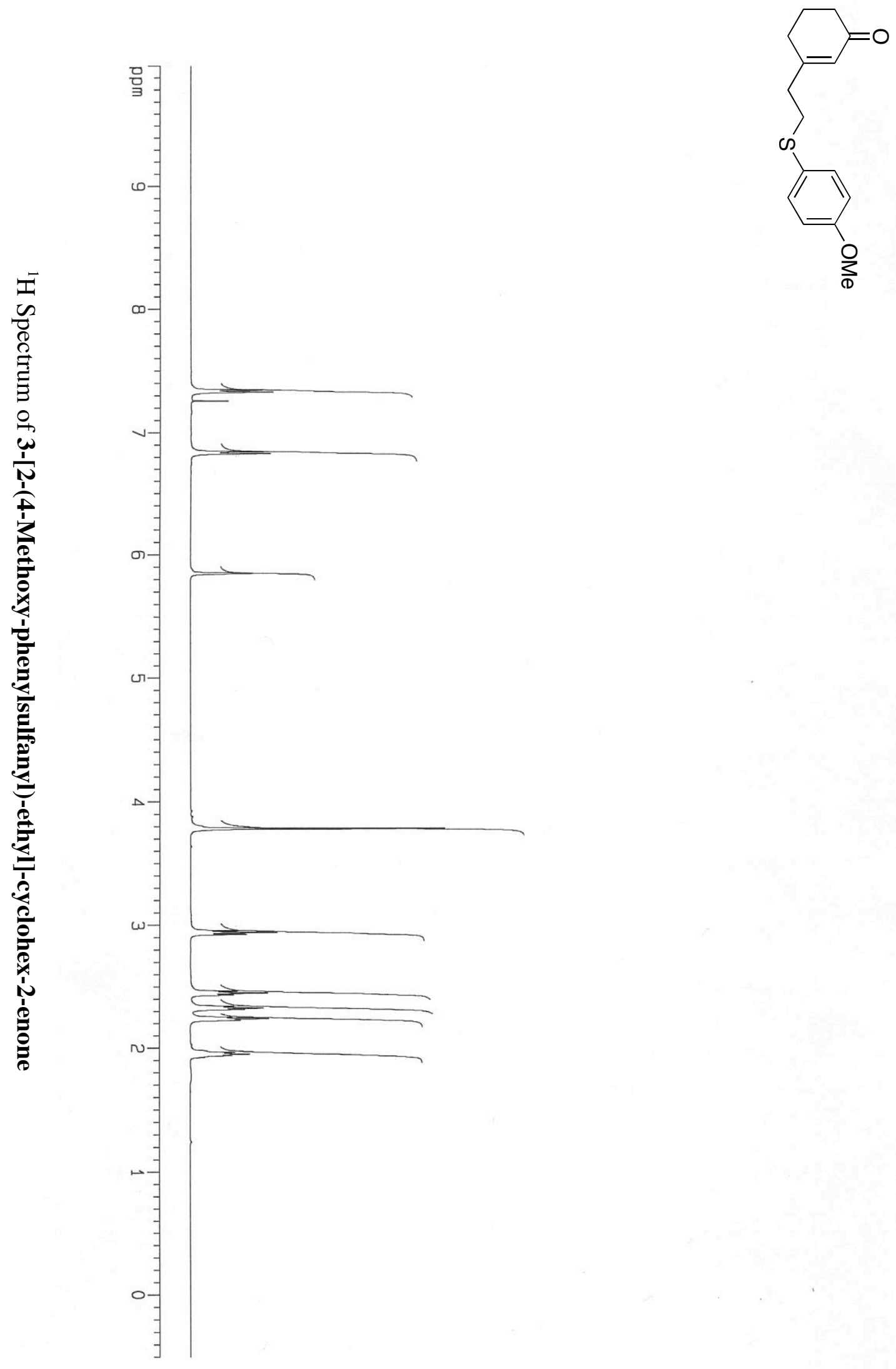

S-50 

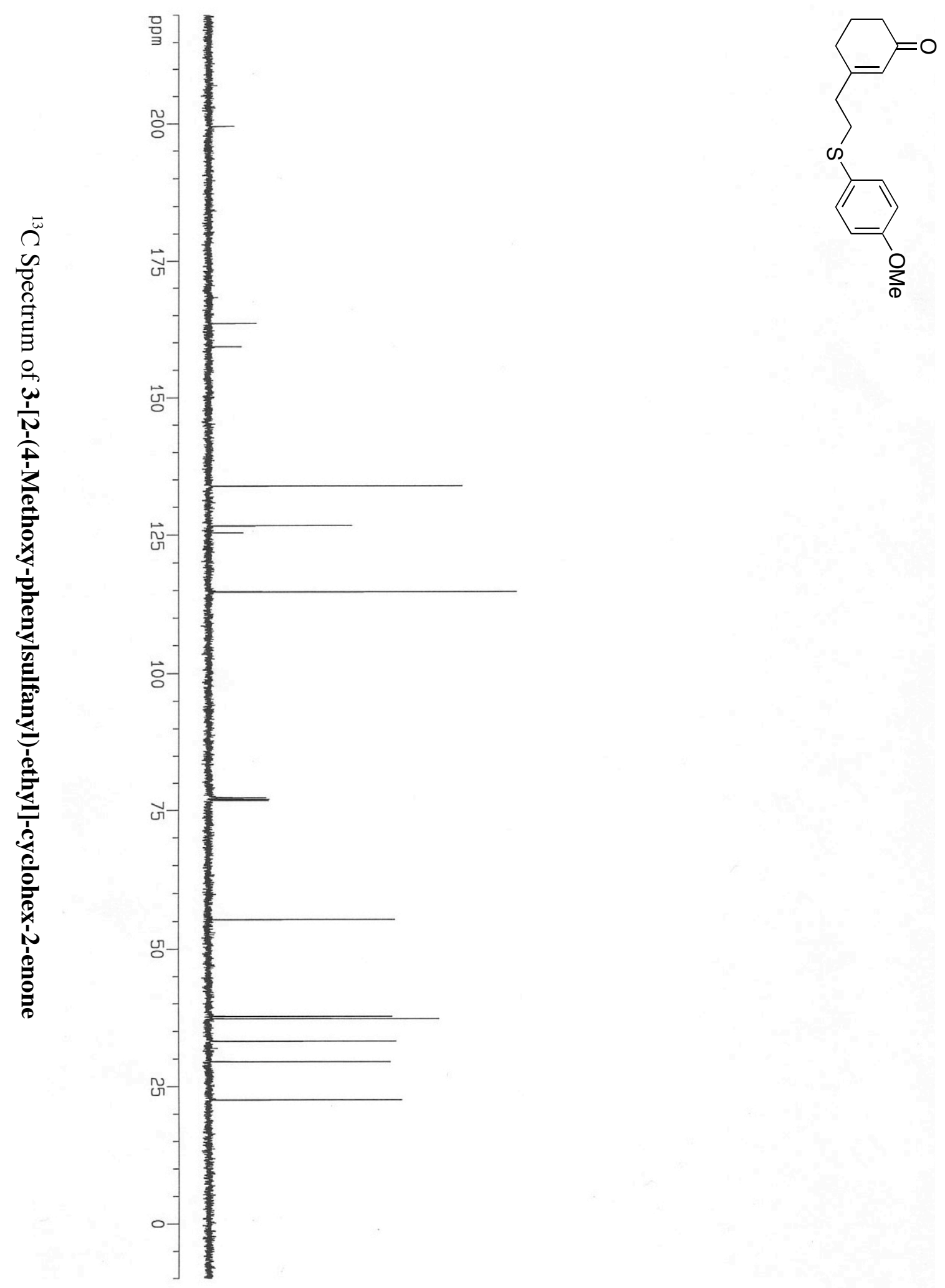

S-51 


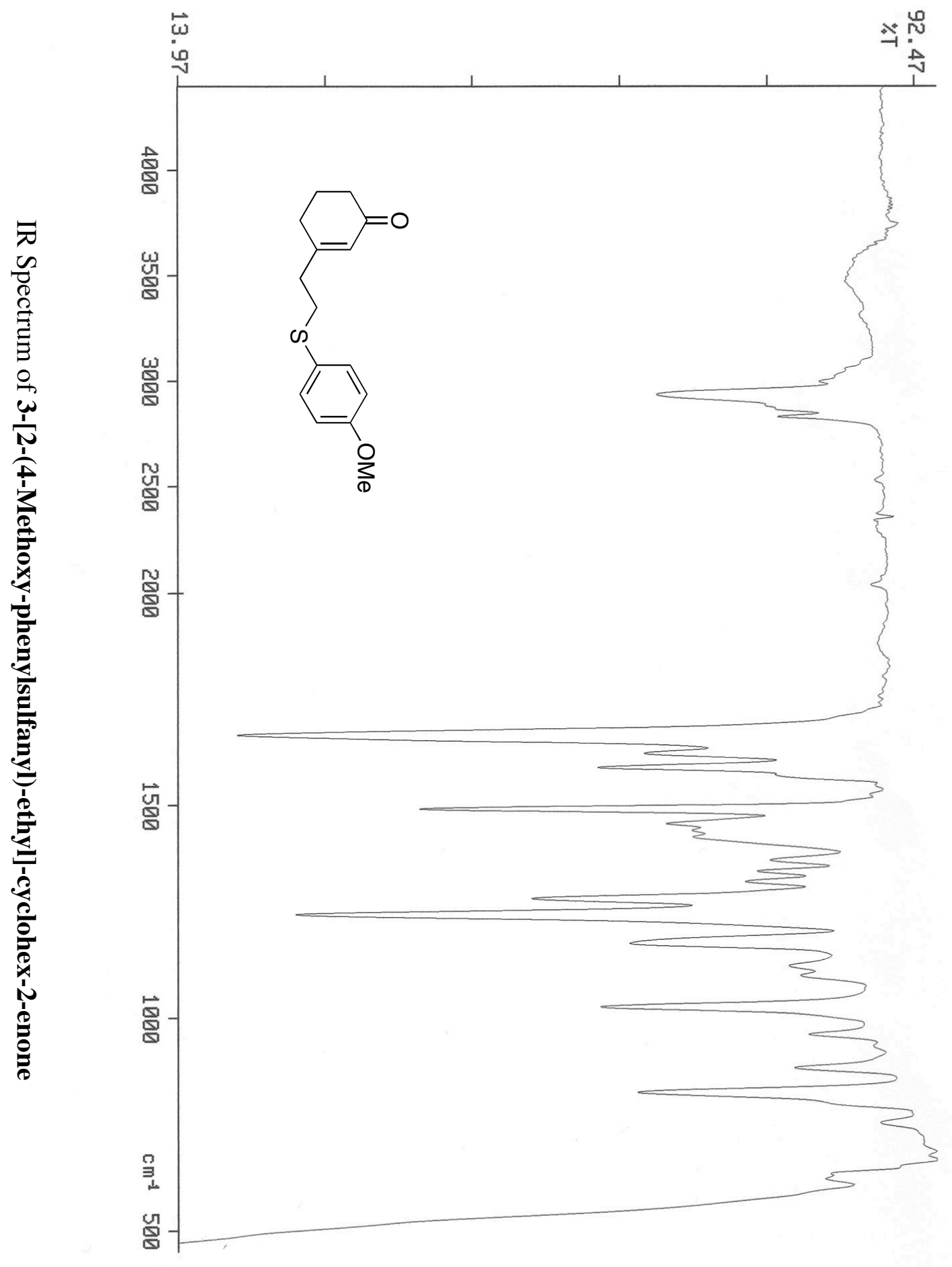

S- 52 

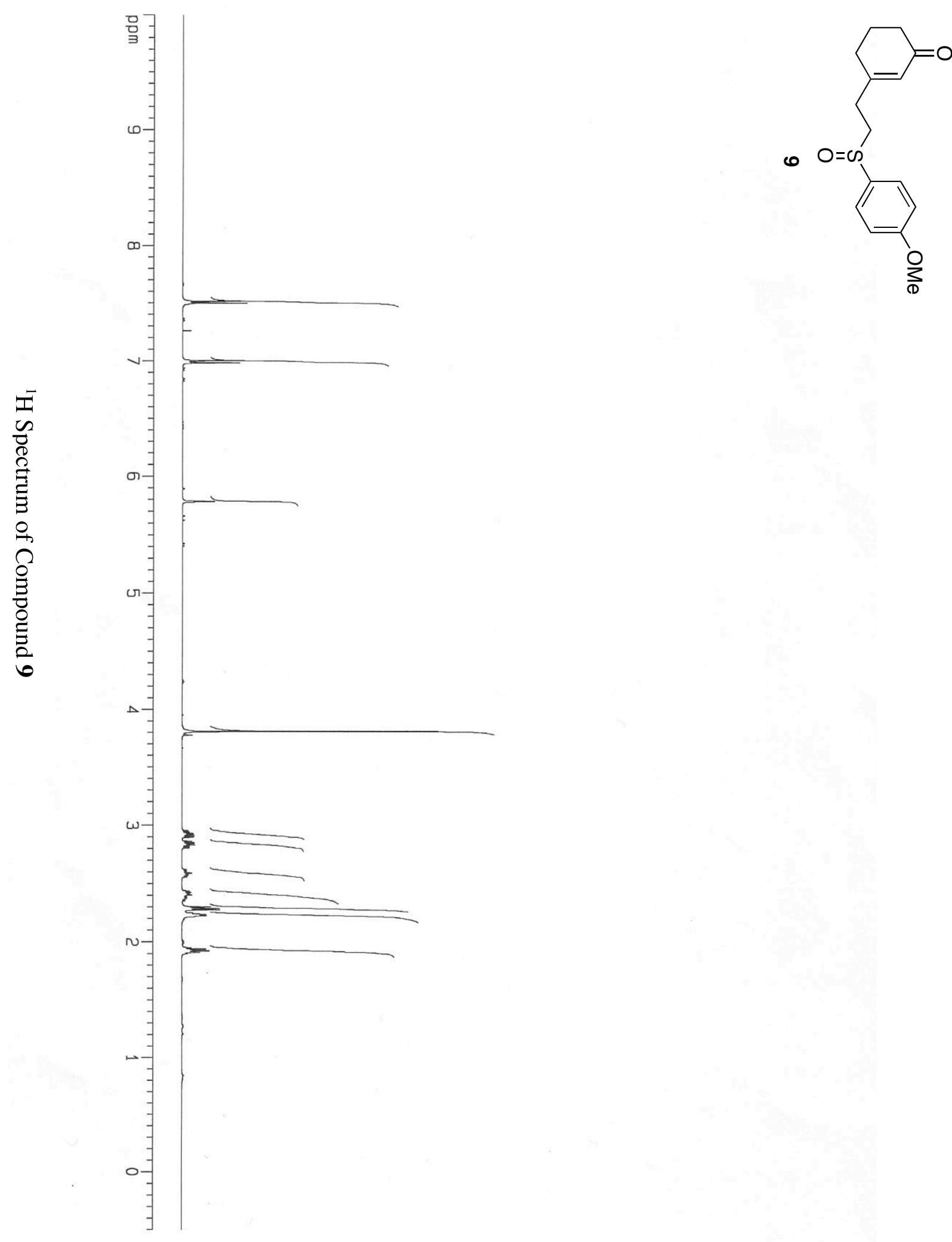

S-.53 


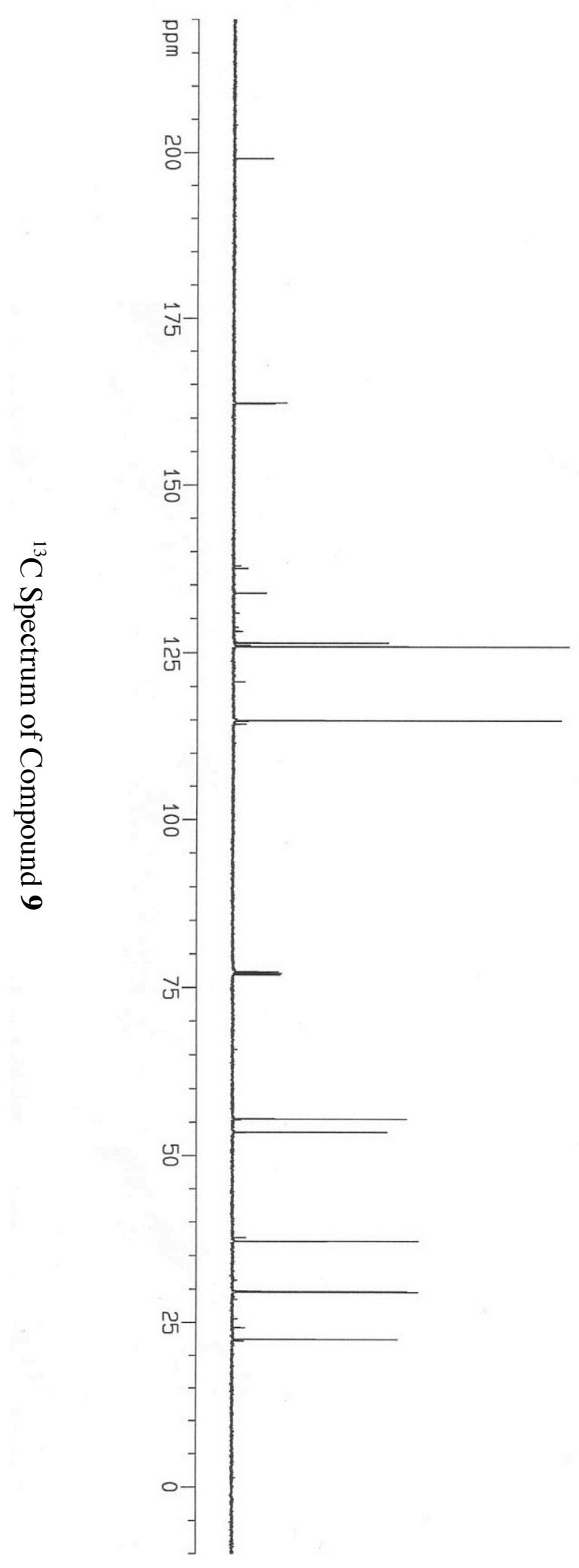




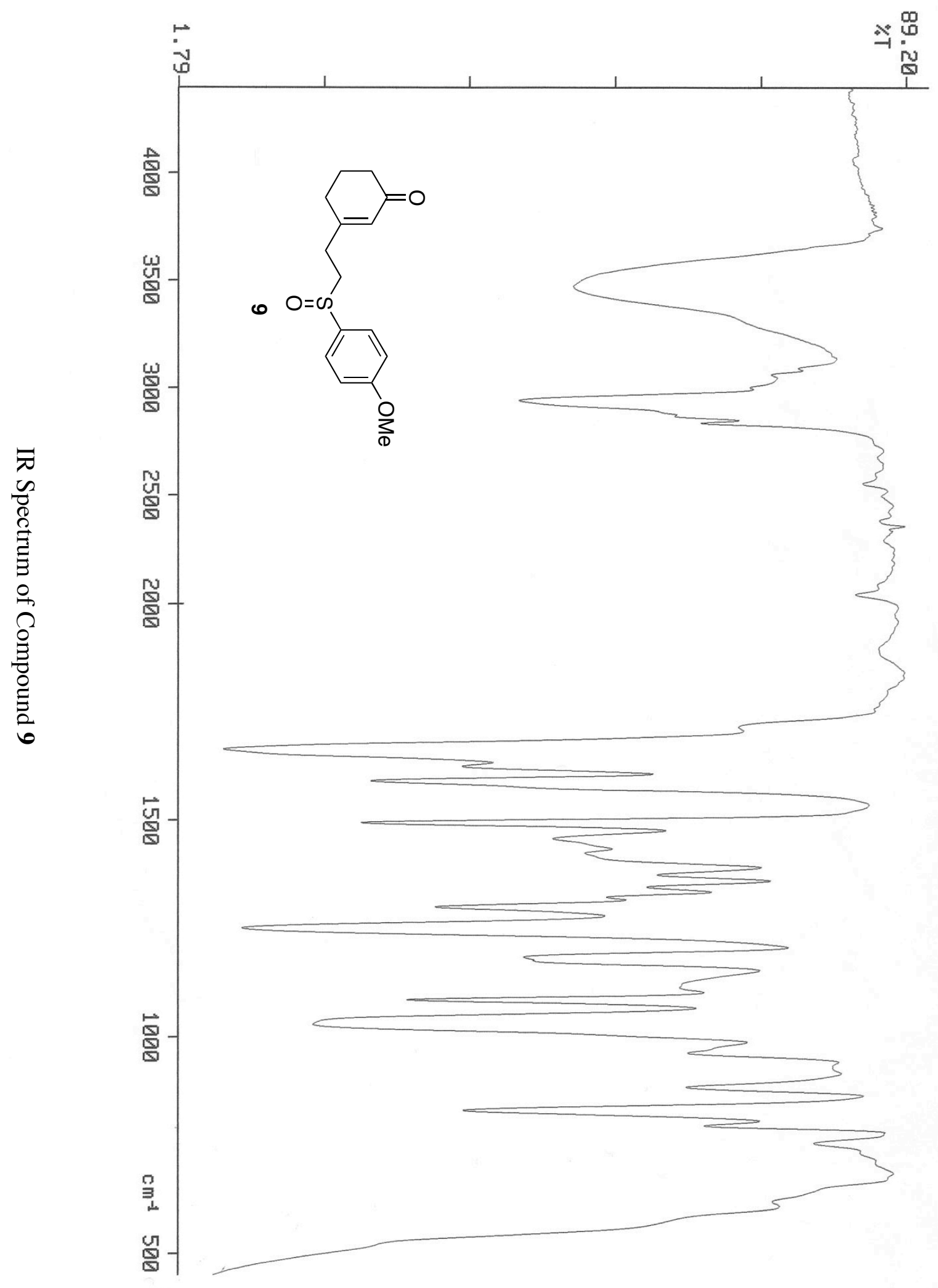

S-55 

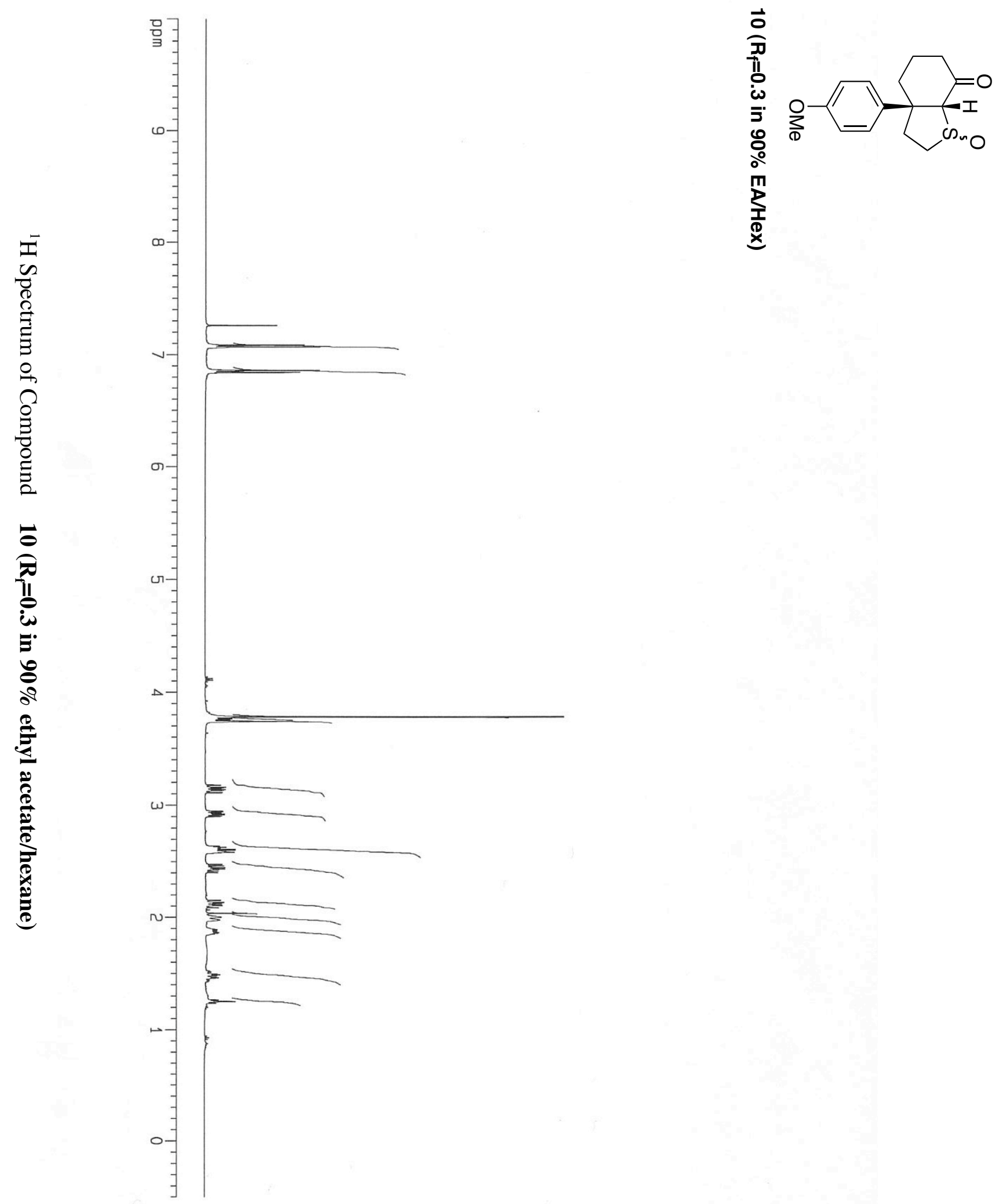

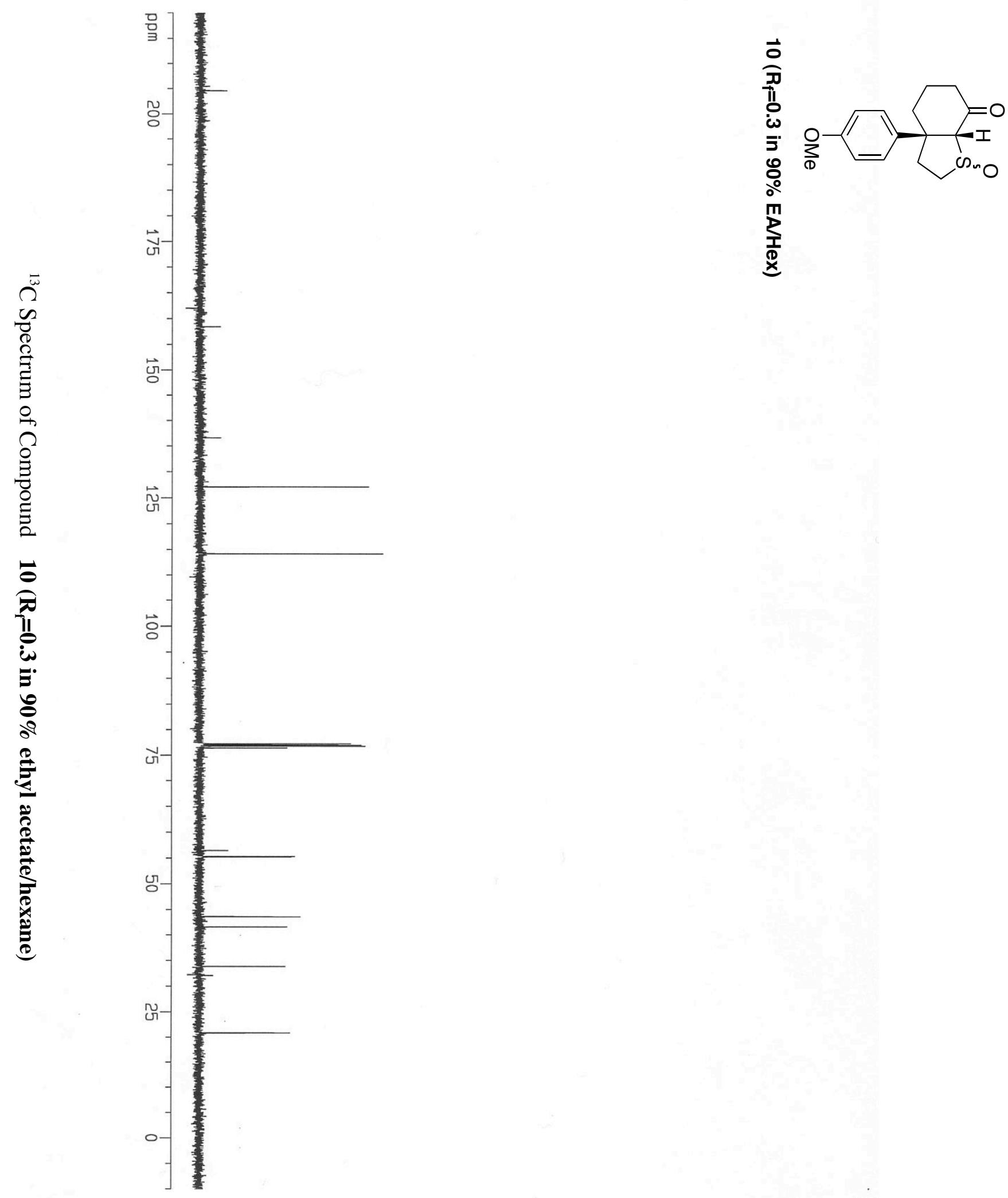


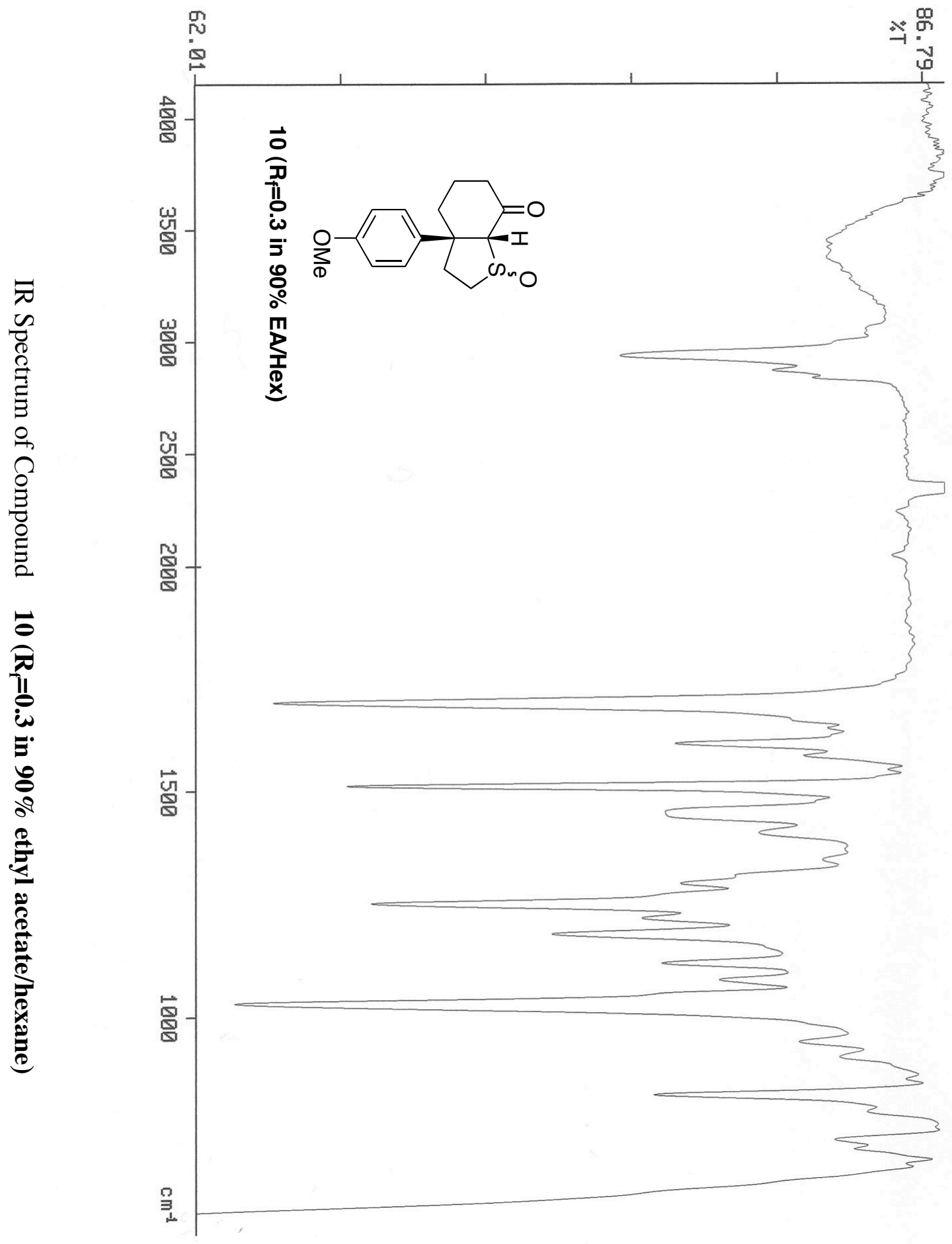



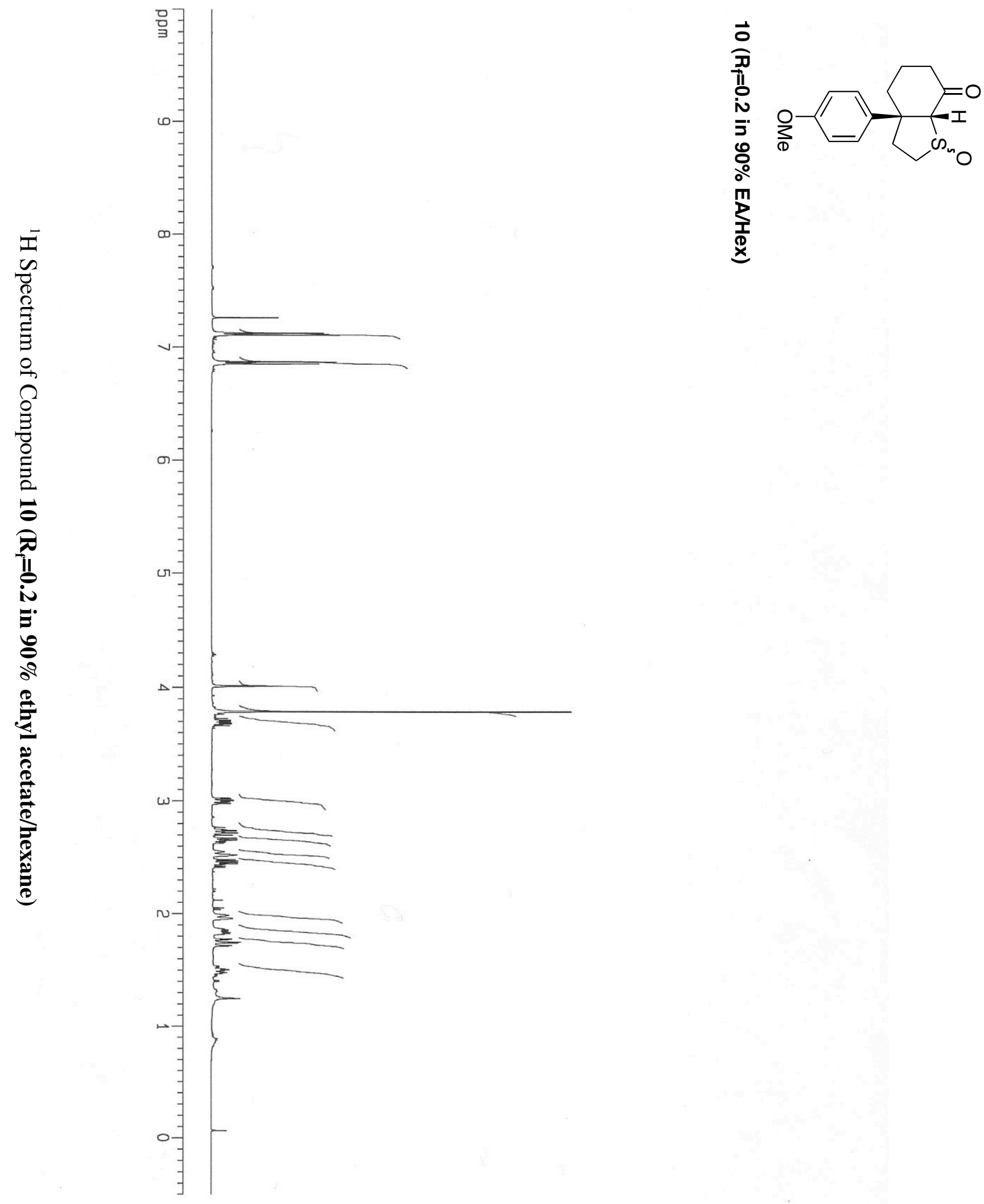

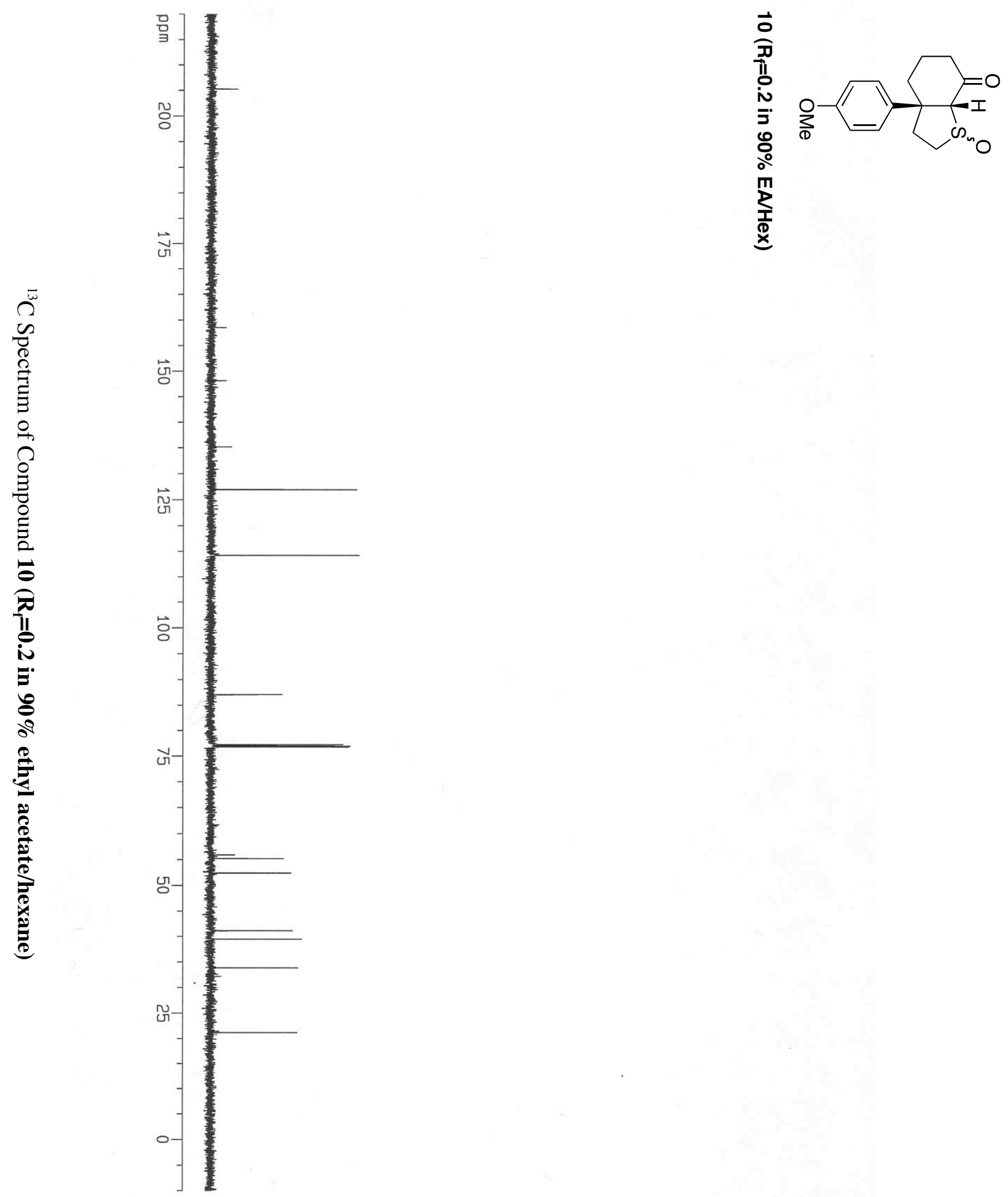


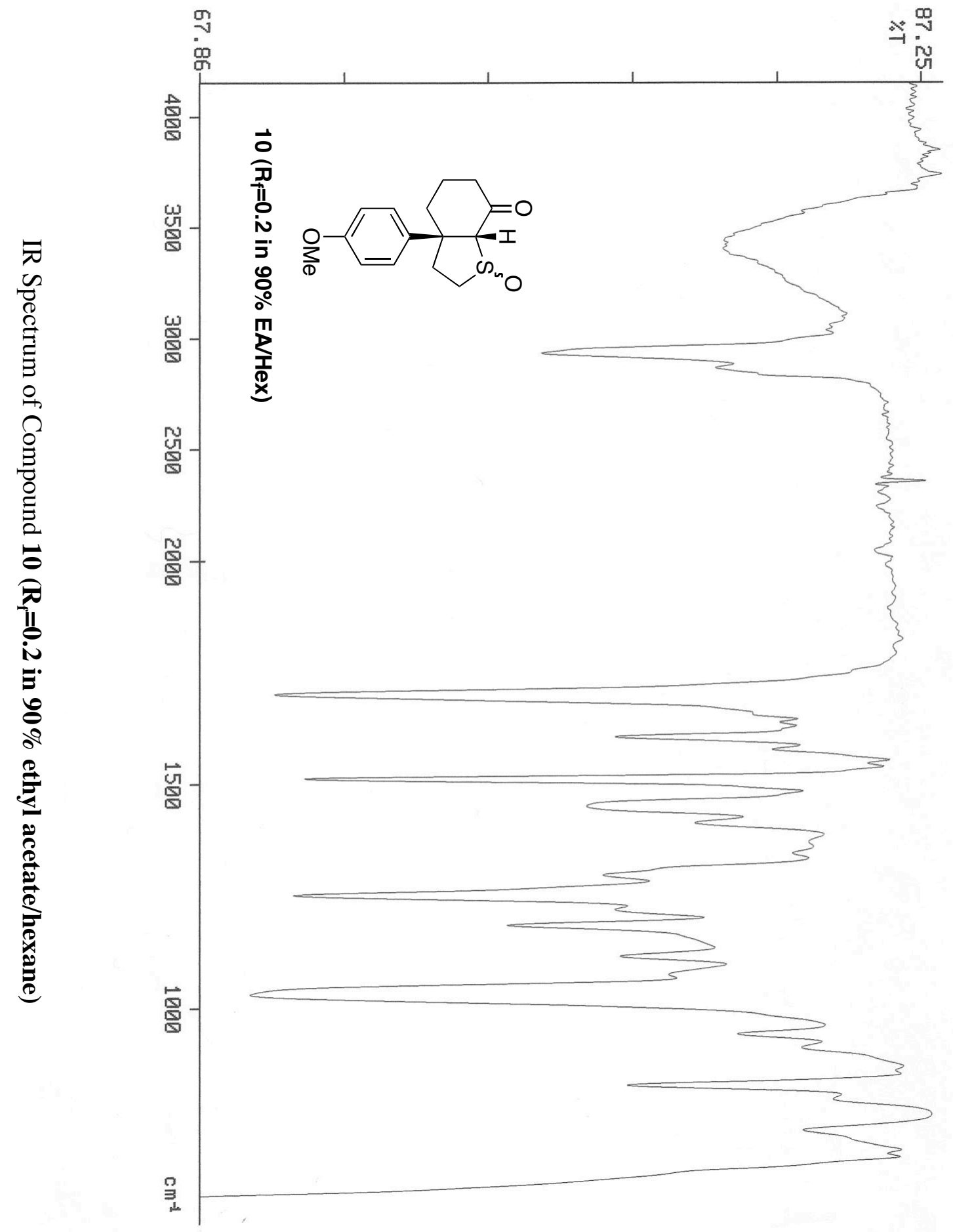




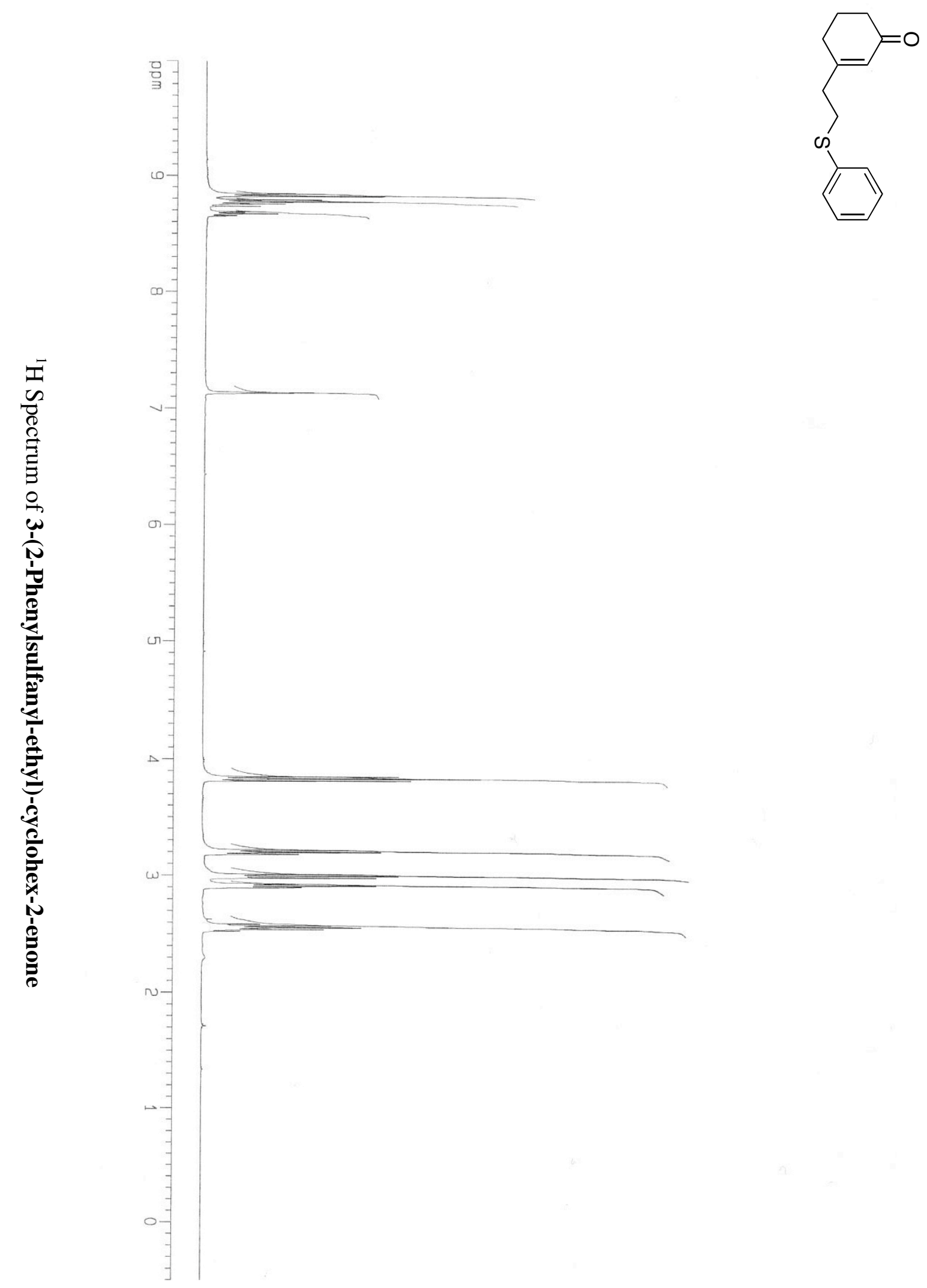

S-62 

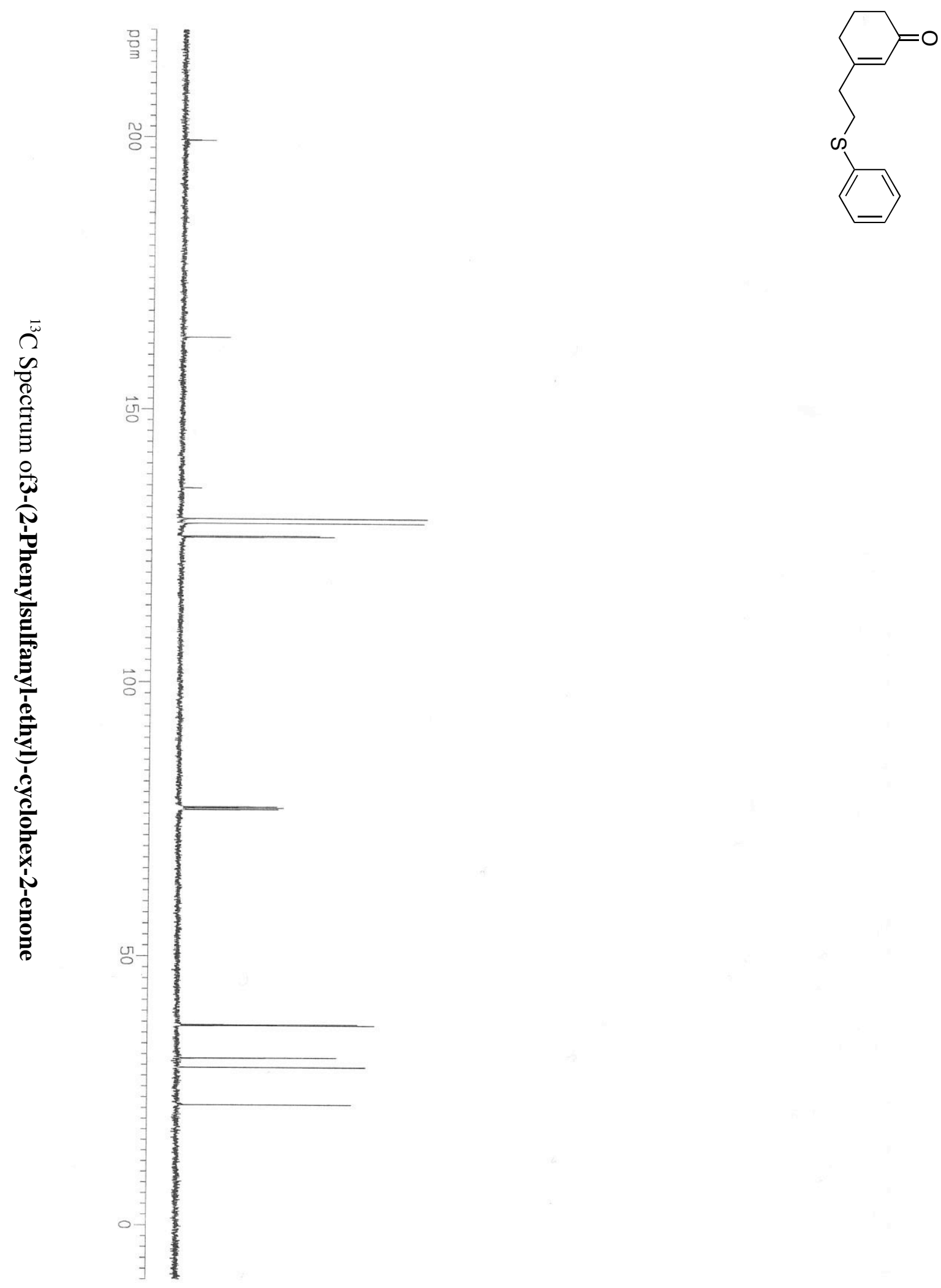

S-63 


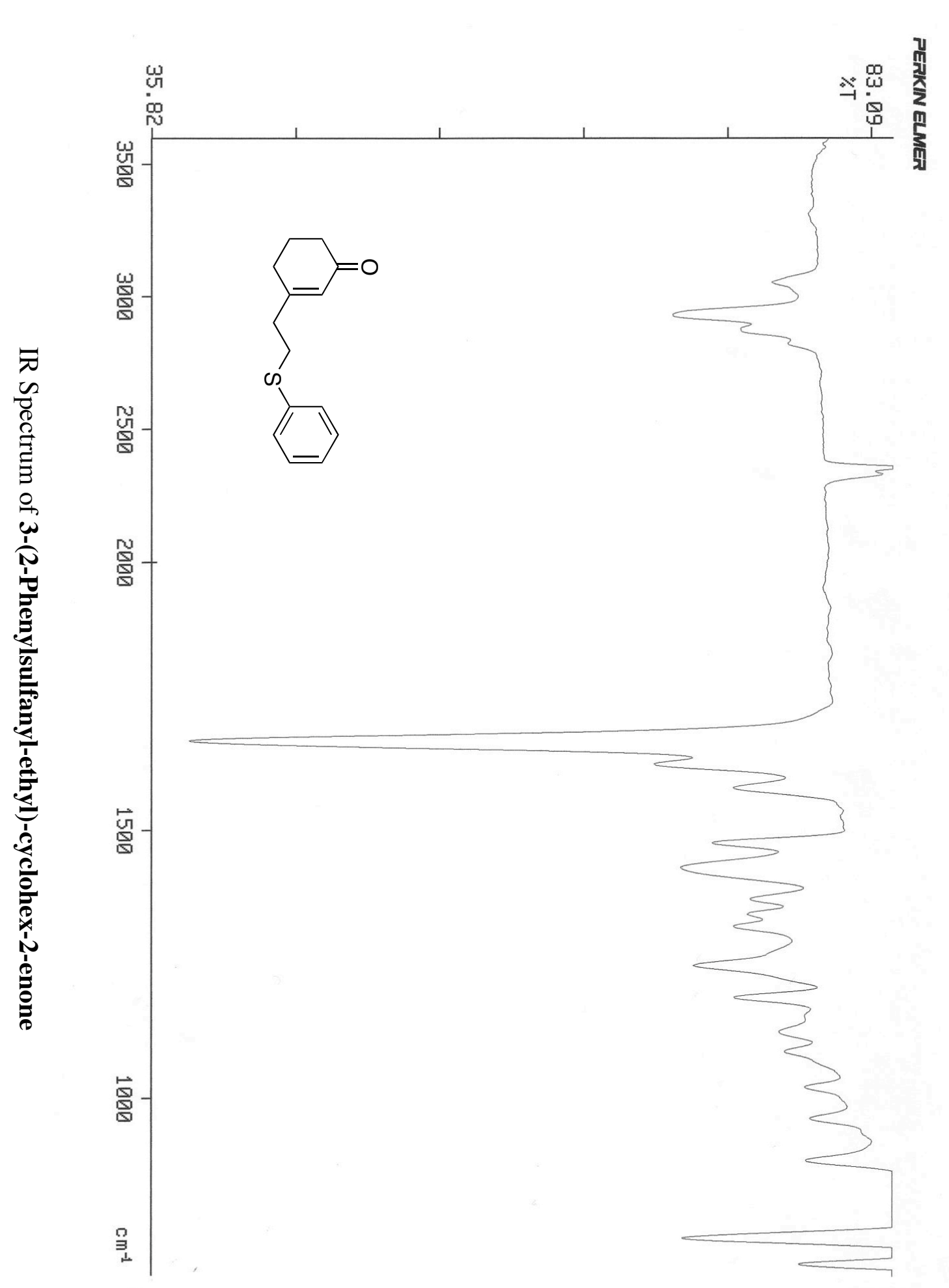



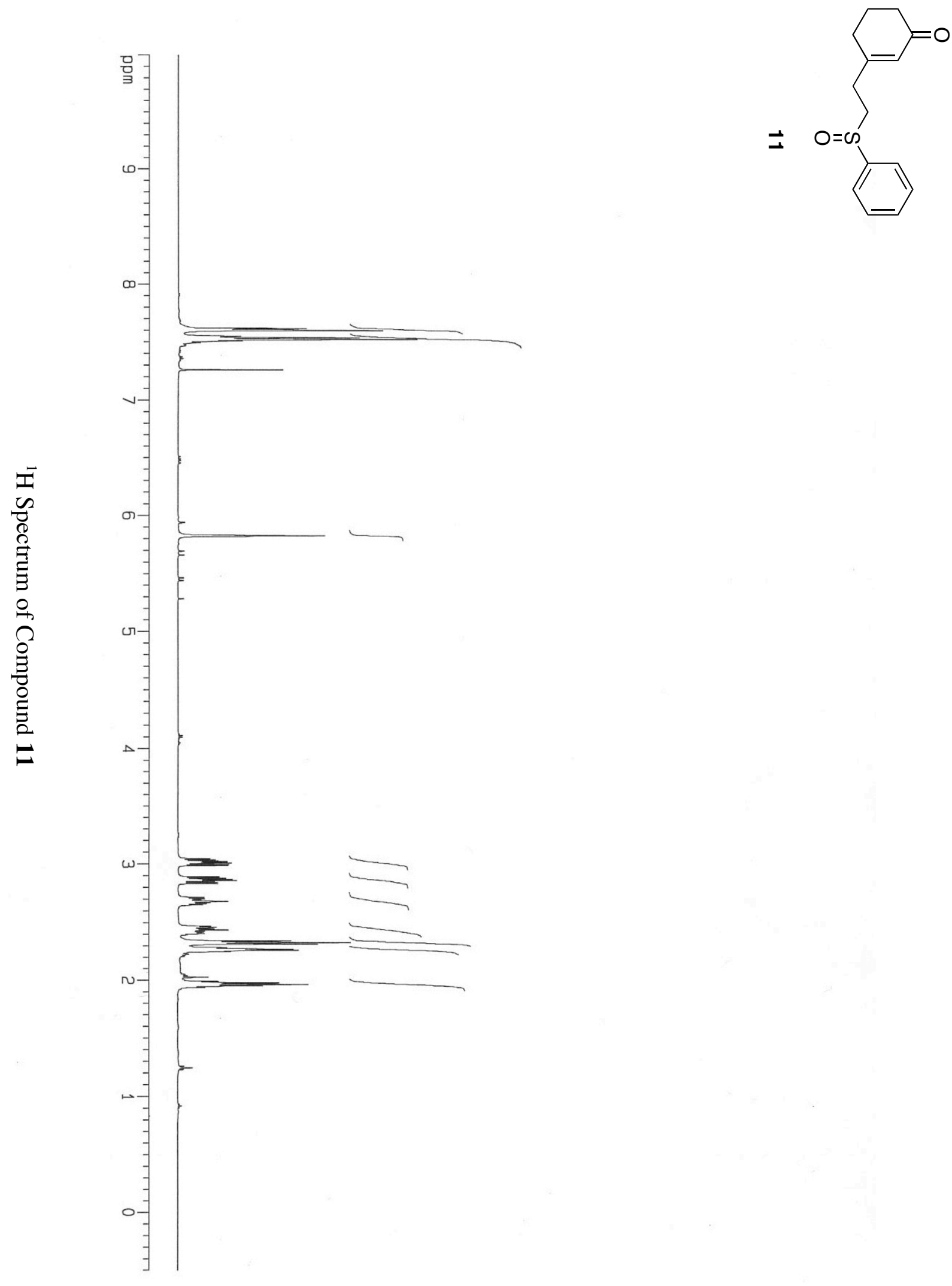

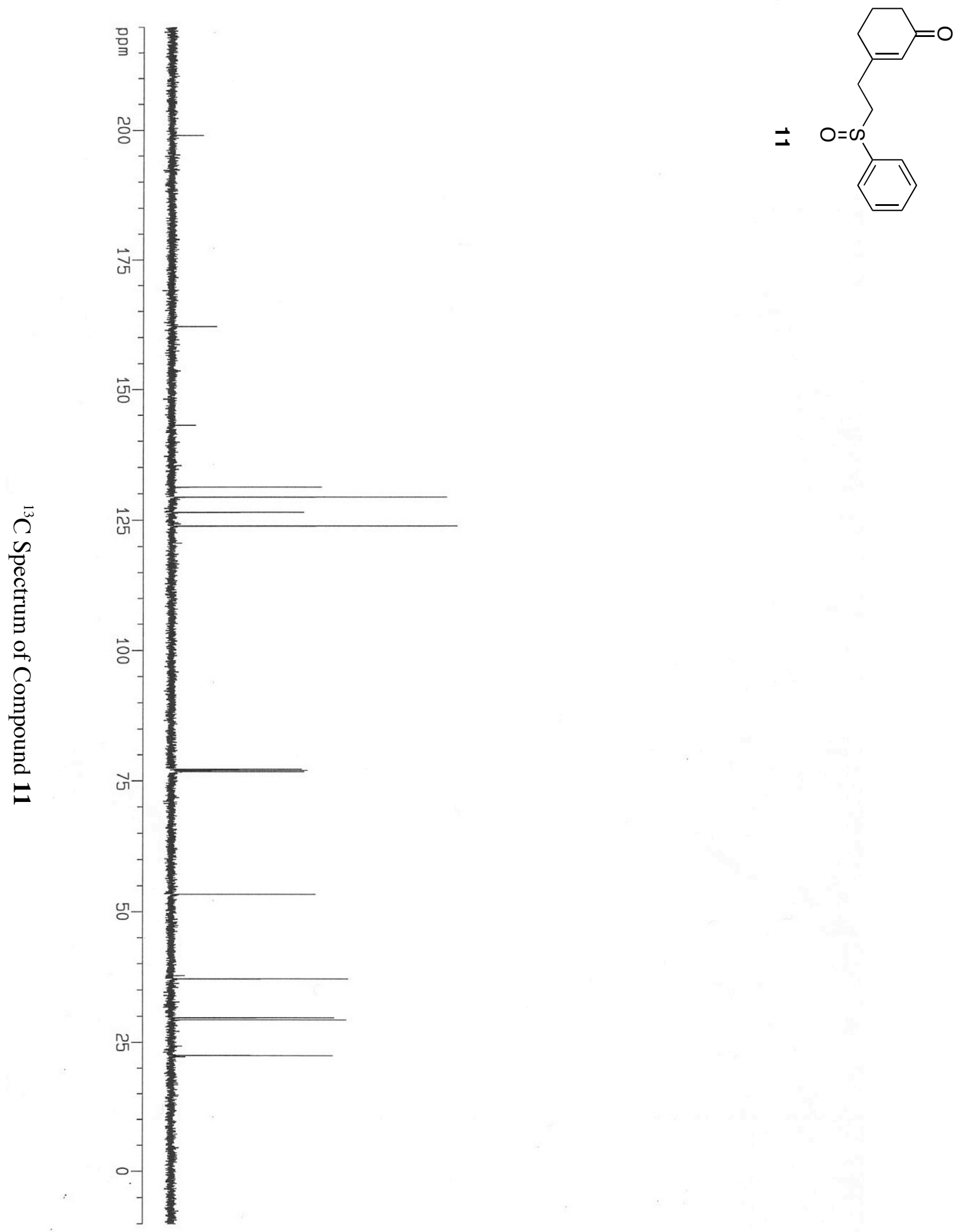

S-66 


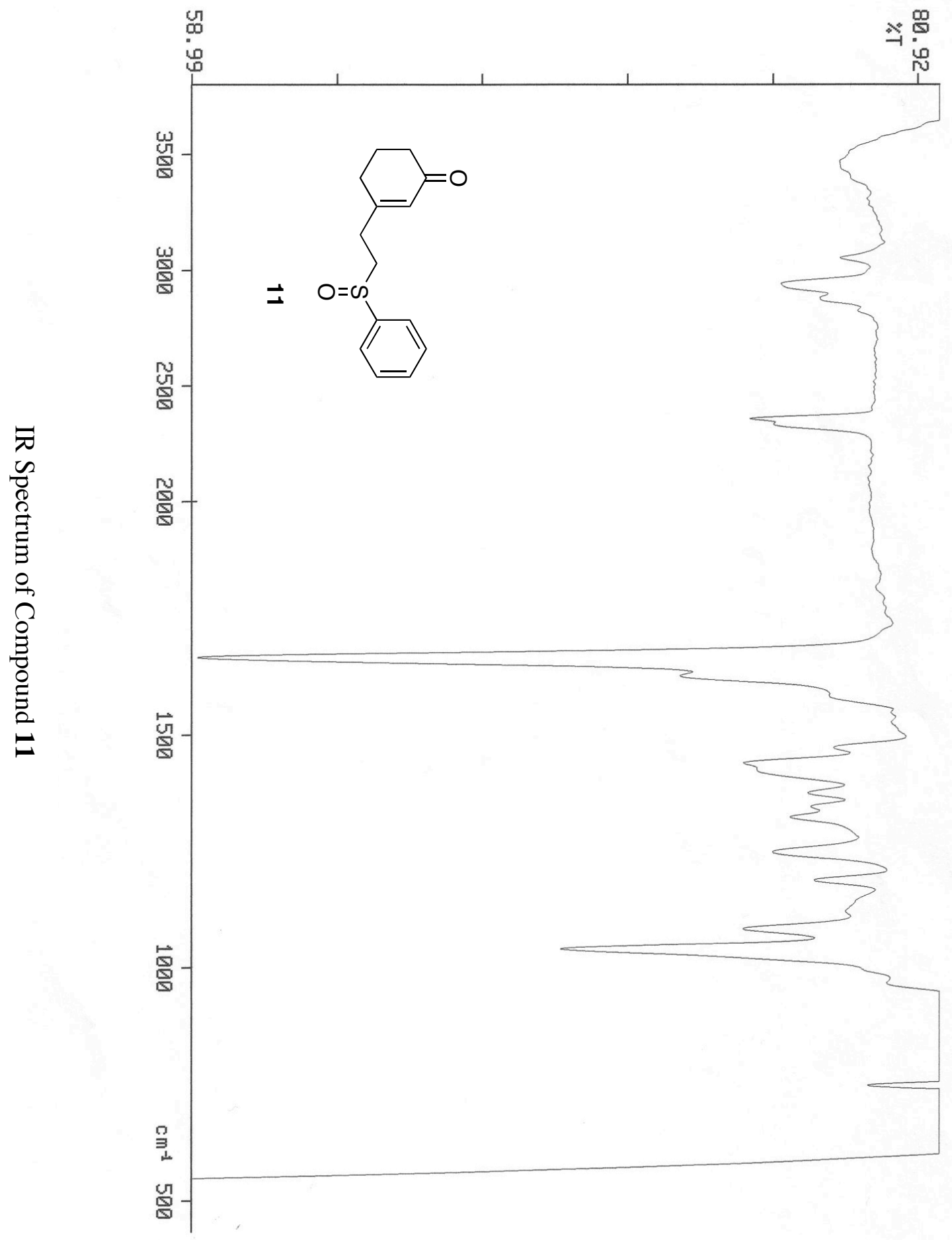



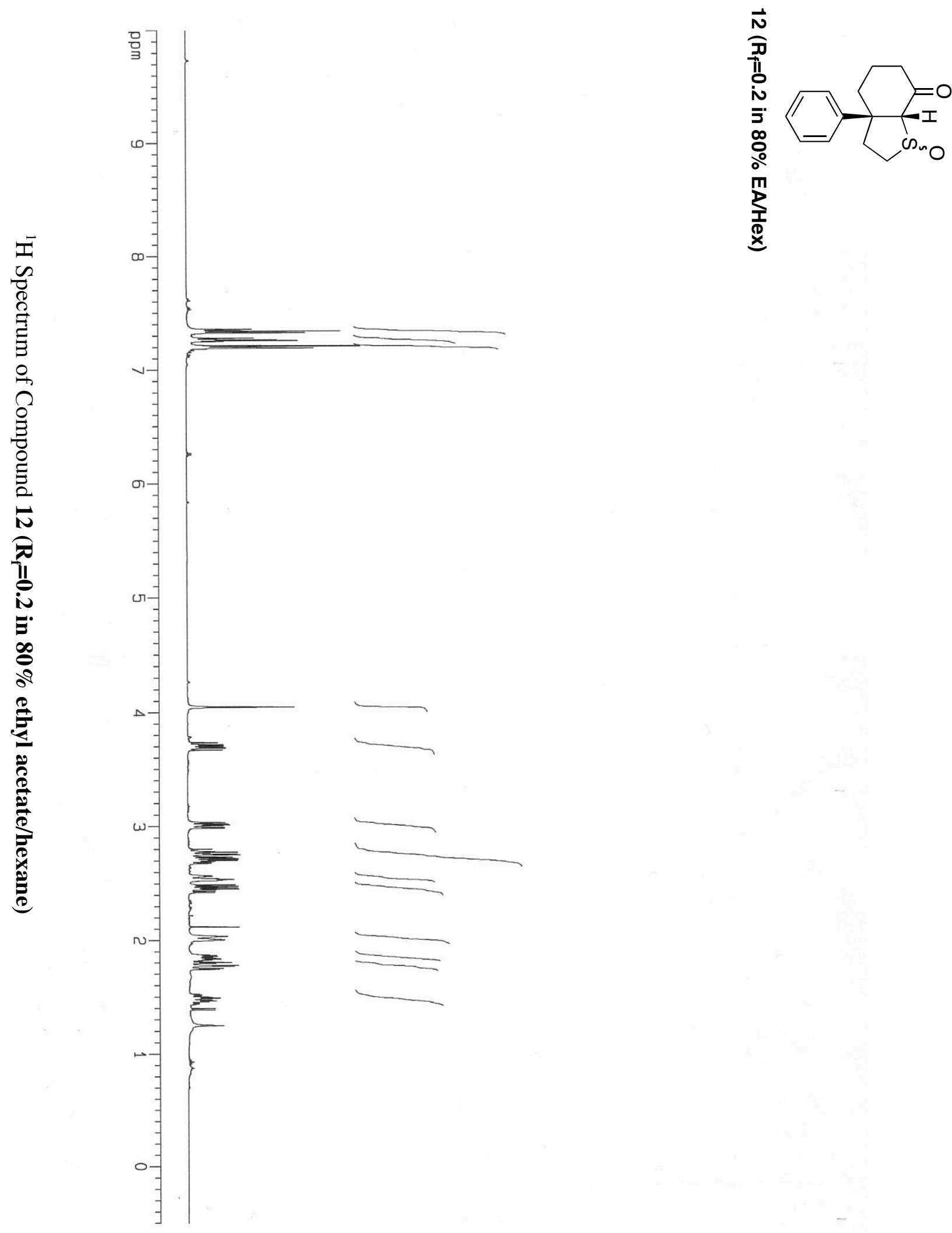

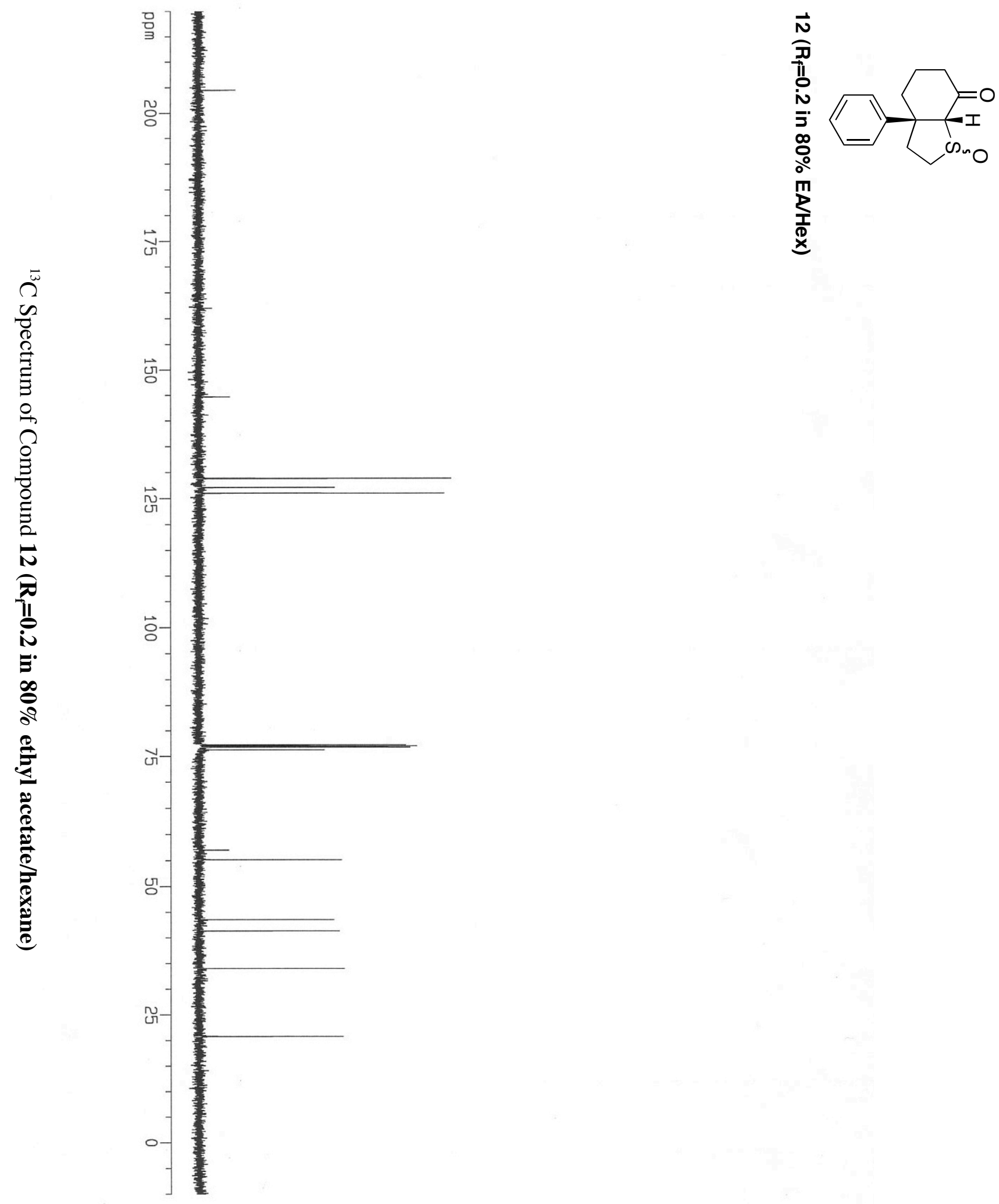


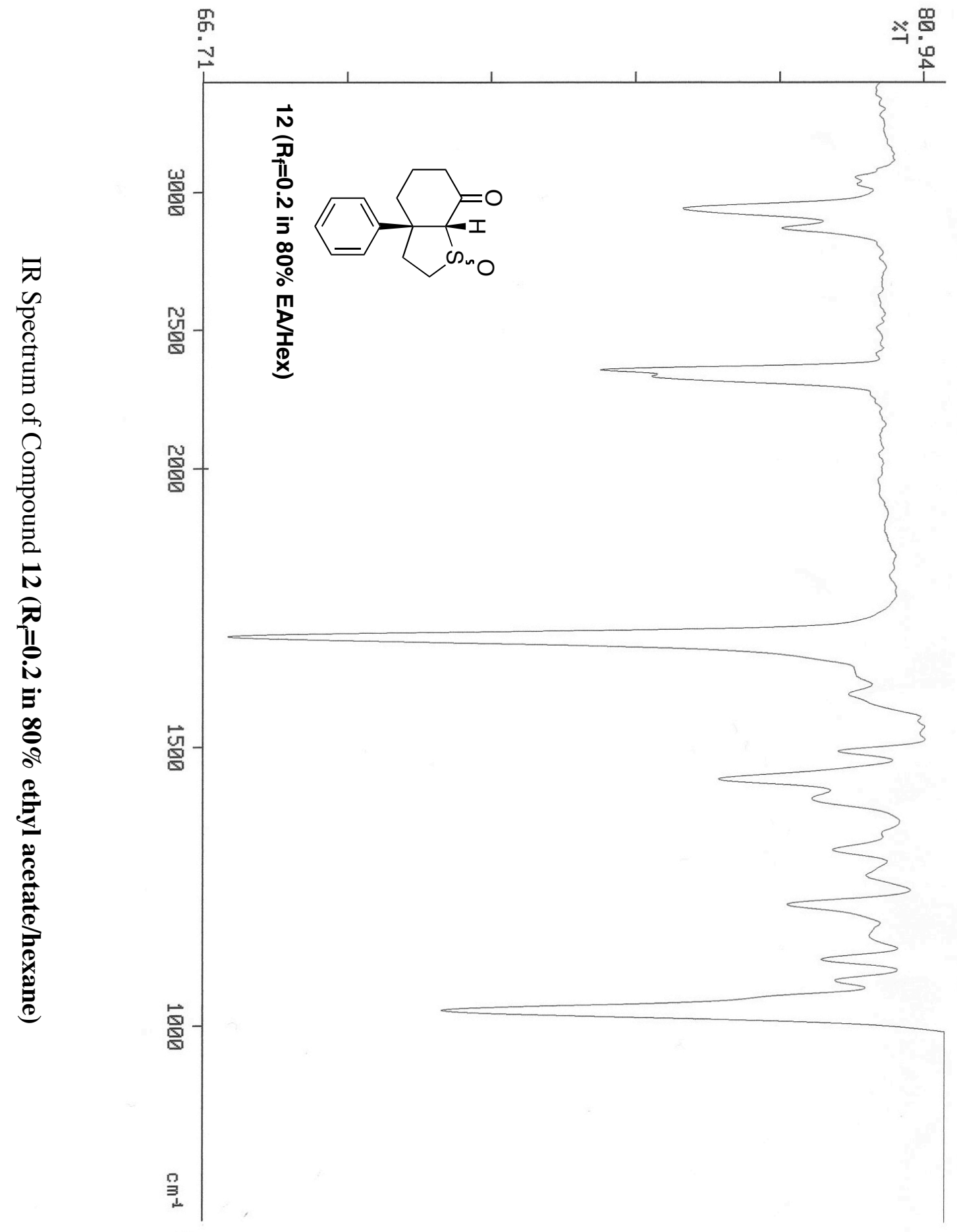



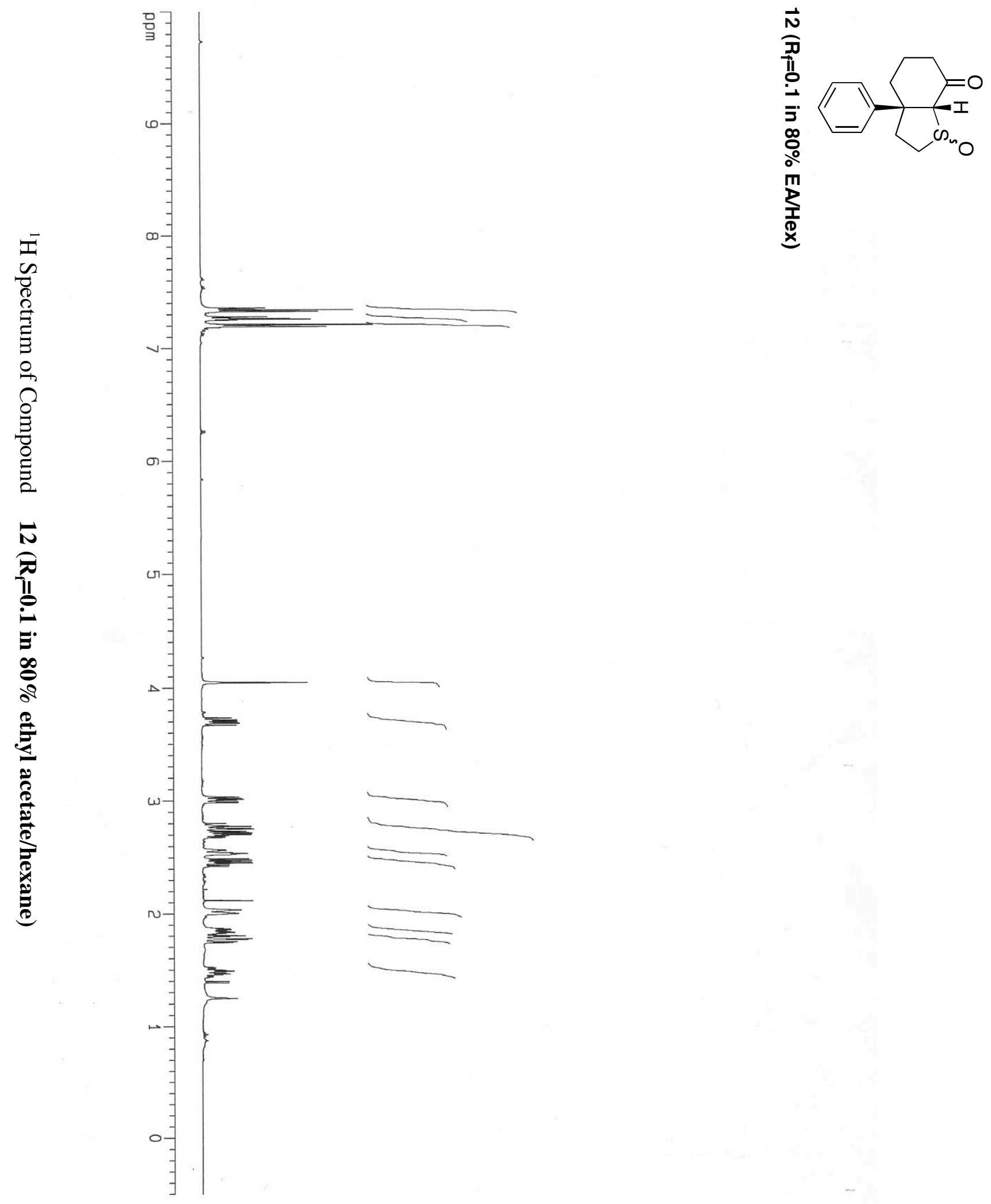

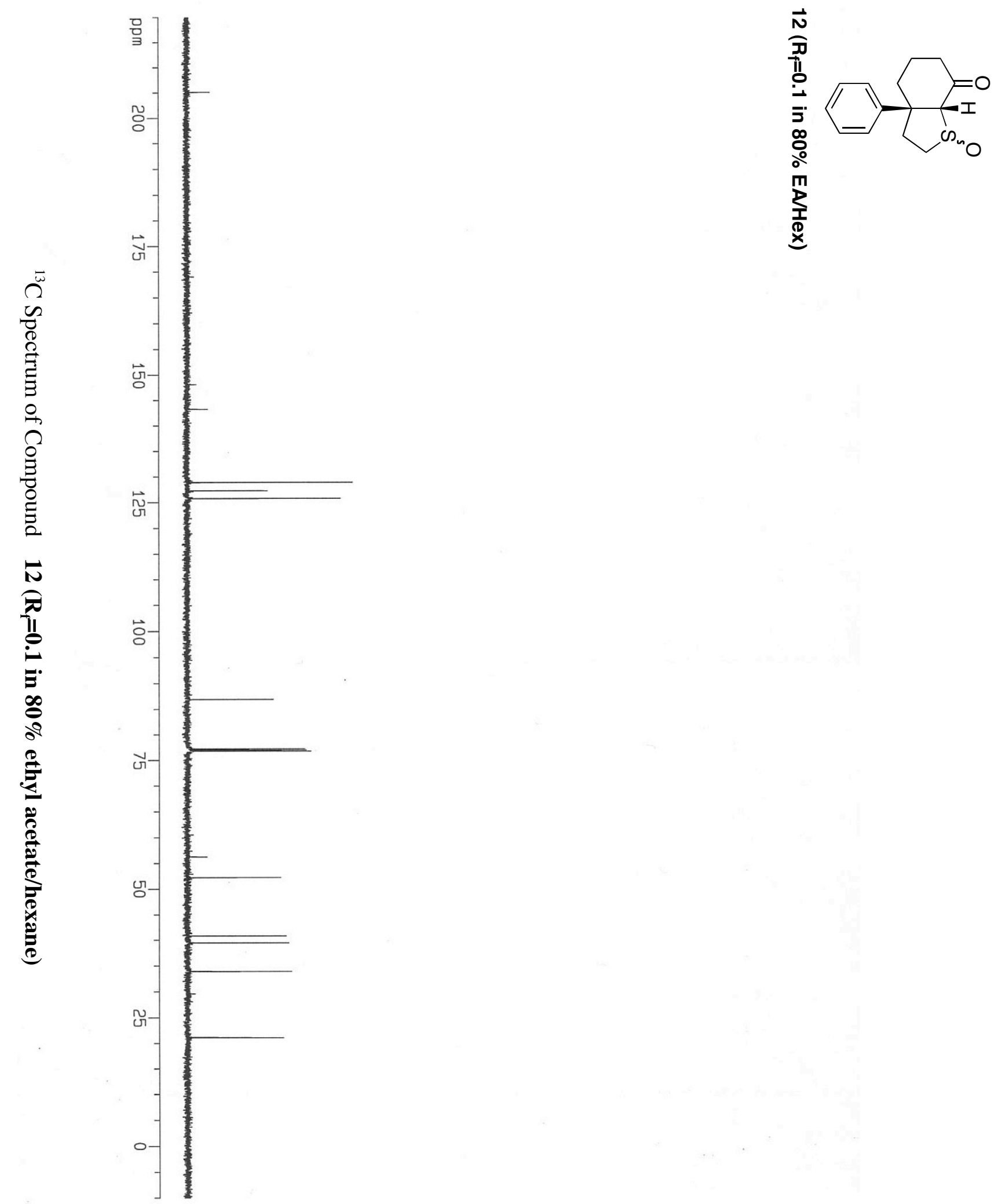


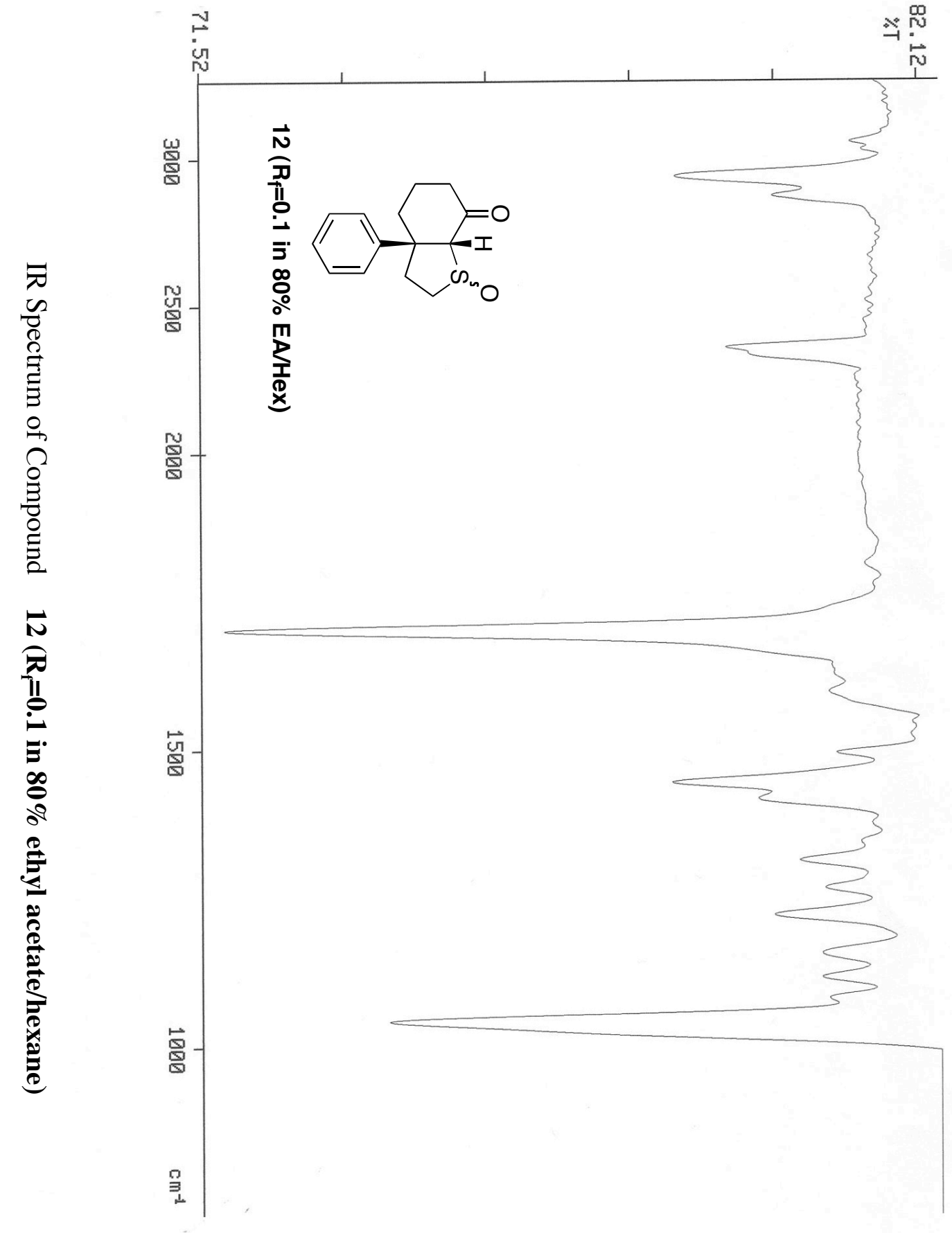

S-73 


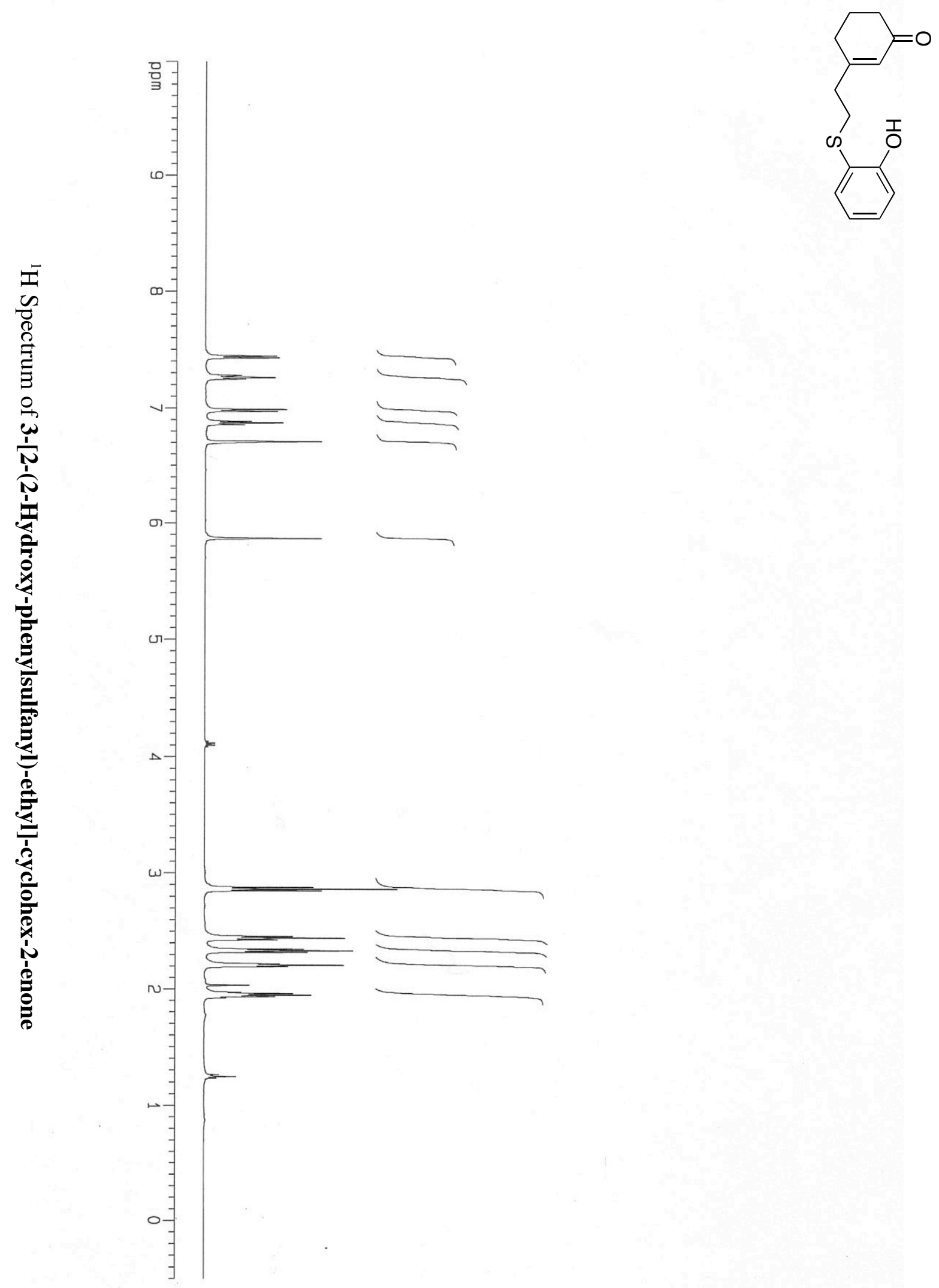



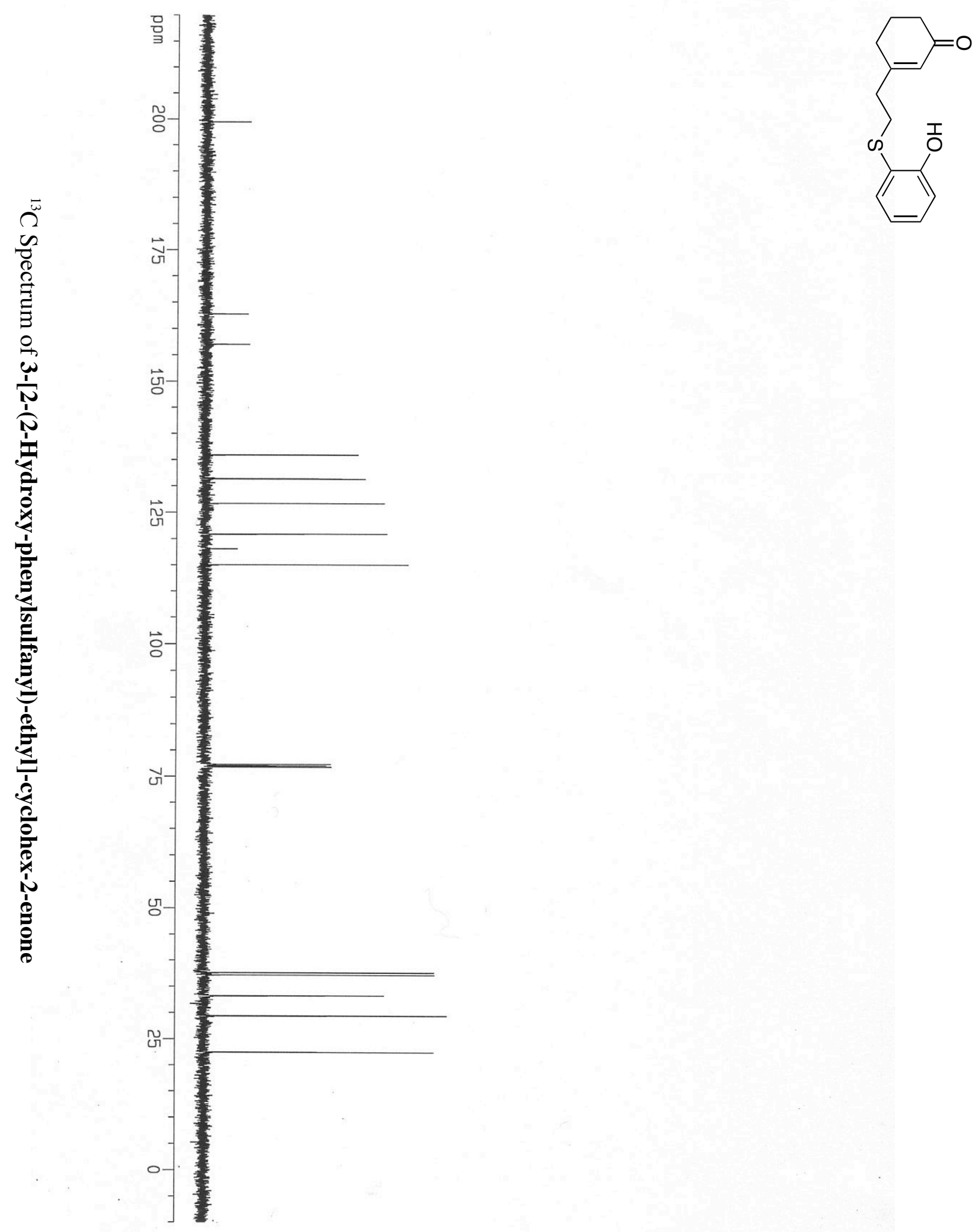


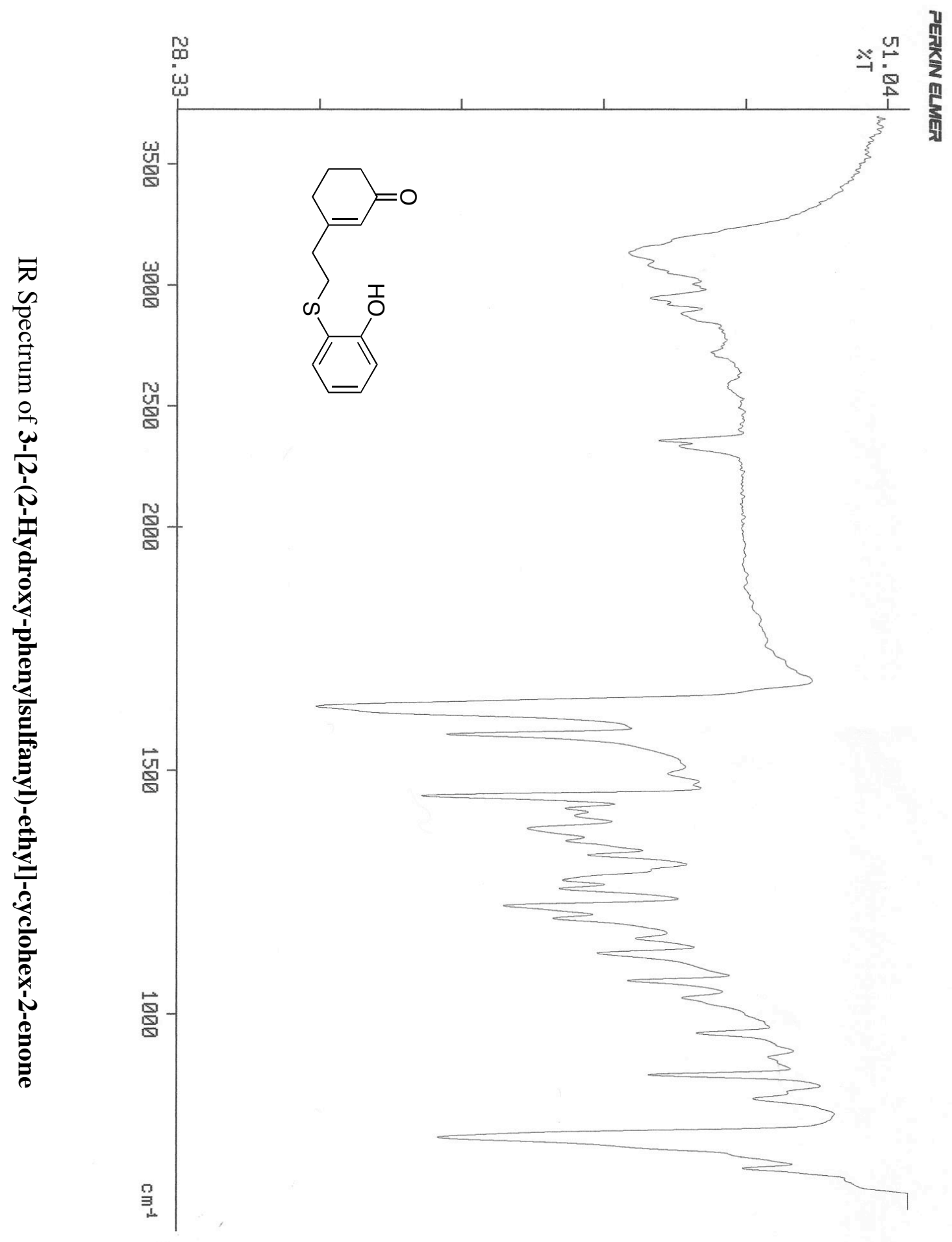

S-76 

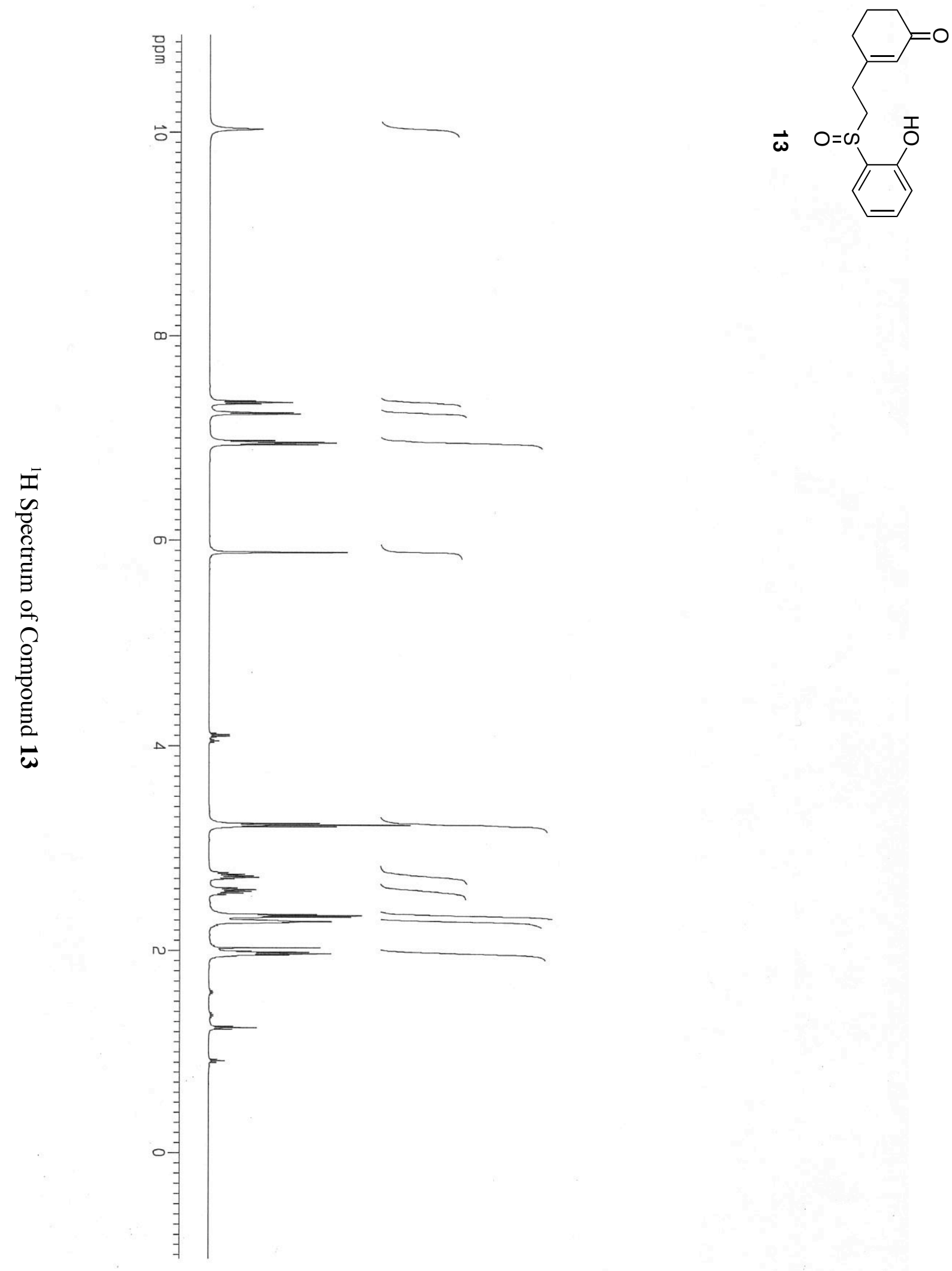

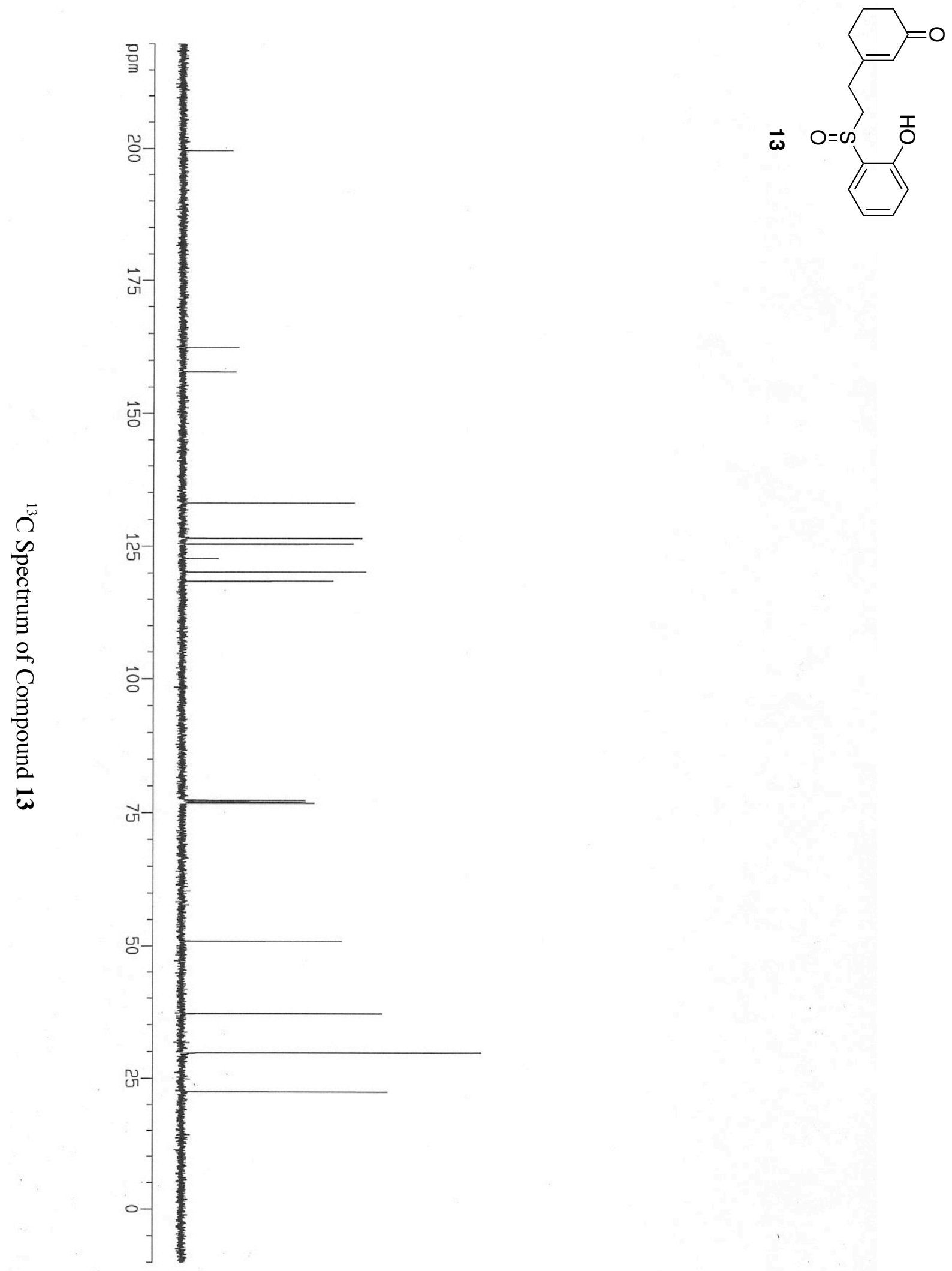


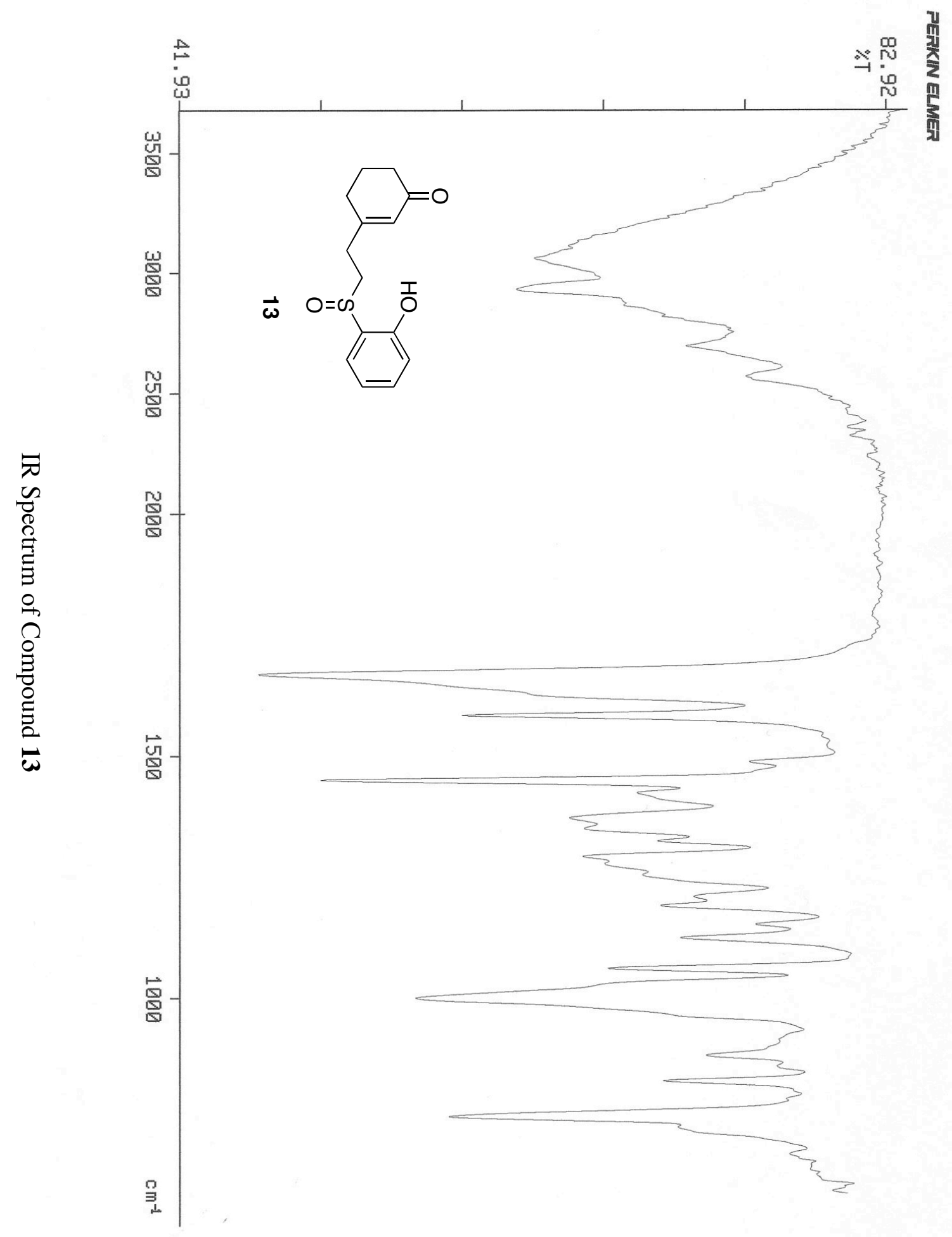




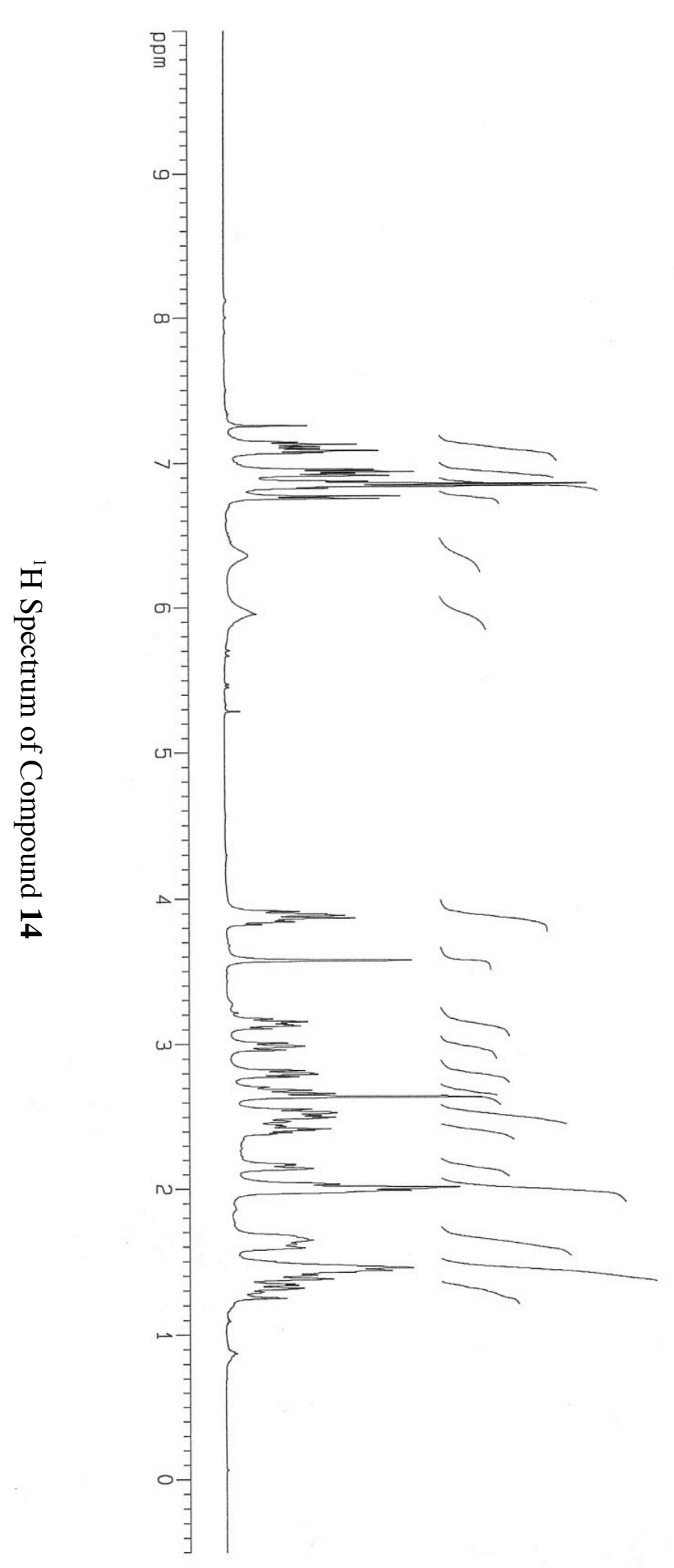




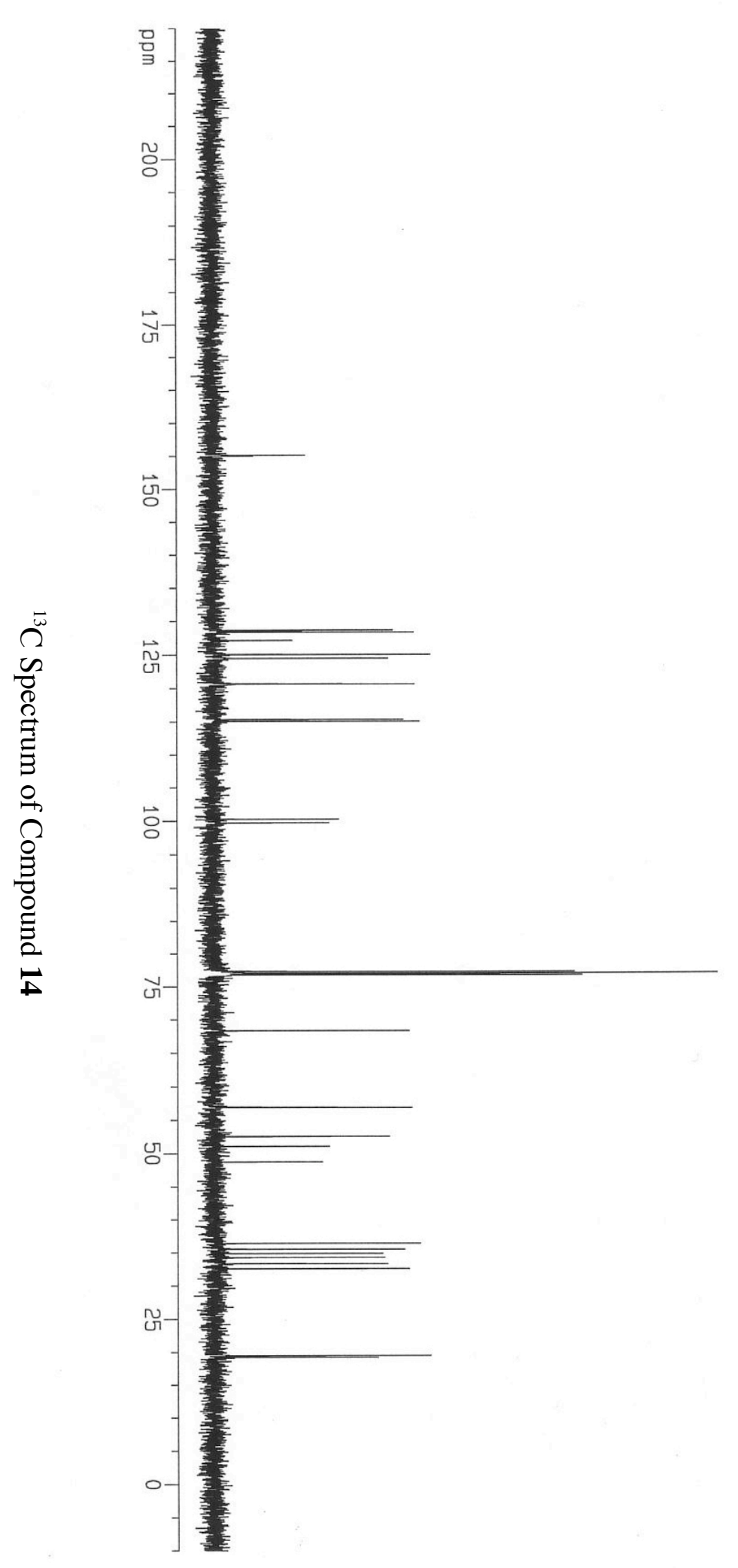




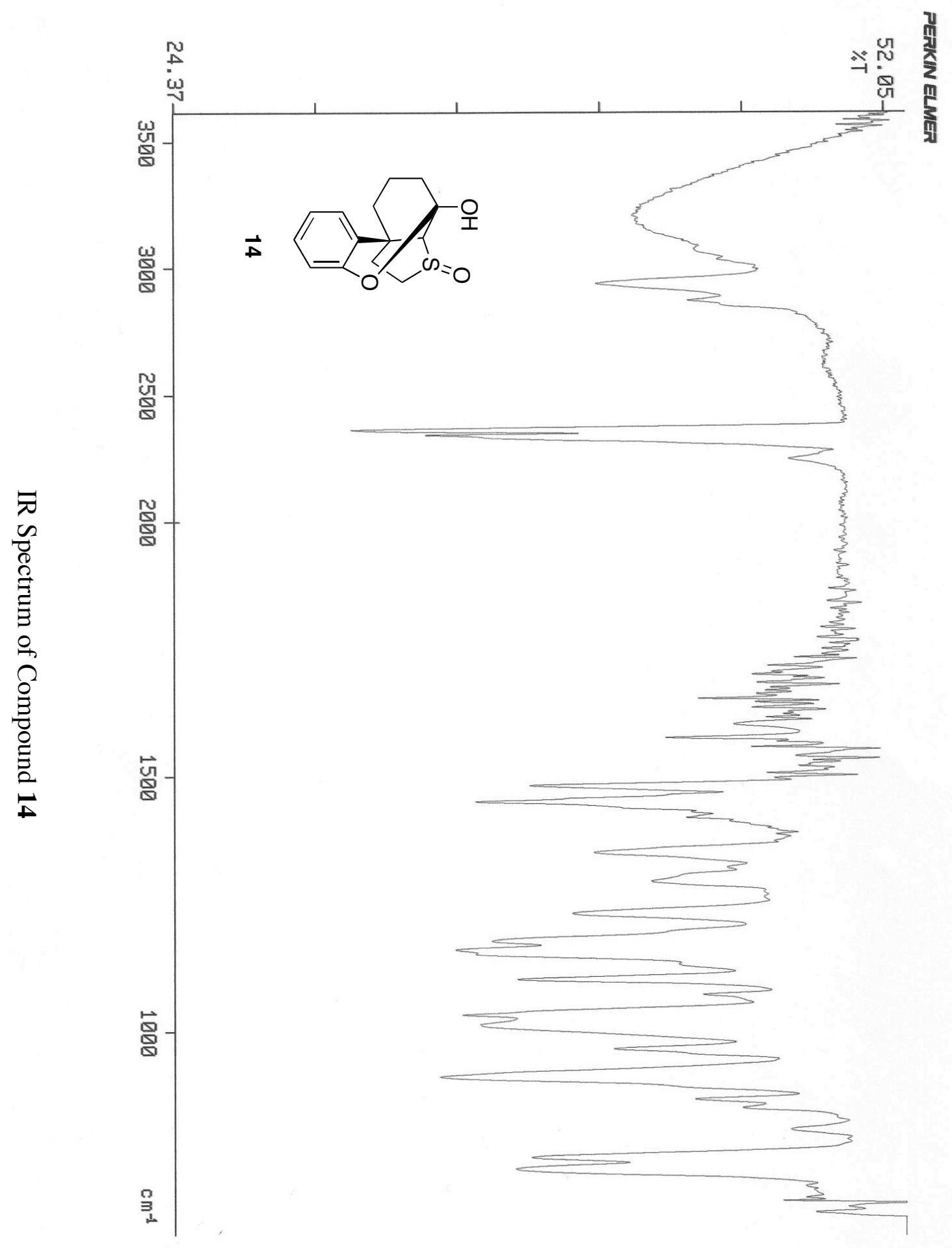

S-82 


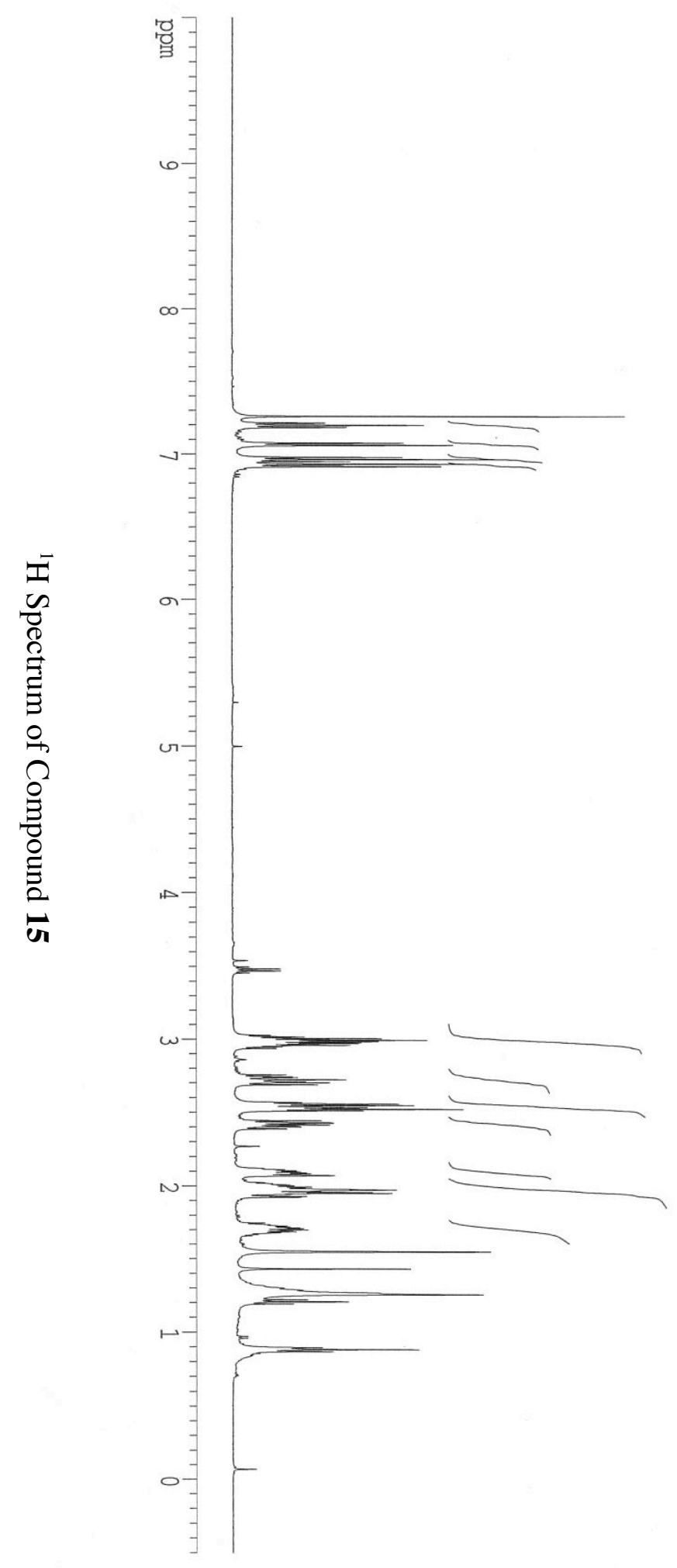




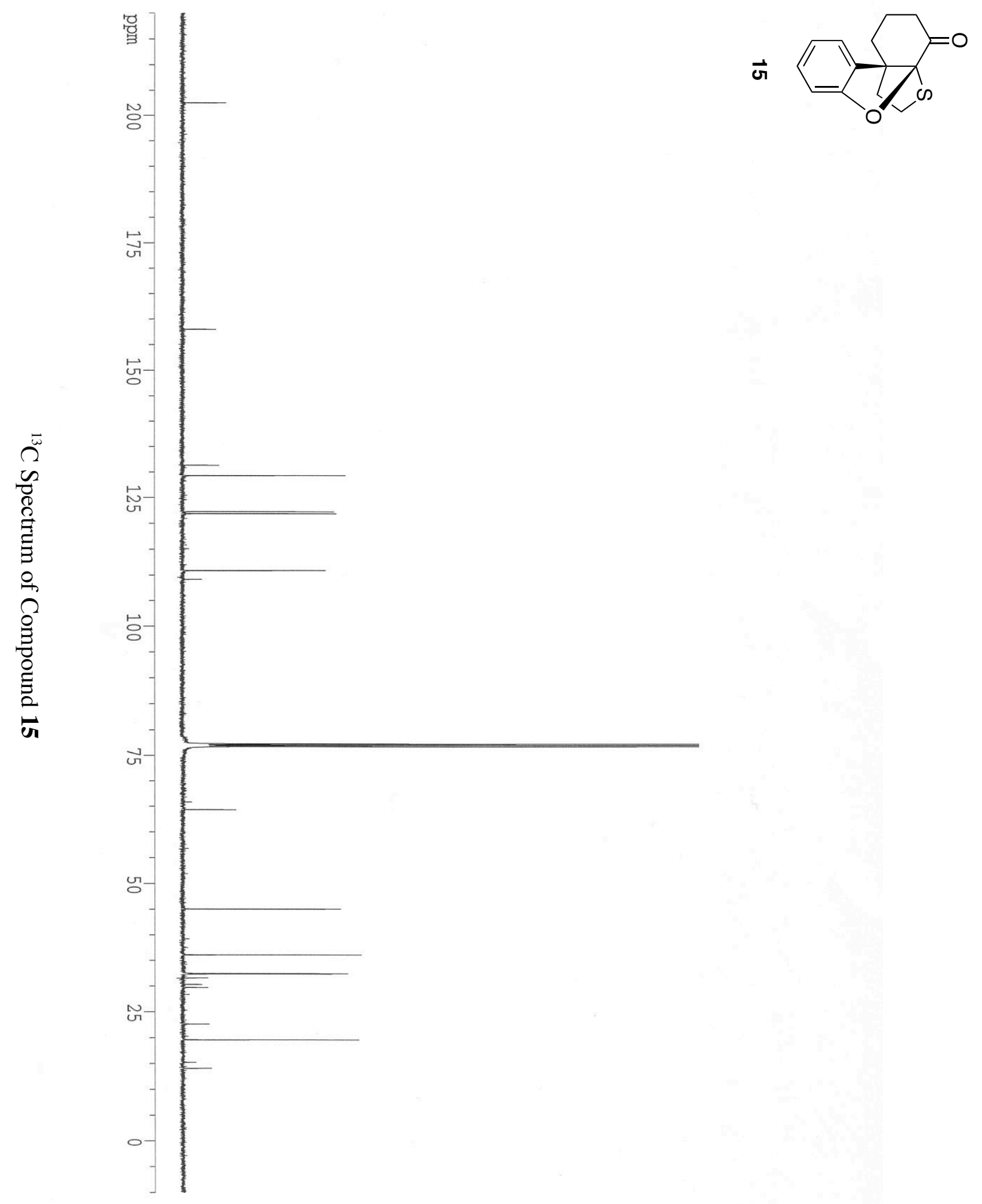




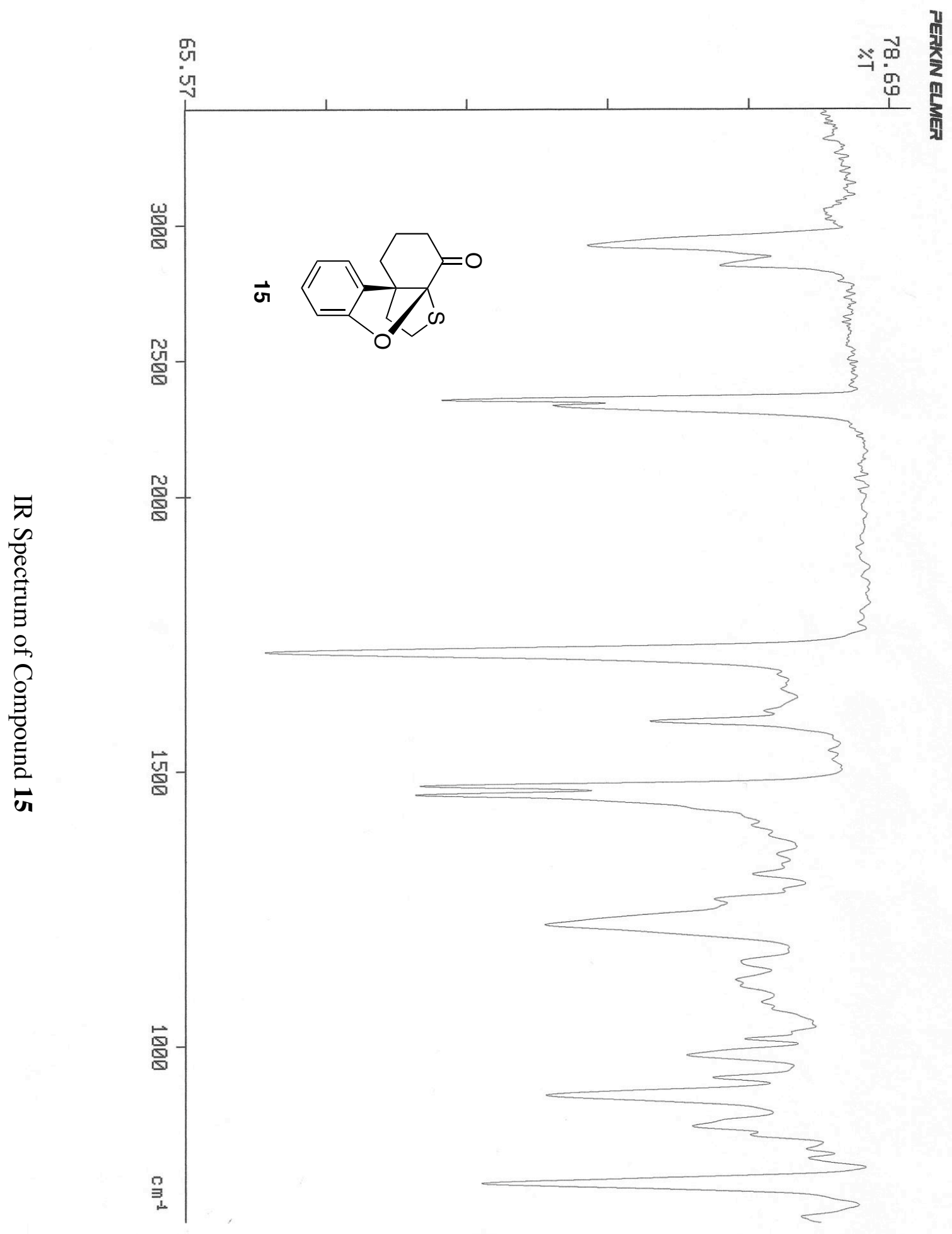




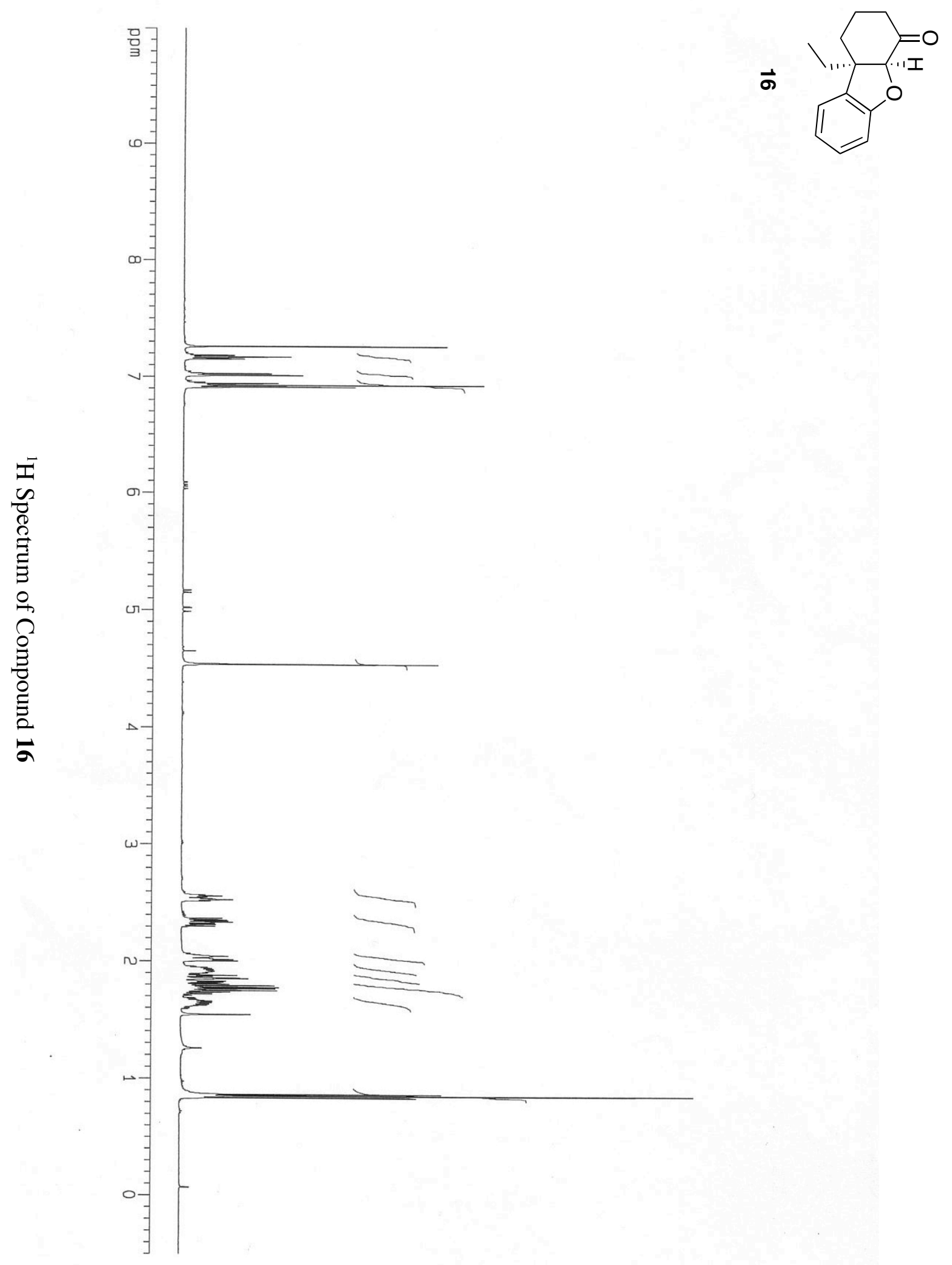




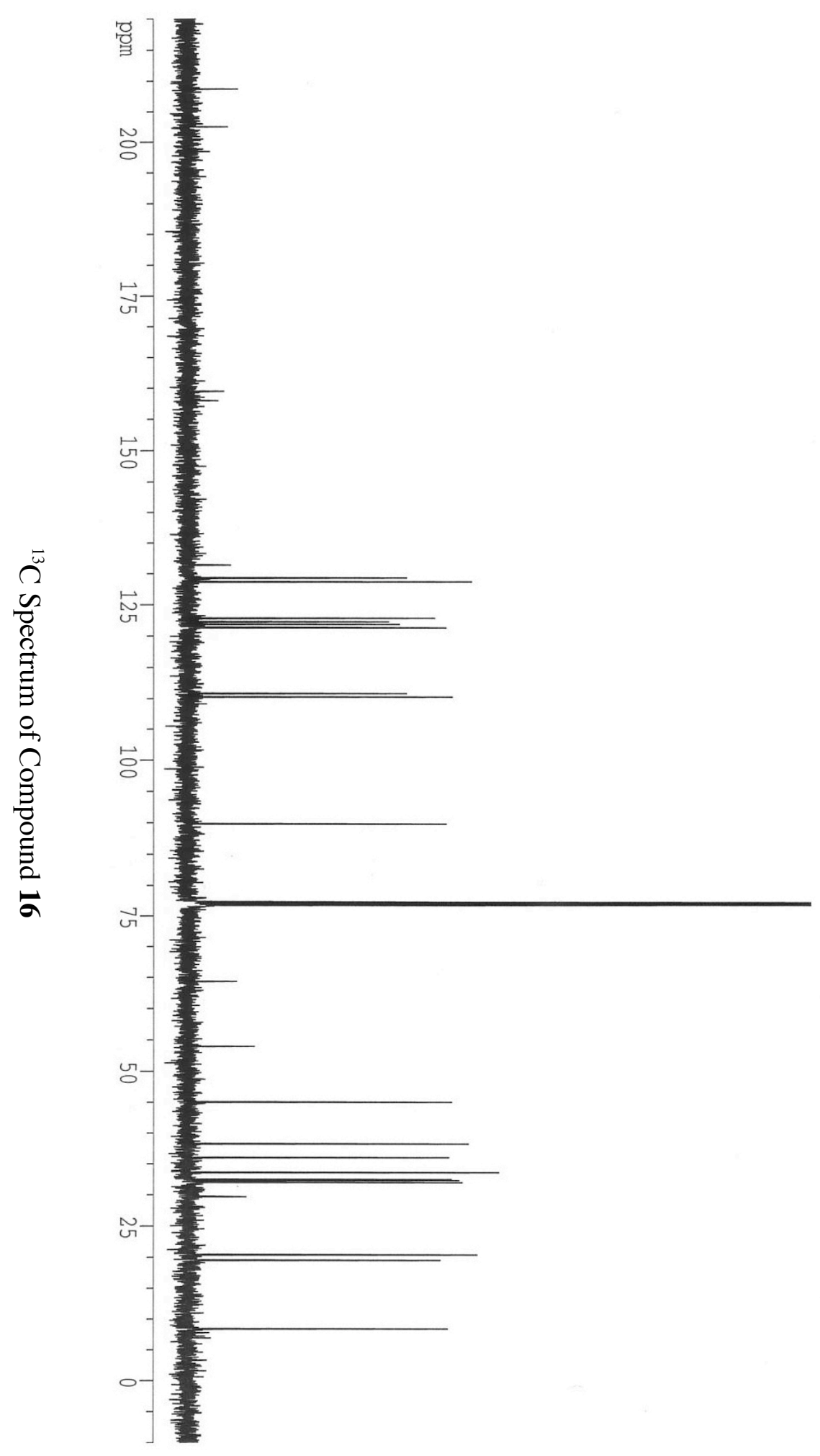

$=0$ 


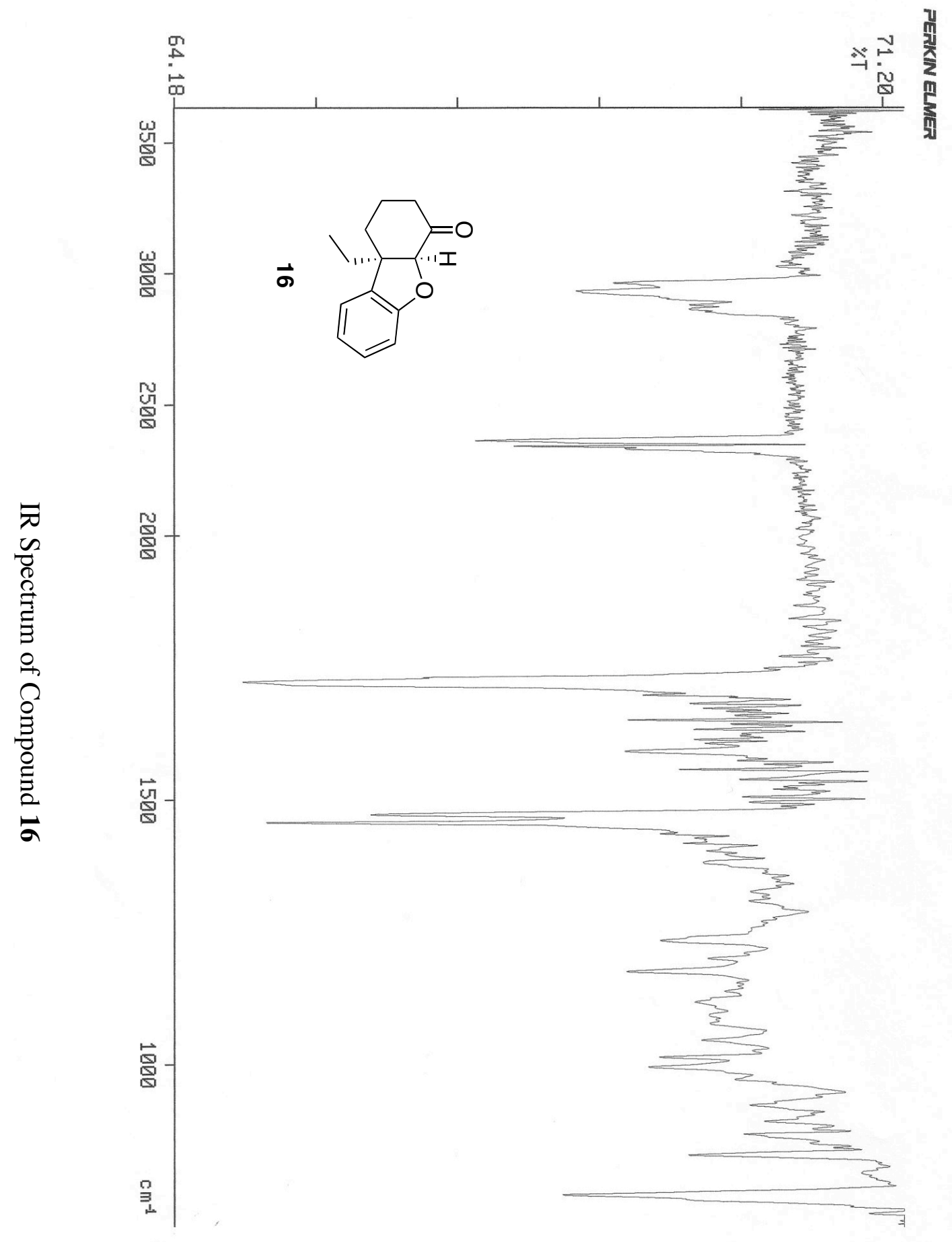



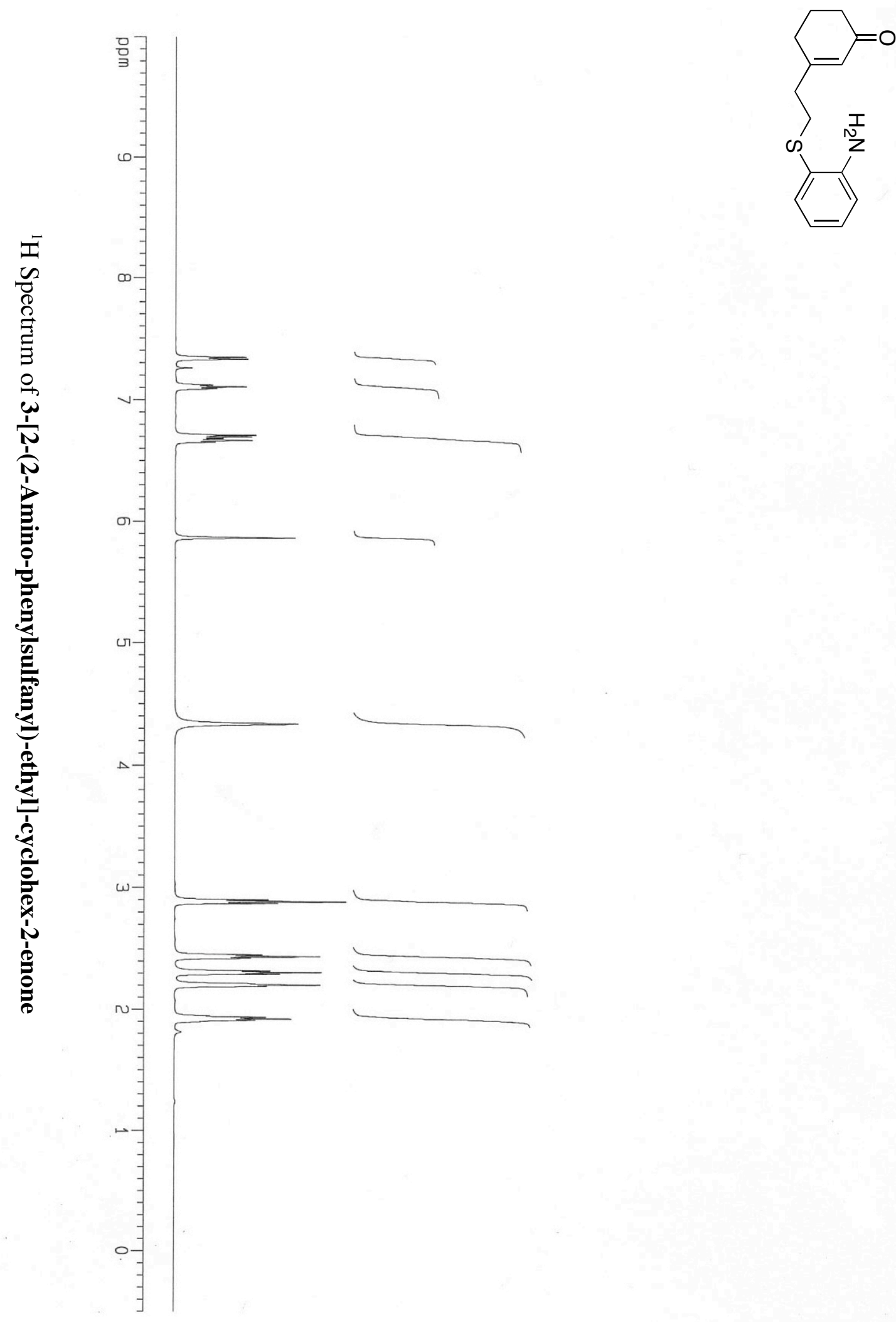

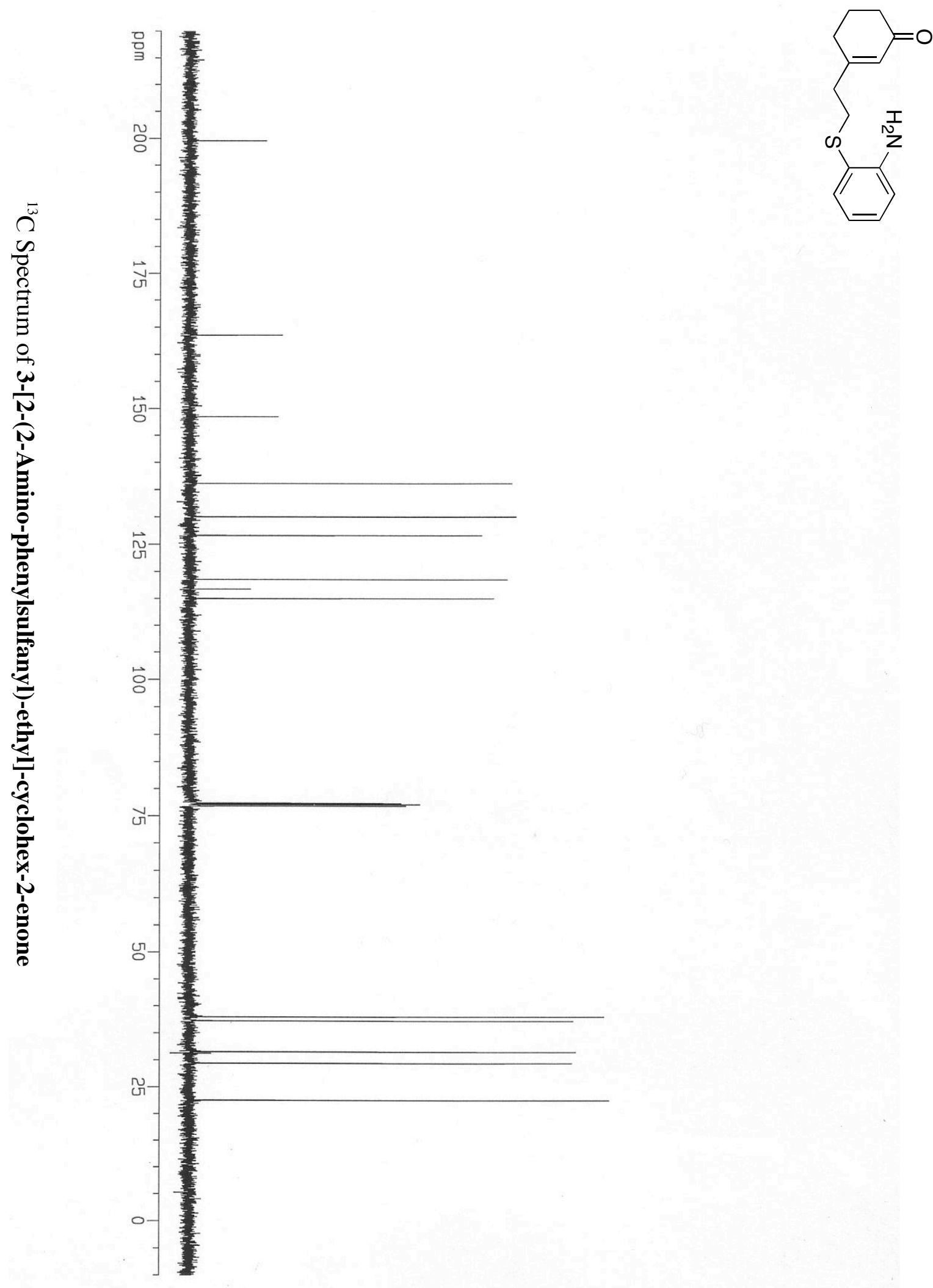

S-90 


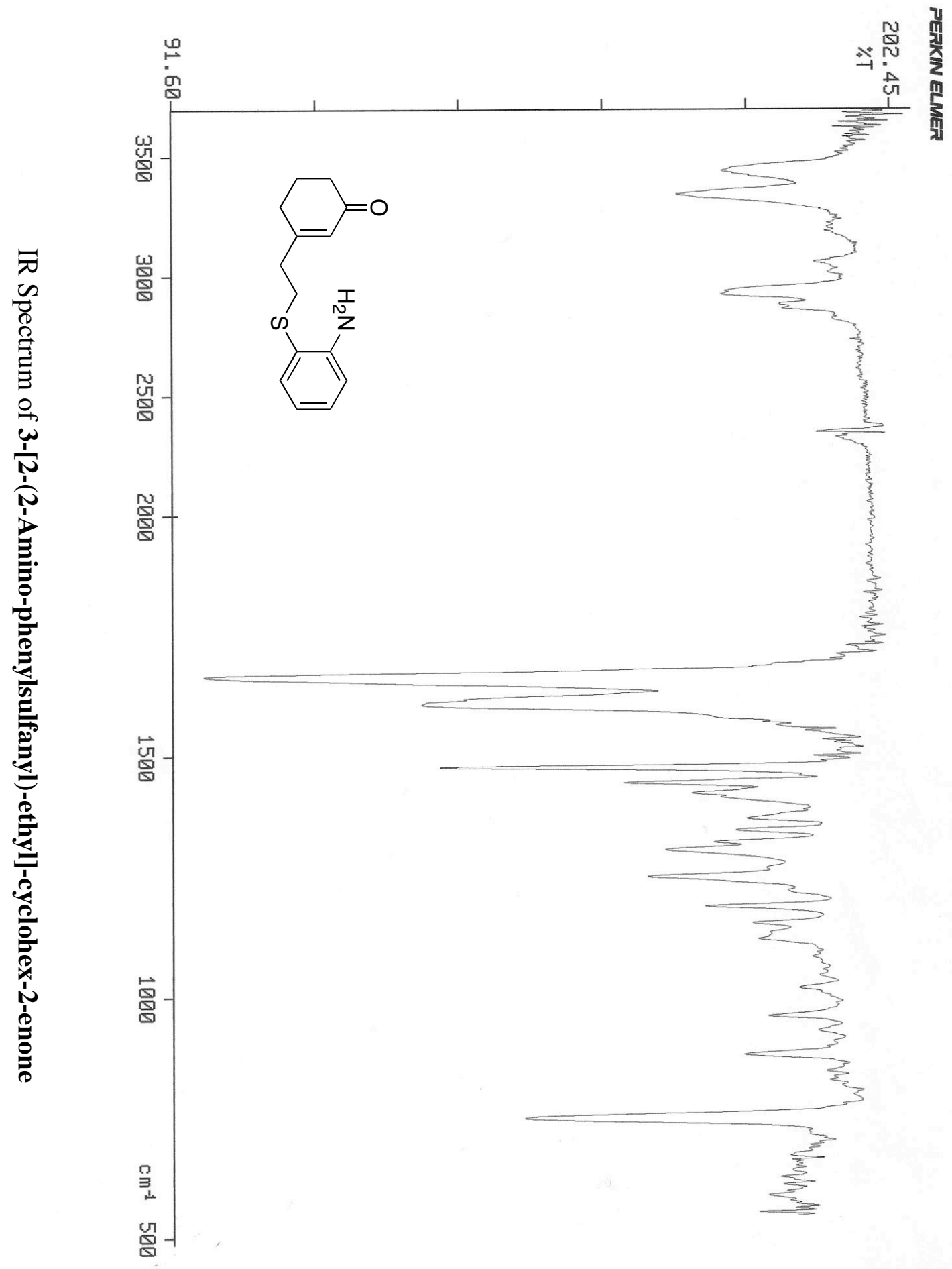

S-91 

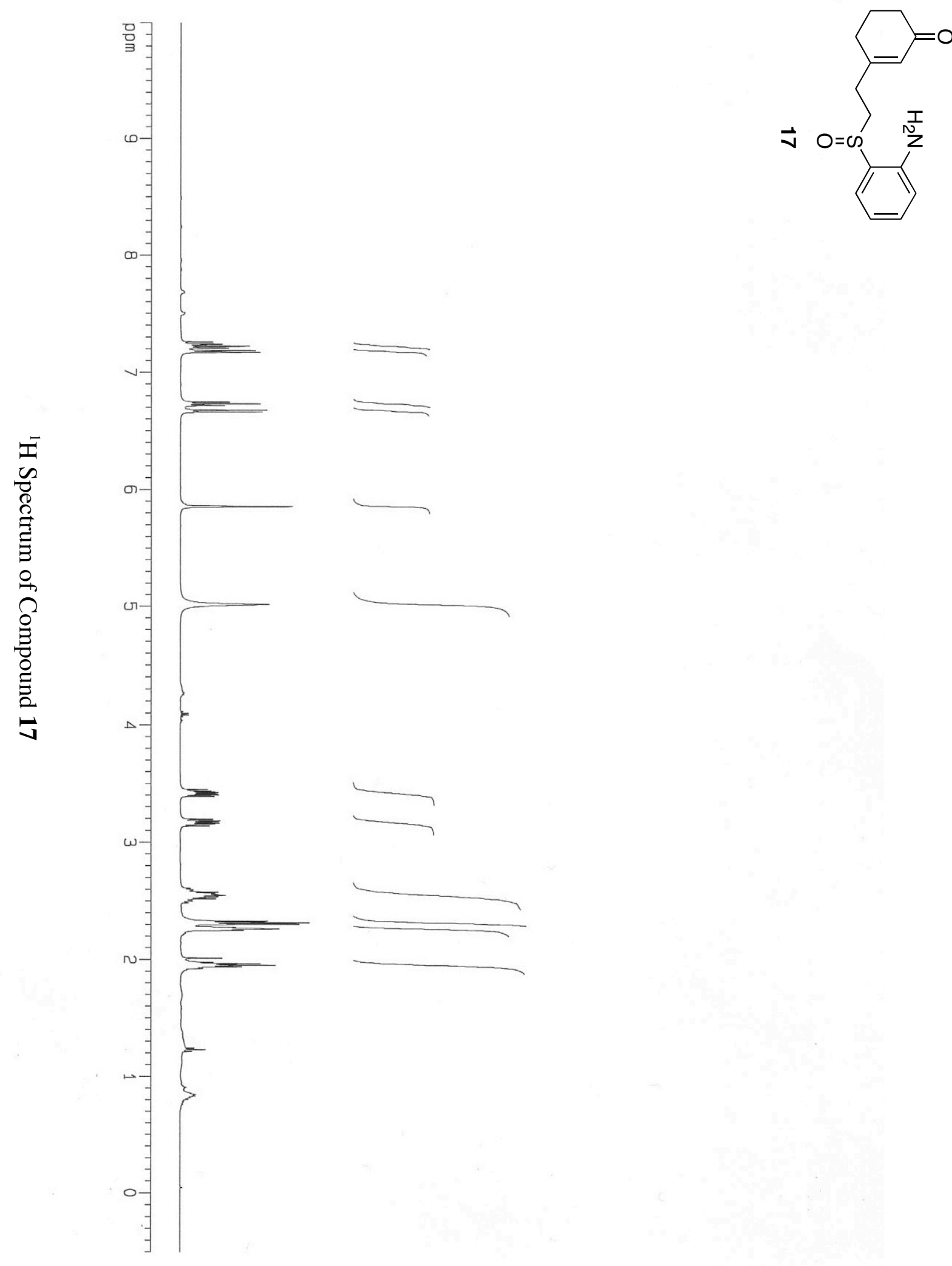

S-92 

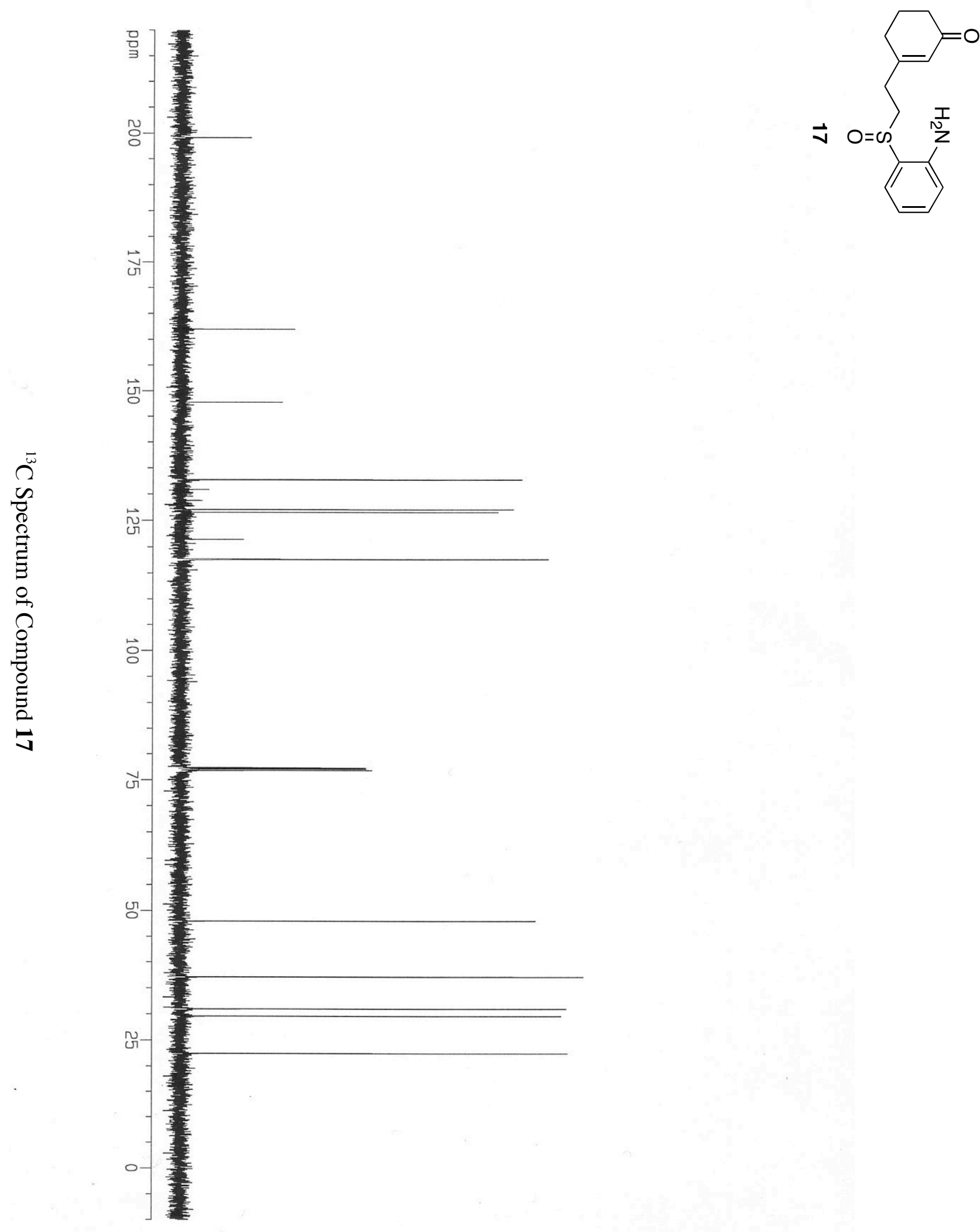

S-93 


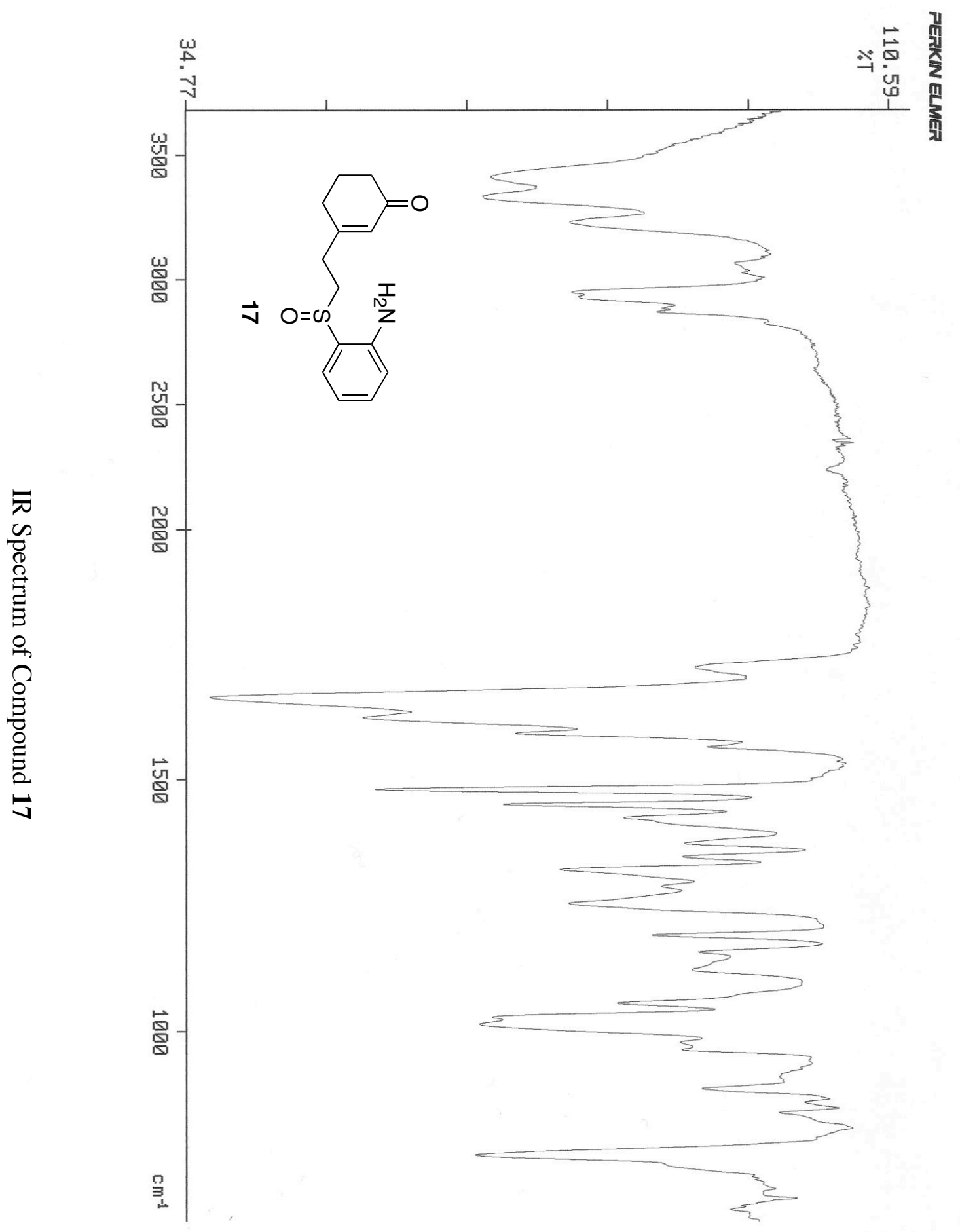




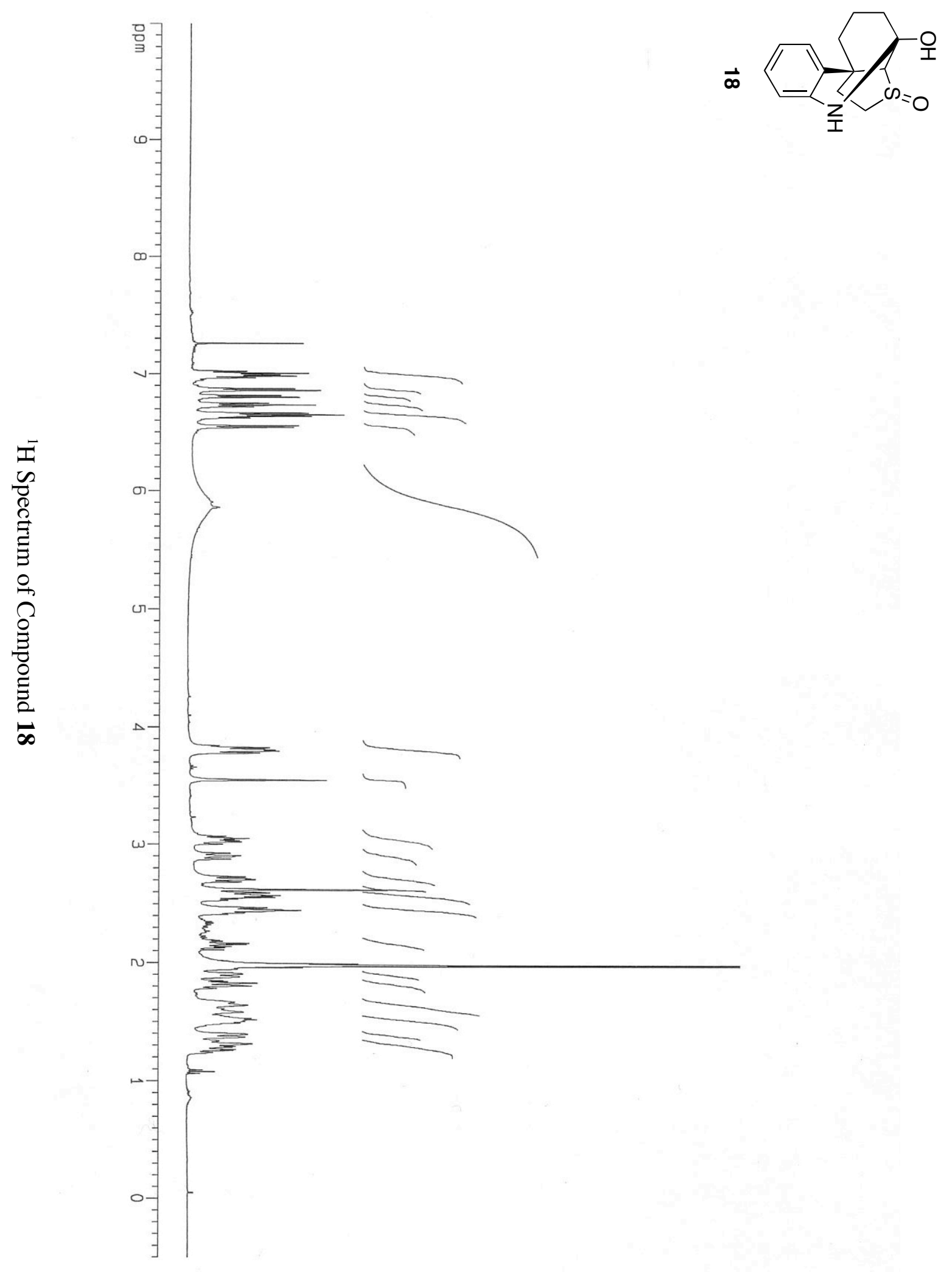

S-95 


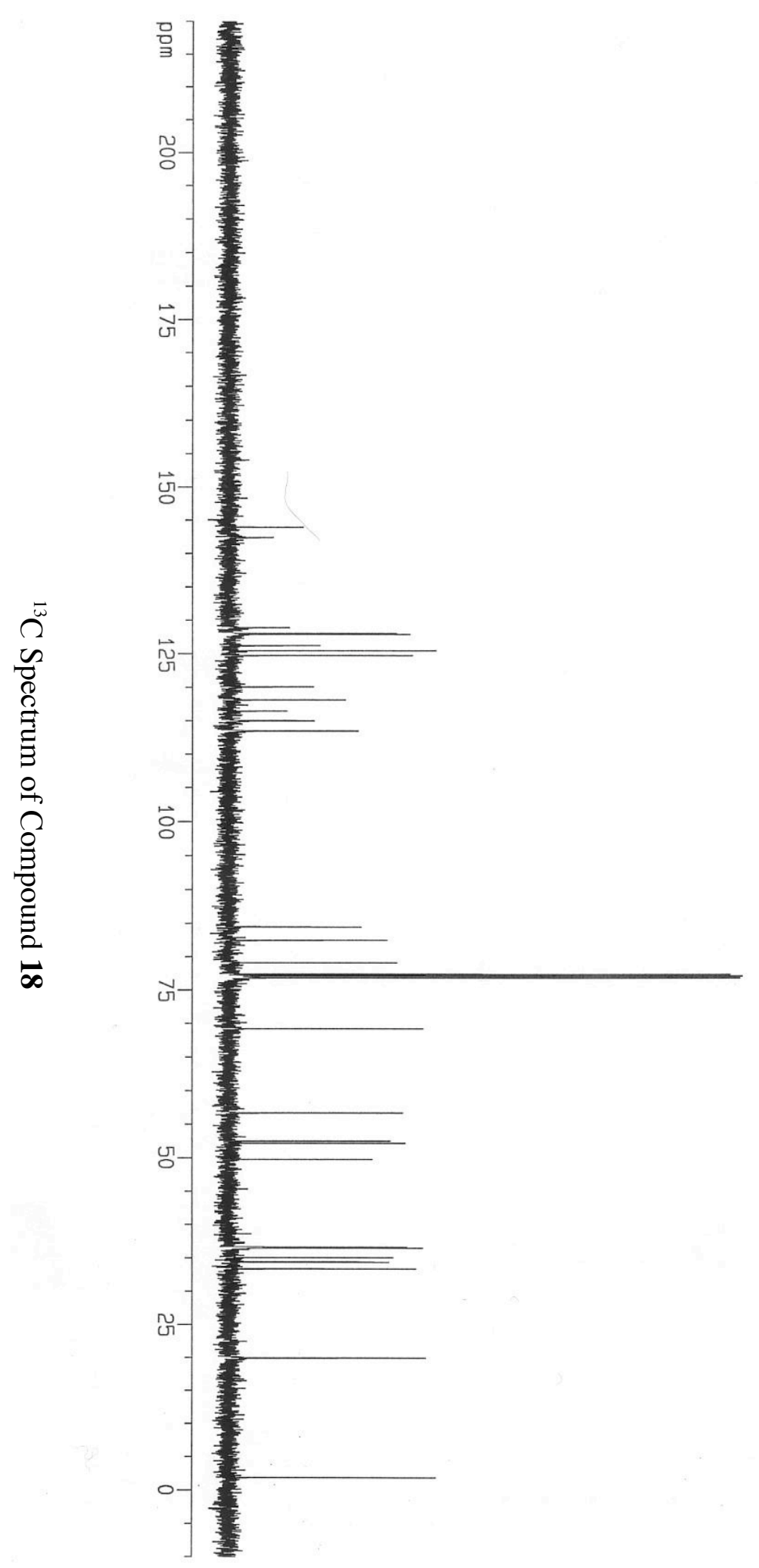




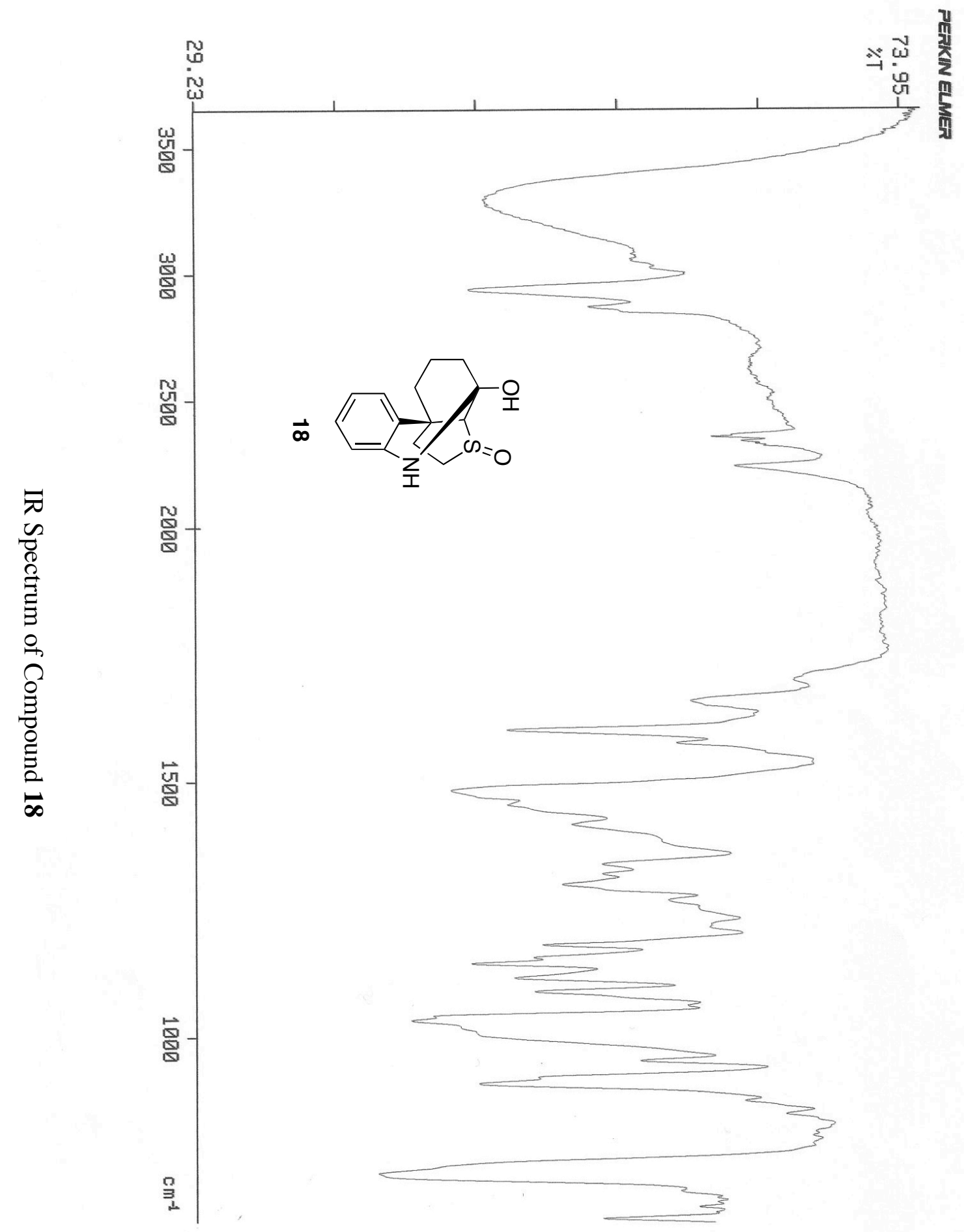



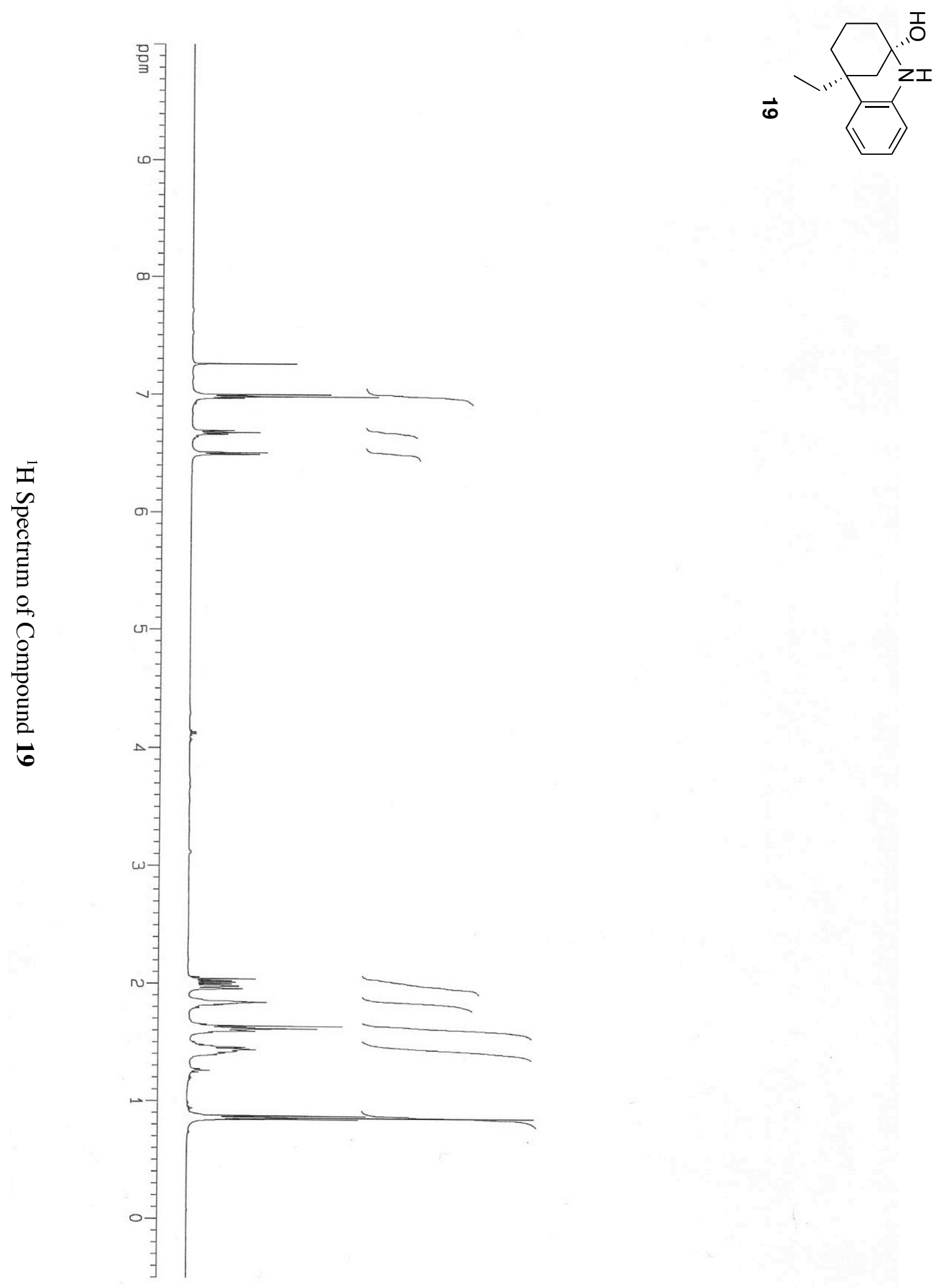


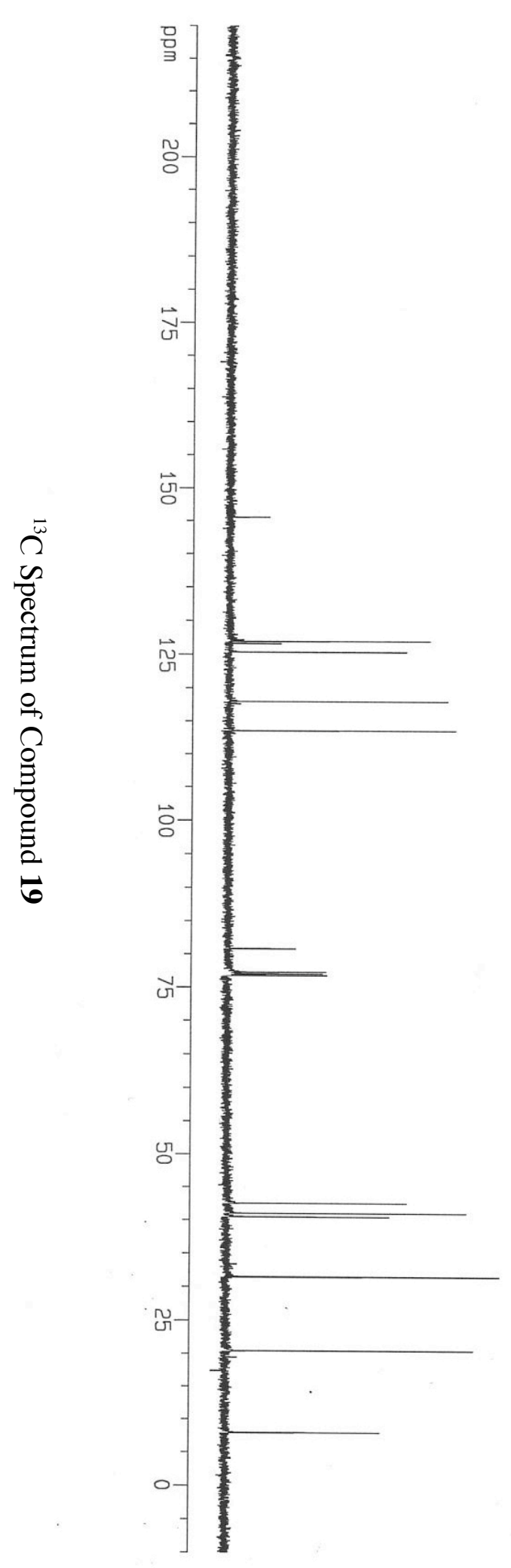




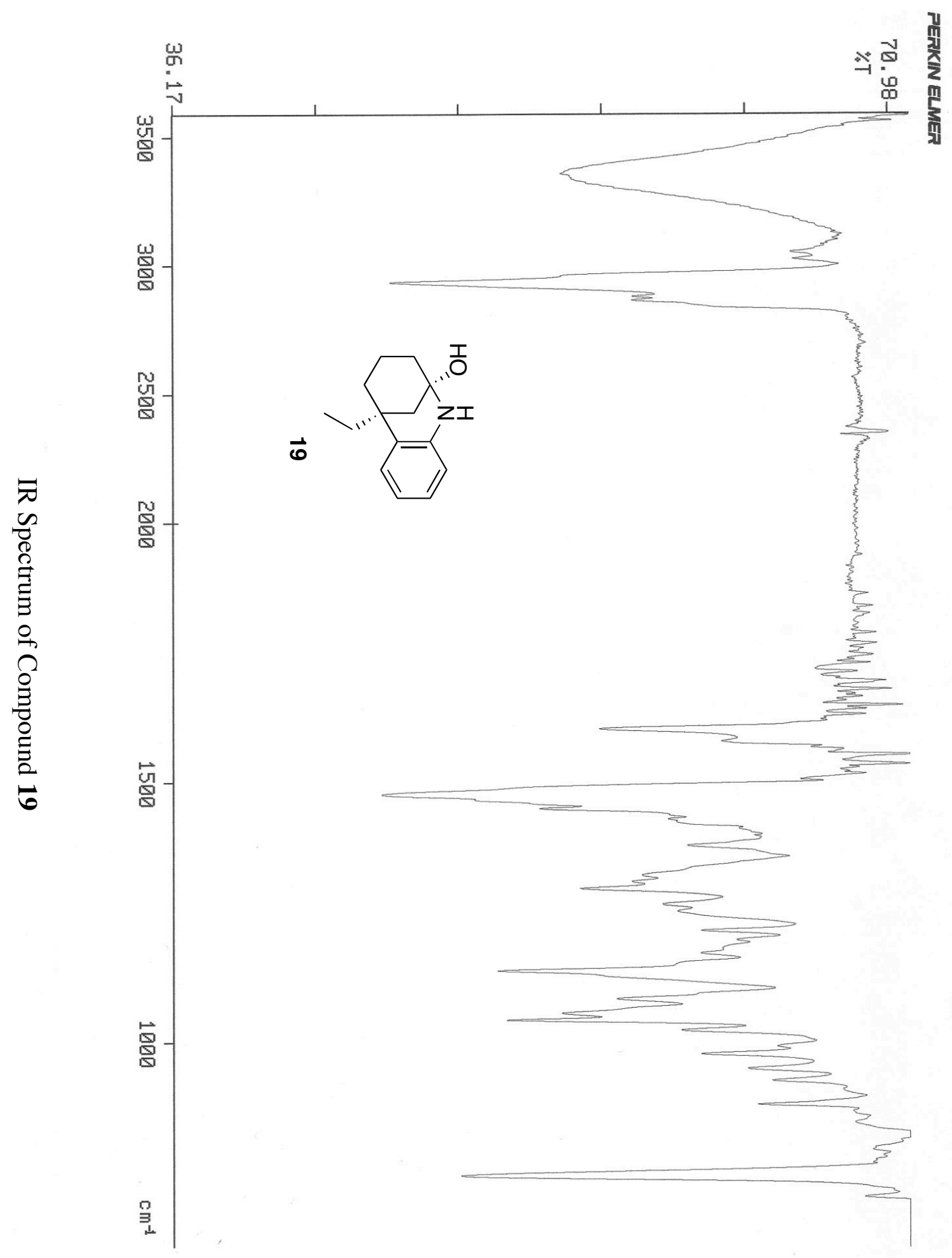




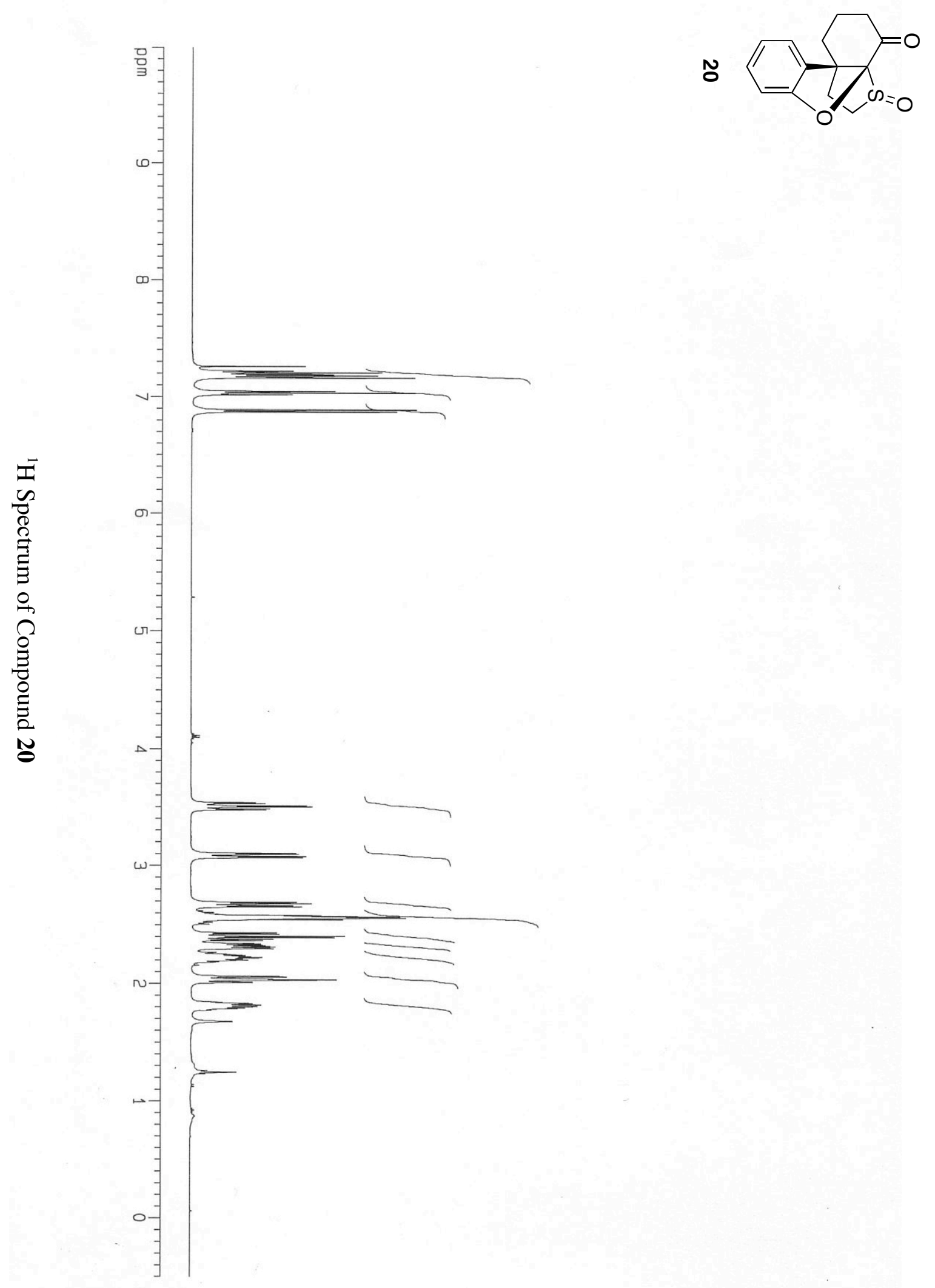

S-101 


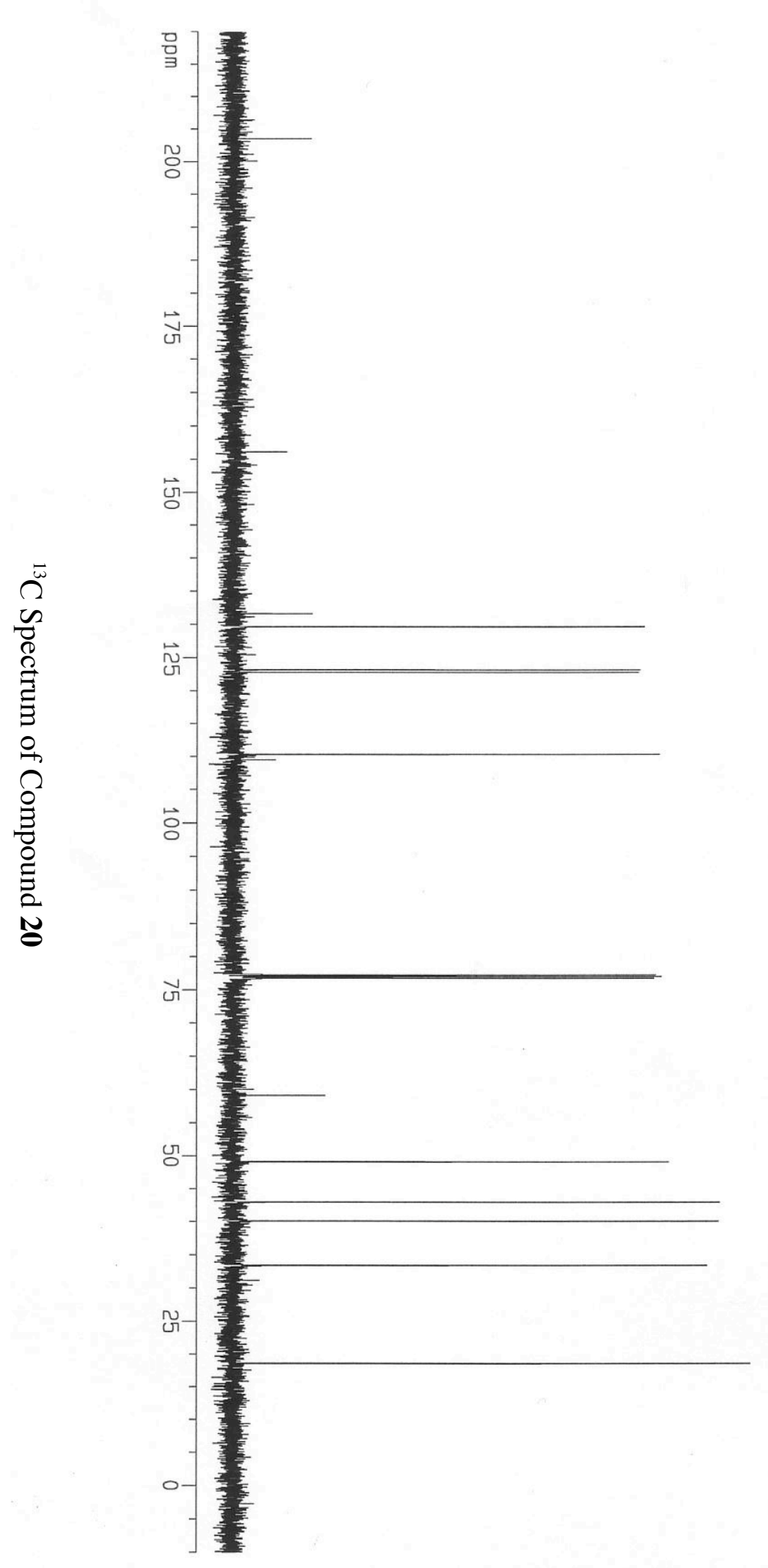




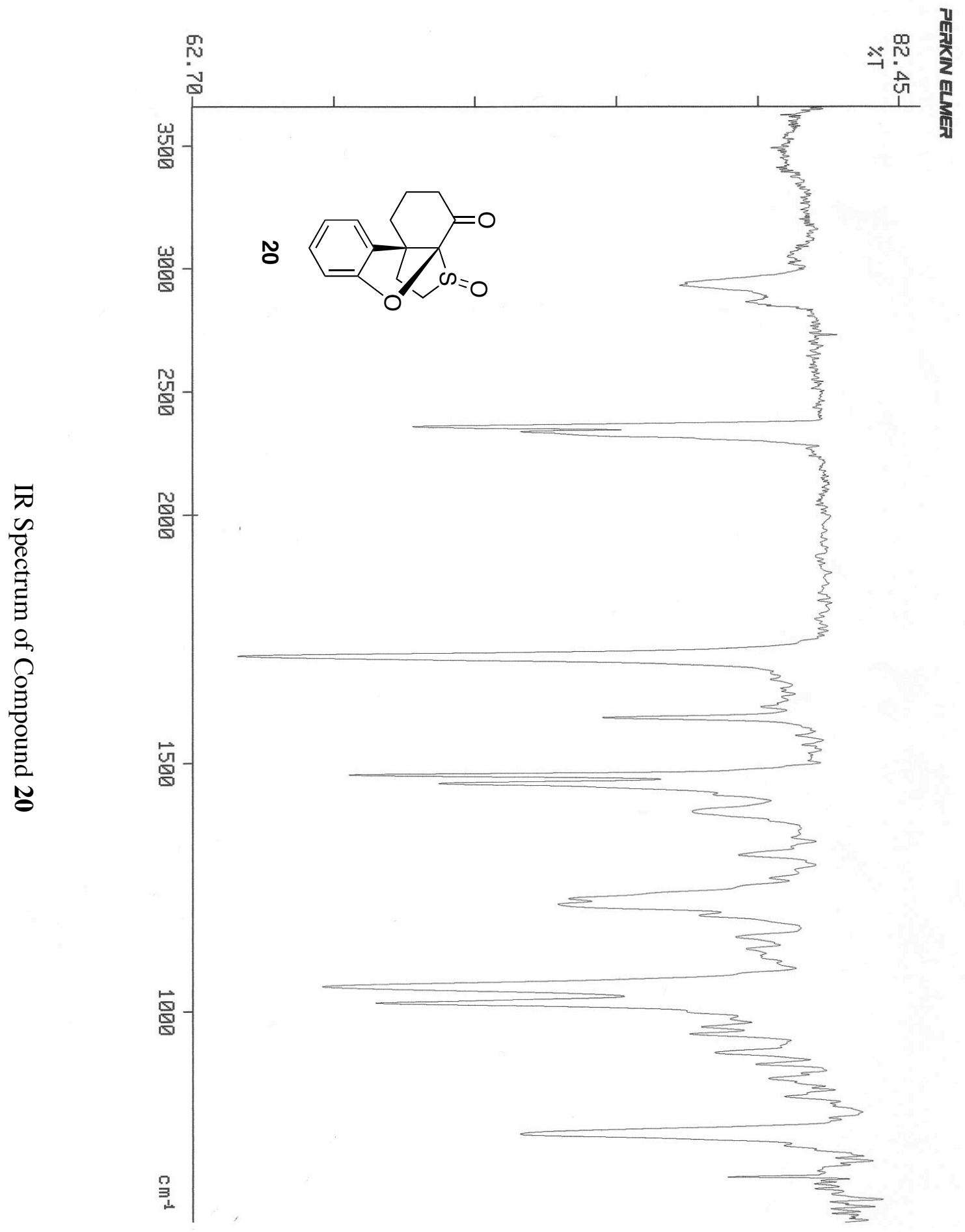

S-103 


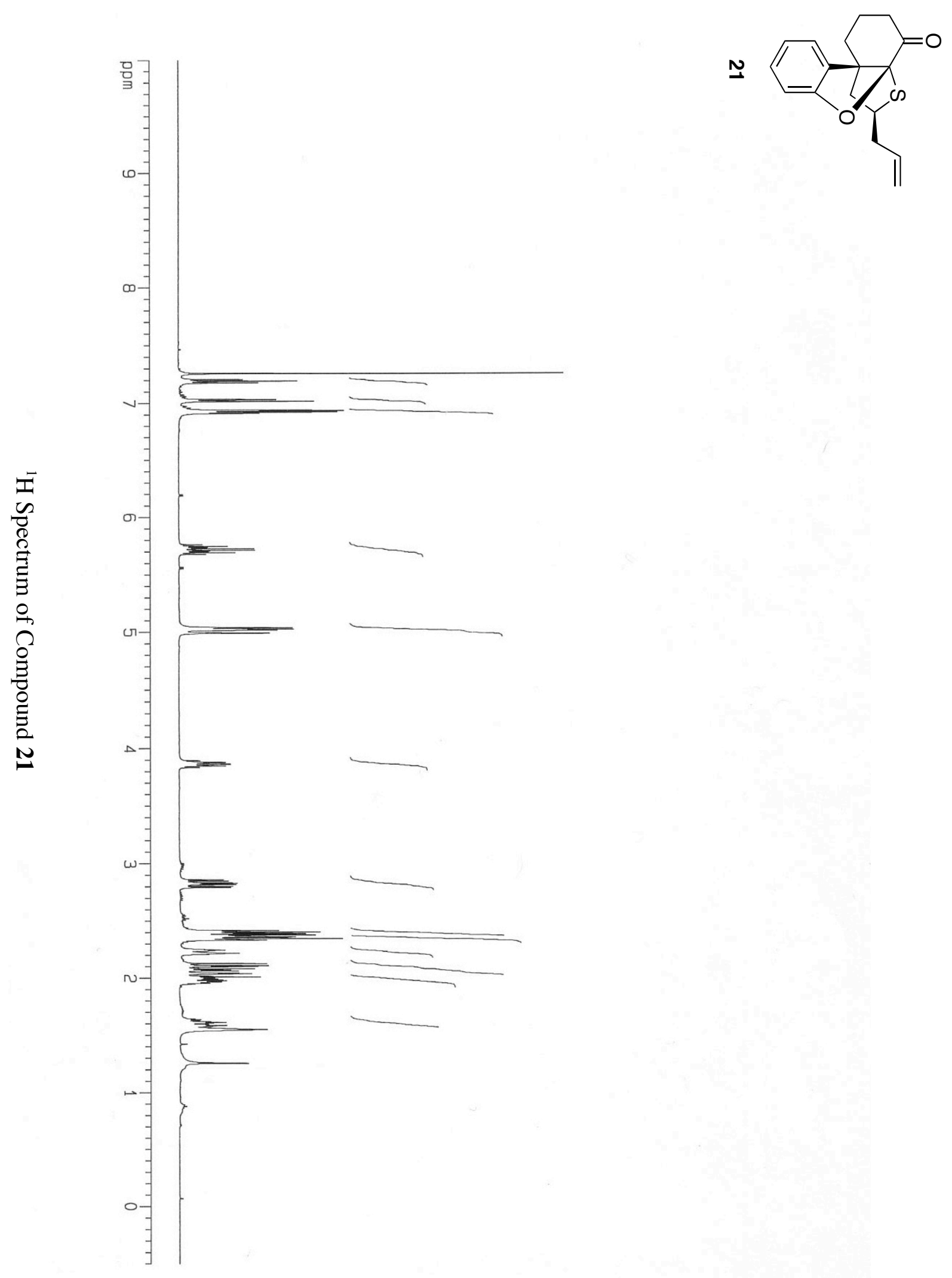




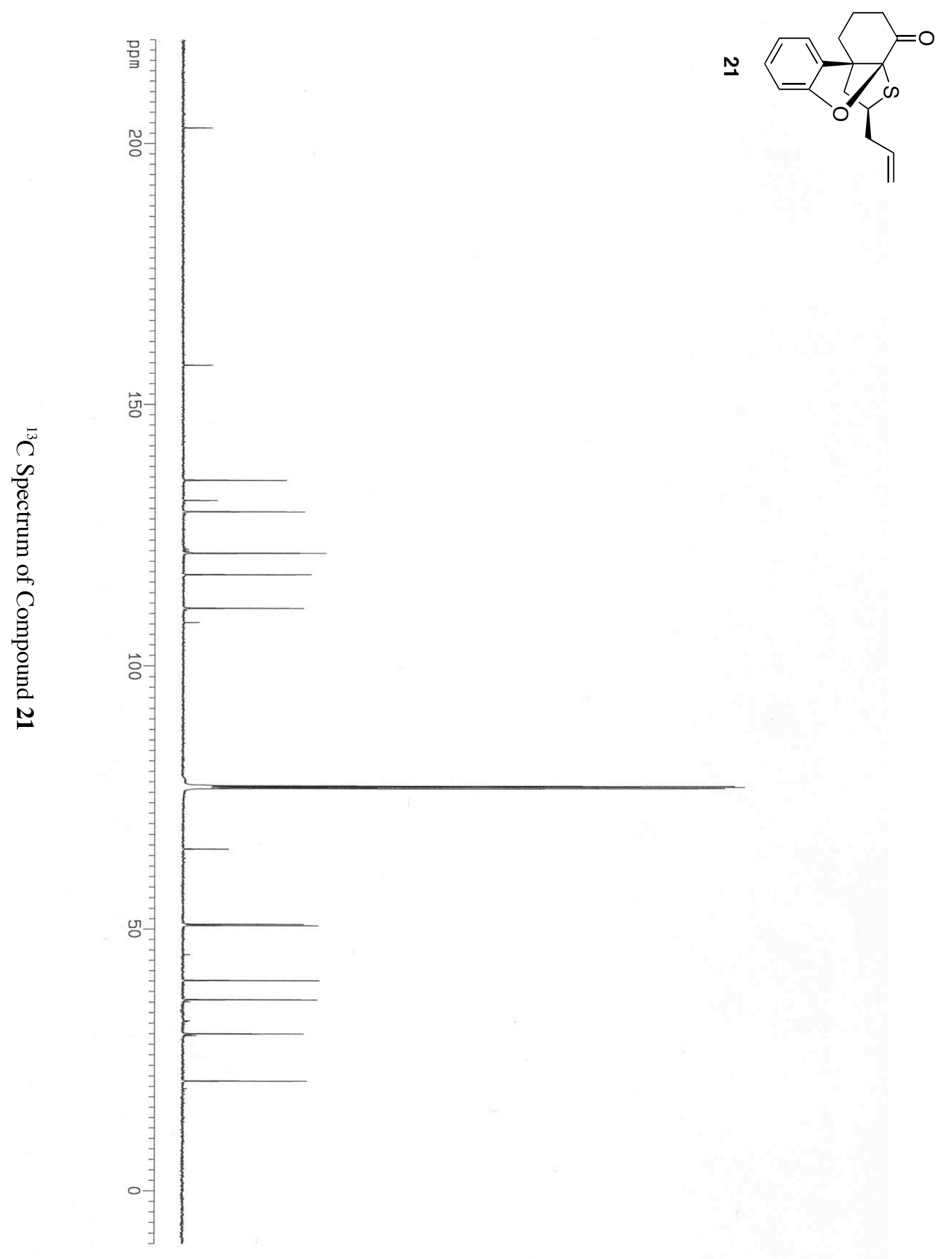




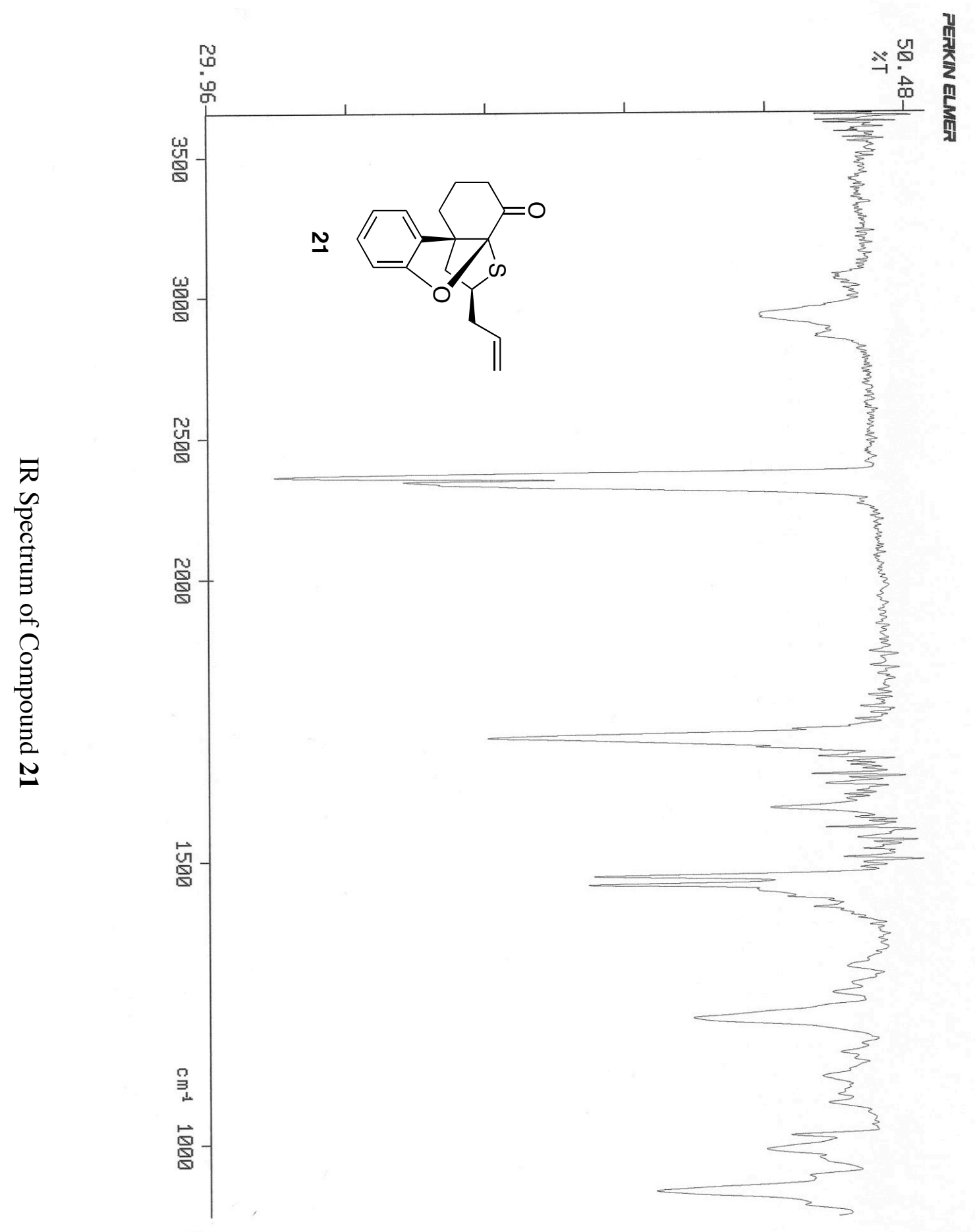

S-106 

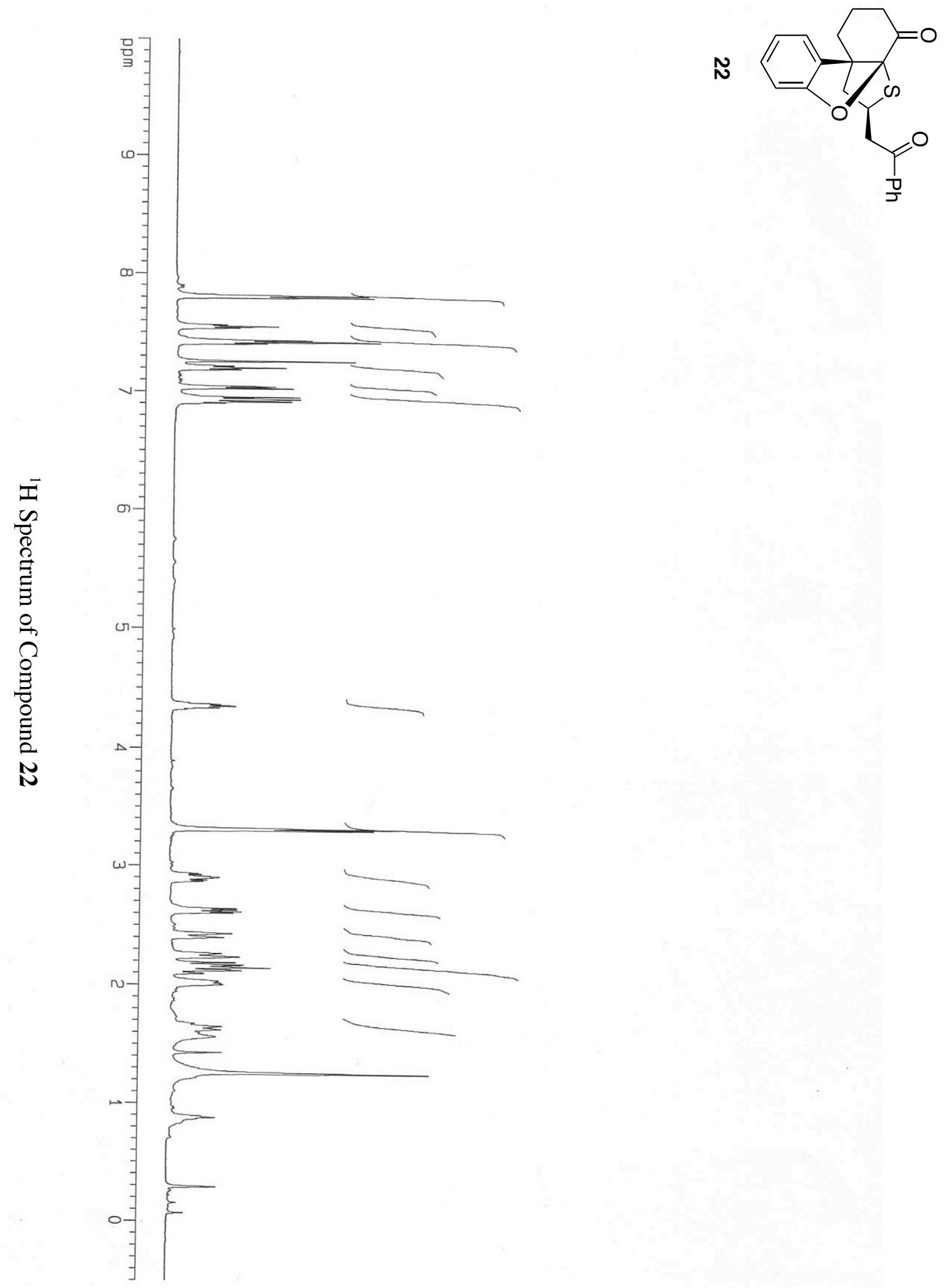


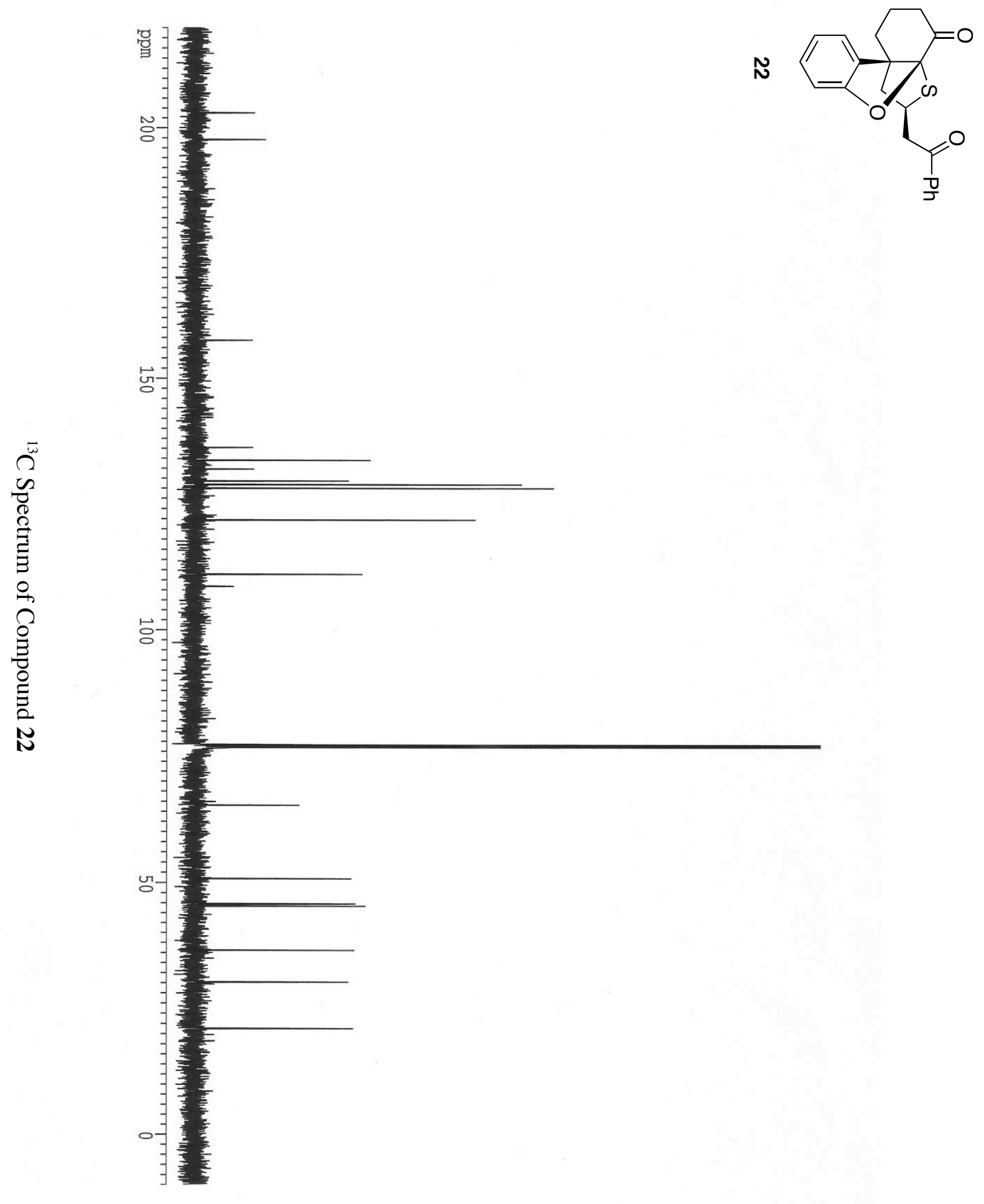




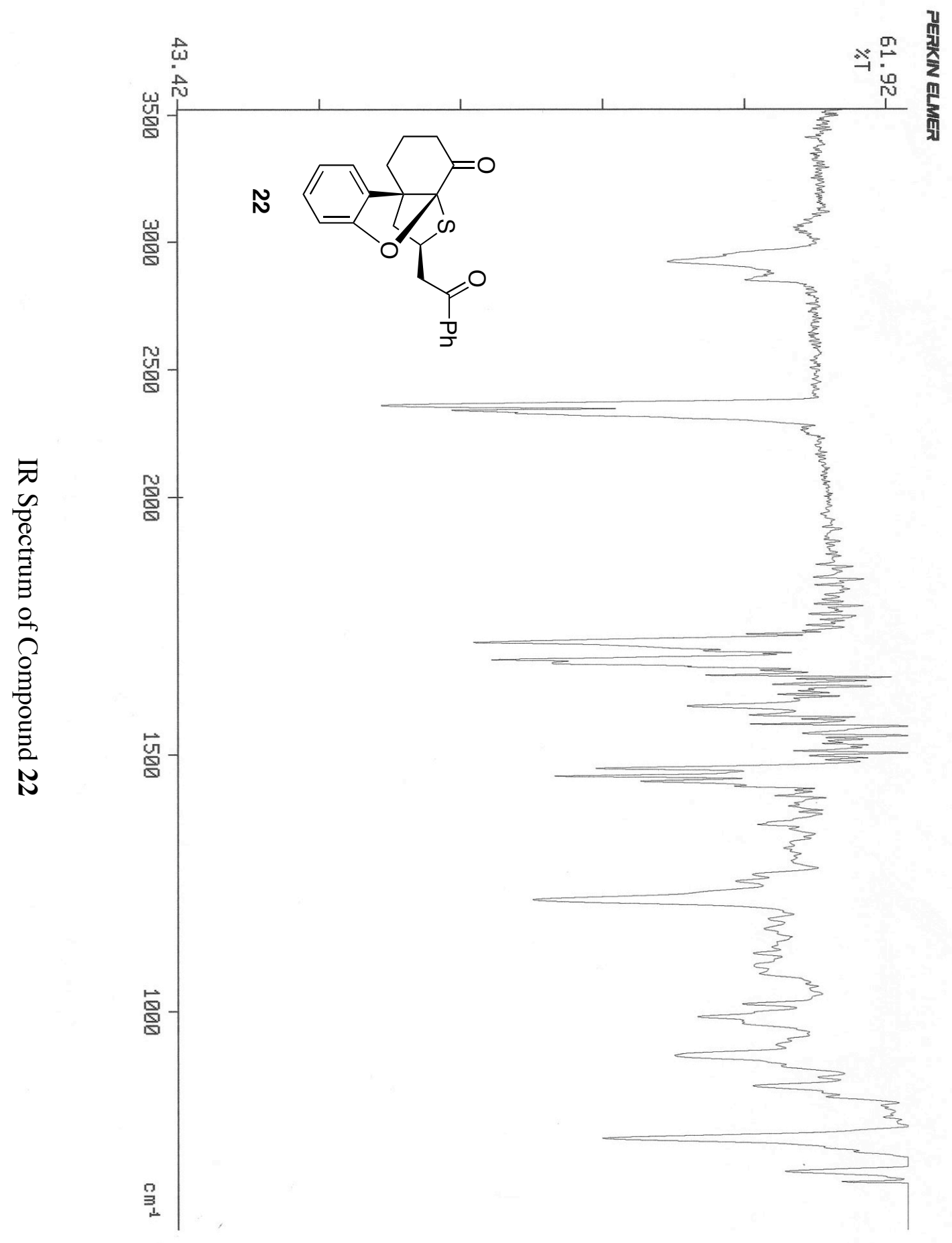

S-109 


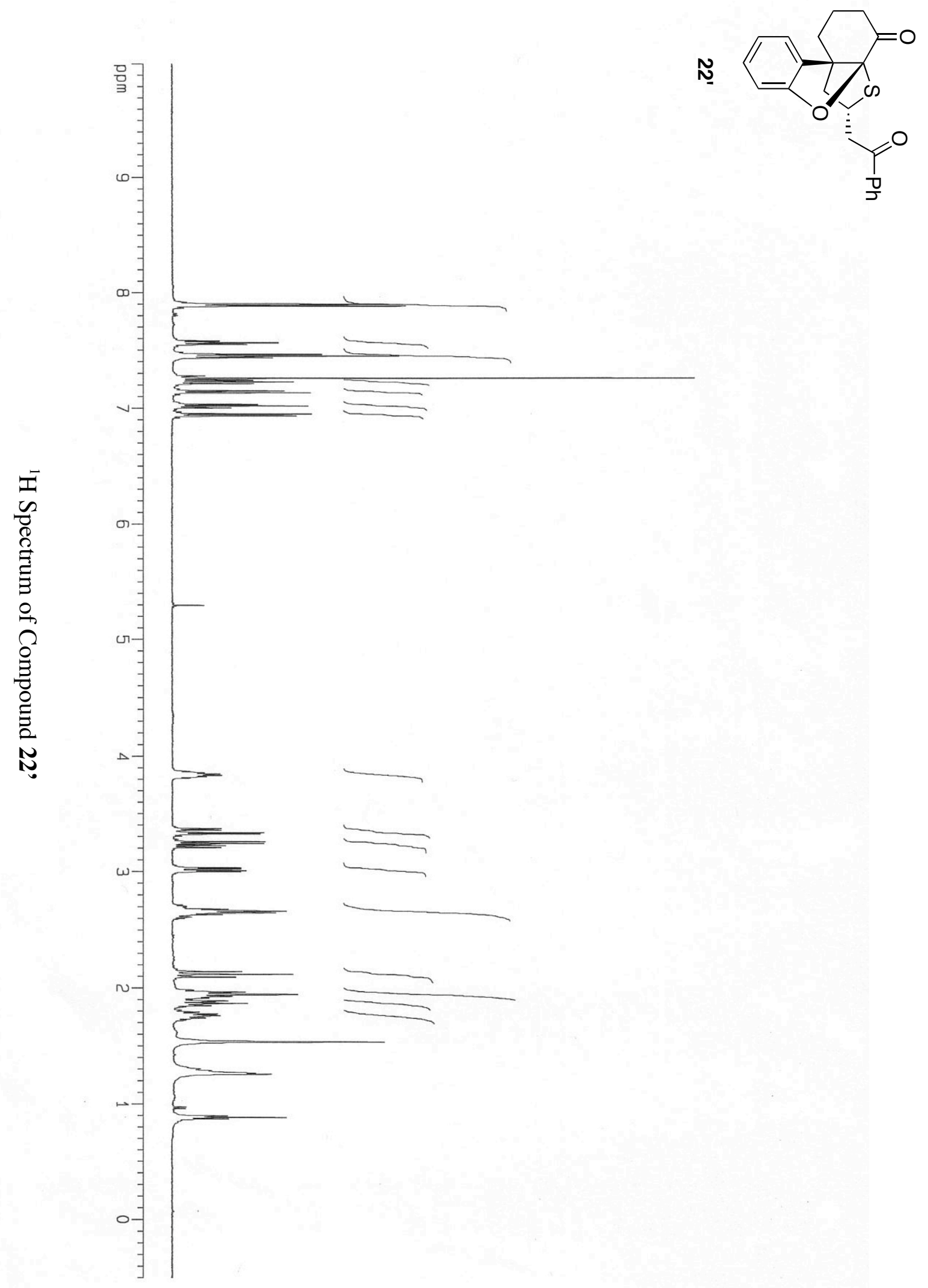

S-110 


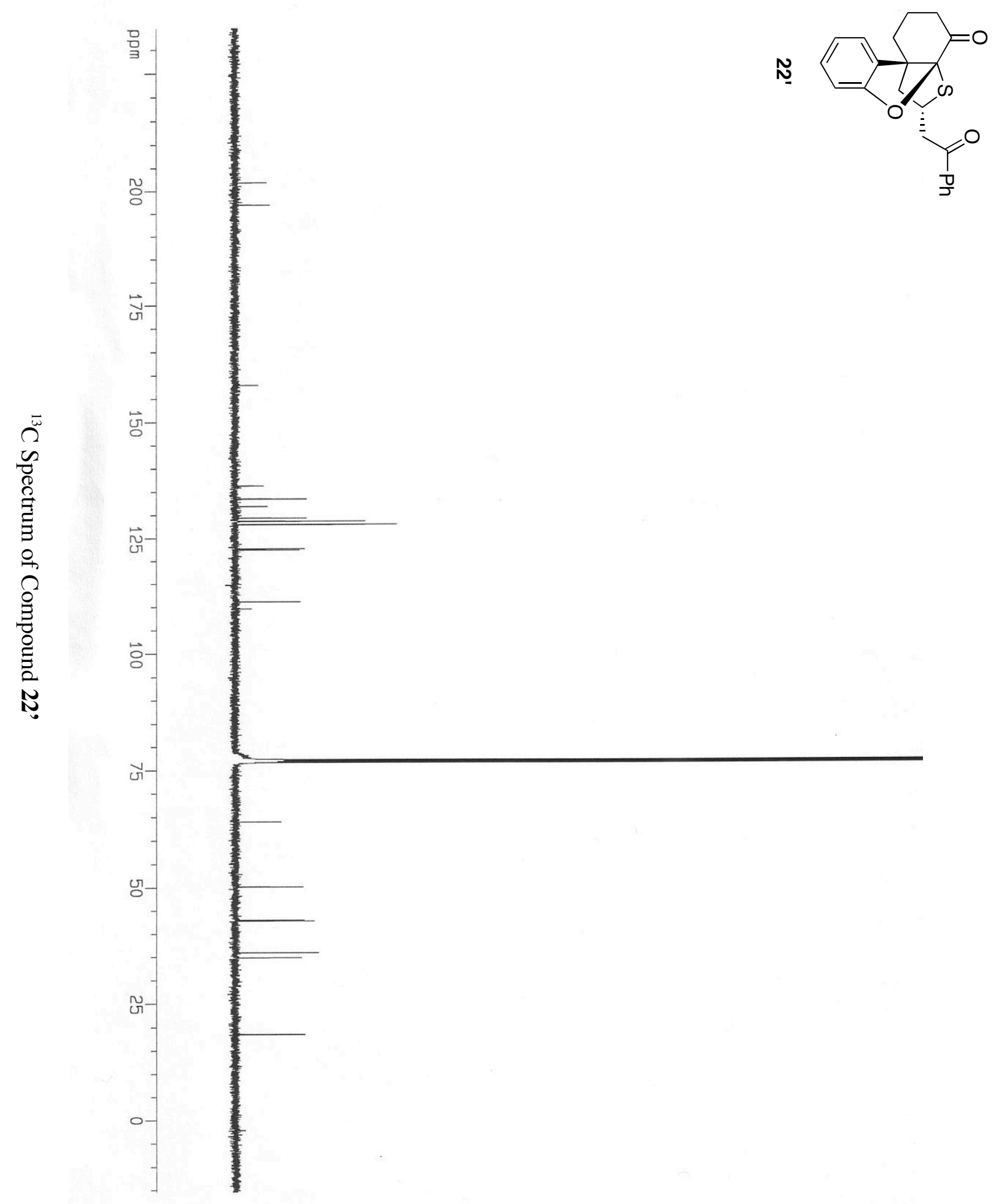

S-111 


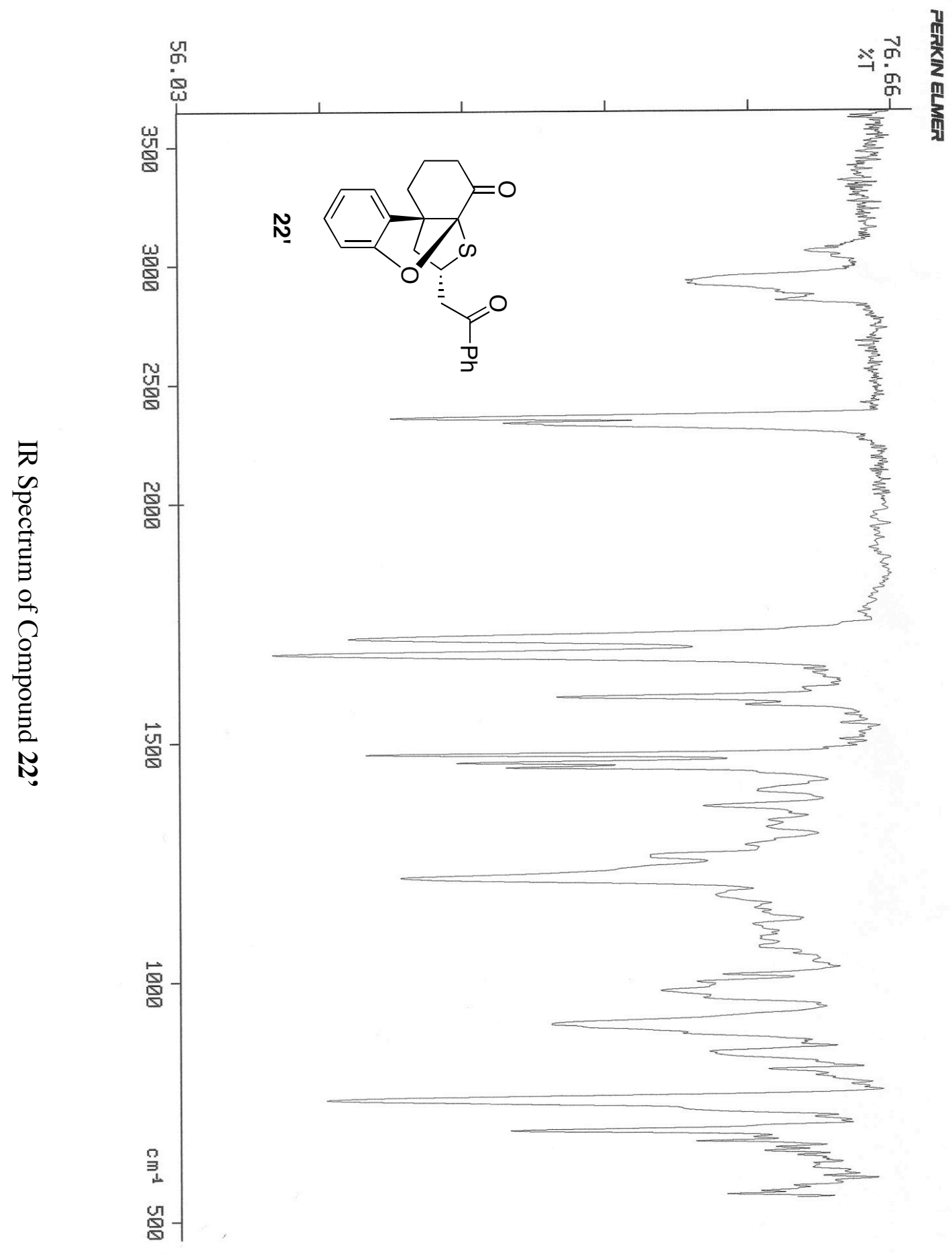

S-112 


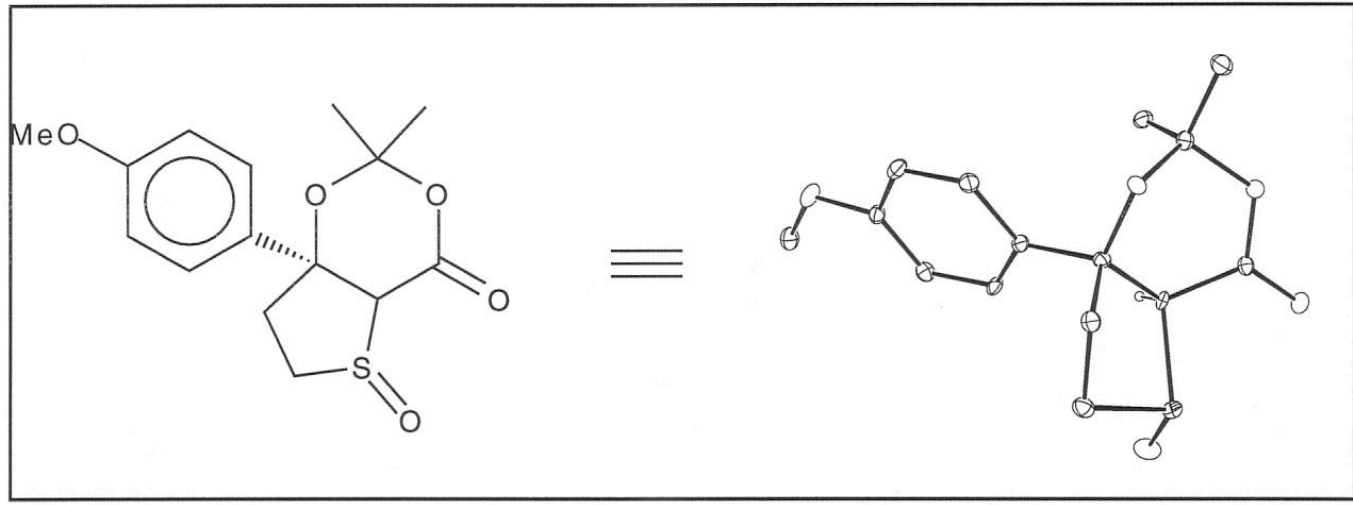

Compound 2025, $\mathrm{C}_{15} \mathrm{H}_{18} \mathrm{SO}_{5}$, crystallizes in the triclinic space group $\mathrm{P} \overline{1}$ with $\mathrm{a}=7.6885(6) \AA$, $b=8.6248(7) \AA, c=12.2086(10) \AA, \alpha=106.449(3)^{\circ}, \beta=89.908(3)^{\circ}, \gamma=111.740(3)^{\circ}, V=716.35(10) \AA^{3}, Z=2$ and $\mathrm{d}_{\text {calc }}=1.439 \mathrm{~g} / \mathrm{cm}^{3}$. X-ray intensity data were collected on a Rigaku Mercury CCD area detector employing graphite-monochromated $\mathrm{Mo}-\mathrm{K}_{\alpha}$ radiation $(\lambda=0.71069 \AA)$ at a temperature of $143^{\circ} \mathrm{K}$. Preliminary indexing was performed from a series of twelve $0.5^{\circ}$ rotation images with exposures of 30 seconds. A total of 802 rotation images were collected with a crystal to detector distance of $36 \mathrm{~mm}$, a $2 \theta$ swing angle of $-10^{\circ}$, rotation widths of $0.5^{\circ}$ and exposures of 30 seconds: scan no. 1 was a $\phi$-scan from $45^{\circ}$ to $105^{\circ}$ at $\omega=10^{\circ}$ and $\chi=20^{\circ}$; scan no. 2 was a $\phi$-scan from $318.75^{\circ}$ to $573.75^{\circ}$ at $\omega=0^{\circ}$ and $\chi$ $=-30^{\circ}$; scan no. 3 was an $\omega$-scan from $-20^{\circ}$ to $4^{\circ}$ at $\chi=-90^{\circ}$ and $\phi=315^{\circ}$; scan no. 4 was an $\omega$-scan from $-20^{\circ}$ to $2^{\circ}$ at $\chi=-90^{\circ}$ and $\phi=135^{\circ}$, and scan no. 5 was an $\omega$-scan from $-20^{\circ}$ to $20^{\circ}$ at $\chi=-90^{\circ}$ and $\phi=225^{\circ}$. Rotation images were processed using CrystalClear', producing a listing of unaveraged $\mathrm{F}^{2}$ and $\sigma\left(\mathrm{F}^{2}\right)$ values which were then passed to the CrystalStructure ${ }^{2}$ program package for further processing and structure solution on a Dell Pentium III computer. A total of 10214 reflections were measured over the ranges $5.34 \leq 2 \theta \leq 50.1^{\circ},-9 \leq h \leq 9,-10 \leq k \leq 10,-14 \leq I \leq 12$ yielding 10214 unique reflections $\left(R_{\text {int }}\right.$ $=0.0000)$. The intensity data were corrected for Lorentz and polarization effects and for absorption. using REQAB $^{3}$ (minimum and maximum transmission 0.500, 1.000).

The structure was solved by direct methods $\left(\operatorname{SIR} 97^{4}\right)$. During data collection, it was noticed that many reflections appeared as double spots. The crystal was found to be twinned by a $180^{\circ}$ about the

\section{S-113}


normal to 001 . Twin indexing and processing of twinned data was performed by the TwinSolve module $^{5}$ of CrystalClear. Refinement was by full-matrix least squares based on $\mathrm{F}^{2}$ using SHELXL-97 ${ }^{6}$. All reflections were used during refinement $\left(\mathrm{F}^{2}\right.$ 's that were experimentally negative were replaced by $\mathrm{F}^{2}=$ $0)$. The weighting scheme used was $w=1 /\left[\sigma^{2}\left(F_{0}^{2}\right)+0.1456 P^{2}+1.5919 P\right]$ where $P=\left(F_{0}^{2}+2 F_{c}^{2}\right) / 3$. Nonhydrogen atoms were refined anisotropically and hydrogen atoms were refined using a "riding" model. Refinement converged to $R_{1}=0.0806$ and $w R_{2}=0.2385$ for 9804 reflections for which $F>4 \sigma(F)$ and $R_{1}=0.0823, w R_{2}=0.2414$ and $G O F=1.128$ for all 10214 unique, non-zero reflections and 192 variables $^{7}$. The maximum $\Delta / \sigma$ in the final cycle of least squares was 0.000 and the two most prominent peaks in the final difference Fourier were +0.486 and $-1.002 \mathrm{e} / \AA^{3}$.

Table 1. lists cell information, data collection parameters, and refinement data. Final positional and equivalent isotropic thermal parameters are given in Table 2. Anisotropic thermal parameters are in Table 3. Tables 4. and 5. list bond distances and bond angles. Figure 1. is an ORTEP ${ }^{8}$ representation of the molecule with $30 \%$ probability thermal ellipsoids displayed.

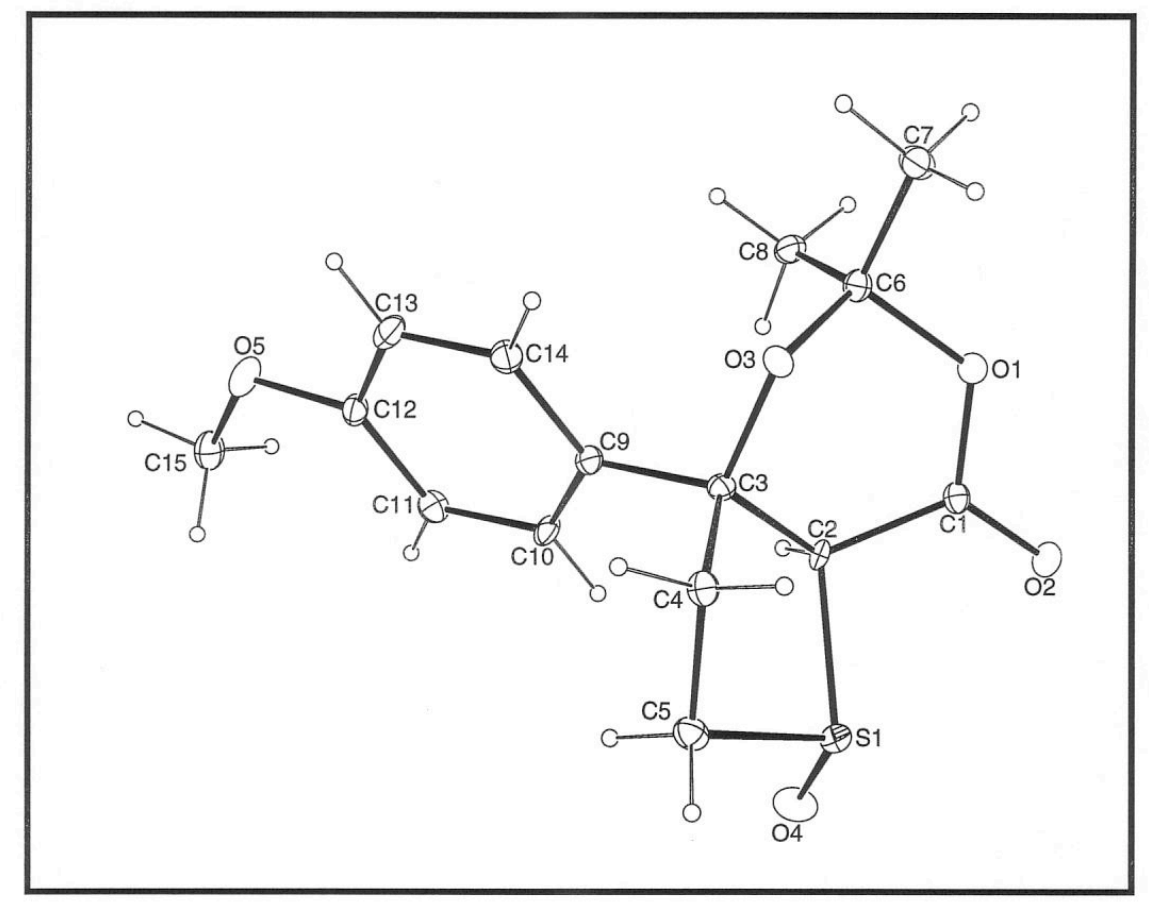

Figure 1. ORTEP drawing of the title compound with $30 \%$ probability thermal ellipsoids. 


\section{References}

1. CrystalClear: Rigaku Corporation, 1999.

2. CrystalStructure: Crystal Structure Analysis Package, Rigaku Corp. Rigaku/MSC (2002).

3. REQAB4: R.A. Jacobsen, (1994). Private Communication.

4. SIR97: Altomare, A., M. Burla, M. Camalli, G. Cascarano, C. Giacovazzo, A. Guagliardi, A. Moliterni, G. Polidori \& R. Spagna (1999). J. Appl. Cryst., 32, 115-119.

5. TwinSolve: Christer Swensson, MaxLab, Lund, Sweden, Private Communication.

6. SHELXL-97: Program for the Refinement of Crystal Structures, Sheldrick, G.M. (1997), University of Göttingen, Germany.

7. $R_{1}=\sum\left\|F_{0}|-| F_{C}\right\| / \sum\left|F_{0}\right|$

$w R_{2}=\left\{\sum w\left(F_{0}^{2}-F_{c}^{2}\right)^{2} / \sum w\left(F_{o}^{2}\right)^{2}\right\}^{1 / 2}$

$\mathrm{GOF}=\left\{\sum w\left(\mathrm{~F}_{0}^{2}-\mathrm{F}_{\mathrm{c}}^{2}\right)^{2} /(n-p)\right\}^{1 / 2}$

where $n=$ the number of reflections and $p=$ the number of parameters refined.

8. "ORTEP-II: A Fortran Thermal Ellipsoid Plot Program for Crystal Structure Illustrations". C.K. Johnson (1976) ORNL-5138. 
Table 1. Summary of Structure Determination of Compound 2025

\begin{tabular}{|c|c|}
\hline Formula: & $\mathrm{C}_{15} \mathrm{H}_{18} \mathrm{SO}_{5}$ \\
\hline Formula weight: & 310.35 \\
\hline Crystal class: & triclinic \\
\hline Space group: & $\mathrm{P} \overline{1}(\# 2)$ \\
\hline Z & 2 \\
\hline \multicolumn{2}{|l|}{ Cell constants: } \\
\hline a & $7.6885(6) \AA$ \\
\hline$b$ & $8.6248(7) \AA$ \\
\hline $\mathrm{C}$ & $12.2086(10) \AA$ \\
\hline$\alpha$ & $106.449(3)^{\circ}$ \\
\hline$\beta$ & $89.908(3)^{\circ}$ \\
\hline$\gamma$ & $111.740(3)^{\circ}$ \\
\hline V & $716.35(10) \AA^{3}$ \\
\hline$\mu$ & $2.45 \mathrm{~cm}^{-1}$ \\
\hline crystal size, mm & $0.42 \times 0.20 \times 0.18$ \\
\hline$D_{\text {calc }}$ & $1.439 \mathrm{~g} / \mathrm{cm}^{3}$ \\
\hline$F(000)$ & 328 \\
\hline Radiation: & $\operatorname{Mo}-K_{\alpha}(\lambda=0.71069 \AA)$ \\
\hline $2 \theta$ range & $5.34-50.1^{\circ}$ \\
\hline hkl collected: & $-9 \leq \mathrm{h} \leq 9 ;-10 \leq \mathrm{k} \leq 10 ;-14 \leq \mathrm{I} \leq 12$ \\
\hline No. reflections measured: & 10214 \\
\hline No. unique reflections: & $10214\left(\mathrm{R}_{\mathrm{int}}=0.0000\right)$ \\
\hline No. observed reflections & $9804(F>4 \sigma)$ \\
\hline No. reflections used in refinement & 10214 \\
\hline No. parameters & 192 \\
\hline \multirow[t]{2}{*}{$R$ indices $(F>4 \sigma)$} & $R_{1}=0.0806$ \\
\hline & $w R_{2}=0.2385$ \\
\hline \multirow[t]{2}{*}{$\mathrm{R}$ indices (all data) } & $\mathrm{R}_{1}=0.0823$ \\
\hline & $w R_{2}=0.2414$ \\
\hline GOF: & 1.128 \\
\hline Final Difference Peaks, e/ $\AA^{3}$ & $+0.486,-1.002$ \\
\hline
\end{tabular}


Table 2. Refined Positional Parameters for Compound 2025

\begin{tabular}{|c|c|c|c|c|}
\hline Atom & $\mathrm{x}$ & $y$ & $z$ & $\cup_{e d}, \AA^{2}$ \\
\hline $\mathrm{C} 1$ & $0.3922(3)$ & $0.1378(3)$ & $0.9921(2)$ & $0.0147(5)$ \\
\hline $\mathrm{C} 2$ & $0.4782(3)$ & $0.2409(3)$ & $0.9093(2)$ & $0.0136(5)$ \\
\hline $\mathrm{H} 2$ & 0.4291 & 0.3335 & 0.9204 & 0.018 \\
\hline C3 & $0.4370(3)$ & $0.1366(3)$ & $0.7801(2)$ & $0.0137(5)$ \\
\hline C4 & $0.6234(3)$ & $0.1180(3)$ & $0.7459(2)$ & $0.0165(5)$ \\
\hline $\mathrm{H} 4 \mathrm{a}$ & 0.6423 & 0.0298 & 0.7735 & 0.022 \\
\hline $\mathrm{H} 4 \mathrm{~b}$ & 0.6225 & 0.0849 & 0.6630 & 0.022 \\
\hline C5 & $0.7782(3)$ & $0.2979(3)$ & $0.8024(2)$ & $0.0195(5)$ \\
\hline $\mathrm{H} 5 \mathrm{a}$ & 0.7682 & 0.3841 & 0.7688 & 0.026 \\
\hline $\mathrm{H} 5 \mathrm{~b}$ & 0.9020 & 0.2937 & 0.7943 & 0.026 \\
\hline C6 & $0.1549(3)$ & $-0.0606(3)$ & $0.8313(2)$ & $0.0176(5)$ \\
\hline $\mathrm{C7}$ & $0.0414(4)$ & $-0.2555(3)$ & $0.8020(2)$ & $0.0230(6)$ \\
\hline $\mathrm{H} 7 \mathrm{a}$ & -0.0607 & -0.2759 & 0.8484 & 0.035 \\
\hline $\mathrm{H} 7 \mathrm{~b}$ & 0.1211 & -0.3124 & 0.8168 & 0.035 \\
\hline $\mathrm{H} 7 \mathrm{c}$ & -0.0081 & -0.3018 & 0.7222 & 0.035 \\
\hline C8 & $0.0353(3)$ & $0.0421(3)$ & $0.8258(2)$ & $0.0207(5)$ \\
\hline $\mathrm{H} 8 \mathrm{a}$ & -0.0611 & 0.0192 & 0.8759 & 0.031 \\
\hline $\mathrm{H} 8 \mathrm{~b}$ & -0.0222 & 0.0075 & 0.7484 & 0.031 \\
\hline $\mathrm{H} 8 \mathrm{c}$ & 0.1129 & 0.1650 & 0.8495 & 0.031 \\
\hline C9 & $0.3756(3)$ & $0.2266(3)$ & $0.7049(2)$ & $0.0152(5)$ \\
\hline C10 & $0.4225(3)$ & $0.4060(3)$ & $0.7355(2)$ & $0.0170(5)$ \\
\hline $\mathrm{H} 10$ & 0.4910 & 0.4762 & 0.8066 & 0.023 \\
\hline C11 & $0.3701(3)$ & $0.4844(3)$ & $0.6629(2)$ & $0.0189(5)$ \\
\hline $\mathrm{H} 11$ & 0.4046 & 0.6052 & 0.6850 & 0.025 \\
\hline C12 & $0.2659(3)$ & $0.3801(3)$ & $0.5576(2)$ & $0.0175(5)$ \\
\hline $\mathrm{C} 13$ & $0.2182(3)$ & $0.2000(3)$ & $0.5254(2)$ & $0.0220(5)$ \\
\hline $\mathrm{H} 13$ & 0.1488 & 0.1298 & 0.4546 & 0.029 \\
\hline C14 & $0.2726(3)$ & $0.1245(3)$ & $0.5976(2)$ & $0.0196(5)$ \\
\hline $\mathrm{H} 14$ & 0.2404 & 0.0040 & 0.5744 & 0.026 \\
\hline C15 & $0.2547(4)$ & $0.6243(3)$ & $0.5055(2)$ & $0.0247(6)$ \\
\hline $\mathrm{H} 15 \mathrm{a}$ & 0.2019 & 0.6485 & 0.4442 & 0.037 \\
\hline $\mathrm{H} 15 \mathrm{~b}$ & 0.3896 & 0.6819 & 0.5145 & 0.037 \\
\hline $\mathrm{H} 15 \mathrm{c}$ & 0.2071 & 0.6666 & 0.5757 & 0.037 \\
\hline $\mathrm{S} 1$ & $0.73620(8)$ & $0.34895(7)$ & $0.95241(5)$ & $0.0179(2)$ \\
\hline O1 & $0.2360(2)$ & $-0.0067(2)$ & $0.95188(12)$ & $0.0187(4)$ \\
\hline $\mathrm{O} 2$ & $0.4557(2)$ & $0.1843(2)$ & $1.09067(13)$ & $0.0236(4)$ \\
\hline
\end{tabular}




\begin{tabular}{|lllll|}
\hline O3 & $0.3025(2)$ & $-0.0392(2)$ & $0.76163(12)$ & $0.0151(4)$ \\
O4 & $0.7826(2)$ & $0.5423(2)$ & $0.99874(14)$ & $0.0287(5)$ \\
O5 & $0.2035(2)$ & $0.4400(2)$ & $0.47906(14)$ & $0.0265(4)$ \\
\hline & $\mathrm{U}_{\mathrm{eq}=}{ }^{1} / 3\left[\mathrm{U}_{11}\left(\mathrm{aa}^{*}\right)^{2}+\mathrm{U}_{22}\left(\mathrm{bb}^{*}\right)^{2}+\mathrm{U}_{33}\left(\mathrm{cc}^{*}\right)^{2}+2 \mathrm{U}_{12} \mathrm{aa}^{*} \mathrm{bb}^{*} \cos \gamma+2 \mathrm{U}_{13} \mathrm{aa}^{*} \mathrm{cc}{ }^{*} \cos \beta+2 \mathrm{U}_{23} \mathrm{bb}^{*} \mathrm{cc}{ }^{*} \cos \alpha\right]$ \\
\hline
\end{tabular}

S-118 
Table 3. Refined Thermal Parameters (U's) for Compound 2025

\begin{tabular}{|c|c|c|c|c|c|c|}
\hline Atom & $\bigcup_{11}$ & $\mathrm{U}_{22}$ & $\mathrm{U}_{33}$ & $\mathrm{U}_{23}$ & $U_{13}$ & $U_{12}$ \\
\hline $\mathrm{C} 1$ & $0.0184(12)$ & $0.0144(11)$ & $0.0124(12)$ & $0.0042(9)$ & $0.0034(9)$ & $0.0076(10)$ \\
\hline $\mathrm{C} 2$ & $0.0168(11)$ & $0.0173(11)$ & $0.0090(11)$ & $0.0057(9)$ & $-0.0009(8)$ & $0.0079(10)$ \\
\hline C3 & $0.0140(11)$ & $0.0122(11)$ & $0.0124(11)$ & $0.0021(9)$ & $0.0008(9)$ & $0.0033(9)$ \\
\hline $\mathrm{C} 4$ & $0.0185(12)$ & $0.0177(11)$ & $0.0142(12)$ & $0.0040(9)$ & $0.0044(9)$ & $0.0085(10)$ \\
\hline C5 & $0.0153(12)$ & $0.0210(12)$ & $0.0234(13)$ & $0.0083(10)$ & $0.0047(9)$ & $0.0071(10)$ \\
\hline C6 & $0.0191(12)$ & $0.0202(12)$ & $0.0115(12)$ & $0.0047(9)$ & $0.0027(9)$ & $0.0054(10)$ \\
\hline $\mathrm{C} 7$ & $0.0240(13)$ & $0.0194(12)$ & $0.0191(13)$ & $0.0055(10)$ & $0.0029(10)$ & $0.0014(11)$ \\
\hline C8 & $0.0139(12)$ & $0.0279(13)$ & $0.0169(12)$ & $0.0071(10)$ & $0.0002(9)$ & $0.0042(11)$ \\
\hline $\mathrm{Cg}$ & $0.0137(11)$ & $0.0204(12)$ & $0.0098(11)$ & $0.0032(9)$ & $0.0033(9)$ & $0.0057(10)$ \\
\hline $\mathrm{C} 10$ & $0.0196(12)$ & $0.0199(12)$ & $0.0085(11)$ & $0.0015(9)$ & $-0.0020(9)$ & $0.0065(10)$ \\
\hline $\mathrm{C} 11$ & $0.0213(12)$ & $0.0162(11)$ & $0.0202(13)$ & $0.0061(10)$ & $0.0043(9)$ & $0.0079(10)$ \\
\hline $\mathrm{C} 12$ & $0.0166(12)$ & $0.0240(12)$ & $0.0153(12)$ & $0.0097(10)$ & $0.0020(9)$ & $0.0087(10)$ \\
\hline C13 & $0.0237(13)$ & $0.0232(12)$ & $0.0147(12)$ & $0.0034(10)$ & $-0.0046(9)$ & $0.0060(11)$ \\
\hline C14 & $0.0231(13)$ & $0.0143(11)$ & $0.0162(12)$ & $0.0024(9)$ & $0.0008(10)$ & $0.0032(10)$ \\
\hline C15 & $0.0299(14)$ & $0.0273(13)$ & $0.0238(13)$ & $0.0130(11)$ & $0.0038(10)$ & $0.0148(12)$ \\
\hline S1 & $0.0155(3)$ & $0.0183(3)$ & $0.0169(3)$ & $0.0049(2)$ & $-0.0020(2)$ & $0.0035(3)$ \\
\hline 01 & $0.0189(9)$ & $0.0198(8)$ & $0.0128(8)$ & $0.0048(7)$ & $0.0010(7)$ & $0.0025(7)$ \\
\hline $\mathrm{O} 2$ & $0.0263(10)$ & $0.0279(10)$ & $0.0121(9)$ & $0.0069(7)$ & $-0.0013(7)$ & $0.0048(8)$ \\
\hline O3 & $0.0156(8)$ & $0.0141(8)$ & $0.0139(8)$ & $0.0040(6)$ & $0.0046(6)$ & $0.0042(7)$ \\
\hline $\mathrm{O} 4$ & $0.0247(10)$ & $0.0214(10)$ & $0.0289(10)$ & $-0.0003(8)$ & $0.0029(8)$ & $0.0025(8)$ \\
\hline O5 & $0.0344(11)$ & $0.0269(9)$ & $0.0196(9)$ & $0.0079(7)$ & $-0.0067(7)$ & $0.0129(9)$ \\
\hline
\end{tabular}


Table 4. Bond Distances in Compound 2025, $\AA$

\begin{tabular}{|llllll|}
\hline C1-O2 & $1.196(3)$ & $\mathrm{C} 1-\mathrm{O} 1$ & $1.336(3)$ & $\mathrm{C} 1-\mathrm{C} 2$ & $1.524(3)$ \\
$\mathrm{C} 2-\mathrm{C} 3$ & $1.544(3)$ & $\mathrm{C} 2-\mathrm{S} 1$ & $1.854(2)$ & $\mathrm{C} 3-\mathrm{O} 3$ & $1.437(3)$ \\
$\mathrm{C} 3-\mathrm{C} 9$ & $1.532(3)$ & $\mathrm{C} 3-\mathrm{C} 4$ & $1.544(3)$ & $\mathrm{C} 4-\mathrm{C} 5$ & $1.525(3)$ \\
$\mathrm{C} 5-\mathrm{S} 1$ & $1.816(2)$ & $\mathrm{C} 6-\mathrm{O} 3$ & $1.405(3)$ & $\mathrm{C} 6-\mathrm{O} 1$ & $1.474(3)$ \\
$\mathrm{C} 6-\mathrm{C} 8$ & $1.508(3)$ & $\mathrm{C} 6-\mathrm{C} 7$ & $1.512(3)$ & $\mathrm{C} 9-\mathrm{C} 10$ & $1.386(3)$ \\
$\mathrm{C} 9-\mathrm{C} 14$ & $1.397(3)$ & $\mathrm{C} 10-\mathrm{C} 11$ & $1.395(3)$ & $\mathrm{C} 11-\mathrm{C} 12$ & $1.385(3)$ \\
$\mathrm{C} 12-\mathrm{O} 5$ & $1.372(3)$ & $\mathrm{C} 12-\mathrm{C} 13$ & $1.391(3)$ & $\mathrm{C} 13-\mathrm{C} 14$ & $1.379(3)$ \\
$\mathrm{C} 15-\mathrm{O} 5$ & $1.425(3)$ & $\mathrm{S} 1-\mathrm{O} 4$ & $1.501(2)$ & & \\
\hline
\end{tabular}

Table 5. Bond Angles in Compound 2025, ${ }^{\circ}$

\begin{tabular}{|llllll|}
\hline O2-C1-O1 & $119.9(2)$ & O2-C1-C2 & $122.7(2)$ & O1-C1-C2 & $117.4(2)$ \\
C1-C2-C3 & $116.4(2)$ & C1-C2-S1 & $107.68(14)$ & C3-C2-S1 & $110.1(2)$ \\
O3-C3-C9 & $111.1(2)$ & O3-C3-C2 & $111.0(2)$ & C9-C3-C2 & $113.6(2)$ \\
O3-C3-C4 & $105.4(2)$ & C9-C3-C4 & $110.0(2)$ & C2-C3-C4 & $105.2(2)$ \\
C5-C4-C3 & $105.6(2)$ & C4-C5-S1 & $103.9(2)$ & O3-C6-O1 & $108.6(2)$ \\
O3-C6-C8 & $115.6(2)$ & O1-C6-C8 & $107.5(2)$ & O3-C6-C7 & $106.8(2)$ \\
O1-C6-C7 & $104.8(2)$ & C8-C6-C7 & $113.0(2)$ & C10-C9-C14 & $117.7(2)$ \\
C10-C9-C3 & $123.6(2)$ & C14-C9-C3 & $118.7(2)$ & C9-C10-C11 & $122.0(2)$ \\
C12-C11-C10 & $119.2(2)$ & O5-C12-C11 & $125.1(2)$ & O5-C12-C13 & $115.4(2)$ \\
C11-C12-C13 & $119.5(2)$ & C14-C13-C12 & $120.6(2)$ & C13-C14-C9 & $120.9(2)$ \\
O4-S1-C5 & $108.43(10)$ & O4-S1-C2 & $107.03(10)$ & C5-S1-C2 & $90.36(10)$ \\
C1-O1-C6 & $119.7(2)$ & C6-O3-C3 & $116.6(2)$ & C12-O5-C15 & $117.8(2)$ \\
\hline
\end{tabular}


X-ray Structure Determination of Compound 2026

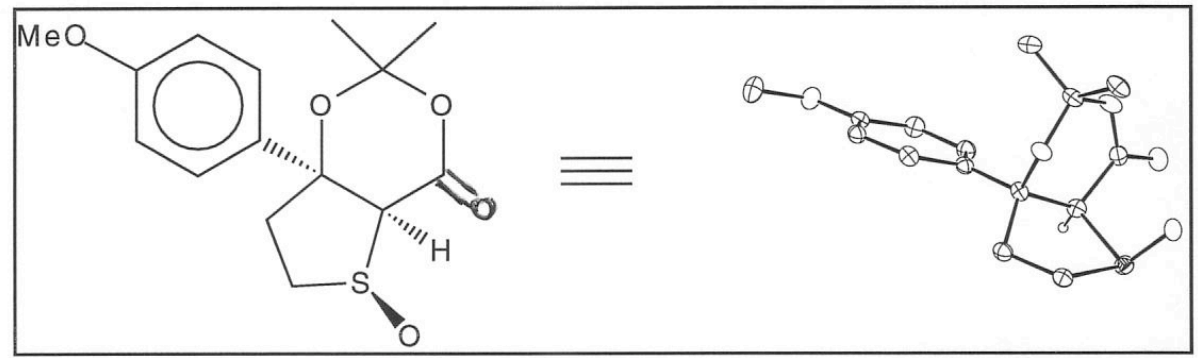

Compound 2026, $\mathrm{C}_{15} \mathrm{H}_{18} \mathrm{SO}_{5}$, crystallizes in the orthorhombic space group $\mathrm{Pna2}_{1}$ (systematic

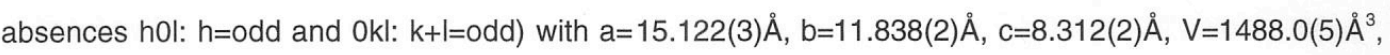
$\mathrm{Z}=4$ and $\mathrm{d}_{\mathrm{calc}}=1.385 \mathrm{~g} / \mathrm{cm}^{3}$. X-ray intensity data were collected on a Rigaku Mercury CCD aiea detector employing graphite-monochromated Mo- $\mathrm{K}_{\alpha}$ radiation $(\lambda=0.71069 \AA)$ at a temperature of $143^{\circ} \mathrm{K}$. Preliminary indexing was performed from a series of twelve $0.5^{\circ}$ rotation images with exposures of 30 seconds. A total of 218 rotation images were collected with a crystal to detector distance of $36 \mathrm{~mm}$, a $2 \theta$ swing angle of $-10^{\circ}$, rotation widths of $0.5^{\circ}$ and exposures of 120 seconds: scan no. 1 was a $\phi$-scan from $155^{\circ}$ to $264^{\circ}$ at $\omega=0^{\circ}$ and $\chi=0^{\circ}$. Rotation images were processed using CrystalClear', producing a listing of unaveraged $\mathrm{F}^{2}$ and $\sigma\left(\mathrm{F}^{2}\right)$ values which were then passed to the CrystalStructure program package for further processing and structure solution on a Dell Pentium III computer. A total of 4175 reflections were measured over the ranges $5.38 \leq 2 \theta \leq 50.68^{\circ}, \quad-18 \leq h \leq 14, \quad-11 \leq k \leq 14, \quad-6 \leq 1 \leq 9$ yielding 2302 unique reflections $\left(R_{\text {int }}=0.0310\right)$. The intensity data were corrected for Lorentz and polarization effects and for absorption. using $\mathrm{REQAB}^{3}$ (minimum and maximum transmission 0.753 , 1.000).

The structure was solved by direct methods $\left(\operatorname{SIR} 97^{4}\right)$. Refinement was by full-matrix least squares based on $\mathrm{F}^{2}$ using SHELXL-97 ${ }^{5}$. All reflections were used during refinement $\left(\mathrm{F}^{2}\right.$ 's that were experimentally negative were replaced by $\left.F^{2}=0\right)$. The weighting scheme used was $w=1 /\left[\sigma^{2}\left(F_{0}^{2}\right)+\right.$ $\left.0.0446 \mathrm{P}^{2}+0.5525 \mathrm{P}\right]$ where $\mathrm{P}=\left(\mathrm{F}_{0}^{2}+2 \mathrm{~F}_{\mathrm{c}}^{2}\right) / 3$. Non-hydrogen atoms were refined anisotropically and hydrogen atoms were refined using a "riding" model. Refinement converged to $R_{1}=0.0445$ and $w R_{2}=0.0982$ for 1992 reflections for which $F>4 \sigma(F)$ and $R_{1}=0.0546, w R_{2}=0.1064$ and $G O F=1.075$ for all 
2302 unique, non-zero reflections and 194 variables $^{6}$. The maximum $\Delta / \sigma$ in the final cycle of least squares was 0.000 and the two most prominent peaks in the final difference Fourier were +0.284 and $0.243 \mathrm{e} / \AA^{3}$.

Table 1. lists cell information, data collection parameters, and refinement data. Final positional and equivalent isotropic thermal parameters are given in Table 2. Anisotropic thermal parameters are in Table 3. Tables 4. and 5. list bond distances and bond angles. Figure 1. is an ORTEP ${ }^{7}$ representation of the molecule with $30 \%$ probability thermal ellipsoids displayed.

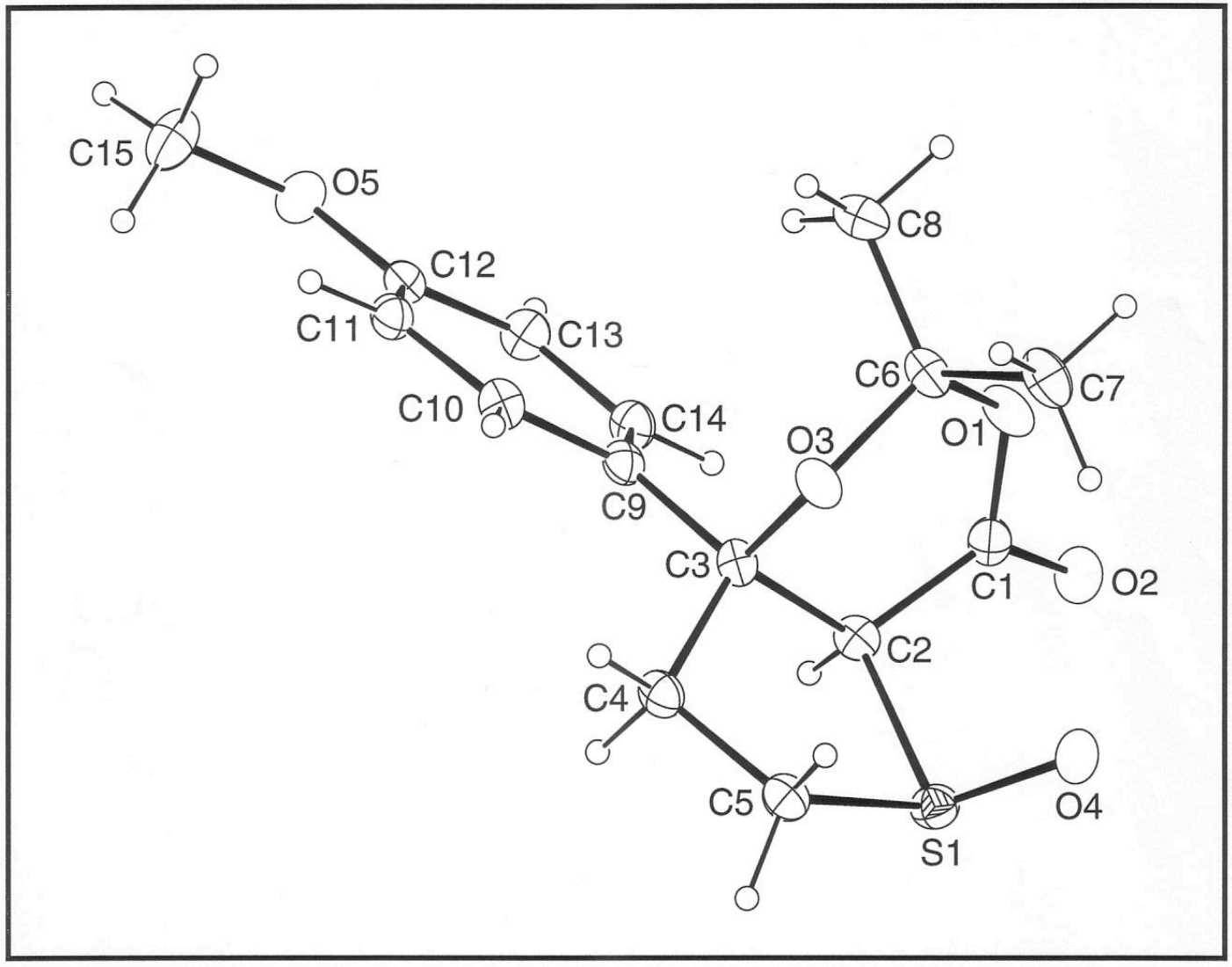

Figure 1. ORTEP drawing of the title compound with $30 \%$ probability thermal ellipsoids. 


\section{References}

1. CrystalClear: Rigaku Corporation, 1999.

2. CrystalStructure: Crystal Structure Analysis Package, Rigaku Corp. Rigaku/MSC (2002).

3. REQAB4: R.A. Jacobsen, (1994). Private Communication.

4. SIR97: Altomare, A., M. Burla, M. Camalli, G. Cascarano, C. Giacovazzo, A. Guagliardi, A. Moliterni, G. Polidori \& R. Spagna (1999). J. Appl. Cryst., 32, 115-119.

5. SHELXL-97: Program for the Refinement of Crystal Structures, Sheldrick, G.M. (1997), University of Göttingen, Germany.

6. $\mathrm{R}_{1}=\sum\left\|\mathrm{F}_{\mathrm{o}}|-| \mathrm{F}_{\mathrm{C}}\right\| / \sum\left|\mathrm{F}_{\mathrm{o}}\right|$

$w R_{2}=\left\{\sum w\left(F_{0}^{2}-F_{c}^{2}\right)^{2} / \sum w\left(F_{0}^{2}\right)^{2}\right\}^{1 / 2}$

GOF $=\left\{\sum w\left(F_{0}^{2}-F_{c}^{2}\right)^{2} /(n-p)\right\}^{1 / 2}$

where $n=$ the number of reflections and $p=$ the number of parameters refined.

7. "ORTEP-II: A Fortran Thermal Ellipsoid Plot Program for Crystal Structure Illustrations". C.K. Johnson (1976) ORNL-5138. 
Table 1. Summary of Structure Determination of Compound 2026

Formula:

Formula weight:

Crystal class:

Space group:

Z

Cell constants:

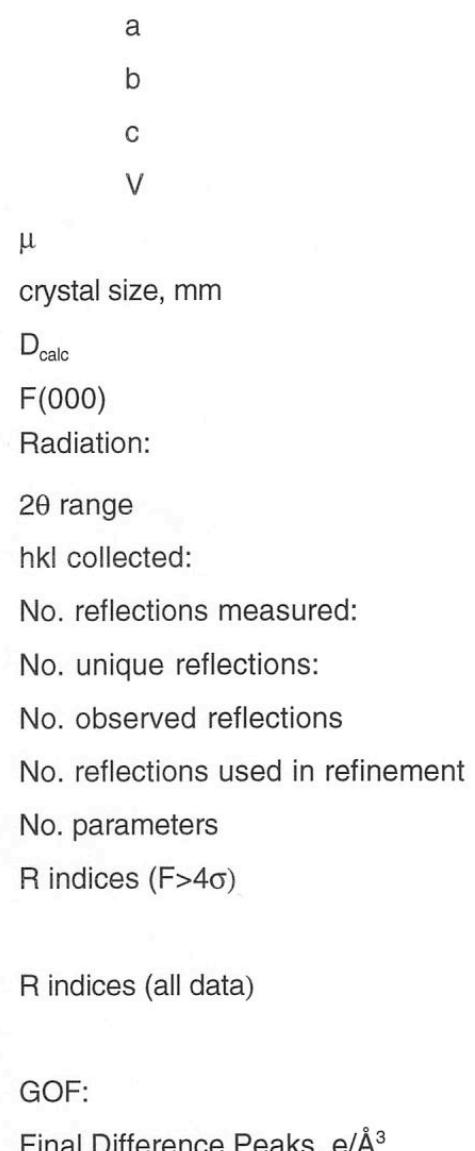

$\mathrm{C}_{15} \mathrm{H}_{18} \mathrm{SO}_{5}$

310.35

orthorhombic

Pna2 $_{1}$ (\#33)

4

$15.122(3) \AA$
$11.838(2) \AA$
$8.312(2) \AA$
$1488.0(5) \AA^{3}$
$2.36 \mathrm{~cm}^{-1}$
$0.42 \times 0.03 \times 0.02$
$1.385 \mathrm{~g} / \mathrm{cm}^{3}$
656
$M o-K_{\alpha}(\lambda=0.71069 \AA)$
$5.38-50.68 \circ$
$-18 \leq h \leq 14 ;-11 \leq \mathrm{k} \leq 14 ; \quad-6 \leq \mathrm{I} \leq 9$
4175
$2302\left(\mathrm{R}_{\mathrm{int}}=0.0310\right)$
$1992(\mathrm{~F}>4 \sigma)$
2302
194
$\mathrm{R}_{1}=0.0445$
$\mathrm{wR}_{2}=0.0982$
$\mathrm{R}_{1}=0.0546$
$\mathrm{wR}_{2}=0.1064$
1.075
$+0.284,-0.243$


Table 2. Refined Positional Parameters for Compound 2026

\begin{tabular}{|c|c|c|c|c|}
\hline Atom & $x$ & y & z & $U_{e d}, \AA^{2}$ \\
\hline $\mathrm{S} 1$ & $0.54005(6)$ & $0.64157(7)$ & $0.09880(12)$ & $0.0303(2)$ \\
\hline $\mathrm{C} 1$ & $0.4161(2)$ & $0.4851(3)$ & $0.0284(5)$ & $0.0280(8)$ \\
\hline $\mathrm{C} 2$ & $0.4369(2)$ & $0.5666(3)$ & $0.1607(5)$ & $0.0261(8)$ \\
\hline $\mathrm{H} 2$ & 0.4485 & 0.5242 & 0.2597 & 0.035 \\
\hline C3 & $0.3650(3)$ & $0.6546(3)$ & $0.1938(5)$ & $0.0267(9)$ \\
\hline $\mathrm{C} 4$ & $0.4151(2)$ & $0.7551(3)$ & $0.2668(5)$ & $0.0289(9)$ \\
\hline $\mathrm{H} 4 \mathrm{a}$ & 0.3774 & 0.8213 & 0.2699 & 0.038 \\
\hline $\mathrm{H} 4 \mathrm{~b}$ & 0.4337 & 0.7376 & 0.3757 & 0.038 \\
\hline C5 & $0.4959(2)$ & $0.7774(3)$ & $0.1604(5)$ & $0.0330(10)$ \\
\hline $\mathrm{H} 5 \mathrm{a}$ & 0.5402 & 0.8195 & 0.2199 & 0.044 \\
\hline $\mathrm{H} 5 \mathrm{~b}$ & 0.4789 & 0.8212 & 0.0668 & 0.044 \\
\hline C6 & $0.3044(3)$ & $0.6192(3)$ & $-0.0767(5)$ & $0.0297(9)$ \\
\hline $\mathrm{C} 7$ & $0.3294(3)$ & $0.6672(4)$ & $-0.2392(5)$ & $0.0432(11)$ \\
\hline $\mathrm{H} 7 \mathrm{a}$ & 0.3023 & 0.7399 & -0.2525 & 0.065 \\
\hline $\mathrm{H} 7 \mathrm{~b}$ & 0.3093 & 0.6172 & -0.3225 & 0.065 \\
\hline $\mathrm{H} 7 \mathrm{c}$ & 0.3925 & 0.6748 & -0.2456 & 0.065 \\
\hline $\mathrm{C} 8$ & $0.2068(3)$ & $0.5944(4)$ & $-0.0586(5)$ & $0.0435(11)$ \\
\hline $\mathrm{H} 8 \mathrm{a}$ & 0.1964 & 0.5581 & 0.0430 & 0.065 \\
\hline $\mathrm{H} 8 \mathrm{~b}$ & 0.1879 & 0.5455 & -0.1441 & 0.065 \\
\hline $\mathrm{H} 8 \mathrm{c}$ & 0.1741 & 0.6638 & -0.0632 & 0.065 \\
\hline $\mathrm{C9}$ & $0.2888(2)$ & $0.6180(3)$ & $0.2996(5)$ & $0.0252(8)$ \\
\hline C10 & $0.2267(3)$ & $0.6974(3)$ & $0.3508(4)$ & $0.0291(9)$ \\
\hline $\mathrm{H} 10$ & 0.2323 & 0.7718 & 0.3163 & 0.039 \\
\hline C11 & $0.1571(2)$ & $0.6691(3)$ & $0.4511(5)$ & $0.0289(9)$ \\
\hline $\mathrm{H} 11$ & 0.1174 & 0.7242 & 0.4851 & 0.038 \\
\hline C12 & $0.1472(2)$ & $0.5582(3)$ & $0.5003(5)$ & $0.0275(8)$ \\
\hline C13 & $0.2063(2)$ & $0.4766(3)$ & $0.4505(5)$ & $0.0321(9)$ \\
\hline $\mathrm{H} 13$ & 0.1989 & 0.4018 & 0.4823 & 0.043 \\
\hline C14 & $0.2769(3)$ & $0.5066(3)$ & $0.3528(4)$ & $0.0296(9)$ \\
\hline $\mathrm{H} 14$ & 0.3173 & 0.4515 & 0.3218 & 0.039 \\
\hline C15 & $0.0176(3)$ & $0.6022(4)$ & $0.6573(6)$ & $0.0436(11)$ \\
\hline $\mathrm{H} 15 \mathrm{a}$ & 0.0479 & 0.6652 & 0.7049 & 0.065 \\
\hline $\mathrm{H} 15 \mathrm{~b}$ & -0.0202 & 0.5678 & 0.7360 & 0.065 \\
\hline $\mathrm{H} 15 \mathrm{c}$ & -0.0173 & 0.6280 & 0.5681 & 0.065 \\
\hline 01 & $0.3484(2)$ & $0.5080(2)$ & $-0.0673(3)$ & $0.0379(7)$ \\
\hline $\mathrm{O} 2$ & $0.4583(2)$ & $0.3993(2)$ & $0.0086(4)$ & $0.0356(6)$ \\
\hline O3 & $0.3367(2)$ & $0.6960(2)$ & $0.0380(3)$ & $0.0279(6)$ \\
\hline O4 & $0.5427(2)$ & $0.6437(2)$ & $-0.0813(3)$ & $0.0338(7)$ \\
\hline $\mathrm{O5}$ & $0.0811(2)$ & $0.5210(2)$ & $0.6020(3)$ & $0.0349(6)$ \\
\hline
\end{tabular}


Table 3. Refined Thermal Parameters (U's) for Compound 2026

\begin{tabular}{|c|c|c|c|c|c|c|}
\hline Atom & $\mathrm{U}_{11}$ & $\mathrm{U}_{22}$ & $\mathrm{U}_{33}$ & $\mathrm{U}_{23}$ & $U_{13}$ & $U_{12}$ \\
\hline $\mathrm{S} 1$ & $0.0266(4)$ & $0.0295(5)$ & $0.0349(6)$ & $-0.0009(4)$ & $-0.0015(5)$ & $0.0012(4)$ \\
\hline $\mathrm{C} 1$ & $0.032(2)$ & $0.022(2)$ & $0.030(2)$ & $-0.001(2)$ & $0.006(2)$ & $-0.005(2)$ \\
\hline $\mathrm{C} 2$ & $0.030(2)$ & $0.022(2)$ & $0.027(2)$ & $0.003(2)$ & $-0.004(2)$ & $0.002(2)$ \\
\hline C3 & $0.032(2)$ & $0.025(2)$ & $0.023(2)$ & $-0.001(2)$ & $0.000(2)$ & $0.002(2)$ \\
\hline $\mathrm{C} 4$ & $0.033(2)$ & $0.023(2)$ & $0.031(2)$ & $-0.004(2)$ & $-0.004(2)$ & $0.003(2)$ \\
\hline C5 & $0.033(2)$ & $0.031(2)$ & $0.034(2)$ & $-0.007(2)$ & $-0.001(2)$ & $-0.004(2)$ \\
\hline C6 & $0.039(2)$ & $0.025(2)$ & $0.025(2)$ & $-0.003(2)$ & $-0.007(2)$ & $0.003(2)$ \\
\hline $\mathrm{C7}$ & $0.058(3)$ & $0.048(3)$ & $0.024(2)$ & $-0.002(2)$ & $-0.006(2)$ & $0.000(2)$ \\
\hline C8 & $0.036(2)$ & $0.055(3)$ & $0.039(3)$ & $-0.009(2)$ & $-0.009(2)$ & $-0.008(2)$ \\
\hline $\mathrm{Cg}$ & $0.027(2)$ & $0.029(2)$ & $0.020(2)$ & $0.001(2)$ & $-0.004(2)$ & $0.004(2)$ \\
\hline $\mathrm{C} 10$ & $0.037(2)$ & $0.024(2)$ & $0.026(2)$ & $0.002(2)$ & $-0.002(2)$ & $0.003(2)$ \\
\hline $\mathrm{C} 11$ & $0.029(2)$ & $0.030(2)$ & $0.028(2)$ & $-0.005(2)$ & $-0.002(2)$ & $0.005(2)$ \\
\hline $\mathrm{C} 12$ & $0.027(2)$ & $0.031(2)$ & $0.024(2)$ & $-0.005(2)$ & $-0.003(2)$ & $0.000(2)$ \\
\hline $\mathrm{C} 13$ & $0.035(2)$ & $0.025(2)$ & $0.036(2)$ & $0.002(2)$ & $0.001(2)$ & $0.001(2)$ \\
\hline $\mathrm{C} 14$ & $0.033(2)$ & $0.022(2)$ & $0.034(2)$ & $-0.005(2)$ & $-0.002(2)$ & $0.005(2)$ \\
\hline C15 & $0.039(2)$ & $0.050(3)$ & $0.042(3)$ & $0.011(2)$ & $0.011(2)$ & $0.010(2)$ \\
\hline 01 & $0.046(2)$ & $0.032(2)$ & $0.036(2)$ & $-0.0107(12)$ & $-0.013(2)$ & $0.0030(13)$ \\
\hline $\mathrm{O} 2$ & $0.043(2)$ & $0.0225(13)$ & $0.042(2)$ & $-0.0039(13)$ & $0.0063(14)$ & $0.0020(12)$ \\
\hline $\mathrm{O} 3$ & $0.0384(14)$ & $0.0233(12)$ & $0.0220(14)$ & $0.0012(10)$ & $-0.0073(11)$ & $0.0027(11)$ \\
\hline $\mathrm{O} 4$ & $0.036(2)$ & $0.035(2)$ & $0.031(2)$ & $-0.0030(12)$ & $0.0078(13)$ & $0.0001(12)$ \\
\hline $\mathrm{O} 5$ & $0.0309(13)$ & $0.0330(13)$ & $0.041(2)$ & $0.0018(14)$ & $0.0059(13)$ & $0.0023(11)$ \\
\hline
\end{tabular}


Table 4. Bond Distances in Compound 2026, $\AA$

\begin{tabular}{|llllll|}
\hline S1-O4 & $1.498(3)$ & S1-C5 & $1.815(4)$ & S1-C2 & $1.867(4)$ \\
C1-O2 & $1.211(4)$ & C1-O1 & $1.325(4)$ & C1-C2 & $1.495(5)$ \\
C2-C3 & $1.531(5)$ & C3-O3 & $1.449(4)$ & C3-C9 & $1.512(5)$ \\
C3-C4 & $1.535(5)$ & $\mathrm{C} 4-\mathrm{C} 5$ & $1.531(5)$ & C6-O3 & $1.405(4)$ \\
C6-O1 & $1.477(4)$ & $\mathrm{C} 6-\mathrm{C} 7$ & $1.513(6)$ & $\mathrm{C} 6-\mathrm{C} 8$ & $1.513(6)$ \\
$\mathrm{C} 9-\mathrm{C} 10$ & $1.395(5)$ & $\mathrm{C} 9-\mathrm{C} 14$ & $1.402(5)$ & $\mathrm{C} 10-\mathrm{C} 11$ & $1.384(5)$ \\
$\mathrm{C} 11-\mathrm{C} 12$ & $1.384(5)$ & $\mathrm{C} 12-\mathrm{C} 13$ & $1.380(5)$ & $\mathrm{C} 12-\mathrm{O} 5$ & $1.381(4)$ \\
$\mathrm{C} 13-\mathrm{C} 14$ & $1.388(5)$ & $\mathrm{C} 15-\mathrm{O} 5$ & $1.434(5)$ & & \\
\hline
\end{tabular}

Table 5. Bond Angles in Compound 2026, ${ }^{\circ}$

\begin{tabular}{|llllll|}
\hline O4-S1-C5 & $106.1(2)$ & O4-S1-C2 & $107.8(2)$ & C5-S1-C2 & $92.1(2)$ \\
O2-C1-O1 & $119.7(4)$ & O2-C1-C2 & $122.1(3)$ & O1-C1-C2 & $118.2(3)$ \\
C1-C2-C3 & $115.0(3)$ & C1-C2-S1 & $106.2(3)$ & C3-C2-S1 & $108.6(2)$ \\
O3-C3-C9 & $113.1(3)$ & O3-C3-C2 & $106.2(3)$ & C9-C3-C2 & $116.8(3)$ \\
O3-C3-C4 & $103.7(3)$ & C9-C3-C4 & $111.6(3)$ & C2-C3-C4 & $104.3(3)$ \\
C5-C4-C3 & $107.4(3)$ & C4-C5-S1 & $107.7(3)$ & O3-C6-O1 & $112.6(3)$ \\
O3-C6-C7 & $106.0(3)$ & O1-C6-C7 & $105.6(3)$ & O3-C6-C8 & $113.4(3)$ \\
O1-C6-C8 & $105.2(3)$ & C7-C6-C8 & $113.9(4)$ & C10-C9-C14 & $116.8(3)$ \\
C10-C9-C3 & $119.8(3)$ & C14-C9-C3 & $123.4(3)$ & C11-C10-C9 & $122.2(3)$ \\
C12-C11-C10 & $119.4(3)$ & C13-C12-O5 & $115.4(3)$ & C13-C12-C11 & $120.4(3)$ \\
O5-C12-C11 & $124.2(3)$ & C12-C13-C14 & $119.7(3)$ & C13-C14-C9 & $121.6(3)$ \\
C1-O1-C6 & $124.2(3)$ & C6-O3-C3 & $119.4(3)$ & C12-O5-C15 & $117.8(3)$ \\
\hline
\end{tabular}

